\title{
Semiconductor Nanocrystals Based on Group IV Materials: Synthesis and Characterization towards Applications in Solar Cells
}

by

Angélique Faramus

\begin{abstract}
A thesis
Submitted to the Victoria University of Wellington In fulfilment of the requirements for the degree of Doctor of Philosophy in Chemistry
\end{abstract}

Victoria University of Wellington 



\section{Abstract}

Quantum dots have attracted a lot of interest in the past decade due to their physical and chemical properties. Quantum dots offer exciting possibilities for third generation photovoltaic devices. Light emitting quantum dots are stronger emitters than conventional organic dyes and are more resistant to degradation.

This thesis focuses on the solution phase synthesis of semiconducting nanoparticles containing only easily available and relatively non-toxic materials, unlike cadmium containing nanoparticles. As an example, CdSe has been heavily studied for its outstanding optical properties. But the toxicity of cadmium encourage towards the use of other materials combining low toxicity with efficient emitting properties, such as silicon or germanium. We concentrate our research to silicon, germanium, tin, tin/germanium and $\mathrm{Cu}_{2} \mathrm{ZnSnS}_{4}$ (CZTS) nanoparticles. Tin based nanocrystals are poor emitters but have great potential as light harvesters in solar cells due to great semiconducting properties. The potential applications, crystal structures and properties of the target materials are described in Chapter 1. Chapter 2 details the characterization techniques used to define the nanoparticles synthesized in this research. Size and shape of the nanocrystals was evaluated using Transmission Electron Microscopy (TEM). The crystals structure was determined by X-ray diffraction (XRD) or Selected Area Electron Diffraction (SAED). The surface termination of quantum dots was assessed via Fourier Transform Infrared Spectroscopy (FTIR). Finally, the optical properties were determined using UV-Visible and photoluminescence spectroscopies.

Silicon quantum dots (SiQDs) exhibit strong blue photoluminescence. The emission phenomenon of silicon nanostructures is still heavily debated in the literature. Chapter 3 looks into the origin of this fluorescence. The quantum dots were synthesized following a chemical reduction method in the presence of a surfactant. We evaluate the influence of the nanoparticle size variation on the optical properties. Then we explore the role of the passivation molecule on the surface of the silicon quantum dots on the light absorption and emission phenomena. 
The synthesis of CZTS nanoparticles via a solution phase process is described in Chapter 4. The aim of this research was the production of small monodisperse particles. We investigate the influence of the solvent environment in high temperature decomposition syntheses, followed by the study of a novel chemical reduction method for CZTS nanocrystals

Chapter 5 regroups the research conducted on germanium and tin quantum dots, as well as the study on germanium/tin alloy. Germanium quantum dots, strong light emitters, were characterized optically in this study. The semiconducting phase of tin has great physical properties but is unstable in an ambient environment. So far reported tin nanoparticles synthesized via a solution process display only the metallic structure of tin. Presenting similar structural properties, germanium is expected to stabilize the quantum dot configuration when alloyed to tin.

In Chapter 6 are described three different collaborative projects towards the application of silicon quantum dots in solar cells. First silicon quantum dots were anchored to zinc oxide nanowires arrays. Then we investigated the optical properties of SiQDs blended in a matrix of block copolymers. The third project looks into the effect of SiQDs spread over the surface of a working silicon solar cell.

Finally, the last chapter presents an overall conclusion and summarizes the main findings of this study. It also introduces perspectives for future work with concepts on how to overcome the problems encountered in this research and ideas towards concrete industrial application of quantum dots. 


\section{Acknowledgements}

During the course of my PhD, I have acquired lots of knowledge about science as well as personal skills enrichment. Over those three years, I had the great pleasure to meet many talented people. I would like to thank in particular:

My supervisor Richard Tilley for his wisdom and guidance throughout my PhD journey. His vast knowledge about science led to great advice and discussions. I am also thankful for his support through difficult times, both personal and professional.

My secondary supervisor Natalie Plank for her help, encouragement and for introducing me to the equipment of the cleanroom.

Amane Shiohara for introducing me to the world of silicon quantum dots and her great company and exuberance and Sujay Prabakar for his help and great humour.

The Tilley group, past and present, especially Xuan Hao Chan, Alec Lagrow, Leah Graham, Moritz Banholzer, Benjamin McVey, Siobhan Bradley, Lucy Gloag and Wai Ruu Siah. Extra special thanks to Anna Henning for proof reading and comments throughout the writing process.

David Flynn for his help with the electron microscopes, his great company and sense of humour.

The Cleanroom team, especially Hannah Zheng, Dayna Kivell and Cameron Wood.

Professor Jim Watkins for welcoming me at the University of Massachusetts in Amherst, USA and his team, particularly Xinyu Wang, Yin Ling, Eric Anderson, Nick Hendricks and David Mesguich for their help and for the great fun playing softball, and Jo-Ann Bourguignon. Also I would like to thank Boqian Yang for his help with the PL lifetime measurements. 
Professor Maan Alkaisi of the University of Canterbury in Christchurch for his kindness and collaboration and Senthuran Sivasubramaniam for his help in the cleanroom and the fun measuring the efficiency of solar cells with sunglasses during the cold winter.

Professor Werner Paulus to be a great mentor through my undergrad and masters studies and to encourage me to follow a PhD program.

All the staff at the School of Chemical and Physical Sciences at Victoria University

All my flatmates from 8 Landcross, especially Anna, Stacey, Alex, Haruna, Denise and Monique to be such great friends and encouraging me over the course of my PhD.

Master of Arms Vicci Lamb and the Wellington Swords club for those sessions of stress releasing fencing training sessions.

My two best friends Anne-So and Julien for their unconditional support and for the long fun discussions over Skype.

My family back in France and in particular my parents for their love, understanding and support.

Finally I would like to thank FRST scholarship for funding. 


\section{Abbreviations}

$\mathrm{C}_{12} \mathrm{E}_{5}=$ Pentaethylene glycol monododecyl ether

e- = electron

EDS = Energy Dispersive X-ray Spectroscopy

$\mathrm{eV}=$ electron volt

$\mathrm{FF}=$ Fill Factor

FTIR = Fourier Transformed Infra-Red

FWHM = Full width at half maxima

HRTEM = High Resolution Transmission Electron Microscopy

JCPDS = Joint Committee on Powder Diffraction Standards

LED = Light Emitting Diode

$M-J=$ multi-junction

NMR = Nuclear Magnetic Resonance

$\mathrm{NW}=$ NanoWire

ODE = Octadecene

OLA = Oleylamine

$\mathrm{PL}=$ Photoluminescence

PLE $=$ Photoluminescence excitation

QDs = Quantum Dots

QDSSC = Quantum dots-sensitized solar cell

SAED $=$ Selected Area Electron Diffraction

TEM = Transmission Electron Microscope / Microscopy

TOAB = Tetraoctyl ammonium bromide

UV-Vis = Ultra-Violet-Visible

$\mathrm{XRD}=\mathrm{X}$-Ray Diffraction 


\section{Table of Contents}

\section{Chapter 1: Introduction}

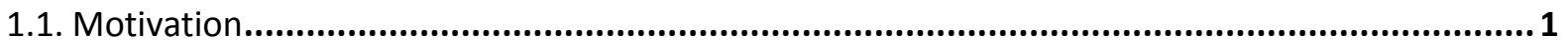

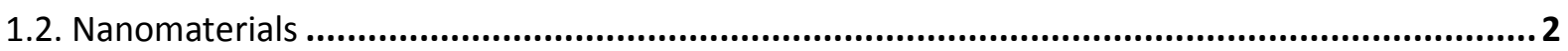

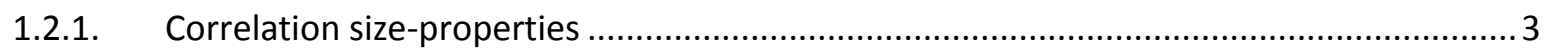

1.2.2. Energy bands and quantum confinement .................................................................. 4

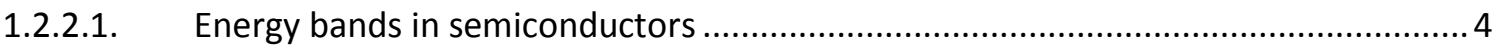

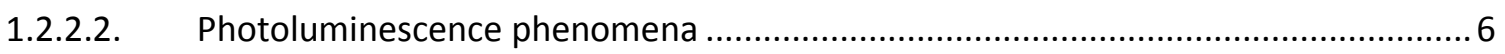

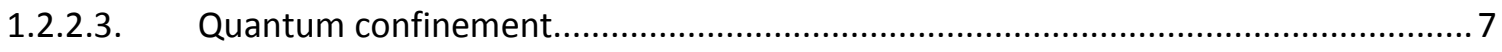

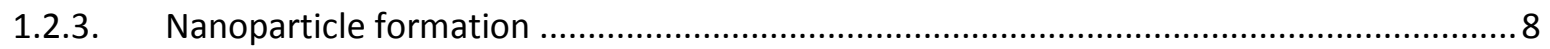

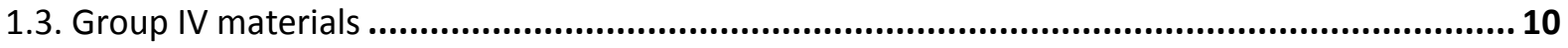

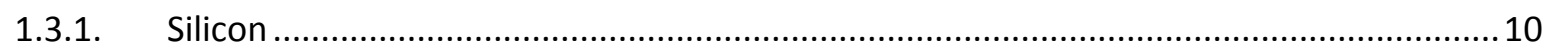

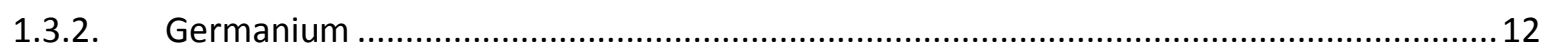

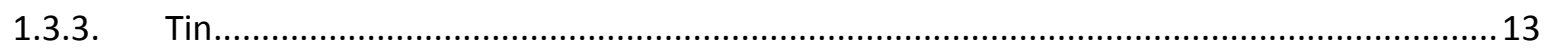

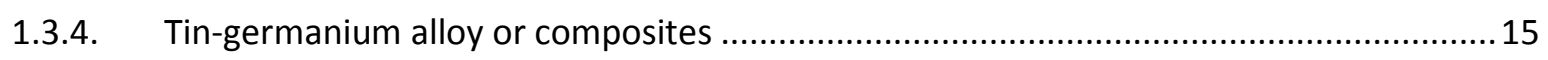

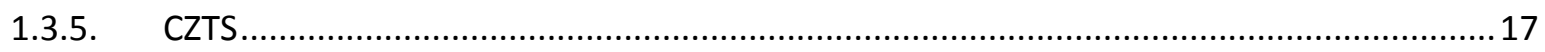

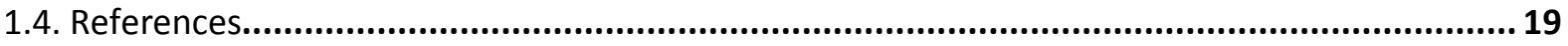

\section{Chapter 2: Experimental techniques}

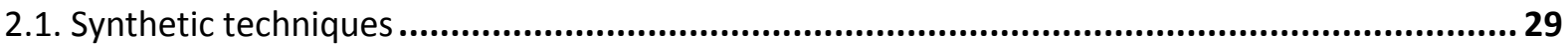

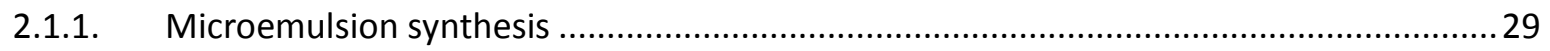

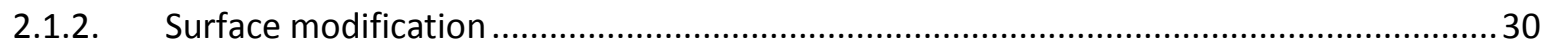

2.1.3. High temperature decomposition and hot injection synthesis .......................................30

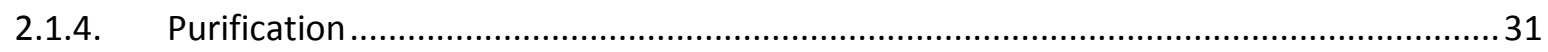

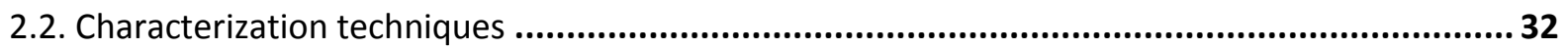

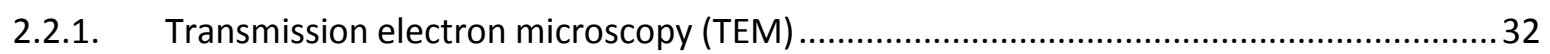

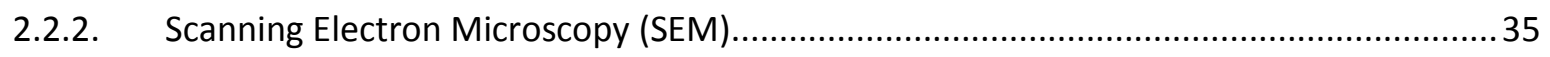

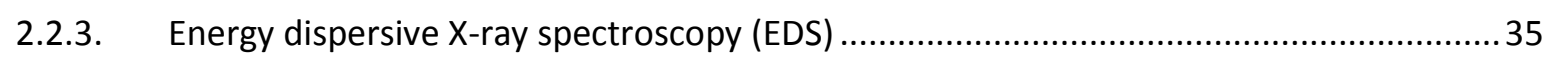

2.2.4. Diffraction techniques: $\mathrm{X}$-ray powder diffraction (XRD) and selected area electron

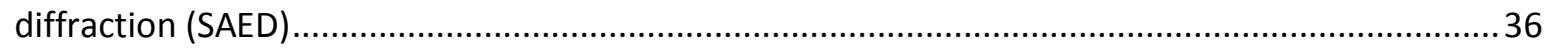

2.2.5. Fourier Transform Infrared Spectroscopy....................................................................... 38

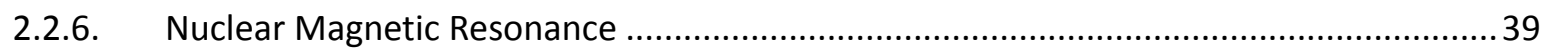

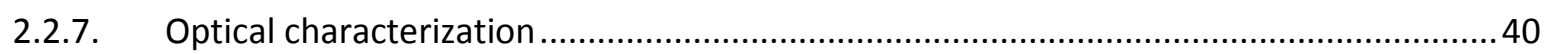

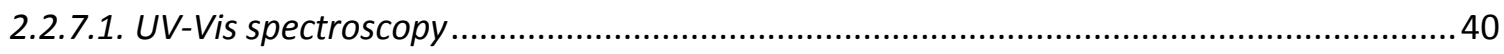


2.2.7.3. Time-resolved photoluminescence spectroscopy ......................................................... 41

\section{Chapter 3: Origin of the photoluminescence in silicon quantum dots}

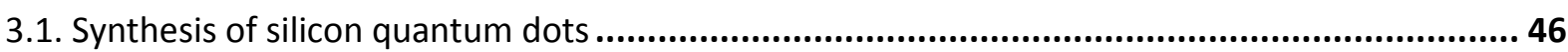

3.1.1. Hydride terminated silicon quantum dots synthesis ....................................................46

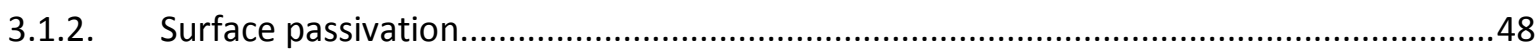

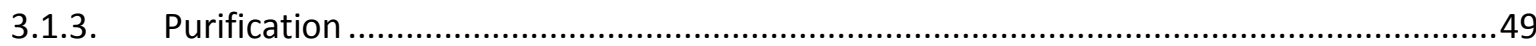

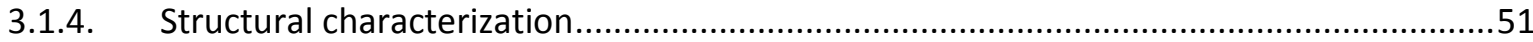

3.2. Optical properties of silicon quantum dots and their origin ....................................................55 55

3.3. Particle size effect on the optical properties of silicon quantum dots ......................................61 61

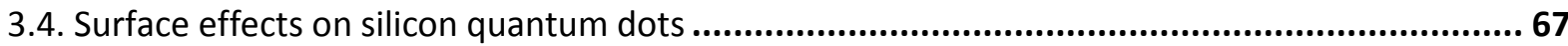

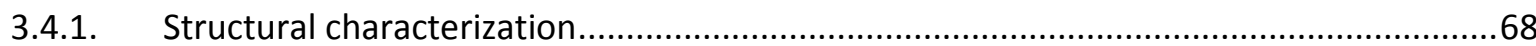

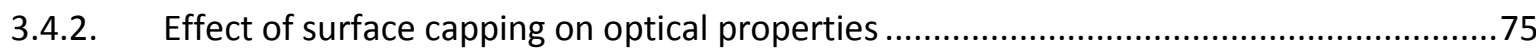

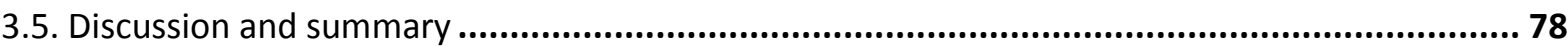

3.5.1. Origin of the photoluminescence of silicon quantum dots..............................................

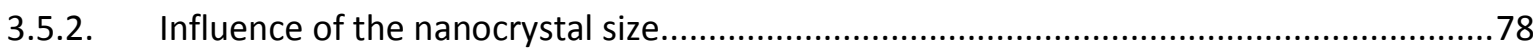

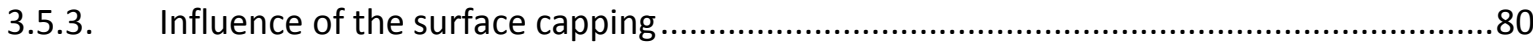

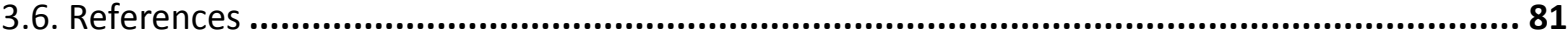

Chapter 4: CZTS nanocrystals synthesis and characterization

4.1. High temperature synthesis and influence of solvent environment ........................................ 89

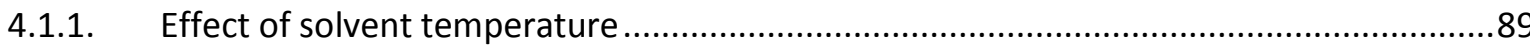

4.1.2. Effect of solvent coordination on CZTS nanocrystals formation...................................95

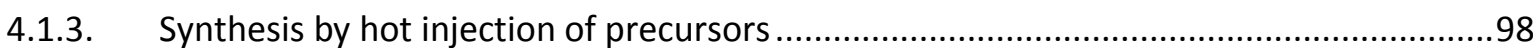

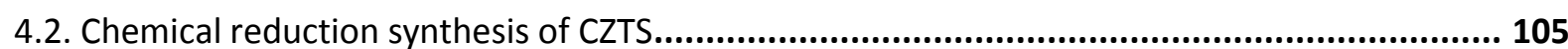

4.2.1. Experiments 7 to 11: Use of a reducing agent prior to high temperature treatment ....105

4.2.2. Room temperature synthesis by chemical reduction based on the reverse micelle principle 116

4.3. Microemulsion synthesis of metal sulphides

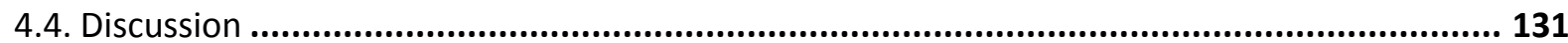

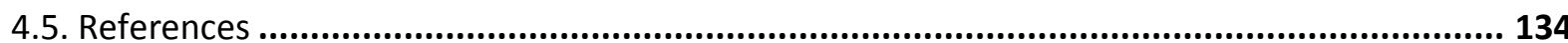

Chapter 5: Germanium, tin and tin/germanium alloy nanoparticles

5.1. Germanium quantum dots synthesis and optical properties .......................................... 142

5.2. Synthesis of tin nanoparticles via chemical reduction ....................................................... 151 


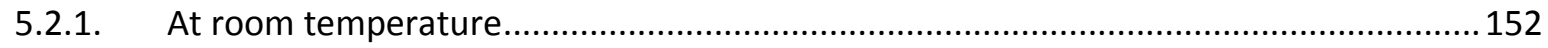

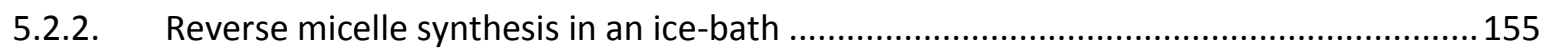

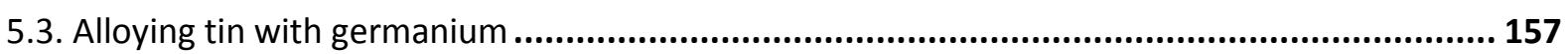

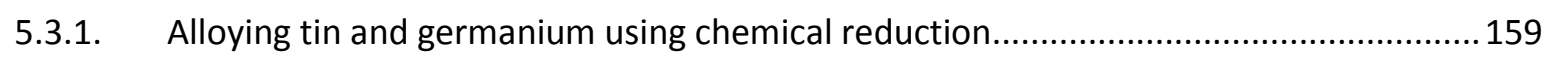

5.3.2. Alloying tin with germanium using temperature decomposition....................................161

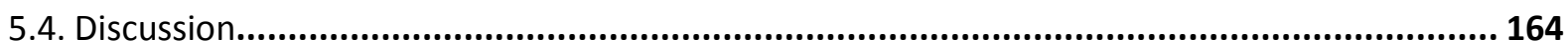

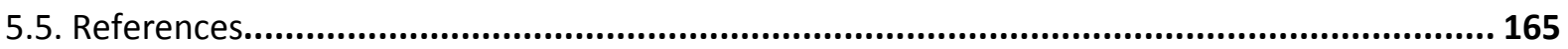

Chapter 6: Silicon quantum dots: towards their use in solar cells

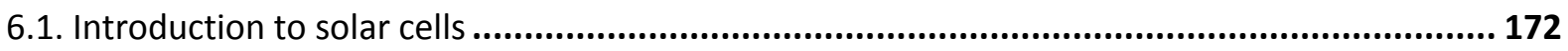

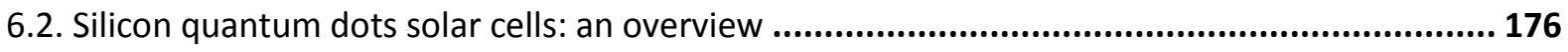

6.3. Silicon quantum dots anchored to zinc oxide nanowires .................................................... 177

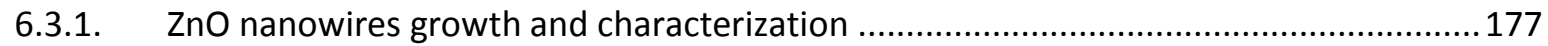

6.3.2. Silicon quantum dots and zinc oxide nanowires composite ........................................180

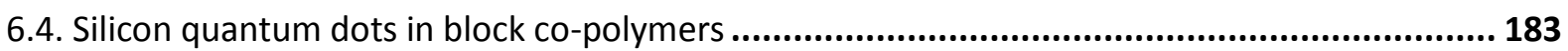

6.5. Silicon quantum dots coated on silicon solar cell............................................................. 188

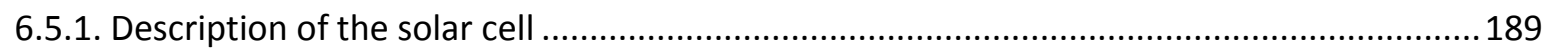

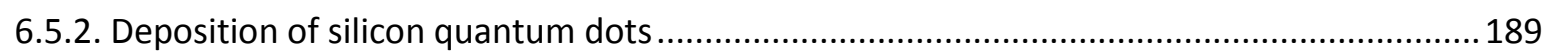

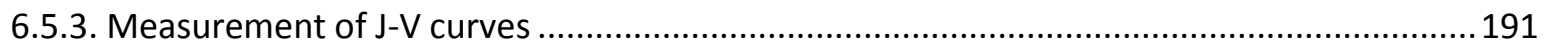

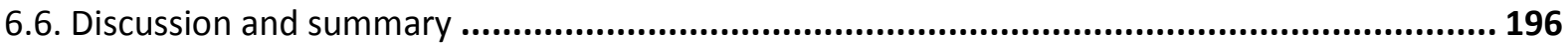

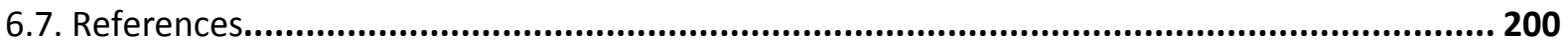

\section{Chapter 7: Conclusion}

7.1. Micro-emulsion synthesis and effect of reducing agent ........................................................209

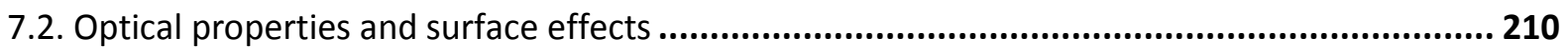

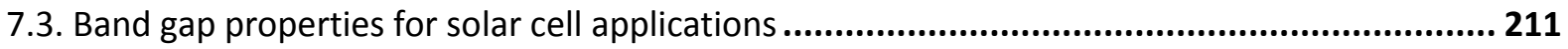

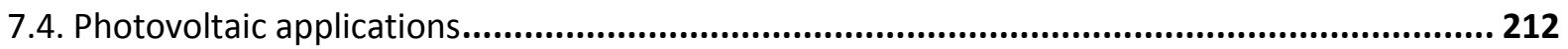

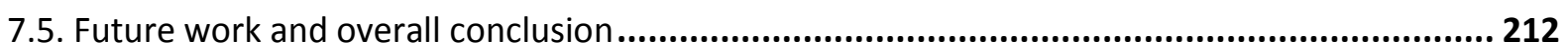

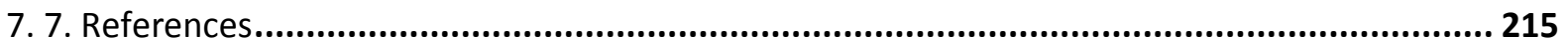

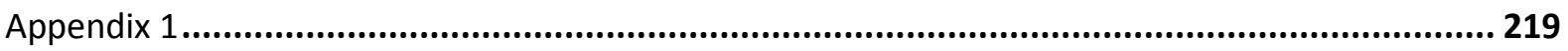

List of Publications: 


\section{Introduction}

\subsection{Motivation}

A majority of the world's current electricity supply is generated from fossil fuels such as coal, oil and natural gas. The resources in fossil fuels are finite and are decreasing rapidly, leading to rising prices, among other disagreements. Their extraction and usage raises concerns about the decline of the environment due to a dangerous level of greenhouse gas emission, and the possible cause of concerning climate changes. Renewable energy sources such as solar, biomass, geothermal, hydroelectric and wind power generation have emerged as potential alternatives.

The solar energy represents 89 petaWatts over the surface of the Earth absorbed by sea water and land. Photovoltaic energy has the most potential to address the predicted fossil fuel shortage.

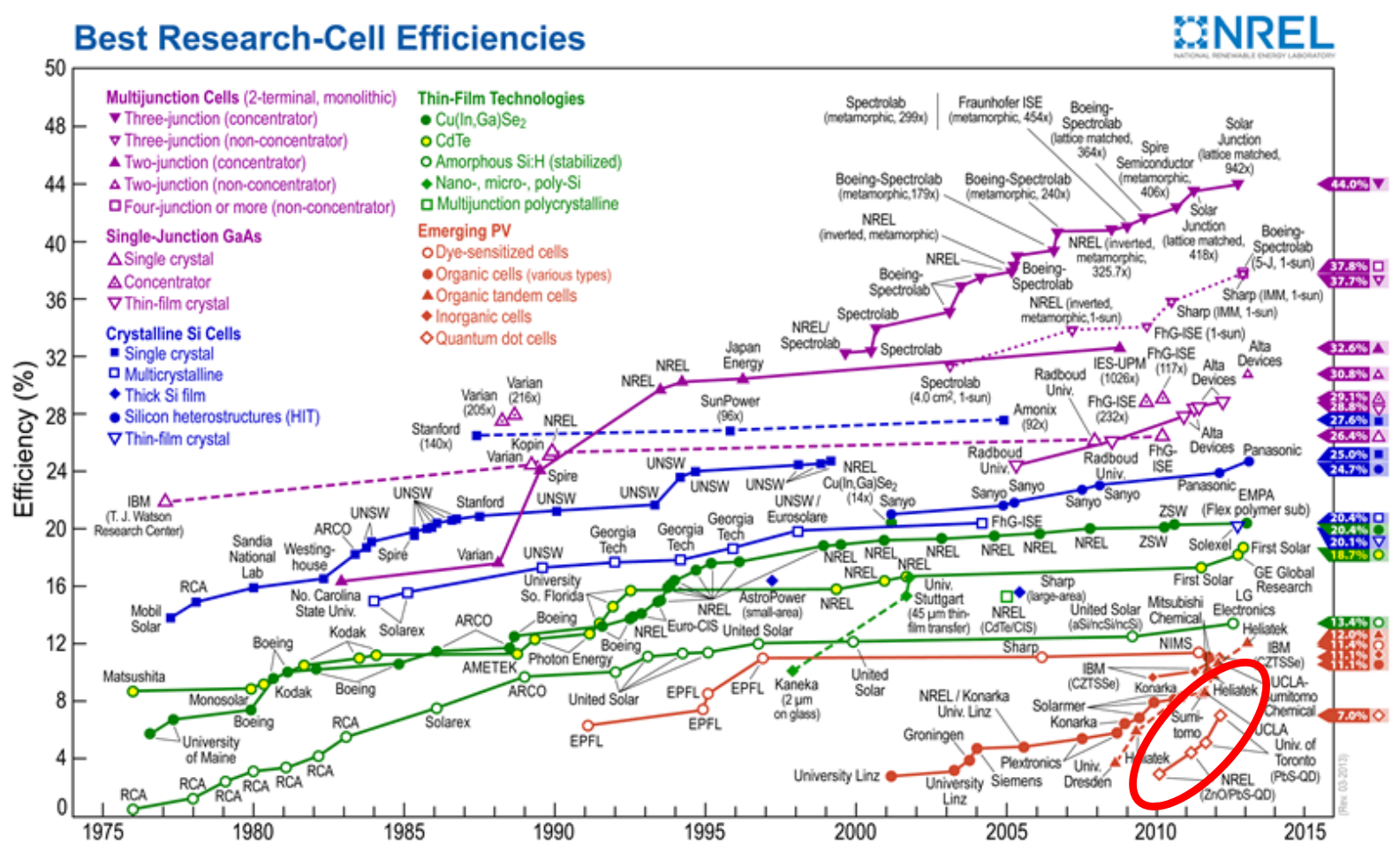

Figure 1.1: Solar cell efficiencies chart, with circled in red the quantum dots cells. ${ }^{1}$ 
Many photovoltaic devices architectures and principles are researched today. The National Renewable Energy laboratory (US) reports every year a chart of the best research-cell efficiencies (figure 1.1). ${ }^{1}$ Even though in the NREL chart it is noticeable that quantum dots solar cells are far from the efficiencies recorded for multi-junction cells, they present a high potential for the next generation of photovoltaic devices. ${ }^{2}$

Cadmium based quantum dots are by far the most studied semiconducting nanoparticles. Their outstanding efficiency and their great tunability attracted lots of interest for optoelectronic applications, including LEDs and solar cells. Unfortunately, cadmium is a highly toxic element and has been classified as a potentially dangerous element for humans and for the environment. ${ }^{3,4}$ It has been banned from electronic applications in Europe and soon to be in North America. ${ }^{5}$

Over the past decade, attention has been given to other semiconducting nanoparticles with a low toxicity. In this project, the research was focused on four different materials, based on elements from group IV: silicon, germanium, CZTS and finally tin and tingermanium alloys, and their potential syntheses, properties and applications in photovoltaics.

\subsection{Nanomaterials}

Nanomaterials are by definition a material with at least one of three dimensions comprised between 1 and $100 \mathrm{~nm}$. One or two nanosized dimension leads to nanoplate or nanotube structures. In the case of nanoparticles, the three dimensions are below $100 \mathrm{~nm}$. On a size scale, the maximum size of a nanomaterial is a thousand times smaller than the diameter of a hair. ${ }^{7}$ Nanocrystals refer to nanosized single crystalline nanoparticles, while nanoparticle is a more generic term for any kind of particle, amorphous, polymeric or crystalline.

Nanoparticles have attracted a great deal of attention over the past two decades, due to their unusual properties and characteristics compared to their bulk material counterparts. Unusual electrical, magnetic or optical properties can often be observed in the nanoparticles. ${ }^{8-10}$ Those properties can be explained by two main causes: the surface area and quantum effects. Below $100 \mathrm{~nm}$, the quantum effects are indeed annexing 
some other bulk effects, leading to new material behaviours and also the surface area to volume ratio becomes much larger when the size is decreased down to the nanoscale. Nanocrystals are the ideal candidates for many different applications, such as gassensors, electronic devices, biological markers, solar cells for a widespread large production. ${ }^{11}$ Taking in consideration a low cost of production as well as the environmentally friendly synthesis becomes an increasing factor for the use of nanocrystals.

\subsubsection{Correlation size-properties}

The main difference between bulk and nanosized materials is the ratio between surface area and volume. In nanoparticles, a larger fraction of the atoms are indeed located on the surface. Figure 1.2.a represents the size dependence of the number of atoms located either in the core or at the surface of gold nanoparticles. In many biological applications, such as drug delivery, the increased surface area per volume ratio permits a larger number of functional group attached to the surface per volume of material, so a better efficiency.

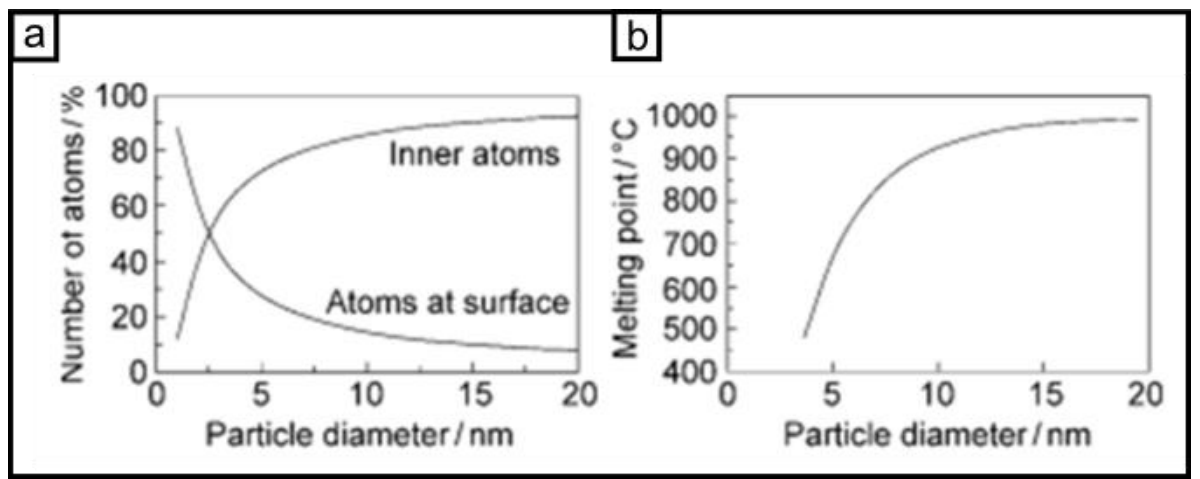

Figure 1.2: a) Number of surface and inner atoms dependence as a function of gold particle diameter and b) relationship between particle size and melting temperature for gold particles. ${ }^{12}$

The decrease of particle size also leads to a change of intrinsic properties. Buffat et al has reported a decrease of the melting temperature with gold particles (fig. 1.2.b). ${ }^{12}$ Similar behavior has been observed for other metal nanoparticles such as lead or tin. ${ }^{13,14}$ Magnetic material can become superparamagnetic under a critical size. 
Superparamagnetic particles have no residual magnetization after exposure to a magnetic field, so have no risk to aggregate. ${ }^{15}$ These nanocrystals can be used in medical applications as MRI contrast agents. Another property of particles in the nanoscale is the ability to absorb and emit in different regions of the light, due to surface effects. Gold or silver nanocrystals are well-known for exhibiting different colours depending on their size range due to a phenomenon called surface plasmon resonance. The discovery of this property might have been the first exploited property of the nanoparticles, as it has been found that gold nanoparticles are present in coloured glass objects dating from Ancient Roman times. But the largest number of possible applications today is probably for semiconductor nanocrystals. Below a certain size, they enter a state called quantum confinement which can give rise to a new range of properties such as photoluminescence.

\subsubsection{Energy bands and quantum confinement}

\subsubsection{Energy bands in semiconductors}

Materials can be separated into three main categories depending on their electrical conductivity: metals, semiconductors and insulators. The difference is determined by their atomic energy levels.

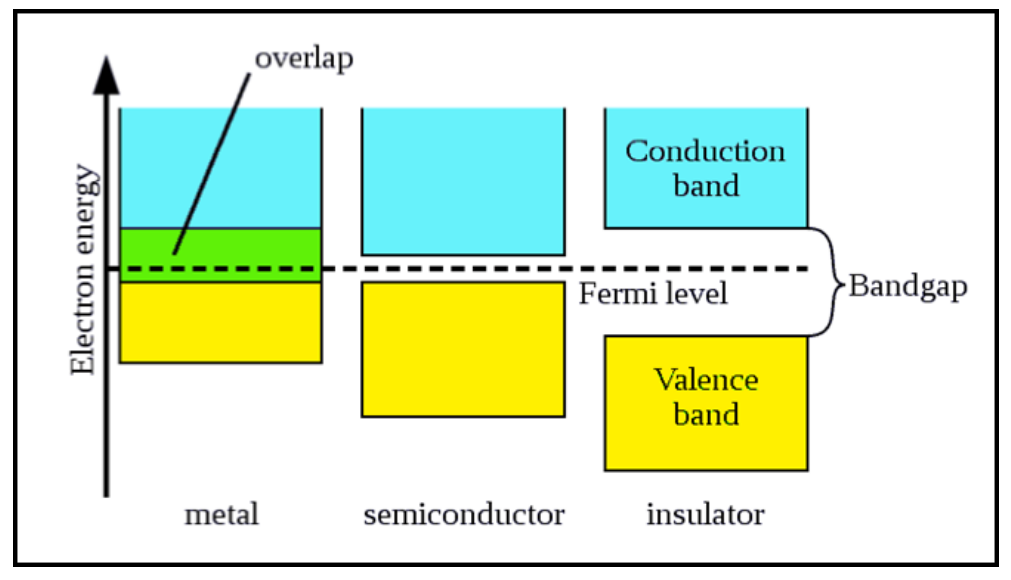

Figure 1.3: Energy level difference between metals, semiconductors and insulators. ${ }^{16}$

All materials present two sets of energy levels, confined in bands called valence band and conduction band. The Valence band, which is lower in energy, is considered as the 
stable or low energy state. It contains most of the electrons of the material. On the contrary, the conduction band is almost empty. The energy levels in the conduction bands are also called excited states. For metals, the two sets of energy levels overlap, so the electrons move freely upon energy excitation and without resistance, so the electrical conductivity is optimum. The electrons in an insulator are stable and confined in the Valence band. In semiconductors, the top of the valence band, also called HOMO (Highest Occupied Molecular Orbital) and the bottom of the conduction band, LUMO (Lowest Unoccupied Molecular Orbital), are separated by a gap in energy of 'forbidden states' called the band gap $\left(E_{g}\right)$. Through applied electrical bias, light or heat, enough energy can be given to electrons to move from Valence to conduction bands.

The energy of electrons varies upon their position in an atom. Due to the conservation of momentum principle, energy band structures are not constant and vary with the momentum $\mathrm{p}$ of electrons. The wavevector $\mathrm{k}$ represents the momentum equivalent and the relative position of an electron in the reciprocal space. ${ }^{17}$ Due to the energy band variation, two types of energy gap transitions can be observed in semiconductors: direct or indirect. Figure 1.4 represents the band structure of GaAs, a direct band gap semiconductor and silicon, which has an indirect band gap. A semiconductor presents a direct band gap when the maximum energy of the Valence band $(E V)$ and the minimum energy value of the conduction band $(E c)$ are presenting the same wavevector $k=0$. This transition can be initiated by the absorption of a photon. On the contrary, when the minimum of the conduction band is situated at different values of the wavevector $k$, and since it does not coincide to the maximum of the Valence band, the band gap is called indirect. In the case of an indirect bandgap, there is a very low probability to observe the emission of light, as the emission requires the simultaneous production of a phonon and a photon. 


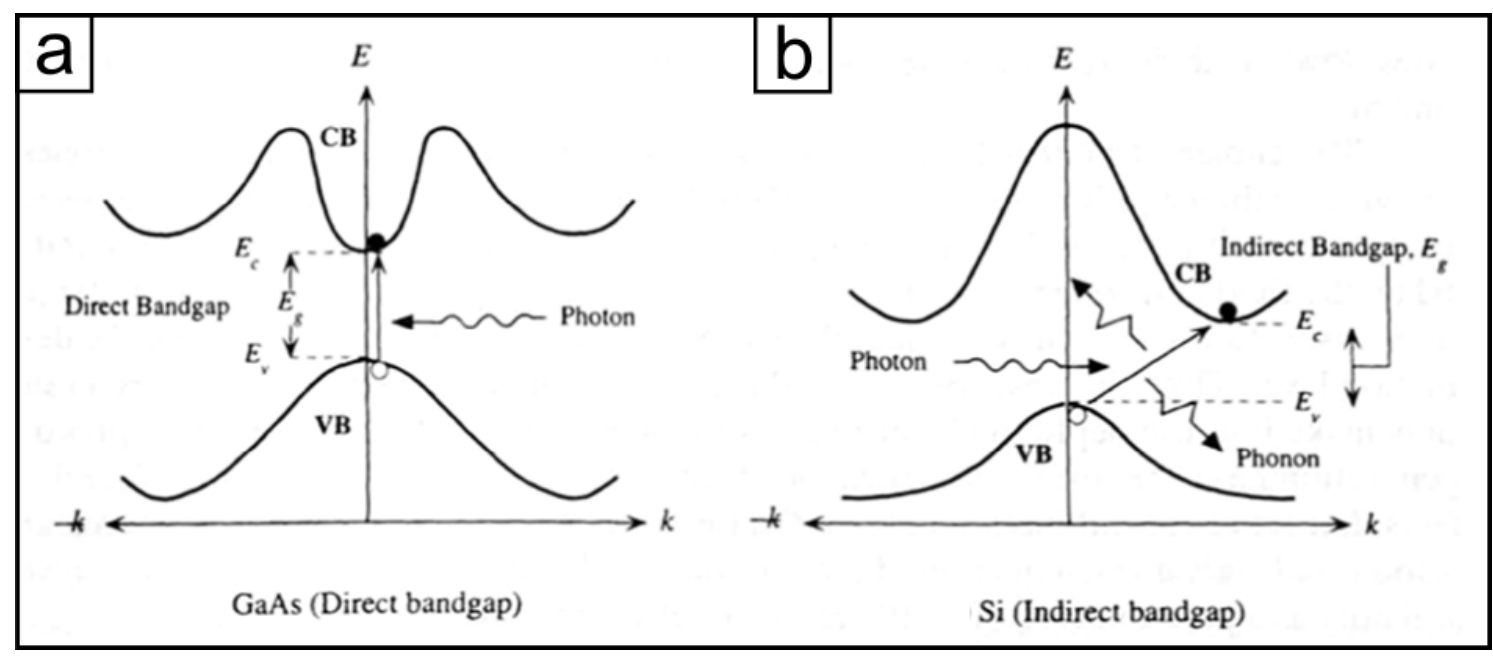

Figure 1.4: Band gap photon absorption process for a) a direct band gap semiconductor, GaAs and b) an indirect band gap semiconductor, silicon. ${ }^{18}$

\subsubsection{Photoluminescence phenomena}

Semiconductors get their name from the property of the electrons to go from the valence band to the conduction band if they receive an energy greater than the band gap. If a direct band gap semiconductor is exposed to light radiation, with a photon energy (hv) higher than the energy of the gap, an electron can be excited to the conduction band, creating a hole in the Valence band. The longest wavelength for the excitation of an electron corresponds to the absorption spectraledge of the semiconductor. The electron-hole pair thus generated is called an exciton. This configuration is unstable, so the electron will have the tendency to recombine with the hole. When the electron returns to its stable configuration in the Valence band, it releases the energy by re-emitting a photon, which energy (hv) is characteristic of the band gap. This radiation can be light in the visible light region of the electromagnetic wave spectrum, so the material emits light with a colour characteristic of the radiation wavelength, and this phenomenon is called photoluminescence. For semiconductors with an indirect band gap, the recombination process must be mediated by a phonon. $\mathrm{A}$ photon and a phonon must be associated to create a radiative recombination of an exciton in an indirect band gap semiconductor. As the probability of photon-phonon combination is low, the photoluminescence is not observed for indirect band gap materials. $^{18}$ 


\subsubsection{Quantum confinement}

The exciton Bohr radius is the spatial distance between the hole and the electron in an exciton. When at least one of the dimensions of nanocrystals approaches the bulk exciton Bohr radius, nanoparticles enters the quantum confinement regime and those semiconducting nanocrystals are called quantum dots. ${ }^{19}$

On the nanoscale, semiconductor energy levels of the valence and conduction band become discrete. ${ }^{20}$ The band gap of a semiconductor also increases with decreasing particle size owing to statistically reduced positions for electrons. Due to the increase of the bandgap while reducing the size of the nanocrystals, the wavelengths of the absorption edge and of the photoluminescence change result in a blue spectral shift. Many studies have reported the blue shift in the PL or the absorption for different quantum dots, such as CdSe and InP. ${ }^{21,22}$

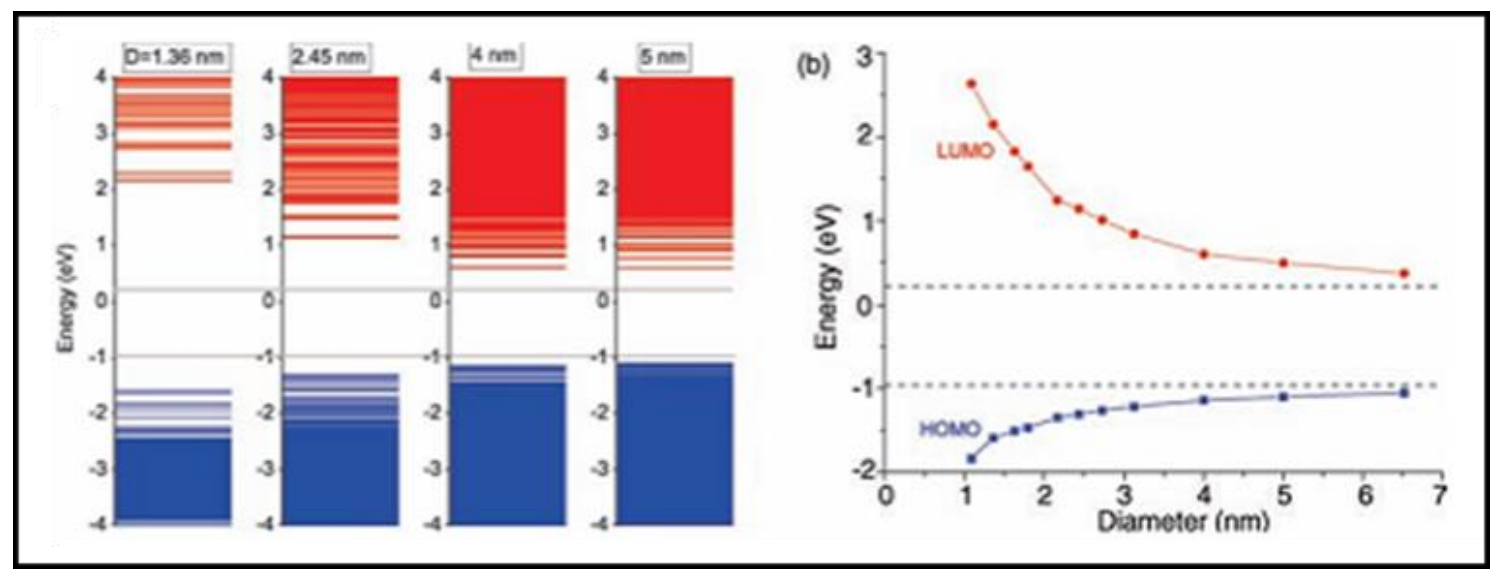

Figure 1.5: Evolution of energy bands of silicon nanocrystals with respect to diameter.

The dashed lines correspond to the bulk band edges. ${ }^{23}$

Group II-VI quantum dots, such as CdSe present a direct band gap, both in the bulk state and nanoparticle form. The origin of the photoluminescence is relatively easy to explain. For group IV materials, such as silicon and germanium, the photoluminescence of the quantum dots is more complex. Both $\mathrm{Si}$ and $\mathrm{Ge}$ are indirect band gap semiconductors. But when their size is below the exciton Bohr radius, their energy levels are confined in a small environment and a direct energy transition between the Valence band and the 
conduction band becomes possible, explaining the strong luminescence observed for silicon and germanium quantum dots. ${ }^{24,25}$

\subsubsection{Nanoparticle formation}

Two different ways of preparing nanoparticles have been developed, called "top-down" and "bottom-up". The "top-down" approach links to physical synthesis methods, where the nanostructures are obtained starting from the bulk materials and essentially by carving out the nanoparticles. Those methods include for example etching, laser ablation, lithography or sonication. ${ }^{26-28}$ Regarding the nanoparticle size, the "top-down" approach is more appropriate to the bigger nanoparticles (> 50nm diameter). Achieving smaller particles would require more complex equipment and it would not be a favourable choice for large scale commercial production.

The "bottom-up" approach refers to chemical synthesis methods. In the "bottom-up" approach, the synthesis starts from species at the atomic scale and particles grow until reaching a nanometric size. "Bottom-up" types of synthesis deal with either gas or liquid phase approaches. Molecular beam epitaxy (MBE), chemical vapour deposition (CVD) and solution phase synthesis are examples of "bottom-up" synthesis. Vapour phase synthesis offers a good control over the structure and composition of the product, but requires high vacuum atmospheres and high temperatures, highly energy consuming. Solution phase synthesis allows a great particle size control without the requirement of expensive equipment or high temperature. Furthermore, reagents are relatively cheap and no dangerous solid by-products are produced during most syntheses make solution phase synthesis the strongest candidate for later stage mass production.

As most of the properties of the nanoparticles are size-dependant, their monodispersity is a very important factor. Monodispersion can be described as a regularity of size, composition and shape among the nano-sized objects and is usually considered to be inferior to $10 \%$ of particle size distribution. ${ }^{29}$

A solution phase synthesis requires the use of a precursor and a surfactant. A precursor, usually a metal salt or a complex containing the desired ions, is either decomposed by 
temperature or reduced by a reducing agent and atoms become monomers that aggregate to form particles. Surfactants can bind to the surface of the nanoparticles, limiting their growth and controlling their final size and shape. ${ }^{30}$

The mechanism of formation of nanoparticles is commonly described in the literature using the LaMer model, which gives a theoretical explanation to the production of monodisperse sulphur hydrosols. ${ }^{31}$

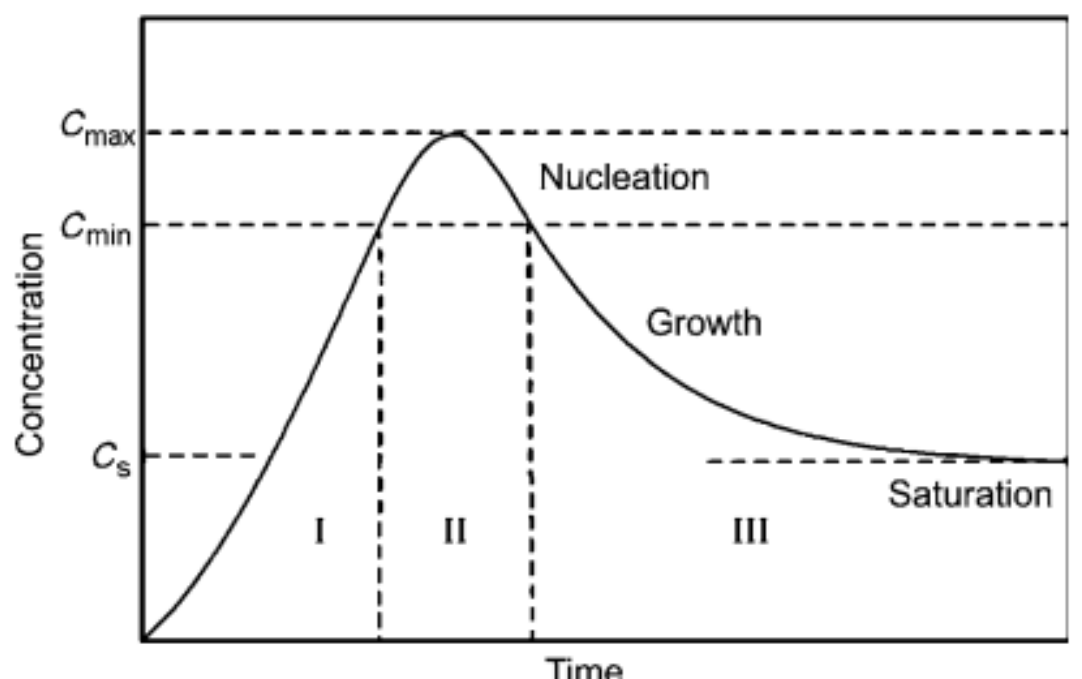

Figure 1.6: LaMer diagram representing the three stages (I, II and III) of nanoparticles growth depending on the concentration of monomers as a function of time. ${ }^{31}$

The bottom-up model is composed of three phases relating to the nucleation and growth of nanoparticles. In stage I, as the decomposition progresses, the concentration of monomers increases. The production of monomers stops when the concentration reaches the super-saturation state, which corresponds to the maximum concentration of monomers $\left(C_{\max }\right)$. At this point (phase II), the monomers start to form stable nuclei, and so the concentration of those monomers decreases. $C_{\min }$ corresponds to the minimum concentration of monomers to form nucleis. During the stage III, the growth process takes place by diffusion of the remaining monomers in solution onto the surface of the nuclei and thus forming nanoparticles. In order to obtain monodisperse nanoparticles, the duration of the nucleation phase has to be decreased to a single event, called 'burst nucleation'. The simultaneous nucleation insure of the uniformity in growth of the nanoparticles, leading to monodisperse nanocrystals in solution. 


\subsection{Group IV materials}

\subsubsection{Silicon}

Silicon is the second most abundant element in the Earth's crust. It is widely used in electronic technology for its low cost and electronic properties. In the bulk form, silicon has an indirect band gap of $1.12 \mathrm{eV}(1100 \mathrm{~nm})$. Due to this indirect bandgap, bulk silicon exhibits almost no photoluminescence at any wavelength. But under quantum confinement, the indirect band gap energy levels change and silicon has been found to exhibit the properties approaching that of a pseudo-direct band gap material. The discovery of this quantum effect on silicon is relatively recent, as it has been reported for the first time in 1991 by Canham who discovered visible photoluminescence emission of porous silicon. ${ }^{32}$ Since then, a considerable number of studies have reported the synthesis of silicon nanostructures, from nanoparticles to nano film depositions. The methods used have involved either a physical or a chemical approach. Most of the possible applications in electronic devices would require that silicon quantum dots display tuneable fluorescence and absorption wavelengths. Many different photoluminescence wavelengths have been reported in the literature. ${ }^{32-37}$ The PL properties are strongly dependent to the size of the nanocrystals, suggesting a directtype energy transition in the quantum confinement size region. The surface capping of the silicon dots are also playing an important role in the emission of photoluminescence. ${ }^{38-41}$ Most physical methods reported thin films with a thin oxide layer on the surface, or silicon nanoparticles in a $\mathrm{SiO}_{2}$ matrix. The $\mathrm{Si}-\mathrm{O}$ bond on the surface of oxidized quantum dots leads to wider spectrum emission, with photoluminescence up to $700 \mathrm{~nm}$. The origin of this fluorescence is more challenging to explain than the direct band gap transition. The principal theory invokes defects at the interface between the $\mathrm{Si}$ core and the $\mathrm{SiO}_{2}$ shell. ${ }^{42-44}$ Using the solution phase reduction synthesis, the surface can be modified to produce either hydrophobic to hydrophilic surface suing various organic molecules. ${ }^{45}$ Those organic molecules play a role in the photoluminescence phenomenon. But the strong blue to green luminescence combined with a surface hydrophobic or hydrophilic property makes silicon quantum dots suitable for diverse utilization, from optical to biomedical applications. ${ }^{46,47}$ 
Several silicon quantum dot synthetic methods are already reported in the literature. As an example of physical synthesis, Kortshagen produces SiQDs via a gas-phase plasma approach. ${ }^{48}$ These employing the solution phase approach, Veinot's, Tilley's, Kauzlarich's and Korgel's are the leading groups. Veinot and co-workers reported the thermal decomposition of hydrogen silsesquioxane (HSQ) producing oxide embedded silicon nanocrystals that are acid etched using hydrofluoric acid. ${ }^{49,50}$ Before using Veinot's method, Korgel developed a synthesis method using supercritical solvents. ${ }^{51-53}$ Kauzlarich reported silicon quantum dots synthesized via the decomposition of $\mathrm{NaSi}$ salts. $^{54,55}$ In 2005 was first reported the sized controlled synthesis of SiQDs via the microemulsion process by the Tilley group. ${ }^{56}$

Silicon crystallises in a diamond-cubic type structure. The lattice parameter at ambient conditions of temperature and pressure has a dimension of $0.543 \mathrm{~nm}$. The energy level structure represented in figure 1.7.b shows the indirect band gap nature of bulk silicon. ${ }^{57,58}$

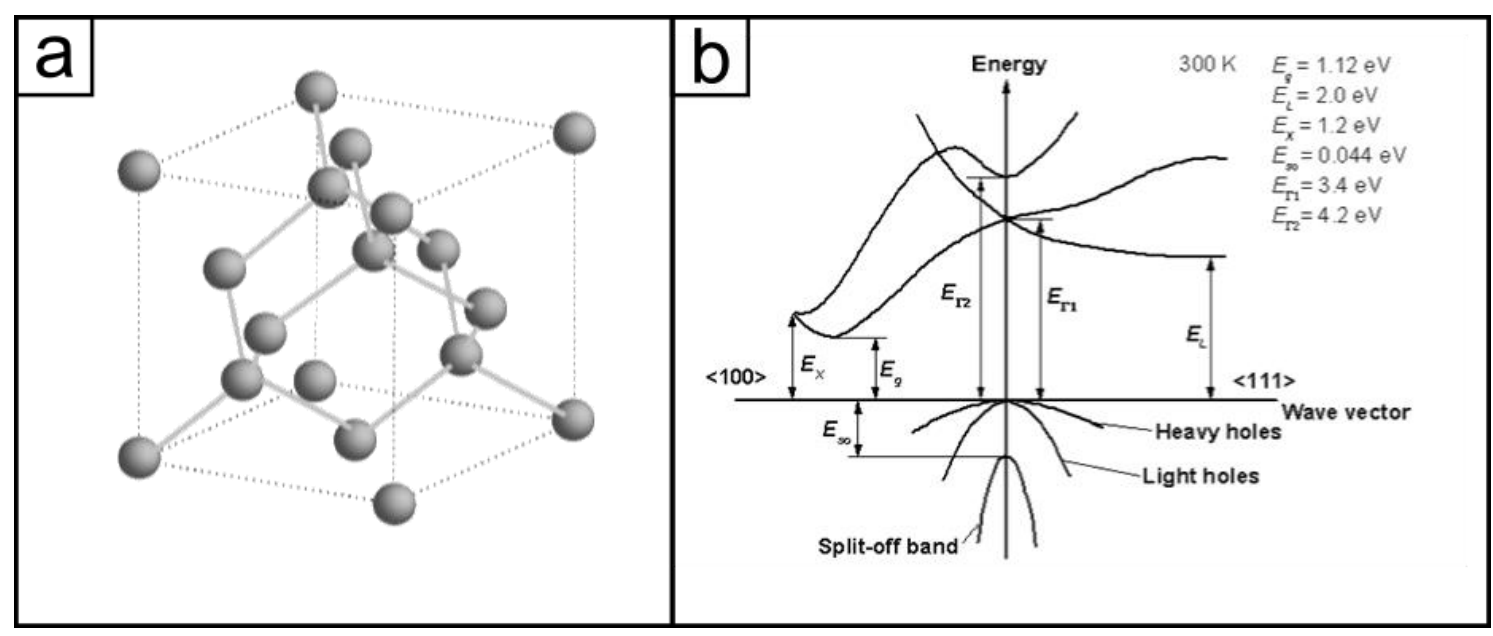

Figure 1.7: a) Silicon diamond-cubic crystal structure and b) silicon energy levels in function of wavevector at $300 \mathrm{~K} .^{57,58}$

The different possible energy transitions between the Valence band and the conduction band in silicon crystals are represented in figure 1.7.b. The energy values listed correspond to energy transitions between atomic positions in the reciprocal space. The variation in wavevector between the maximum energy value of the Valence band and 
the minimum energy of the conduction band confirms the indirect nature of the band gap of silicon.

\subsubsection{Germanium}

Germanium is the third element of the group IV and has many features similar to silicon. Ge also crystallises in the diamond cubic structure and presents an indirect band gap of $0.66 \mathrm{eV}(1880 \mathrm{~nm})$ at $300 \mathrm{~K}$ in the bulk form. Figure 1.8 represents the energy band structure as a function of wavevector $k$. The energy values listed correspond to energy transitions between atomic positions in the reciprocal space. In a similar way than silicon (fig. 1.7.b), the variation in wavevector between the maximum energy value of the Valence band and the minimum energy of the conduction band confirms the indirect nature of the band gap of germanium. By decreasing the size of the crystal below the excitonic Bohr radius of $11.5 \mathrm{~nm}$, germanium exhibits direct band gap material behaviour, thus imparting a strong photoluminescence in the visible region. ${ }^{59}$ The Bohr radius value of germanium is much larger than for silicon, allowing the germanium to maintain the fluorescent properties at much larger sizes.

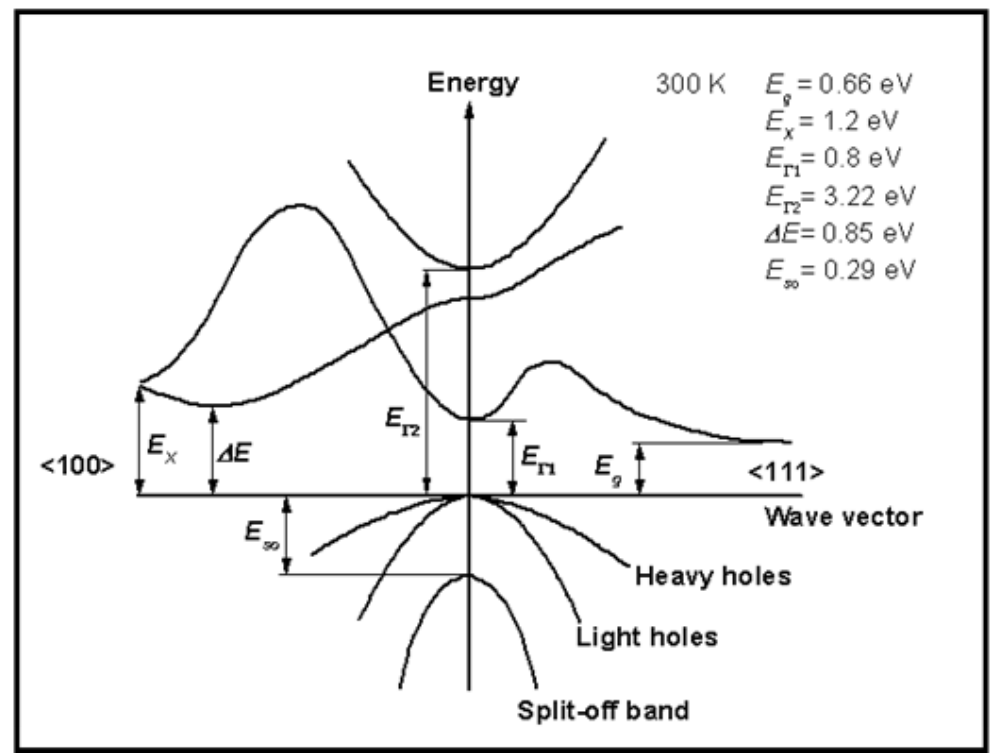

Figure 1.8: Wavevector dependence of the band structure of germanium at $300 \mathrm{~K}^{60}$

Germanium nanocrystals have been less intensively studied than silicon, mostly due to the better resistance to temperature and lower price of silicon. However, several synthetic methods for germanium nanocrystals have been reported, including solution phase synthesis. 
Regarding the physical synthesis methods, Kortshagen and co-workers reported a nonthermal plasma dissociation of germanium tetrachloride producing Ge nanoparticles in the 4-50 nm size range. ${ }^{61}$ Stoldt et al reported the preparation of 3 to $14 \mathrm{~nm}$ sized freestanding germanium nanocrystals by ultrasonic aerosol pyrolysis. ${ }^{62}$ The solution phase synthesis methods are the most reported for the formation of germanium nanoparticles. Kauzlarich et al reported a solution method using combination of $\mathrm{NaGe}$ with $\mathrm{GeCl}_{4}$ or $\mathrm{NH}_{4} \mathrm{Br}^{63,64}$ Also, butyl-capped crystalline $\mathrm{Ge}$ nanoparticles were synthesized at room temperature in dimethoxyethane by reduction of $\mathrm{GeCl}_{4}$ with sodium naphthalide and subsequent reaction with butyl Grignard reagent, followed by annealing at $550-600^{\circ} \mathrm{C}$ to obtain crystalline particles. ${ }^{65} \mathrm{Lu}$ and co-workers formed germanium nanocrystals by thermolysis of germanium precursors in supercritical solvents. ${ }^{66}$ An ultrasonic solution reduction method based on the reduction of $\mathrm{GeCl}_{4}$ by metal hydride or alkaline in ambient condition has been reported by Wu et al. ${ }^{67}$ The nanocrystals with a $5 \mathrm{~nm}$ size exhibit a $352 \mathrm{~nm}$ photoluminescence emission. Wilcoxon and co-workers described an inverse micelle synthesis by reduction of an ionic salt by lithium aluminium hydride. ${ }^{59} \mathrm{~A} \mathrm{PL}$ in the range $350-700 \mathrm{~nm}$ was observed from nanocrystals $2-5 \mathrm{~nm}$ in size. Tilley et al reported the reduction of $\mathrm{GeCl}_{4}$ using a strong reducing agent produces Ge quantum dots with a narrow size distribution. ${ }^{68,69}$ The surface is modified by allylamine to render the nanoparticles hydrophilic. 420 to $480 \mathrm{~nm}$ photoluminescence emission peaks are observed.

The influence of the surface chemistry and the particle size of the germanium nanocrystals synthesized by solution phase synthesis on the optical properties needs to be investigated. The visible photoluminescence wavelength exhibited by small monodispersed particles makes germanium quantum dots suitable for applications in opto-electronic devices.

\subsubsection{Tin}

Tin is the fourth element in the group IV or 14 of the periodic table. Tin is a well-known metal for having a low toxicity. In its bulk form, tin is still used as coating for food packaging, in electrical components or ornamental objects. The ordinary form of the tin 
is called white tin or $\beta$-tin. It exhibits a tetragonal crystal structure. Compared to noble metals, metallic tin presents a relatively low melting temperature of $232^{\circ} \mathrm{C}^{70}$

Below $13^{\circ} \mathrm{C}, \beta$-tin transforms into a diamond cubic-type structure, also called grey-tin or $\alpha$-tin. ${ }^{71}$ This transformation between $\beta$ and $\alpha$ tin provokes a deformation at a macroscopic scale of metallic tin objects, called "tin pest". The cubic arrangement of $\alpha$ tin presents the properties of a semi-conductor, with a bandgap of $0.08 \mathrm{eV}$.

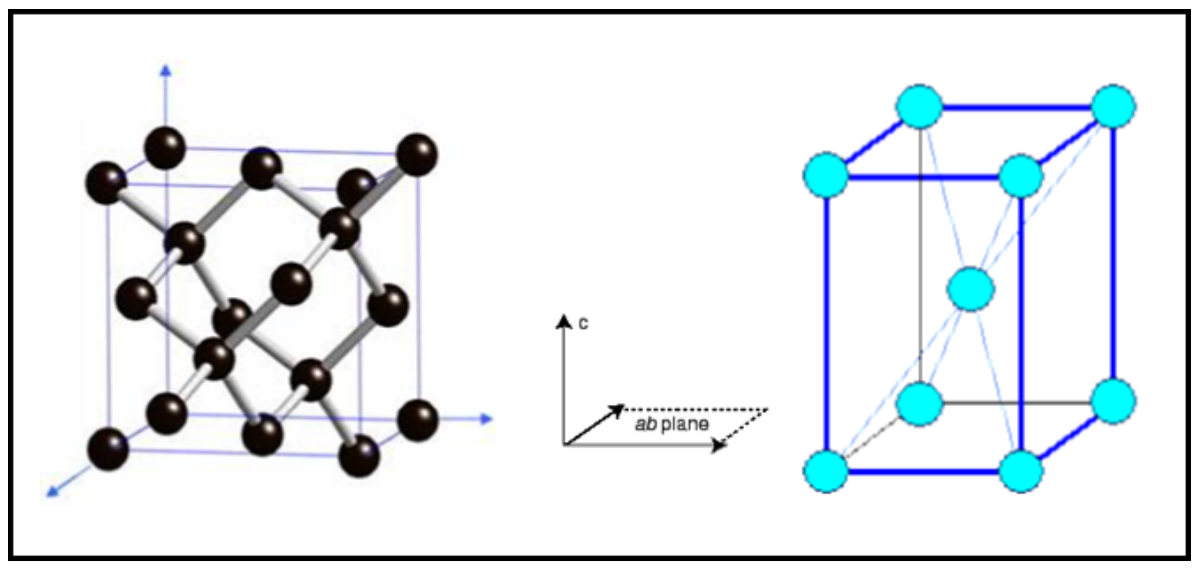

Figure 1.9: On the left, the $\alpha$-Sn cubic structure, with lattice parameters being $a=6.341$

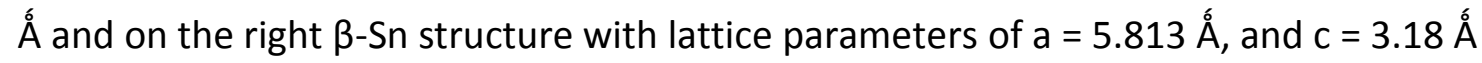

$\beta$-tin nanoparticles have been intensively studied. Li et al prepared tin nanoparticles from the bulk state via ultrasonic dispersion. ${ }^{72}$ The nanoparticles ranged from 50 to $3500 \mathrm{~nm}$ decreasing size with ultrasound power. Lai et al reported a thermal evaporation process and Zou et al synthesized $\beta$-tin nanocrystals by chemical reduction using sodium borohydride. ${ }^{73,74}$ All those studies describe synthetic methods for tetragonal phase of tin and a surface oxidation. No surface treatment has been conducted on the nanoparticles so they spontaneously form a shell of $\mathrm{SnO} / \mathrm{SnO}_{2}$ by exposure to air. Huang et al reported the fabrication of $\beta$ phased tin-based nanocrystals in a matrix by using a co-sputtering technique. ${ }^{75}$ They found that the nanocrystals have the propensity to oxidize in a $\mathrm{SiO}_{2}$ matrix, while in a nitride matrix, which is a reducing environment, tin nanocrystals are not subject to oxidation. ${ }^{76}$ Nanosized $\beta$-tin crystals also present a melting temperature depression by decreasing average particle size. White tin nanocrystals can be used to replace noble metals, such as gold or silver, for many applications as low melting temperature metallic nanoparticles. Jo et al. reported 
a conductive ink application of small and monodisperse Sn nanocrystals obtained by reduction of tin acetate. ${ }^{77}$ The conductivity measurements are of comparable order of magnitude to noble metals ( $\mathrm{Au}, \mathrm{Ag}$ ). Cui et al synthesized nanorods by chemical reduction in presence of a dodecyl sulphate surfactant and they observed the melting temperature decrease with nanostructure size and described their application as nanosoldering materials. ${ }^{78}$

Concerning the grey-cubic phase of tin, no solution-phase synthesis of pure $\alpha$-tin has been reported yet. This phase has already been obtained by physical methods. Wang et al reported the cubic phase of tin confined in a nanotube of tin oxide, $\mathrm{SnO}_{2} \cdot{ }^{79}$ This structure has been achieved by molecular beam epitaxy. They observed an increase of the stability temperature of the diamond cubic phased tin from $13.2^{\circ} \mathrm{C}$ in the bulk form to up to $700^{\circ} \mathrm{C}$. The stabilization of $\alpha$-tin has also been reported by Asom et al in the thin film form, deposited onto InSb substrates by molecular beam epitaxy. ${ }^{80}$

The tin nanoparticles presenting the diamond cubic structure are expected to behave as a semiconductor. As the bulk $\alpha$-tin have a band gap of $0.08 \mathrm{eV}$, an infrared photoluminescence emission would be expected. For smaller nanoparticles, if the nanoparticles enter the quantum confinement regime, there would be an expected increase in the bandgap and consequently a blue shift of the PL emission.

\subsubsection{Tin-germanium alloy or composites}

A possible way to stabilize the grey tin structure at temperatures higher than $13.2^{\circ} \mathrm{C}$, the transition temperature for conversion into white tin at ambient pressure, would be to alloy tin with another material with diamond cubic structure. Silicon and Germanium spontaneously crystallise in the diamond cubic form and some examples of such an alloy with tin have been reported in the literature. ${ }^{83-84}$ Potentially, tin/germanium alloy can lead to an interesting semiconductor. ${ }^{81}$

The Sn-Ge solid solution presents a very low solubility of germanium into tin. King et al stated a maximum concentration of $1 \%$ of $\mathrm{Ge}$ into $\mathrm{Sn} .^{82}$ This very low solubility is probably due to the large lattice mismatch between the two elements, which are 5.646 
$\AA$ for Ge and $6.489 \AA$ for $\alpha$-tin and to the fact that at ambient conditions of temperature and pressure, the cubic-phase of tin is unstable.

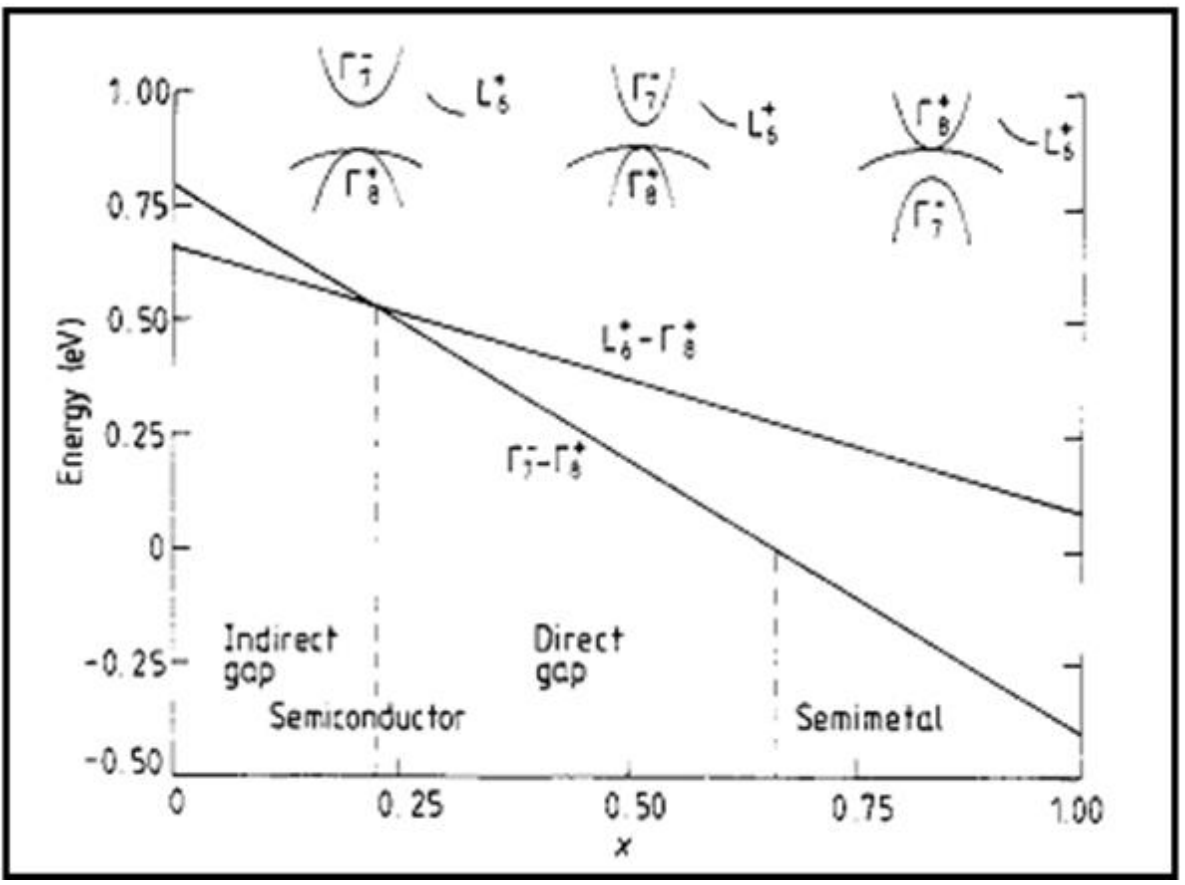

Figure 1.10: Variation of energy levels disposition in $\mathrm{Ge}_{1-\mathrm{x}} \mathrm{Sn}_{\mathrm{x}}$ alloys. $^{81}$

Interestingly, the character of the band gap changes with the concentration of tin in the solid solution $\mathrm{Ge}_{1-\mathrm{x}} \mathrm{Sn}_{\mathrm{x}}$. At very low concentrations $(x<0.25)$, the band gap transition is indirect, as the one of bulk germanium, but with increasing tin concentration, the band gap becomes direct. ${ }^{81}$ At higher concentrations ( $x>0.70$ ), GeSn alloys exhibit the properties of a semimetal. The greek letters $\Gamma$ and $L$ represent atomic positions in the reciprocal space and their associated energy transitions.

Pukite et al reported a single phase $\mathrm{Sn}_{\mathrm{x}} \mathrm{Ge}_{1-\mathrm{x}}$ grown by molecular beam epitaxy, with $\mathrm{x}$ up to $0.3 .{ }^{83}$ They observed that above this concentration of $30 \%$ of tin, the film causes phase separation into the tetragonal $\beta$-tin phase. Kouvetakis and co-workers developed the growth of $\mathrm{Ge}_{1-x} \mathrm{Sn}_{\mathrm{x}}$ alloy, with $5 \%<\mathrm{x}<25 \%$, by chemical vapour deposition. ${ }^{84}$ They reported a high thermal stability of the cubic structure up to $600^{\circ} \mathrm{C}$. The diffusion of germanium in tin has also been used for the growth of germanium nanowires, using tin nanoparticles as seeds. It seems that no solution phase synthesis of Sn-Ge alloy with a diamond cubic structure has been reported in the literature. 


\subsubsection{CZTS}

$\mathrm{Cu}_{2} \mathrm{ZnSnS}_{4}$ or CZTS is a quaternary semiconductor composed from cheap, abundant and non-toxic materials: copper, zinc, tin and sulphur. It has received an increasing interest since the late 2000 s for its application in solar cells as a light-absorbing material. ${ }^{85}$

Quaternary semiconductor nanostructures are challenging to synthesize, because of difficulty in controlling the stoichiometry and the coalescence of the four elements together to form one unique structure.

CZTS has a direct band gap comprised between 1.4 and $1.5 \mathrm{eV}$, which is the ideal band gap for high efficiency third generation photovoltaics. ${ }^{86,87}$ CZTS also benefits from a large absorption coefficient. ${ }^{88}$

CZTS can be found in two different crystal structures. The most favourable structure is the tetragonal kesterite structure. CZTS can also be found in the stannite crystal structure. More recently, CZTS has been synthesized in a hexagonal wurtzite configuration. $^{92}$

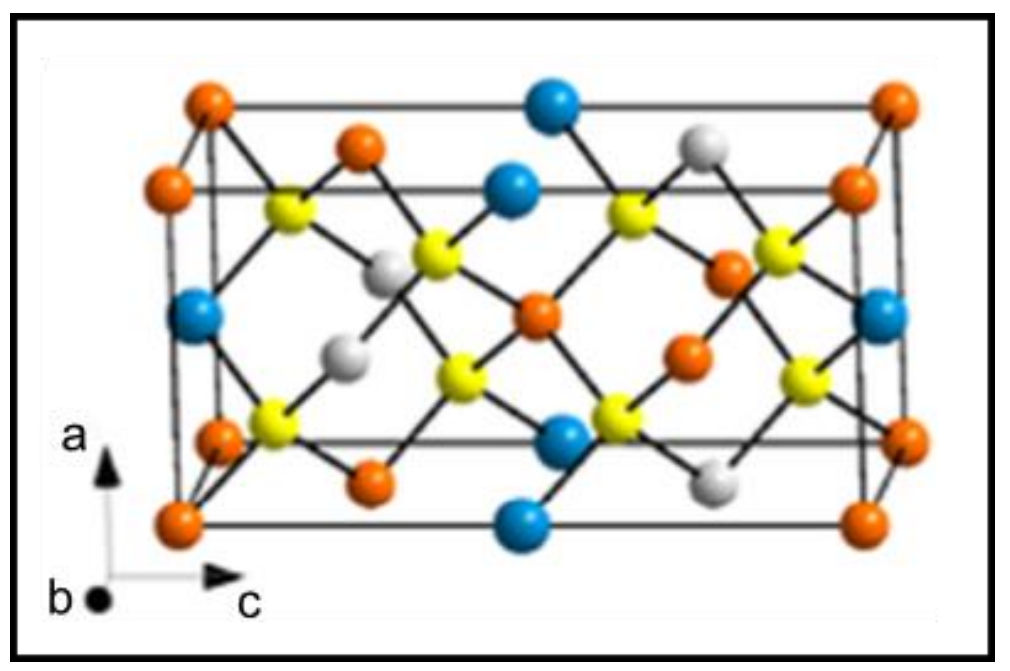

Figure 1.11: CZTS kesterite crystal structure. (Orange: $\mathrm{Cu}$, grey: $\mathrm{Zn}$, blue: $\mathrm{Sn}$, yellow: S).

CZTS are mostly reported in the form of thin films. CZTSSe (kesterite-type crystal structure) solar cells were first reported by Katagiri in 1996, with $0.66 \%$ power conversion efficiencies for the initial vacuum-deposited devices. ${ }^{89}$ Recently, a solar cell based on a CZTS thin film achieved a $11 \%$ efficiency. ${ }^{90}$ 
CZTS nanoparticles can be synthesized via a solution phase process. CZTS nanocrystals are compatible with deposition techniques such as printing and casting, each of which are well-established industrial procedures. ${ }^{91}$ Solution-phase synthetic processes have the potential for a good phase and size control. One-pot synthesis by temperature decomposition methods have been reported to be successful for both the wurtzite and kesterite phases. ${ }^{92-94}$ In this research will be investigated the effect of the solvent environment on the CZTS nanoparticles size, shape and structure in colloidal synthesis. Also, the synthesis via chemical reduction will be investigated, which is unprecedented for CZTS nanocrystals, with the aim to reach a better size control and possibly reach the quantum confinement regime, only reported by Khare et al in $2011 .^{95}$ 


\subsection{References}

1_http://www.nrel.gov/ncpv/

2_ Kamat, P. V., Quantum Dot Solar Cells. The Next Big Thing in Photovoltaics. The Journal of Physical Chemistry Letters 2013, 4 (6), 908-918.

3_Chandramohan, S.; Sathyamoorthy, R.; Sudhagar, P.; Kanjilal, D.; Kabiraj, D.; Asokan, K., Optical properties of swift ion beam irradiated CdTe thin films. Thin Solid Films 2008, 516 (16), 5508-5512.

4_ Wakeham, S.; Hawkins, G.; Henderson, G.; Carthey, N., Investigation of tin-based alternatives for cadmium in optoelectronic thin-film materials. Appl. Opt. 2008, 47 (13), C206-C213.

5_http://www.rsc.org/chemistryworld/News/2011/May/27051102.asp

6_Hartwig, A., Cadmium and Cancer. In Cadmium: From Toxicity to Essentiality, Sigel, A.; Sigel, H.; Sigel, R. K. O., Eds. Springer Netherlands: 2013; Vol. 11, pp 491-507.

7_ Goesmann, H.; Feldmann, C., Nanoparticulate Functional Materials. Angewandte Chemie International Edition 2010, 49 (8), 1362-1395.

8_ Badr, Y.; Mahmoud, M. A., Size-dependent spectroscopic, optical, and electrical properties of PbSe nanoparticles. Crystal Research and Technology 2006, 41 (7), 658663.

9_Park, T.-J.; Papaefthymiou, G. C.; Viescas, A. J.; Moodenbaugh, A. R.; Wong, S. S., SizeDependent Magnetic Properties of Single-Crystalline Multiferroic BiFeO3 Nanoparticles. Nano Letters 2007, 7 (3), 766-772.

10_Moreels, I.; Lambert, K.; Smeets, D.; De Muynck, D.; Nollet, T.; Martins, J. C.; Vanhaecke, F.; Vantomme, A.; Delerue, C.; Allan, G.; Hens, Z., Size-Dependent Optical Properties of Colloidal PbS Quantum Dots. ACS Nano 2009, 3 (10), 3023-3030. 
11_ Kruis, F. E.; Fissan, H.; Peled, A., Synthesis of nanoparticles in the gas phase for electronic, optical and magnetic applications--a review. Journal of Aerosol Science 1998, 29 (5-6), 511-535.

12_ Buffat, P.; Borel, J. P., Size effect on the melting temperature of gold particles. Physical Review A 1976, 13 (6), 2287.

13_Peters, K. F.; Cohen, J. B.; Chung, Y.-W., Melting of Pb nanocrystals. Physical Review B 1998, $57(21), 13430$.

14_Jiang, H.; Moon, K.-s.; Dong, H.; Hua, F.; Wong, C. P., Size-dependent melting properties of tin nanoparticles. Chemical Physics Letters 2006, 429 (4-6), 492-496.

15_Lu, A.-H.; Salabas, E. L.; Schüth, F., Magnetic Nanoparticles: Synthesis, Protection, Functionalization, and Application. Angewandte Chemie International Edition 2007, 46 (8), 1222-1244.

16_ http://www.electrical4u.com/electrical-conductivity-of-metal-semiconductor-andinsulator/

17_Stroyuk, A. L.; Kryukov, A. I.; Kuchmii, S. Y.; Pokhodenko, V. D., Quantum Size Effects in the Photonics of Semiconductor Nanoparticles. Theoretical and Experimental Chemistry 2005, 41 (2), 67-91.

18_ http://www.anandtech.com/show/3834/intels-silicon-photonics-50g-siliconphotonics-link/3

19_Zorman, B.; Ramakrishna, M. V.; Friesner, R. A., Quantum Confinement Effects in CdSe Quantum Dots. The Journal of Physical Chemistry 1995, 99 (19), 7649-7653.

20_Adams, L. L. A.; Lang, B. W.; Goldman, A. M., Observation of Discrete Energy Levels in a Quantum Confined System. Physical Review Letters 2005, 95 (14), 146804.

21_ Norris, D. J.; Bawendi, M. G., Measurement and assignment of the size-dependent optical spectrum in CdSe quantum dots. Physical Review B 1996, 53 (24), 16338. 
22_Mićić, O. I.; Cheong, H. M.; Fu, H.; Zunger, A.; Sprague, J. R.; Mascarenhas, A.; Nozik, A. J., Size-Dependent Spectroscopy of InP Quantum Dots. The Journal of Physical Chemistry B 1997, 101 (25), 4904-4912.

23_Pavesi, L.; Dal Negro, L.; Mazzoleni, C.; Franzò, G.; Priolo, F., Optical gain in silicon nanocrystals. Nature 2000, 408 (6811), 440-444.

24_Takagahara, T.; Takeda, K., Theory of the quantum confinement effect on excitons in quantum dots of indirect-gap materials. Physical Review B 1992, 46 (23), 15578.

25_Kocevski, V.; Eriksson, O.; Rusz, J., Transition between direct and indirect band gap in silicon nanocrystals. Physical Review B 2013, 87 (24), 245401.

26_Nychyporuk, T.; Lysenko, V.; Gautier, B.; Barbier, D., Silicon nanoparticle formation by short pulse electrochemical etching in the transition regime. Journal of Applied Physics 2006, 100 (10), 104307-7.

27_Umezu, I.; Nakayama, Y.; Sugimura, A., Formation of core-shell structured silicon nanoparticles during pulsed laser ablation. Journal of Applied Physics 2010, 107 (9), 094318-3.

28_Bley, R. A.; Kauzlarich, S. M.; Davis, J. E.; Lee, H. W. H., Characterization of Silicon Nanoparticles Prepared from Porous Silicon. Chemistry of Materials 1996, 8 (8), 18811888.

29_Murray, C. B.; Sun, S.; Gaschler, W.; Doyle, H.; Betley, T. A.; Kagan, C. R., Colloidal synthesis of nanocrystals and nanocrystal superlattices. IBM Journal of Research and Development 2001, 45 (1), 47-56.

30_ Dumestre, F.; Chaudret, B.; Amiens, C.; Renaud, P.; Fejes, P., Superlattices of Iron Nanocubes Synthesized from Fe[N(SiMe3)2]2. Science 2004, 303 (5659), 821-823.

31_LaMer, V. K.; Dinegar, R. H., Theory, Production and Mechanism of Formation of Monodispersed Hydrosols. Journal of the American Chemical Society 1950, 72 (11), 4847-4854. 
32_ Cullis, A. G.; Canham, L. T., Visible light emission due to quantum size effects in highly porous crystalline silicon. Nature 1991, 353 (6342), 335-338.

33_ Wilcoxon, J. P.; Samara, G. A., Tailorable, visible light emission from silicon nanocrystals. Applied Physics Letters 1999, 74 (21), 3164-3166.

34_Yang, C.-S.; Bley, R. A.; Kauzlarich, S. M.; Lee, H. W. H.; Delgado, G. R., Synthesis of Alkyl-Terminated Silicon Nanoclusters by a Solution Route. Journal of the American Chemical Society 1999, 121 (22), 5191-5195.

35_ Tilley, R. D.; Yamamoto, K., The Microemulsion Synthesis of Hydrophobic and Hydrophilic Silicon Nanocrystals. advanced materials 2006, 18 (15), 2053-2056.

36_Shirahata, N.; Furumi, S.; Sakka, Y., Micro-emulsion synthesis of blue-luminescent silicon nanoparticles stabilized with alkoxy monolayers. Journal of Crystal Growth 2009, $311(3), 634-637$.

37_Holmes, J. D.; Ziegler, K. J.; Doty, R. C.; Pell, L. E.; Johnston, K. P.; Korgel, B. A., Highly Luminescent Silicon Nanocrystals with Discrete Optical Transitions. Journal of the American Chemical Society 2001, 123 (16), 3743-3748.

38_ Shirahata, N., Colloidal Si nanocrystals: a controlled organic-inorganic interface and its implications of color-tuning and chemical design toward sophisticated architectures. Physical Chemistry Chemical Physics 2011, 13 (16), 7284-7294.

39_Rosso-Vasic, M.; Spruijt, E.; van Lagen, B.; De Cola, L.; Zuilhof, H., AlkylFunctionalized Oxide-Free Silicon Nanoparticles: Synthesis and Optical Properties. Small 2008, 4 (10), 1835-1841.

40_König, D.; Rudd, J.; Green, M. A.; Conibeer, G., Role of the interface for the electronic structure of Si quantum dots. Physical Review B 2008, 78 (3), 035339.

41_Dasog, M.; Yang, Z.; Regli, S.; Atkins, T. M.; Faramus, A.; Singh, M. P.; Muthuswamy, E.; Kauzlarich, S. M.; Tilley, R. D.; Veinot, J. G. C., Chemical Insight into the Origin of Red and Blue Photoluminescence Arising from Freestanding Silicon Nanocrystals. ACS Nano 2013, 7 (3), 2676-2685. 
42_ Di, D.; Perez-Wurfl, I.; Conibeer, G.; Green, M. A., Formation and photoluminescence of $\mathrm{Si}$ quantum dots in SiO2/Si3N4 hybrid matrix for all-Si tandem solar cells. Solar Energy Materials and Solar Cells 2010, 94 (12), 2238-2243.

43_Anoop, G.; Hartmut, W., Freestanding silicon quantum dots: origin of red and blue luminescence. Nanotechnology 2011, 22 (5), 055707.

44_Wolkin, M. V.; Jorne, J.; Fauchet, P. M.; Allan, G.; Delerue, C., Electronic States and Luminescence in Porous Silicon Quantum Dots: The Role of Oxygen. Physical Review Letters 1999, 82 (1), 197-200.

45_ Shiohara, A.; Hanada, S.; Prabakar, S.; Fujioka, K.; Lim, T. H.; Yamamoto, K.; Northcote, P. T.; Tilley, R. D., Chemical Reactions on Surface Molecules Attached to Silicon Quantum Dots. Journal of the American Chemical Society 2010, 132 (1), 248-253.

46_Erogbogbo, F.; Yong, K.-T.; Roy, I.; Xu, G.; Prasad, P. N.; Swihart, M. T., Biocompatible Luminescent Silicon Quantum Dots for Imaging of Cancer Cells. ACS Nano 2008, 2 (5), 873-878.

47_Zorn, M.; Bae, W. K.; Kwak, J.; Lee, H.; Lee, C.; Zentel, R.; Char, K., Quantum Dot-Block Copolymer Hybrids with Improved Properties and Their Application to Quantum Dot Light-Emitting Devices. ACS Nano 2009, 3 (5), 1063-1068.

48_Bapat, A.; Gatti, M.; Ding, Y.-P.; Campbell, S. A.; Kortshagen, U., A plasma process for the synthesis of cubic-shaped silicon nanocrystals for nanoelectronic devices. Journal of Physics D: Applied Physics 2007, 40 (8), 2247.

49_Hessel, C. M.; Henderson, E. J.; Veinot, J. G. C., Hydrogen Silsesquioxane: A Molecular Precursor for Nanocrystalline Si-SiO2 Composites and Freestanding HydrideSurface-Terminated Silicon Nanoparticles. Chemistry of Materials 2006, 18 (26), 61396146.

50_Veinot, J. G. C., Synthesis, surface functionalization, and properties of freestanding silicon nanocrystals. Chemical Communications 2006, 0 (40), 4160-4168. 
51_Yu, Y.; Bosoy, C. A.; Hessel, C. M.; Smilgies, D.-M.; Korgel, B. A., Silicon Nanocrystal Superlattices. ChemPhysChem 2013, 14 (1), 84-87.

52_Holmes, J. D.; Ziegler, K. J.; Doty, R. C.; Pell, L. E.; Johnston, K. P.; Korgel, B. A., Highly Luminescent Silicon Nanocrystals with Discrete Optical Transitions. Journal of the American Chemical Society 2001, 123 (16), 3743-3748.

53_ Ding, Z.; Quinn, B. M.; Haram, S. K.; Pell, L. E.; Korgel, B. A.; Bard, A. J., Electrochemistry and Electrogenerated Chemiluminescence from Silicon Nanocrystal Quantum Dots. Science 2002, 296 (5571), 1293-1297.

54_Pettigrew, K. A.; Liu, Q.; Power, P. P.; Kauzlarich, S. M., Solution Synthesis of Alkyland Alkyl/Alkoxy-Capped Silicon Nanoparticles via Oxidation of Mg2Si. Chemistry of Materials 2003, 15 (21), 4005-4011.

55_Atkins, T. M.; Thibert, A.; Larsen, D. S.; Dey, S.; Browning, N. D.; Kauzlarich, S. M., Femtosecond Ligand/Core Dynamics of Microwave-Assisted Synthesized Silicon Quantum Dots in Aqueous Solution. Journal of the American Chemical Society 2011, 133 (51), 20664-20667.

56_Tilley, R. D.; Warner, J. H.; Yamamoto, K.; Matsui, I.; Fujimori, H., Micro-emulsion synthesis of monodisperse surface stabilized silicon nanocrystals. Chemical Communications 2005, (14), 1833-1835.

57_http://large.stanford.edu/courses/2007/ap273/hellstrom1/images/III_1_large.gif

58_http://www.ioffe.ru/SVA/NSM/Semicond/Si/bandstr.html

59_Wilcoxon, J. P.; Provencio, P. P.; Samara, G. A., Synthesis and optical properties of colloidal germanium nanocrystals. Physical Review B 2001, 64 (3), 035417.

60_http://www.ioffe.ru/SVA/NSM/Semicond/Ge/bandstr.html

61_Gresback, R.; Holman, Z.; Kortshagen, U., Nonthermal plasma synthesis of sizecontrolled, monodisperse, freestanding germanium nanocrystals. Applied Physics Letters 2007, 91 (9), 093119-3. 
62_ Stoldt, C. R.; Haag, M. A.; Larsen, B. A., Preparation of freestanding germanium nanocrystals by ultrasonic aerosol pyrolysis. Applied Physics Letters 2008, 93 (4), 043125-3.

63_Taylor, B. R.; Kauzlarich, S. M.; Delgado, G. R.; Lee, H. W. H., Solution Synthesis and Characterization of Quantum Confined Ge Nanoparticles. Chemistry of Materials 1999, $11(9), 2493-2500$.

64_Ma, X.; Wu, F.; Kauzlarich, S. M., Alkyl-terminated crystalline Ge nanoparticles prepared from NaGe: Synthesis, functionalization and optical properties. Journal of Solid State Chemistry 2008, 181 (7), 1628-1633.

65_Chiu, H. W.; Chervin, C. N.; Kauzlarich, S. M., Phase Changes in Ge Nanoparticles. Chemistry of Materials 2005, 17 (19), 4858-4864.

66_Lu, X.; Ziegler, K. J.; Ghezelbash, A.; Johnston, K. P.; Korgel, B. A., Synthesis of Germanium Nanocrystals in High Temperature Supercritical Fluid Solvents. Nano Letters 2004, 4 (5), 969-974.

67_ Wu, H. P.; Liu, J. F.; Wang, Y. W.; Zeng, Y. W.; Jiang, J. Z., Preparation of Ge nanocrystals via ultrasonic solution reduction. Materials Letters 2006, 60 (7), 986-989.

68_Warner, J. H. ; Tilley, R. D., Synthesis of water-soluble photoluminescent germanium nanocrystals. Nanotechnology 2006, 17 (15), 3745.

69_Prabakar, S.; Shiohara, A.; Hanada, S.; Fujioka, K.; Yamamoto, K.; Tilley, R. D., Size Controlled Synthesis of Germanium Nanocrystals by Hydride Reducing Agents and Their Biological Applications. Chemistry of Materials 2009, 22 (2), 482-486.

70_Smith, R. W., The [alpha](semiconductor) ag [beta](metal) transition in tin. Journal of the Less Common Metals 1985, 114 (1), 69-80.

71_Pavone, P.; Baroni, S.; de Gironcoli, S., alpha-beta phase transition in tin: A theoretical study based on density-functional perturbation theory. Physical Review $B$ 1998, 57 (17), 10421. 
72_Li, Z.; Tao, X.; Cheng, Y.; Wu, Z.; Zhang, Z.; Dang, H., A facile way for preparing tin nanoparticles from bulk tin via ultrasound dispersion. Ultrasonics Sonochemistry 2007, $14(1), 89-92$.

73_Lai, S. L.; Guo, J. Y.; Petrova, V.; Ramanath, G.; Allen, L. H., Size-Dependent Melting Properties of Small Tin Particles: Nanocalorimetric Measurements. Physical Review Letters 1996, 77 (1), 99.

74_Zou, C.-d.; Gao, Y.-I.; Yang, B.; Zhai, Q.-j., Size-dependent melting properties of Sn nanoparticles by chemical reduction synthesis. Transactions of Nonferrous Metals Society of China 2010, 20 (2), 248-253.

75_Huang, S.; Cho, E.-C.; Conibeer, G.; Green, M. A.; Bellet, D.; Bellet-Amalric, E.; Cheng, S., Fabrication and characterization of tin-based nanocrystals. Journal of Applied Physics 2007, 102 (11), 114304-6.

76_Huang, S.; So, Y. H.; Conibeer, G.; Green, M. A., In situ formation of tin nanocrystals embedded in silicon nitride matrix. Journal of Applied Physics 2009, 105 (12), 124303-5.

77_Jo, Y. H.; et al., Synthesis and characterization of low temperature Sn nanoparticles for the fabrication of highly conductive ink. Nanotechnology 2011, 22 (22), 225701.

78_Cui, Q.; Rajathurai, K.; Jia, W.; Li, X.; Gao, F.; Lei, Y.; Gu, Z., Synthesis of Single Crystalline Tin Nanorods and Their Application as Nanosoldering Materials. The Journal of Physical Chemistry C 2010, 114 (50), 21938-21942.

79_Wang, B.; Ouyang, G.; Yang, Y. H.; Yang, G. W., Anomalous thermal stability of cubic tin confined in a nanotube. Applied Physics Letters 2007, 90 (12), 121905-3.

80_Asom, M. T.; Kortan, A. R.; Kimerling, L. C.; Farrow, R. C., Structure and stability of metastable alpha-Sn. Applied Physics Letters 1989, 55 (14), 1439-1441.

81_Groves, S.; Paul, W., Band Structure of Gray Tin. Physical Review Letters 1963, 11 (5), 194.

82_King, H. W., Quantitative size-factors for metallic solid solutions. Journal of Materials Science 1966, 1 (1), 79-90. 
83_Pukite, P. R.; Harwit, A.; Iyer, S. S., Molecular beam epitaxy of metastable, diamond structure $\mathrm{Sn}_{\mathrm{x}} \mathrm{Ge}_{1-\mathrm{x}}$ alloys. Applied Physics Letters 1989, 54 (21), 2142-2144.

84_Taraci, J.; Tolle, J.; Kouvetakis, J.; McCartney, M. R.; Smith, D. J.; Menendez, J.; Santana, M. A., Simple chemical routes to diamond-cubic germanium--tin alloys. Applied Physics Letters 2001, 78 (23), 3607-3609.

85_Guo, Q.; Hillhouse, H. W.; Agrawal, R., Synthesis of Cu2ZnSnS4 Nanocrystal Ink and Its Use for Solar Cells. Journal of the American Chemical Society 2009, 131 (33), 1167211673.

86_Zdanowicz, T.; Rodziewicz, T.; Zabkowska-Waclawek, M., Theoretical analysis of the optimum energy band gap of semiconductors for fabrication of solar cells for applications in higher latitudes locations. Solar Energy Materials and Solar Cells 2005, 87 (1-4), 757-769.

87_Yang, Y.; Zhou, H.-P.; Hsu, W.-C.; Duan, H.-S.; Bob, B.; Yang, W.; Song, T.-b.; Hsu, C.J., CZTS Nanocrystals: A promising approach for next generation thin film photovoltaics. Energy \& Environmental Science 2013.

88_ Wang, J.; Xin, X.; Lin, Z., Cu2ZnSnS4 nanocrystals and graphene quantum dots for photovoltaics. Nanoscale 2011, 3 (8), 3040-3048.

89_Katagiri, H., Cu2ZnSnS4 thin film solar cells. Thin Solid Films 2005, 480-481 (0), 426432.

90_Todorov, T. K.; Tang, J.; Bag, S.; Gunawan, O.; Gokmen, T.; Zhu, Y.; Mitzi, D. B., Beyond 11\% Efficiency: Characteristics of State-of-the-Art Cu2ZnSn(S,Se)4 Solar Cells. Advanced Energy Materials 2013, 3 (1), 34-38. 
91_Akhavan, V. A.; Goodfellow, B. W.; Panthani, M. G.; Steinhagen, C.; Harvey, T. B.; Stolle, C. J.; Korgel, B. A., Colloidal CIGS and CZTS nanocrystals: A precursor route to printed photovoltaics. Journal of Solid State Chemistry 2012, 189 (0), 2-12.

92_ Wang, J.; Sun, S.; Peng, F.; Cao, L.; Sun, L., Efficient one-pot synthesis of highly photoluminescent alkyl-functionalised silicon nanocrystals. Chemical Communications 2011, 47 (17), 4941-4943.

93_Li, M.; Zhou, W.-H.; Guo, J.; Zhou, Y.-L.; Hou, Z.-L.; Jiao, J.; Zhou, Z.-J.; Du, Z.-L.; Wu, S.-X., Synthesis of Pure Metastable Wurtzite CZTS Nanocrystals by Facile One-Pot Method. The Journal of Physical Chemistry C 2012, 116 (50), 26507-26516.

94_Steinhagen, C.; Panthani, M. G.; Akhavan, V.; Goodfellow, B.; Koo, B.; Korgel, B. A., Synthesis of Cu2ZnSnS4 Nanocrystals for Use in Low-Cost Photovoltaics. Journal of the American Chemical Society 2009, 131 (35), 12554-12555.

95_Khare, A.; Wills, A. W.; Ammerman, L. M.; Norris, D. J.; Aydil, E. S., Size control and quantum confinement in Cu2ZnSnS4 nanocrystals. Chemical Communications 2011, 47 (42),

11721-11723. 


\section{Experimental techniques}

This chapter summarizes first the synthesis methods used for all the nanoparticles reported in this thesis. Secondly will be introduced all the techniques used to characterize the nanoparticles synthesized in this research.

\subsection{Synthetic techniques}

All the nanoparticles described in this thesis were synthesized via either the microemulsion synthesis techniques, high temperature decomposition techniques or hot injection methods. This section describes the synthetic techniques and the postformation treatments: surface modification and purification.

\subsubsection{Microemulsion synthesis}

The chemical reduction synthesis in reverse-micelles or microemulsion synthesis is a method carried out in a Schlenk tube at room temperature under a nitrogen atmosphere to avoid oxidation. This method is often described as water in oil system. Reverse micelles are formed by mixing a surfactant, either non-ionic aliphatic polyethers or quaternary ammonium cationic surfactants, presenting both hydrophobic and hydrophilic functional groups, in an organic solvent. The precursor, usually an anhydrous ionic salt, is chosen insoluble in the organic solvent and, when added to the mixture, it is confined within the reverse micelles. With addition of a reducing agent to the reaction mixture, the metal salt is reduced within the micelle and the metal atoms are reduced to their elemental state. When the concentration of atoms reaches the saturation level, the nucleation starts and particles are formed within the micelles, limiting the size of the nanoparticles. This technique was first developed for the synthesis of metallic nanoparticles. Semiconductor nanocrystals were first reported by the Wilcoxon's group for silicon and later Tilley et al reached the production of small monodisperse particles via this technique. ${ }^{1,2}$

In the case of silicon and germanium nanocrystals, a capping molecule is added to replace the surfactant attachment on the surface of the nanoparticles, to avoid 
complete oxidation. A capping molecule is necessary to impede aggregation and prevent oxidation. By exposure to air, tin nanoparticles synthesized by microemulsion system spontaneously form a thin oxide shell.

\subsubsection{Surface modification}

The use of a hydride reducing agent leads to the formation of nanoparticles with surfaces terminated by a hydrogen bond. As this bond is weak, by exposure to air, silicon and germanium are prone to oxidation. The surface termination can be tuned by reaction with a variety of organic molecules. The capping molecule can be either hydrophobic or hydrophilic, which leads to multiple possible applications for the quantum dots, including their use as biological markers. ${ }^{2}$ A hydrophilic surface is a challenge and essential for use in biology. Given that the surface area to volume ratio in a nanoparticle is large compared to bulk materials, the surface ligand can have a major impact on the properties of the nanocrystals.

In the case of silicon, the modification of the surface ()- $\mathrm{H}$ bond into a ()-C covalent bond occurs in presence of compounds with unsaturated C-C bonds. The unsaturation of an organic molecule for hydrosilylation has to be initiated using heat or light or Pt-based catalysts. In this research, the surface modification was conducted via UV-light irradiation. Swihart first reported the modification of the surface chemistry on silicon quantum dots by UV-light irradiation.,

\subsubsection{High temperature decomposition and hot injection synthesis}

The high temperature decomposition synthesis and hot injection synthesis methods are based on the decomposition of a metal precursor at elevated temperature, in the presence of a binding high boiling point organic solvent. The precursor is an organometallic compound or a metal salt. To avoid oxidation, the reaction is performed under an inert nitrogen atmosphere. The distribution of size of the nanocrystals can be limited by the use of a surfactant molecule or a highly coordinating solvent.

Both types of reaction are carried out in a three necked flask. In the high temperature decomposition, the precursors are mixed with the solvent at room temperature before heat treatment. In the hot injection synthesis process, the precursors are injected in the flask containing the hot solvent. The temperature is set higher than the decomposition 
temperature of the precursor, so that nuclei are created by the precursor decomposition and can agglomerate to form particles. The size and shape of the nanoparticles are controlled by changing parameters, such as temperature, concentration, solvents and precursors. The hot injection method allows a better size control of the nanoparticles because of a shortened nucleation time.

\subsubsection{Purification}

In order for the nanocrystals to be properly characterised, an extensive purification to remove by-products of the synthesis, including excess of surfactant and other impurities is required.

In this research, three main purification methods are investigated, depending of the characteristics of the produced nanoparticles. Tin, metal sulphides and CZTS nanoparticles are purified by separation using a centrifuge, while silicon or germanium quantum dots can be purified by size exclusion column chromatography or liquid-liquid phase separation technique.

As-synthesized tin, metal sulphides and CZTS nanoparticles are capped by a non-polar surfactant, such as oleylamine, and are stable in non-polar solvents. Mixed with a polar solvent such as methanol or ethanol, the particles can form a destabilized suspension of aggregated particles. The centripetal acceleration produced by the fast rotation of the centrifuge can separate two phases by the sedimentation principle. The solid nanoparticles are collected at the bottom of the centrifuge tubes, while the surfactant and other organic impurities remains in the solvent. The process can be repeated several times for an effective removal of the excess of impurities.

After the synthesis of silicon or germanium quantum dots, the solution contains excess of surfactant and lithium or sodium salts, resulting from the decomposition of the reducing agent that needs to be removed before characterization of the nanocrystals. $\mathrm{Si}$ and Ge NPs are too light to be separated using the centrifuge technique. The size exclusion column chromatography purification of nanoparticles has first been reported by Colvin et al for alkyl terminated cadmium selenide quantum dots. ${ }^{5,6}$ In this research, a Sephadex LH-20 liquid chromatography media was used. The spherical cross-linked dextran beads, with a size comprised between 25 and $100 \mu \mathrm{m}$ were placed in a column 
with methanol or toluene. By running the sample through the column, the different components can be separated depending on their size and the quantum dots can be collected by separating a fraction of the solvent (fig. 2.1) at the bottom of the column. QDs containing fractions are identified by their blue fluorescence.

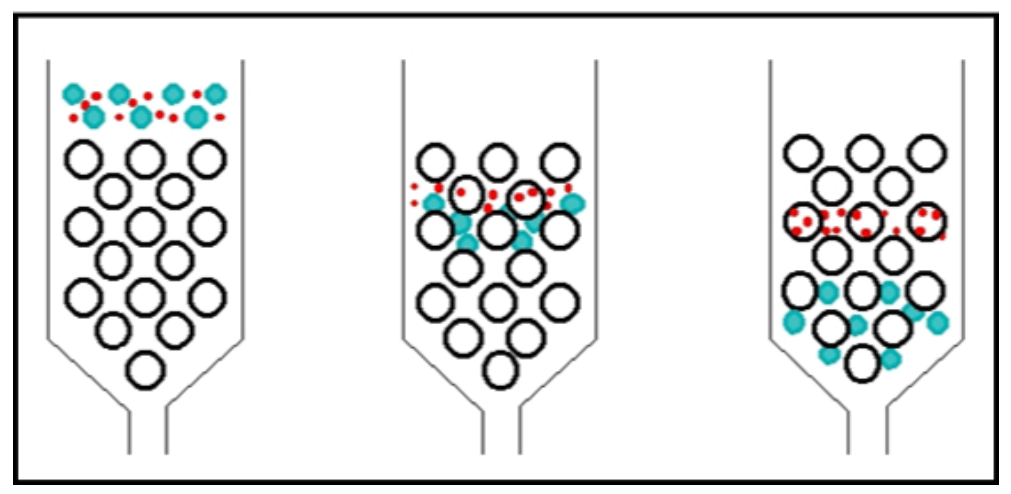

Fig. 2.1: Functioning of a size exclusion column

Another way to purify the Si and Ge nanoparticles is the liquid-liquid phase separation, reported by the Tilley group. ${ }^{2}$ This purification method is based on the polarity differences between the capping molecule attached to the surface of the quantum dots, the surfactant and other impurities. The nanoparticles capped with non-polar molecules, such as alkyl-terminated are washed several times with a mixture of hexane and $\mathrm{N}$-methylformamide. The hexane phase will contain the nanocrystals and all polar impurities will be attracted in the polar phase. The isolation of the non-polar phase will separate the quantum dots from the impurities. The process has to be repeated several times to achieve a complete separation of the nanoparticles.

\subsection{Characterization techniques}

\subsubsection{Transmission electron microscopy (TEM)}

Transmission Electron Microscopy (TEM) is a powerful technique for structural and morphological characterization of nanomaterials. The basic principle of a TEM is analogous to an optical microscope, except that the light is replaced by a stream of electrons. Imaging nanoparticles is not possible with an optical microscope due to magnification limitation. The highest resolution obtainable for any microscope is half of the wavelength used and the electrons have a much shorter wavelength than visible 
light, giving the electron microscopes a much higher resolution than optical microscopes. $^{7}$

Most electron microscopes contain a thermionic source in the form of a tungsten filament or a lanthanum hexaboride $\left(\mathrm{LaB}_{6}\right)$ single crystal as an electron source that produces an electron beam. Field emission TEMs exploit a tungsten needle subjected to a strong electrostatic field. ${ }^{8}$ The wavelength of an electron is directly correlated to the accelerating voltage applied through the relation:

$$
\lambda \approx \frac{h}{\sqrt{2 m e V}}
$$

Where $\lambda$ is the electron wavelength, $h$ is the Planck constant, $m$ is the mass of the electron and $\mathrm{eV}$ is the charge of an electron.

A TEM is mostly composed of one vertical column. A schematic of a typical TEM set-up is presented in figure 2.2. The electron source is located at the top and the beam of electrons is condensed and oriented by means of condensers and a series of electromagnetic lenses. An image of the sample is projected on a fluorescent screen at the bottom of the column. The system is maintained under high vacuum conditions to prevent undesirable scattering of the imaging beam of electrons. Samples are placed on a specimen holder in the direct path of the electron beam. Electrons encountering no objects are not subject to deviations, but e- are diffracted by the sample, with elastic and inelastic scattering. TEM exploits electrons scattered elastically, which conserve their energy. Scattering phenomena are related to the atomic number of each element, leading to an enhanced contrast for heavy elements; high atomic number elements are more prone to occlusion or absorption of imaging electrons, resulting in a darker area on the image. Inelastically scattered electrons are exploited by other techniques, such as energy dispersive X-ray spectroscopy (section 2.2.3). ${ }^{9}$

Transmission electron microscopy offers important advantages over other techniques in the characterization of nanocrystals. Nevertheless, the resolution of TEM suffers from limitations due to aberrations created along the column. Any imperfection in the objective lens will result in an astigmatism. Also, the variation of electron energy within 
the beam leads to chromatic aberrations, which is a distortion of the image due to a difference in focal points of the magnetic lenses for different wavelengths. ${ }^{10}$

The image can also be viewed and recorded with a digital camera or printed on a film by an analogue camera.

For this project, a JEOL 2010 transmission electron microscope was used. The acceleration voltage is set at $200 \mathrm{kV}$. The electron source is a $\mathrm{LaB}_{6}$ single crystal filament. Samples are dispersed into an organic solvent and a drop is deposited onto an amorphous carbon coated TEM grid.

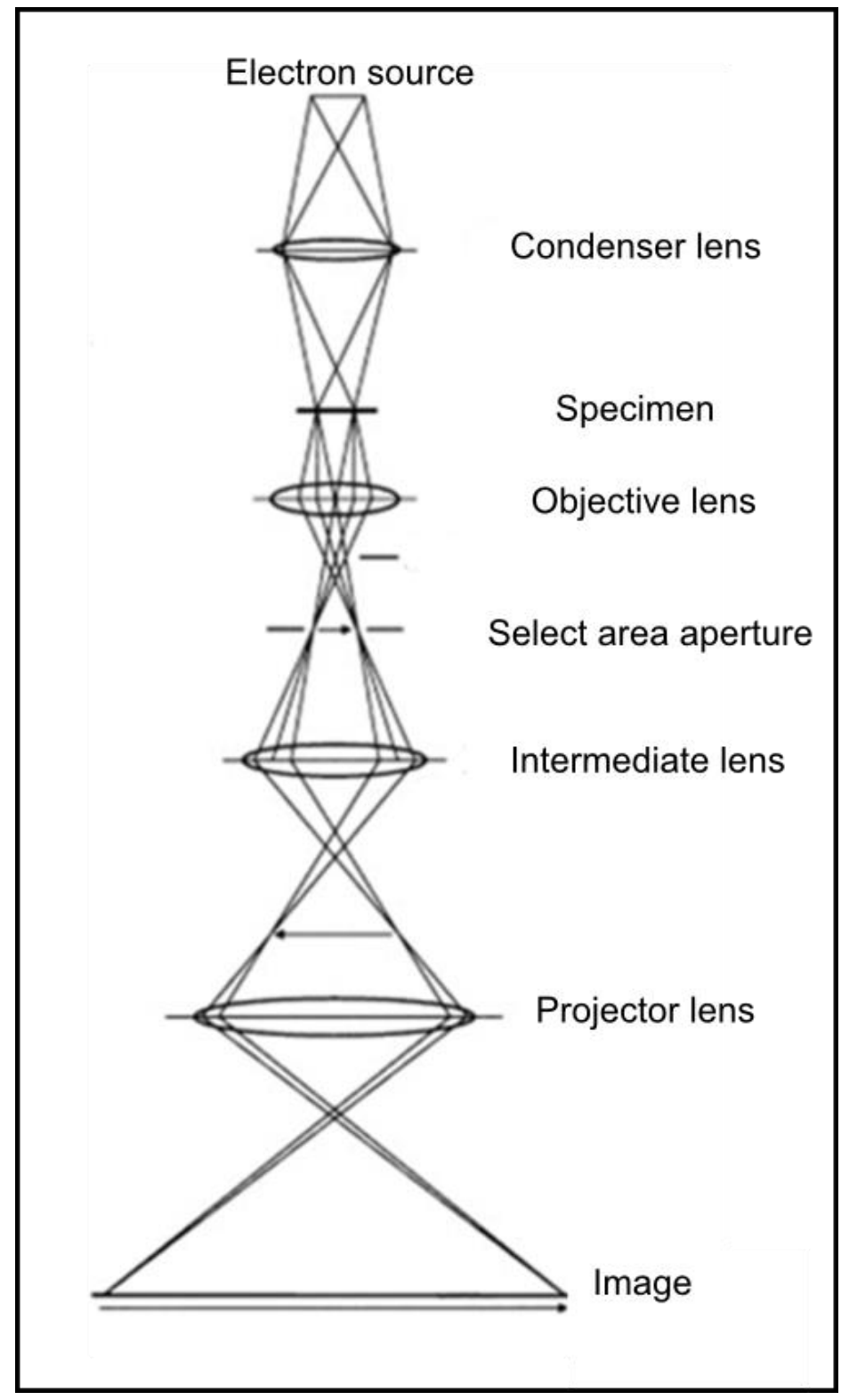

Fig. 2.2: Schematic of a typical set up of a TEM instrument. ${ }^{11}$ 
Low resolution TEM images enables to characterize nanoparticles by their size and shape. A size distribution can be determined by measuring particles over different areas of the sample using the program ImageJ and the distribution is plotted in the form of a histogram presenting the measured sizes by frequency count. The difference between two phases can be determined by a difference in contrast on the image. High resolution TEM images can be obtained at high magnifications (400.000x or higher). With high resolution imaging, it becomes possible to image at the atomic level and crystalline lattice fringes can be observed. The spacing between those lattice fringes gives information about the atomic arrangement and the crystal structure of a nanoparticle.

\subsubsection{Scanning Electron Microscopy (SEM)}

Scanning Electron Microscopy (SEM) is another electron microscopy techniques widely used in the nanoscale technology. Contrarily to the TEM, the SEM allows the imaging of samples in three-dimensions. It provides information about the morphology and the topography of a sample. ${ }^{12}$ At Victoria University, we use a JEOL 6500F Field-emission SEM, with a range of resolution from $25 x$ to $500,000 x$.

A tungsten filament is used as an electron source. The beam of electron is accelerated by a potential difference and directed onto the sample. When the electron beam encounters matter, three main interactions give detectable signals. The inelastic scattering of the incident electrons produces secondary electrons that give a topographic image of the sample. The incident electrons can also be scattered elastically by the nucleus of the atoms. The produced electrons are called back-scattered electrons. This interaction depends on the atomic number of the atoms, giving an elemental contrast on the image obtained with the backscattered detector. ${ }^{13}$ Finally, $\mathrm{X}$ rays are emitted by relaxation of electrons after excitation by the incident e- beam. These X-rays are detected by an EDS detector (see section 2.2.3).

\subsubsection{Energy dispersive X-ray spectroscopy (EDS)}

The Energy Dispersive X-ray spectroscopy (EDS) is a technique used to determine quantitatively the elemental composition of a material by giving a percentage of each element present in a desired area of the sample. ${ }^{14}$ The EDS detector is associated to the 
electron microscopes, TEM or SEM. When the electron beam hits the sample, the high energy electrons give enough energy to remove an electron from an inner shell of an atom, creating a hole. An electron from the outer shell will replace the vacancy created, releasing some of its energy by producing a photon of $\mathrm{X}$-ray radiation. The EDS technique is based on the detection of those X-rays that are characteristic of each element of the periodic table. The recorded spectra present peaks for each element detected. Copper and carbon are usually present on the spectra due to the carbon coated copper grid used for TEM imaging.

EDS can also be an area specific technique, called EDS mapping. The EDS data is synchronized with an SEM or TEM image to provide an elemental image.

However, with EDS, some constraints are notable. EDS can only detect elements heavier than Beryllium. The accuracy of the measured elemental percentage value can have a deviation up to $\pm 5 \%$. EDS detector have also a limit to the numbers of $X$-rays that can be analyzed at a time, favouring the lower emissions rather than the higher emissions. ${ }^{15}$

\subsubsection{Diffraction techniques: X-ray powder diffraction (XRD) and selected area electron diffraction (SAED)}

Crystals are ordered materials defined by atomic planes. When a radiation, either X-rays or electron beam, is directed onto a crystal, the radiation can be scattered by the atoms on a specific crystal planes. Bragg's law establishes a relationship between the different atomic spacings in the crystal, the wavelength of the radiation and the angle of the diffracted beam. It can be described mathematically by:

$$
\mathrm{n} \lambda=2 \mathrm{~d}_{\mathrm{hk}} \sin \theta
$$

where $n$ is an integer representing the order of reflexion, $\lambda$ is the wavelength, $d_{h k l}$ is the interplanar distance and $\theta$ is the diffraction angle. $h, k$ and $/$ are called Miller indices, and correspond to the directions of the planes along the reciprocal lattice. 


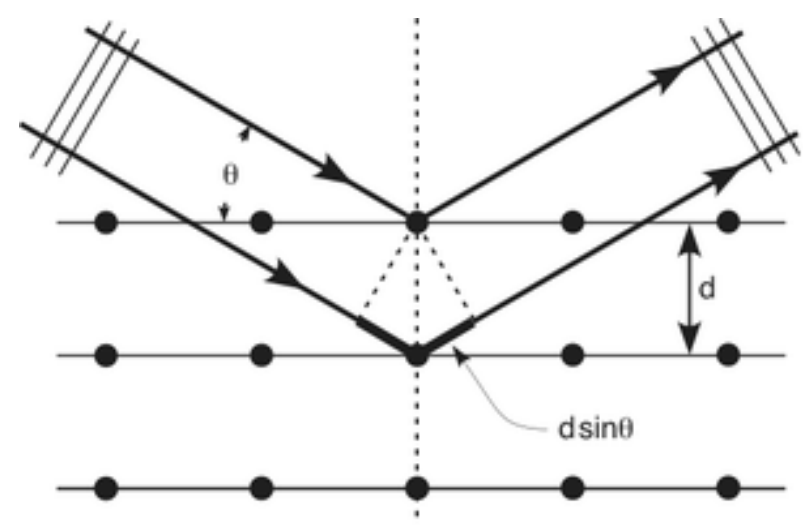

Fig. 2.3: Illustration of Bragg's law. ${ }^{16}$

Powder X-ray diffraction is measured on a solid sample packed in a holder. The surface of the sample must be flat to avoid aberrations. The XRD machine used in this research is the PANalytical X'Pert Pro Multi-Purpose diffractometer with a copper $\mathrm{K}_{\alpha}$ source $(\lambda=1.5406 \AA)$. To measure the diffraction created by a sample, the source and detectors are rotated by the same angle $\theta$. The spectra obtained represent the intensity of the reflection in function of the angle $2 \theta$. The structure of the sample can be determined by comparison to a JCPDS database. The spinner stage was used to remove orientation aberrations.

The average particle size can also be determined by diffraction measurements. The decreasing size of the particles induces a broadening of the diffraction peaks. By measuring the FWHM (Full width at half maxima), it is possible to estimate the crystallite sizes of a sample using the Scherrer equation, given by:

$$
\tau=\frac{\mathrm{K} \lambda}{\beta \cos \theta}
$$

Where $\tau$ represents the crystallite size, $\mathrm{K}$ is the shape factor (constant estimated at 0.9 for spherical particles), $\lambda$ is the wavelength of radiation, $\theta$ is the reflection angle and $\beta$ is the full-width at half maximum of the peak. ${ }^{17}$

XRD is limited in the resolution of nanostructures. For crystals with around 100 atoms or fewer, XRD would discern with difficulty the crystal orientations. Also, a peak overlay may happen in multiple phases samples, complicating the phase or crystal structure identification. 
The selected area electron diffraction technique is associated with the TEM. The electrons are scattered by the planes of atoms in a limited field of view. The diffraction image is obtained by changing the objective aperture used for imaging for the intermediate aperture. The diffraction of the electron beam by the sample gives rings or a series of spots. If the area selected for diffraction is a single crystal, the image will be presenting spots that can be measured and compared to the reciprocal lattice of the crystal. If the area presents multiple crystal orientations, for example for small and densely packed nanoparticles, the SAED diffraction image will be in the form of rings. The diameters of the rings correspond to atomic d-spacings. Rings diameter can be measured and d-spacing are calculated depending on the camera length, the electron wavelength and the diameter of the rings. The rings are then indexed by comparison with a JCPDS reference database.

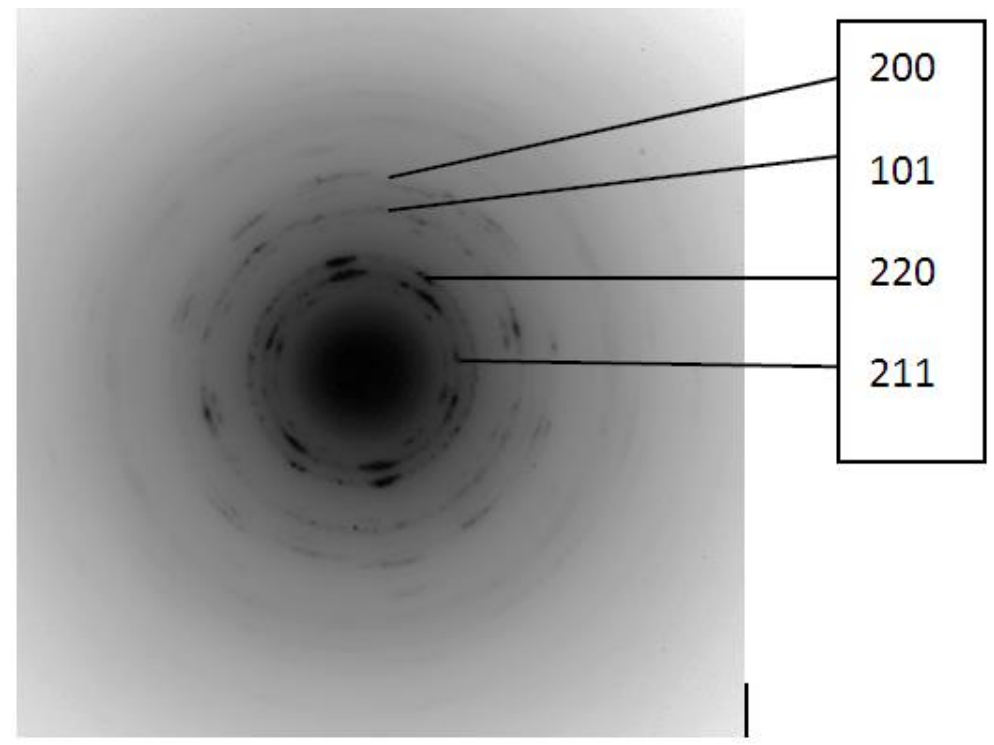

Fig. 2.4: SAED pattern of $\beta$-tin nanoparticles with the indexation of each diffraction rings to the $d$-spacings corresponding to the (200), (101), (220) and (211) orientations.

Electron diffraction can be taken by a digital camera or on photographic film with an analogue camera.

\subsubsection{Fourier Transform Infrared Spectroscopy}

Fourier Transform Infrared Spectroscopy (FTIR) is a vibrational spectroscopy technique that characterizes qualitatively a material by identifying the chemical bonds in the material. ${ }^{18}$ When an infrared radiation, typically in the range $10,000-100 \mathrm{~cm}^{-1}$, 
encounters matter, it produces molecular vibrations. With each bond type absorbing a different wavelength, a compound can be identified by the IR frequencies absorbed. After a Fourier transform treatment of the signal, a typical spectrum of the material can be displayed. For the study of semiconductor nanoparticles, FTIR spectroscopy allows to determine the chemical structure of the capping molecules and their attachment to the surface of the nanocrystals. FTIR spectroscopy is especially important for the study of silicon and germanium quantum dots whose surface can be modified by hydrophilic or hydrophobic molecules.

The infra-red spectrometer used in this research project is a Bruker Tensor 27. Samples are prepared by mixing a nanoparticles suspension in a volatile organic solvent into $\mathrm{KBr}$, then drying the organic solvent for a few minutes in a $100^{\circ} \mathrm{C}$ oven and finally forming a pellet.

\subsubsection{Nuclear Magnetic Resonance}

Nuclear Magnetic Resonance (NMR) is a powerful non-destructive analytical tool that permits to ascertain molecular structures including relative configuration, relative and absolute concentrations, and even intermolecular interactions of a sample. In this research, we use NMR to assess the surface chemistry of silicon quantum dots.

When an atom is placed in a magnetic field, its electrons circulate about the direction of the applied magnetic field. This circulation causes a small magnetic field at the nucleus which opposes the externally applied field. The specific resonance frequency depends on the intensity of the applied magnetic field and on the nuclei. ${ }^{19}$

The electron density around each nucleus in a molecule varies according to the types of nuclei and bonds in the molecule. The magnetic field of each nucleus, opposed to the applied magnetic field, will vary depending on its environment. This is called the chemical shift phenomenon. ${ }^{20}$

${ }^{1} \mathrm{H}$ NMR enables to distinguish the different configurations of the protons and to estimate the relative position of every hydrogen atom in a molecule.

${ }^{1} \mathrm{H}$ NMR spectra were recorded on a Varian Unity Inova $500 \mathrm{MHz}$ spectrometer. The nanoparticles must be thoroughly dried from any organic solvent. After removing most 
of the solvent using a rotary evaporator, the samples were dried under vacuum overnight before being dissolved in a deuterated solvent.

\subsubsection{Optical characterization}

\subsubsection{UV-Vis spectroscopy}

Ultraviolet-Visible spectroscopy is a technique used to determine the absorption wavelengths of a substance in solution or deposited onto a transparent substrate. For nanostructured materials, UV-Vis spectroscopy can be used to characterize the effects of the quantum confinement. Gold or silver nanoparticles for example absorb different colours according to their size distribution. A blue shift in the absorption maxima is often observed by decreasing size of nanomaterials compare to the bulk values.

This technique is also useful for semiconducting materials. The edge of the absorption spectrum permits to get an estimation of the bandgap energy. When a photon in the electromagnetic wave hits the sample, it transfers its energy to the electrons in the Valence band. Only photons which energy is equal or higher than the bandgap of the material can be absorbed. So the bandgap can be estimated by the Einstein equation:

$$
\mathrm{E}_{\mathrm{g}}=\mathrm{hc} / \lambda
$$

Where $h$ is the Planck's constant, $c$ is the velocity of light and $\lambda$ is the maximum absorption wavelength.

More accurately, the $(A h v)^{2}$ values, with $A$ being the absorption coefficient, $h$ the Planck constant and $v$ the wavenumber, can be plotted as a function of photon energy to get a better an evaluation of the bandgap value by fitting an asymptotic line on the decreasing part of the curve and extrapolating to zero.

The measurements are performed on diluted samples in organic solvents placed in quartz cuvettes or on samples deposited onto UV-transparent glass substrates. A Varian Cary 50 double beam Bio UV-Visible spectrometer was used to record the UV-Vis absorption measurements in this research. 


\subsubsection{Photoluminescence spectroscopy}

Photoluminescence spectroscopy is a technique used to determine the light emission processes in a fluorescent or phosphorescent sample. The solution is excited by a light radiation and the wavelengths emitted by the sample are recorded. The photoluminescence (PL) phenomenon occurs when an excited electron releases its energy by recombination with the hole in the valence band and thus emitting a photon. ${ }^{21}$ This light emission can be detected and measured using a spectrofluorometer.

A Horiba Jobin Yvon Fluorolog-3 spectrofluorometer was used for PL measurements in this research. As well as for the UV-Vis absorption measurements, measurements are carried out on samples diluted in organic solvents placed $1 \mathrm{~cm}$ in four sided quartz cuvettes or on sample deposited onto Spectrosil glass substrates.

The majority of the potential applications for semiconductor nanoparticles, including group IV quantum dots, involve their unique optical properties. These properties can be characterized by the combination of UV-Vis spectroscopy and PL measurements.

A PL emission spectrum provides information about the wavelength emitted by a sample depending on the excitation wavelength. On the contrary, a PL excitation (PLE) spectrum reports which wavelengths provoke a given emission, by exposing a sample to the photoluminescence wavelength. PLE spectra are recorded with the same spectrofluorometer than PL measurements.

\subsubsection{Time-resolved photoluminescence spectroscopy}

Time-resolved photoluminescence measures the decay of the photoluminescence. It allows to estimate the time in which the electrons recombined with a hole in the Valence state after excitation.

Using the following exponential decay function,

$$
I(t)=I_{o} \exp (-t / \tau)^{6}
$$

Where $\tau$ is the characteristic PL lifetime and $\beta$ is the dispersion exponent, the PL decay of fluorescent materials can be determined. ${ }^{22} \beta$ is used to approximate the decay function for quantum dots to a single exponential equation and palliate the fluctuations of single dot emission intensity, known as blinking. ${ }^{23}$ 
An estimation of the PL lifetime gives an indication of the type of transition creating the emission phenomenon. A fast recombination ( $\leq 10 \mathrm{~ns})$ is characteristic of a radiative decay from the direct recombination of an exciton. It is usually associated to the direct or pseudo-direct bandgap transition in semiconductors nanocrystals (figure 1.4). PL decays in the order of $\mu$ s or ms are of non-radiative nature, suggesting a defect driven photoluminescence.

This technique was used at the University of Massachusetts-Amherst, USA with the collaboration of Boqian Yang from Michael Barnes's group. Boqian measured the PL lifetime of my silicon quantum dots in solution using his experimental set up.

All the techniques described in this chapter were used in the research described in the following chapters. At the exception of the PL lifetime measurements, all the characterization presented was solely done by me. I would like to thank Victoria University for the access to the all the facilities used in this research. 


\subsection{References}

1_Wilcoxon, J. P.; Samara, G. A., Tailorable, visible light emission from silicon nanocrystals. Applied Physics Letters 1999, 74 (21), 3164-3166.

2_ Warner, J. H.; Hoshino, A.; Yamamoto, K.; Tilley, R. D., Water-Soluble Photoluminescent Silicon Quantum Dots. Angewandte Chemie International Edition 2005, 44 (29), 4550-4554.

3_Sato, S.; Swihart, M. T., Propionic-Acid-Terminated Silicon Nanoparticles: Synthesis and Optical Characterization. Chemistry of Materials 2006, 18 (17), 4083-4088.

4_Erogbogbo, F.; Yong, K.-T.; Roy, I.; Xu, G.; Prasad, P. N.; Swihart, M. T., Biocompatible Luminescent Silicon Quantum Dots for Imaging of Cancer Cells. ACS Nano 2008, 2 (5), 873-878.

5_ Krueger, K. M.; Al-Somali, A. M.; Falkner, J. C.; Colvin, V. L., Characterization of nanocrystalline CdSe by size exclusion chromatography. Anal. Chem. 2005, 77 (11), 3511-3515.

6_Yu, W. W.; Chang, E.; Falkner, J. C.; Zhang, J.; Al-Somali, A. M.; Sayes, C. M.; Johns, J.; Drezek, R.; Colvin, V. L., Forming Biocompatible and Nonaggregated Nanocrystals in Water Using Amphiphilic Polymers. Journal of the American Chemical Society 2007, 129 (10), 2871-2879.

7_ B. Fultz and J. M. Howe, "Transmission Electron Microscopy and Diffractometry of Materials" Springer Berlin Heidelberg 2008

8_ Williams, D.B., C.N. Carter, "Transmission Electron Microscopy: A Textbook for Materials Science. " Springer US 2009

9_ De Graef, M., "Introduction to Conventional Transmission Electron Microscopy", Cambridge University Press, 2003

10_FEl: An Introduction to Electron Microscopy 2010 
11_http://barrett-group.mcgill.ca/tutorials/nanotechnology/nano02.htm

12_ Midgley, P. A., The principles of microscopy. Materials Today 2006, 9 (10), 57.

13_Danilatos, G. D.; Robinson, V. N. E., Principles of scanning electron microscopy at high specimen chamber pressures. Scanning 1979, 2 (2), 72-82.

14_Schneider, R., Energy-Dispersive X-Ray Spectroscopy (EDXS). In Surface and Thin Film Analysis, Wiley-VCH Verlag GmbH \& Co. KGaA: 2011; pp 293-310.

15_http://micron.ucr.edu/public/manuals/EDS-intro.pdf

16_http://www.sacclab.com/techniques.html

17_prism.mit.edu/xray/CrystalSizeAnalysis.ppt

18_http://chemistry.oregonstate.edu/courses/ch361-464/ch362/irinstrs.htm

19

http://www2.chemistry.msu.edu/facilities/nmr/handouts/DH\%20NMR\%20Basics.pdf

20_Hornak, J.P., " The basics of NMR" 1997

21_Lakowicz, J.R, "Principles of Fluorescence Spectroscopy", $3^{\text {rd }}$ edition, Springer 2006

22_Millar, D. P., Time-resolved fluorescence spectroscopy. Current Opinion in Structural Biology 1996, 6 (5), 637-642.

23_Chae, W.-S.; Ung, T. D. T.; Nguyen, Q. L., Time-resolved photoluminescence and photostability of single semiconductor quantum dots. Advances in Natural Sciences: Nanoscience and Nanotechnology 2013, 4 (4), 045009. 


\section{Origin of the photoluminescence in silicon quantum dots}

Nanostructures of silicon are known to be photoluminescent in contrast to their bulk counterparts. The origin of this photoluminescence is still heavily debated in the literature. In this chapter will be investigated the optical properties of quantum dots and offer a theory towards their origin.

The synthetic method to produce hydrogen terminated silicon quantum dots is based on the reverse micelle technique. The synthesis involves the reduction of a silicon salt using a hydride reducing agent. Wilcoxon and co-workers were the first to report the synthesis of silicon quantum dots using a reverse micelles technique. ${ }^{1}$ Tilley and co-workers refined this method to obtain monodispersed silicon quantum dots. ${ }^{2}$ The nanocystals growth is followed by the surface modification with organic molecules using UV-light to activate the hydrosilylation. After a purification process, the particles were characterized structurally and optically to propose an answer to the origin of the photoluminescence. Pursuing the work reported by Shiohara et al, the influence the reducing agent on the size of the nanocrystals and on their optical properties was then investigated, followed by the study of the role played by the surface chemistry of the silicon quantum dots, via the study of three different capping molecules: allylamine, 1hexene and 1-dodecene. ${ }^{3}$

The nanoparticles were characterized by XRD, EDS, TEM and the size distributions were calculated from the low resolution TEM images. The surface attachments of the nanocrystals were characterized using FTIR and NMR spectroscopies. The optical properties of the quantum dots in solution were assessed using UV-Visible, photoluminescence and time-resolved photoluminescence spectroscopies. 


\subsection{Synthesis of silicon quantum dots}

The synthesis described in this section is using the reverse micelles principle. Several methods have previously been reported regarding the synthesis of silicon nanocrystals. Veinot and co-workers reported the thermal decomposition of hydrogen silsesquioxane (HSQ) producing oxide embedded silicon nanocrystals that were then etched using a hydrofluoric acid bath solution to obtain hydride surface-terminated silicon quantum dots. ${ }^{4,5}$ Kauzlarich et al produced silicon nanocrystals through the decomposition of Zintl salts via solution processor microwave assisted reactions. ${ }^{6,7}$ Korgel et al developed a silicon quantum dots synthesis using supercritical conditions. ${ }^{8,9}$

After the synthesis of hydride terminated silicon quantum dots, the particles undergo a surface passivation process to render the nanocrystals stable in an ambient environment. Finally the purification process used to free the particles from surfactant molecules and other impurities generated during the synthetic route will be described.

\subsubsection{Hydride terminated silicon quantum dots synthesis}

The synthesis of silicon in this study is based on the chemical reduction of a silicon salt by the mean of a hydride reducing agent. The reaction mechanism can be described as follows:

$$
2 \mathrm{SiX}_{4}+2 \mathrm{MBH}_{4} \rightarrow \mathrm{Si}+2 \mathrm{MX}+2 \mathrm{BX}_{3}+\mathrm{H}_{2}+\mathrm{SiH}_{4}
$$

Where $X$ is a halogen, typically chloride and $M$ represents an alkali metal, either sodium or lithium. This reaction must be carried out in a controlled oxygen free atmosphere to avoid oxidation. Also the production of silane and hydrogen gas must be taken into consideration and the reaction must be conducted with care. The rising concentration of $\mathrm{Si}$ monomers in the solution as the reduction of $\mathrm{SiCl}_{4}$ takes place allows the monomers to agglomerate and form nanoparticles. The particle growth is controlled by the confinement into the reverse micelles formed by the surfactant molecule in the solvent media.

Silicon quantum dots are highly sensitive to oxidation without surface passivation. Before the surface passivation, the silicon quantum dots surface is terminated by a Si-H 
bond, and is highly unstable in an oxygen containing environment, like air. Characterising silicon nanoparticles with hydride bonds on the surface requires the use of in-situ equipment that was not available for this study. The synthesized silicon nanocrystals were fully characterized after surface passivation.

\section{Experimental}

Either $0.5 \mathrm{~g}$ of $\mathrm{C}_{12} \mathrm{E}_{5}$ or $0.5 \mathrm{~g}$ of TOAB was placed in a Schlenk tube sealed with a septum. The experimental set-up is presented in figure 1 . The system was degassed under vacuum for 2 minutes then purged with nitrogen for 2 minutes. The degas/purging process was repeated three time to ensure that no oxygen was remaining in the reaction vessel. $50 \mathrm{~mL}$ of anhydrous solvent was injected in the mixture (hexane with $\mathrm{C}_{12} \mathrm{E}_{5}$ or toluene with $\left.\mathrm{TOAB}\right)$. The solution was thoroughly stirred for ten minutes to render the mixture homogeneous and allow the surfactant to arrange itself in the micelle form. Then, $0.3 \mathrm{~mL}$ of silicon tetrachloride was injected swiftly in the Schlenk tube. After 10 minutes of active stirring, $4 \mathrm{~mL}$ of reducing agent (either lithium borohydride or sodium borohydride) was swiftly injected into the solution. In the formaly clear transparent solution is created a white precipitate. The reaction is left to run under nitrogen for at least one hour.

This reaction must be conducted with precaution due to the production of hydrogen gas and silane. 


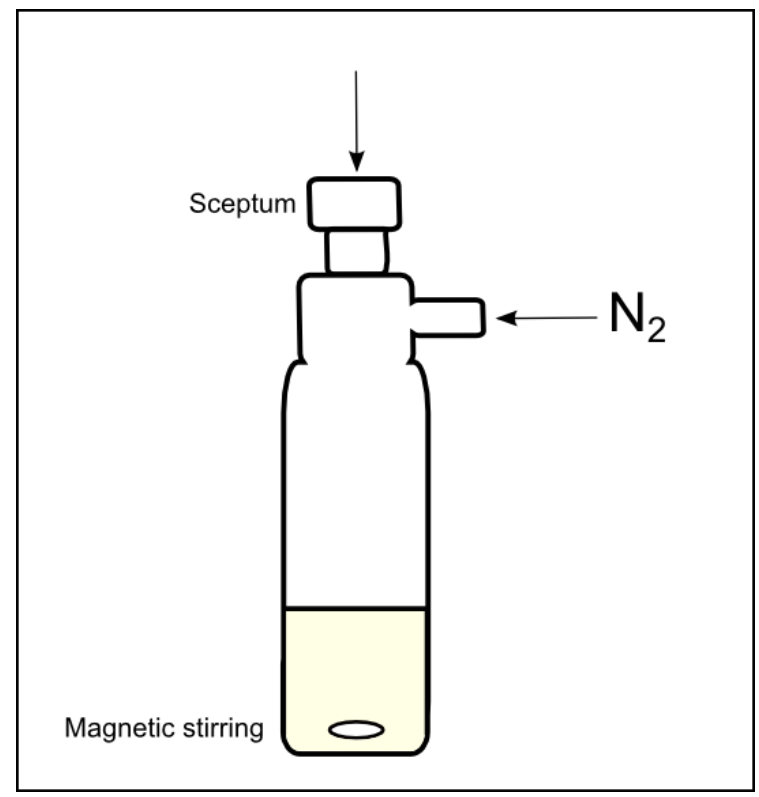

Figure 3.1: Schlenk tube set up for the synthesis of silicon nanocrystals

At this stage the solution contains Si nanocrystals with a hydride surface termination which oxidizes rapidly if exposed to air. The surface needs to be passivated via hydrosilylation to render the particles stable in air and common solvents.

\subsubsection{Surface passivation}

To prevent the oxidation of the silicon nanocrystals, the surface of the nanoparticles is passivated with organic molecules. Grignard or alkyl reagents can be used to obtain alkyl terminated quantum dots. ${ }^{10,11}$ Rogozhina et al reported the carboxyl functionalization of silicon quantum dots through thermal hydrosilylation at $140^{\circ} \mathrm{C}$ in presence of a methyl ester. ${ }^{12}$ Korgel and co-workers described the alkoxy-functionalization on Si NPs via thermal decomposition of diphenylsilane in supercritical solvents. ${ }^{13}$ Warner et al reported surface modification of silicon quantum dots using a platinum catalyst. ${ }^{2}$ This method is suitable for organic capping molecules with various functional groups, but former studies conducted by Shiohara et $a l$ and the Tilley group related the production of platinum impurities due to the reduction of the platinum catalyst with the excess reducing agent necessary for the silicon quantum dots reaction. ${ }^{14}$ Alternatively, the method of hydrosilylation used in this study is based in the photo activation under UV 
light, reported first by Swihart et al. ${ }^{15,16}$. The exposition to UV-light initiate the generation of a covalent bonding between silicon atoms and the organic molecule. A scheme of the hydrosilylation process is presented in figure 2.

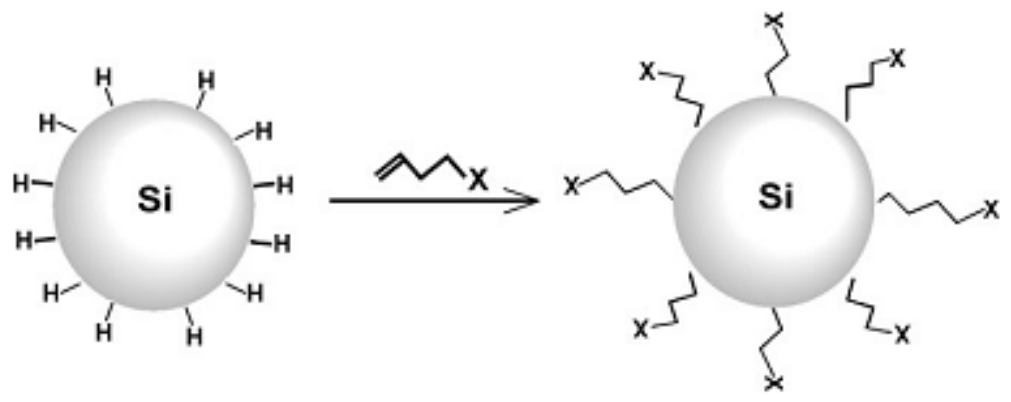

Figure 3.2: Scheme of the procedure used to modify the surface capping molecule of silicon quantum dots from hydrogen terminated to polar or non-polar groups (X). ${ }^{17}$

\section{Experimental}

The hydride terminated-Si quantum dot solution synthesized in section 3.1.1 was contained in a Schlenk tube sealed with a septum. The magnetic stirring was stopped so the white precipitate of salts, which are side products of the reduction of $\mathrm{SiCl}_{4}$ by the hydride reducing agent, could settle at the bottom of the tube. A $50 \mathrm{~cm}$ high quartz tube was sealed with a septum and freed of oxygen by three cycles of degassing under vacuum followed by purging with a nitrogen flow. Using an air tight syringe, the solution was transferred from the Schlenk tube to the quartz tube. Then $2 \mathrm{~mL}$ of capping agent (allylamine, 1-hexene or 1-dodecene) was injected into the solution. To homogenize the solution, the tube was shaken by hand. Silicon quantum dots do not precipitate but stay suspended in solution. The quartz tube was closed tightly to keep the nitrogen atmosphere and placed in a Rayonet UV-lamp set-up with a $254 \mathrm{~nm}$ wavelength. After UV treatment for four hours, the solution can be exposed to air without risking the oxidation of the silicon quantum dots.

\subsubsection{Purification}

After surface passivation, the silicon quantum dots are not sensitive to oxidation and the solution can be exposed to air. To remove side products, such as lithium or sodium salts 
or excess surfactant, the nanoparticles need to be purified. Two methods have been used in this research. Solvent separation deals with the polarity differences between the particles and the surfactant impurities by dissolving them in two non-miscible solvents. This method has first been reported by Tilley et al in 2005 for silicon nanocrystals. ${ }^{2}$ This purification technique is generally used for non-polar capping molecules on the surface of silicon quantum dots. Size exclusion column chromatography is the other technique used here to purify nanocrystals. A nanocrystal solution is slowly passed through a column of silica beads, thus separating the particles by their size. ${ }^{18,19}$ This method for nanocrystals purification was first been reported for nanoparticles of cadmium selenide by Colvin et al in $2005 .^{18}$

\section{Experimental}

The nanocrystals capped with a polar molecule (allylamine) were separated from impurities by size exclusion column chromatography. The solution obtained after the capping process (see section 3.1.2) is transferred into a round bottom flask and the solvent is evaporated under vacuum using a rotary evaporator and reduced to approximately $10 \mathrm{~mL}$. The solution was filtered using a Millipore $0.22 \mu \mathrm{m}$ syringe filter before being concentrated to a $2 \mathrm{~mL}$ solution. After five minutes of homogenization in an ultrasonic bath, the solution was then put on top of the column $(\phi=1 \mathrm{~cm}, 41.0 \mathrm{~cm})$, containing Sephadex gel LH-20 (beads size 25-100 $\mu \mathrm{m}$ ) as the stationary phase in HPLC grade methanol. The flow rate was set to one drop every four seconds, and fractions were collected every 50 drops. Each fraction was checked for luminescence with a handheld UV lamp (365 $\mathrm{nm}$ ). Luminescent fractions were collected and concentrated to $1 \mathrm{~mL}$ under vacuum. The size exclusion process was repeated two or three times to obtain pure surface passivated silicon quantum dots.

The nanocrystals with a non-polar surface can be purified by two or three cycles of size exclusion column chromatography using toluene as the solvent for the Sephadex LH-20 gel. The alkene capped silicon nanocrystals can also be purified by liquid-liquid phase separation chromatography. The solution after UV treatment is concentrated down to about $10 \mathrm{~mL}$ and filtered using a Millipore $0.22 \mu \mathrm{m}$ syringe filter after five minutes in an ultrasonic bath. The solution is placed in a separatoring funnel and $15 \mathrm{~mL}$ of Nmethylformamide is added. The funnel was sealed at the top and shaken energetically 
with frequent gas release to avoid an air build-up in the funnel. The funnel was left to rest until the two phases were completely separated (at least 15 minutes). After three washes using $\mathrm{N}$-methylformamide, the polar phase was changed for water. Three or more washes were necessary to remove most impurities from the silicon quantum dots solution.

After this stage, silicon nanocrystals can be characterized with various spectroscopy techniques and transmission electron microscopy.

\subsubsection{Structural characterization}

In this section will be first described the characterization of the silicon core of the nanoparticles. We used a sample of silicon quantum dots capped with allylamine synthesized using $\mathrm{LiBH}_{4}$ for the structural characterization and the determination of the optical properties later.

Using X-ray diffraction, we can evaluate the crystal structure of our nanoparticles and compare to a silicon standard. TEM imaging enables an estimate to be made of the size and shape of the nanocrystals synthesized. Finally EDS spectroscopy gives information on the elemental composition of our particles. These techniques allow us to prove the pure silicon nature of the quantum dots.

The crystalline nature of the nanocrystals can be assessed using X-ray diffraction spectroscopy (figure 3.3). The pattern is compared to the JCPDS card 27-1402 used as a standard for silicon crystalline structures. 


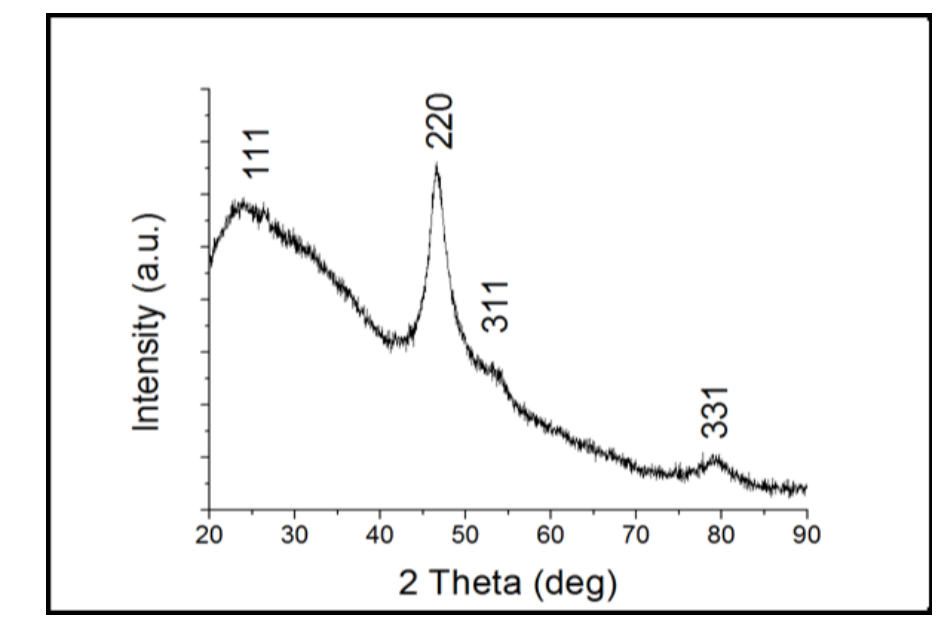

\begin{tabular}{|c|c|c|}
\hline Reflection & $\begin{array}{c}2 \text { Theta (deg) } \\
\text { measured }\end{array}$ & $\begin{array}{c}\text { 2 Theta (deg) } \\
\text { JCPDS card }\end{array}$ \\
\hline 111 & 25.31 & 28.44 \\
\hline 220 & 46.54 & 47.30 \\
\hline 311 & 54.02 & 56.12 \\
\hline 331 & 79.45 & 76.38 \\
\hline
\end{tabular}

Figure 3.3: XRD spectrum of silicon quantum dots and the comparison of the peak position with JCPDS card 27-1402

The diffraction peaks measured for the silicon nanocrystals sample we prepared using sodium borohydride are in good concordance with the reflection reported for the powder diffraction of silicon in the JCPDS card and confirm the diamond cubic structure. The four major diffraction peaks of silicon crystalline structures are observed and correspond to the scattering of the (111), (220), (311) and (331) planes. The slight shift towards smaller 2 theta values observed for our sample can be explained by the uniform strain that occurs in nanomaterials. The broadening of the peaks is explained by the non-uniform strain of the crystal lattice induced by the small size of the nanocrystals. ${ }^{20}$ The XRD pattern obtained here is consistent with silicon nanostructures distorted due to their small size. The peak broadening can also be explained by a less precise channelling of the diffracted rays, otherwise described as less reflections meeting the Bragg condition. 
The size and shape of silicon nanocrystals is assessed using the transmission electron microscope. Figure 3.4 pictures a representative image of silicon quantum dots. Using the variation of contrast between silicon quantum dots and the carbon-coated copper support grid, the shape can be determined and the size is evaluated by statistically averaging the measured diameter of the particles. Figure 3.4 represents nanoparticles synthesized using lithium borohydride as a reducing agent and allylamine as capping agent. The average size of the nanocrystals is estimated at $3.2 \pm 1.6 \mathrm{~nm}$.

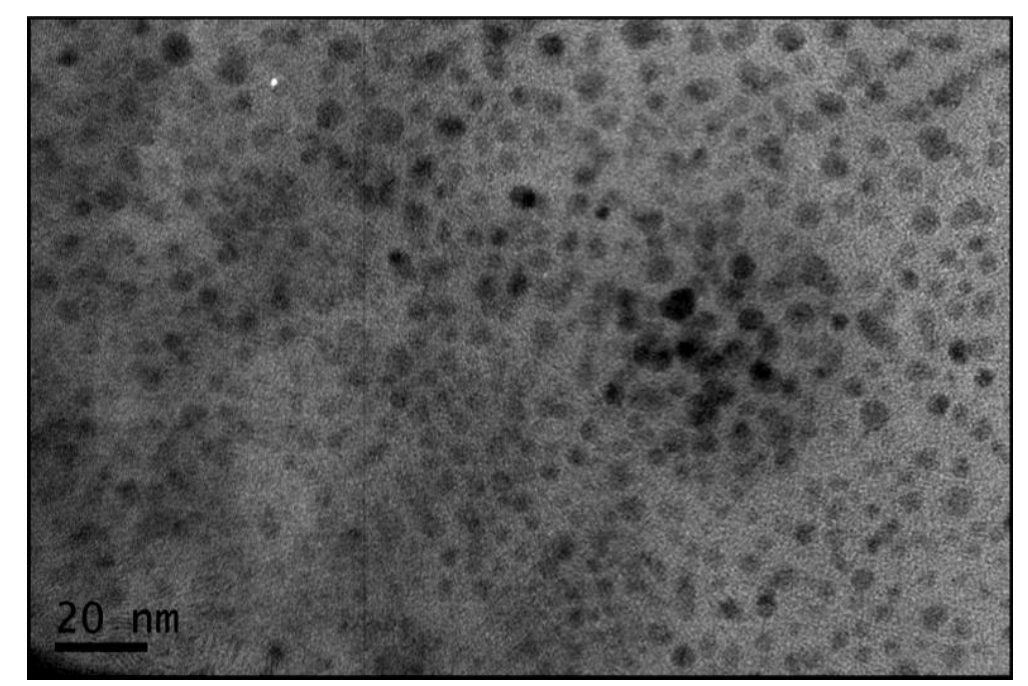

Figure 3.4: a TEM image of silicon quantum dots

Using the same sample support as for the TEM analysis, EDS spectroscopy can be performed. Figure 5 is the energy dispersive X-ray spectroscopy (EDS) analysis the silicon nanocrystals. EDS measures the X-ray produced by a sample under a beam of electrons. The EDS detector is associated to the transmission electron microscope. As the X-rays produced are characteristic to each element, EDS proves the pure silicon nature of the nanocrystals. 


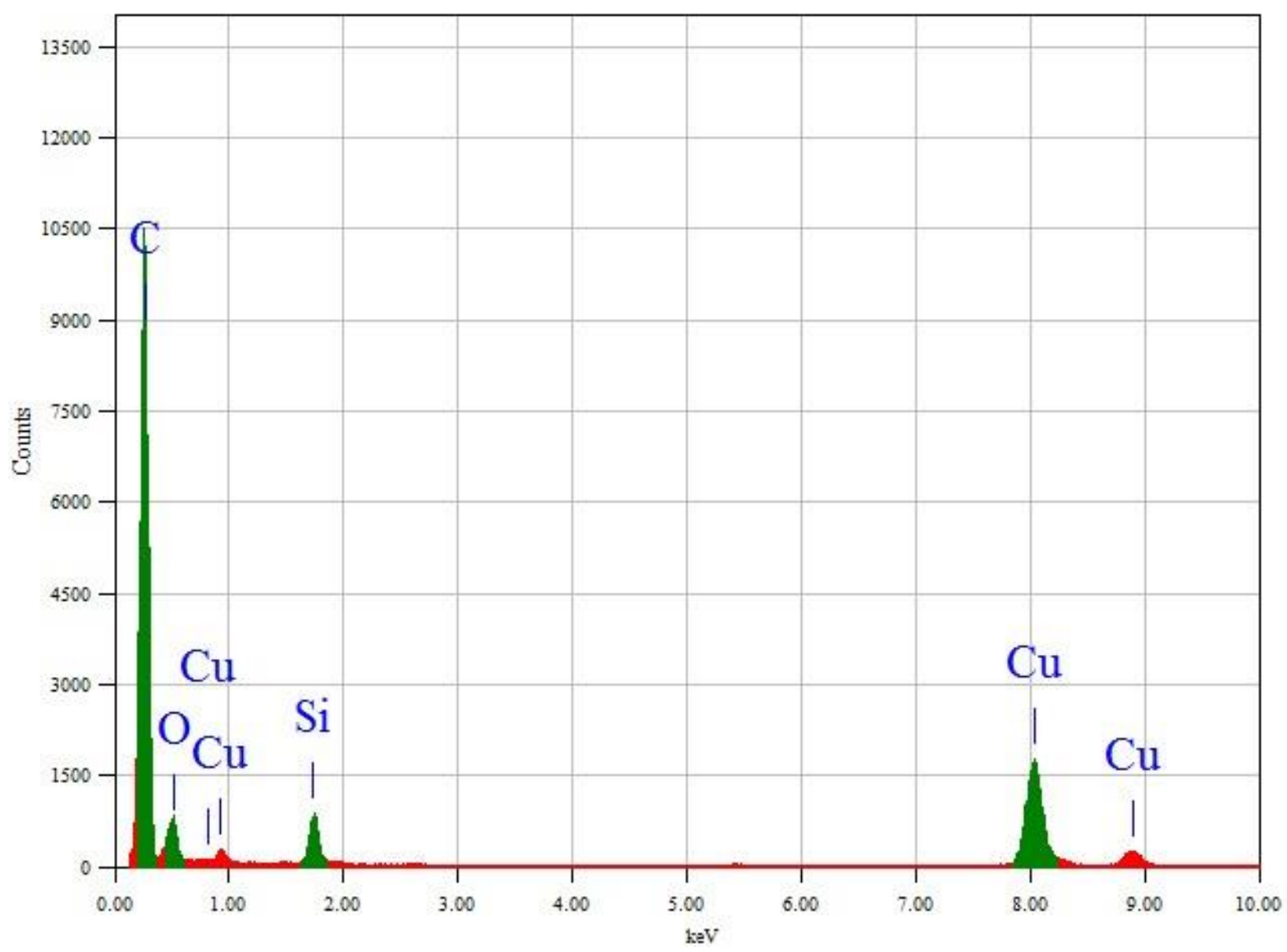

Figure 3.5: EDS spectrum of silicon nanocrystals on a carbon coated copper grid

The sharp peak at $1.75 \mathrm{keV}$ is associated with the silicon element, giving confirmation that the nanocrystals observed are composed of silicon. The peaks allocated to copper and carbon are due to the TEM grid, which was used as a support for the nanocrystals.

$X R D, T E M$ and EDS demonstrate the pure silicon nature of our quantum dots. The optical properties are then to be assessed. The next section will offer an elucidation to the physical and optical of silicon quantum dots synthesized using the reverse micelles synthesis protocol. 


\subsection{Optical properties of silicon quantum dots and their origin}

In its bulk form, silicon presents an indirect band gap and, consequently, no visible fluorescence. Silicon nanostructures have been reported to emit light in various wavelength regions, principally either red or blue luminescence. ${ }^{21-25}$ Following our synthetic method, a silicon quantum dots solution has a slight yellow colour and it displays a bright blue photoluminescence under excitation to UV light, as it is pictured in figure 6 .

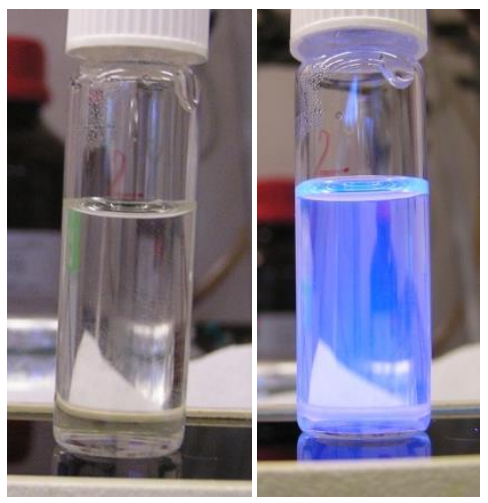

Figure 3.6: allylamine-capped silicon quantum dots in water under ambient light on the left and under UV light on the right

Standard measurements to evaluate the optical properties of silicon quantum dots consist of first absorption spectroscopy followed by photoluminescence spectroscopy. With absorption measurements, we want to quantify the energy required to excite an electron from Valence to conduction band in our quantum dots and speculate on the electronic transitions occurring. The absorption wavelength range also defines the spectral region for future fluorescence measurements. As many compounds and solvents absorb light in the UV region, a variation of concentration of silicon quantum dots in solution is expected to give confirmation that the absorption observed originates from the silicon quantum dots. Figure 7 represents the UV-Visible absorption dependence upon concentration of silicon quantum dots capped with allylamine in water. 


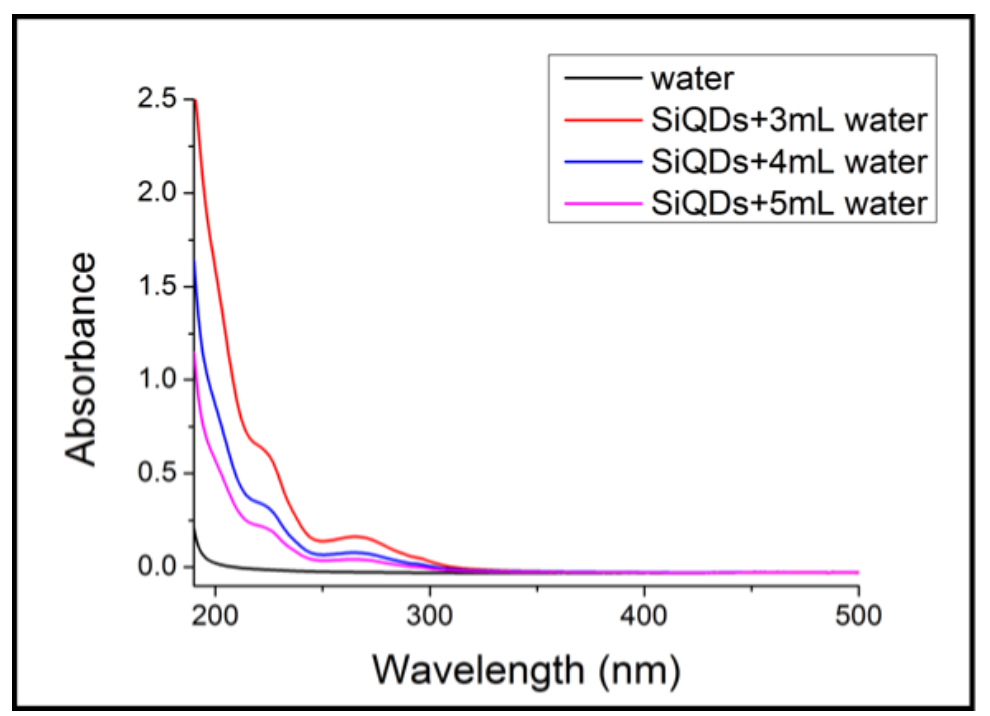

Figure 3.7: concentration dependence of the UV-Vis absorption for silicon quantum dots capped with allylamine

As it can be seen in figure 7, the silicon quantum dots dispersed in water exhibit absorption over the $190-320 \mathrm{~nm}$ range. Two shoulders are observed at 220 and $265 \mathrm{~nm}$. The absorption edge is around $335 \mathrm{~nm}$, corresponding to a band gap of $3.7 \mathrm{eV}$, attributed to a $\Gamma-\Gamma$ direct band gap transition. The positions of the peaks and the band gap are in good concordance with previous reports of silicon nanocrystals absorption. ${ }^{23,24}$ The absorbance decreases with decreasing concentration, from 0.6 to 0.2 on the first shoulder peak and from 0.2 to 0.07 for the second peak. The variation of absorbance for each maximum corresponds to the variation of concentration between the samples, proving that the portion of light is absorbed by the silicon quantum dots in solution.

We evaluate the light emission of our samples using a spectrofluorometer. The photoluminescence of the silicon quantum dots capped with allylamine in hexane is represented in figure 3.8. The spectra for our silicon nanocrystals clearly show an emission process. PL spectra were recorded between $280 \mathrm{~nm}$ and $360 \mathrm{~nm}$ with $20 \mathrm{~nm}$ intervals. The region of wavelength was chosen according to the absorbed wavelength (figure 3.7). The photoluminescence excitation experiment (figure 3.8) measures what excitation wavelength gives rise to what emission wavelength to confirm the origin of the photoluminescence. 


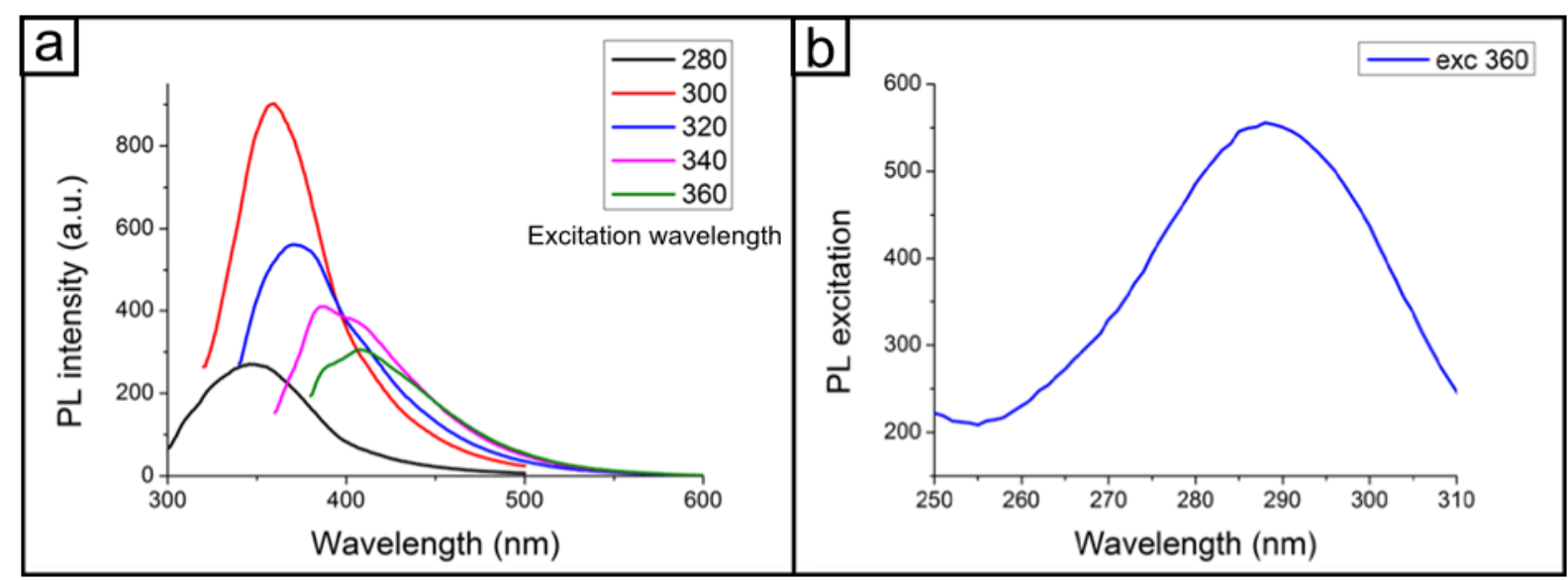

Figure 3.8: a) photoluminescence spectra of silicon quantum dots capped with allylamine dispersed in methanol and b) the photoluminescence excitation spectrum of the same quantum dots in methanol solution.

The silicon quantum dots solution show fluorescence between 300 and $550 \mathrm{~nm}$. The maximum emission for all the spectra shifts to higher wavelength with an increasing excitation wavelength. This shift can be explained by a slight variation of size of the nanocrystals in the sample, resulting in a variation of the bandgap due to quantum confinement. ${ }^{26}$ The emission measured for an excitation at $340 \mathrm{~nm}$ shows two separate emission maxima revealing two emission phenomena. The second peak could be explained by a surface or environmental effect. The maximum photoluminescence intensity is observed at $360 \mathrm{~nm}$ for an excitation wavelength of $300 \mathrm{~nm}$. Figure $3.8 \mathrm{~b}$ ) represents the photoluminescence excitation spectrum. As the photoluminescence emission peak was observed at $360 \mathrm{~nm}$ for an excitation wavelength of 300 , the photoluminescence excitation wavelength was set at $360 \mathrm{~nm}$. A PLE peak at $290 \mathrm{~nm}$ is observed for the excitation wavelength of $360 \mathrm{~nm}$. It confirms that the photoluminescence observed at $360 \mathrm{~nm}$ is only due to the silicon quantum dots excited by a $290 \mathrm{~nm}$ radiation.

This emission phenomenon observed for silicon quantum dots can be first attributed to quantum effects occurring in such small crystals. Several reports attribute the photoluminescence of silicon nanocrystals to a pseudo-direct band-gap transition, associated to the reciprocal space transition labelled $\Gamma-\Gamma$ or more generally to a localization of electrons in small crystals. ${ }^{26-29}$ This quantum confinement theory may explain what is occurring for the silicon quantum dots formed via reverse micelle 
synthesis. In order to conclude to that theory, a qualitative evaluation of surface chemistry and a quantification of the rate of the transition, via the measurement of the PL dynamic decay of the photoluminescence are necessary.

\section{Solvatochromism}

Two types of photoluminescence are usually observed for silicon quantum dots: red or blue emission. The blue emission is associated to a fast PL decay associated with a radiative or direct electron recombination. The red emission presents a slow dynamic decay, which is congruent with the photoluminescence originating from defects. To determine the origin of the emission process of our silicon quantum dots we first evaluate the influence of the surface chemistry by studying the behaviour of silicon quantum dots in different solvent environments. This study was part of the research conducted in collaboration between the Veinot, Kauzlarich and Tilley groups aimed to understand the origin of the red or blue luminescence observed for silicon quantum dots obtained by various methods. ${ }^{25}$ Figure 3.9 represents the normalized intensity of the photoluminescence of dodecyl-terminated silicon quantum dots in six different solvents. The objective of this solvatochromism study was to observe the PL spectra maximum emission dependence upon the polarity of the suspending solvents. ${ }^{30}$

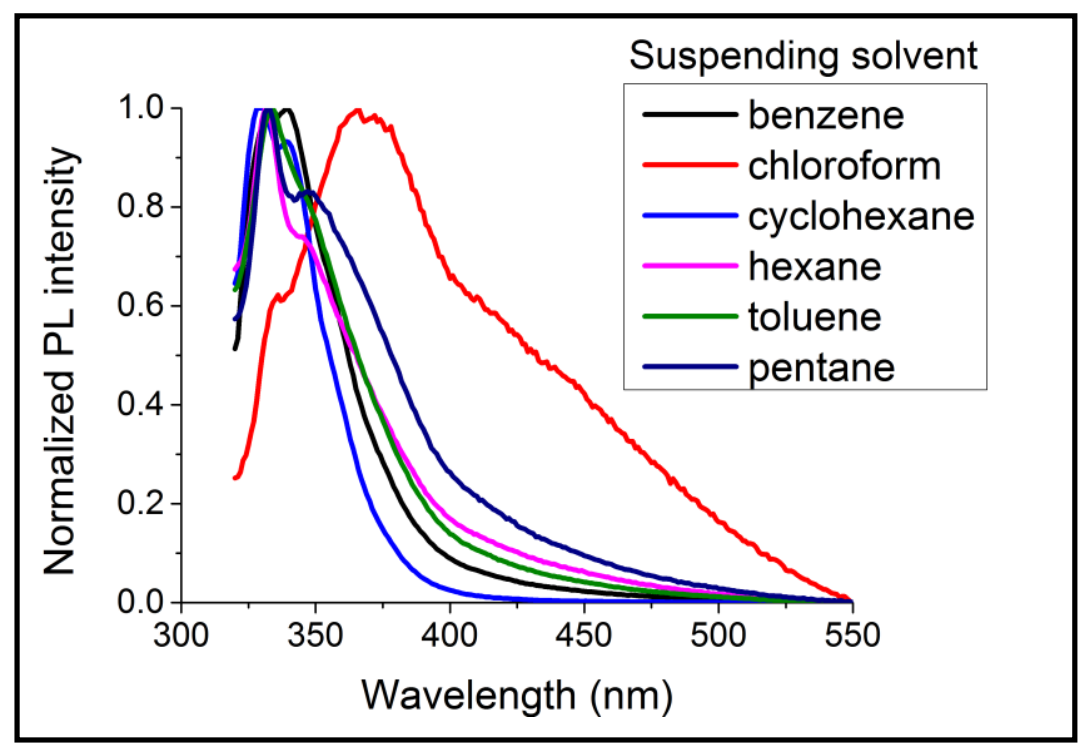

Figure 3.9: solvatochromism study for dodecene capped silicon quantum dots

The concentration of the silicon quantum dots in solution was kept constant with all the solvents. The excitation wavelength was set at $300 \mathrm{~nm}$. The intensity of the 
photoluminescence emission recorded was normalized to unity as it varied upon the solvent used. Benzene, hexane, cyclohexane, toluene and pentane have low polarities. Chloroform has a dielectric constant of 4.1 , which is medium value of polarity. With low polarity solvents, the maximum photoluminescence emission is situated between 330 and $345 \mathrm{~nm}$. When dispersed in chloroform, the silicon quantum dots emit light at 378 $\mathrm{nm}$, evidencing a red shift of the PL upon changes from low polarity to high polarity solvents. This shift indicates a coulombic interaction between silicon quantum dots and the solvent environment. A similar red shift of the photoluminescence upon increasing polarity of solvent has been observed for CdSe quantum dots by Leatherdale and Bawendi. ${ }^{31}$ Dasog et al conclude that the blue emission red shift with increasing polarity is consistent with the presence of nitrogen and oxygen as defects or surface atoms of silicon quantum dots. ${ }^{25}$ They based their theory on the similar blue photoluminescence observed for silicon oxynitride ${ }^{32-34}$ and its possible origin as a charge transfer between silicon and oxygen and nitrogen. ${ }^{35}$ On the other hand, the luminescence of silicon carbide nanoparticles has been reported to be located in the same wavelength region than silicon quantum dots by Reipa et al. ${ }^{36}$ This implies that the solvatochromism observed can also be an evidence of a silicon - carbon charge transfer. For the nanoparticles synthesized in this study, the surface interaction generating the red shift of the photoluminescence can originate from either a Si-C bond, or an occasional Si-N bond or a dipolar interaction with the solvent. Photoluminescence lifetime spectroscopy would help to determine the rate of recombination of the excitons and thus leading to a more defined answer to the origin of the photoluminescence of silicon quantum dots.

\section{Photoluminescence lifetime}

The solvatochromism study is evidence of a charge transfer if it is accompanied with a proof of short-lived excited state. Time-resolved photoluminescence measured the time in which the electrons recombined with a hole in the lower energy state after excitation. $^{28,37,38}$ The photoluminescence decay of our quantum dots in presented in figure 3.10. This study has been realised in collaboration with Boqian Yang from Michael Barnes's group at the University of Massachusetts-Amherst, USA. 

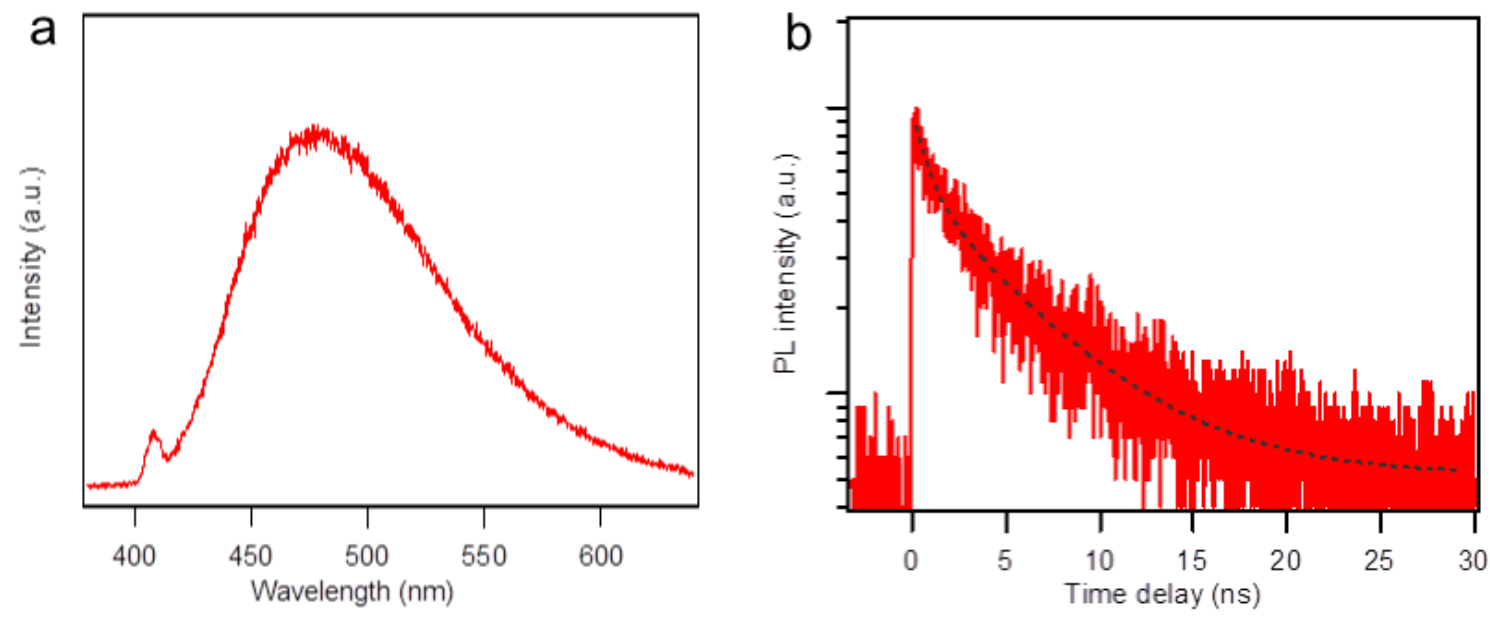

Figure 3.10: a) PL emission for a $405 \mathrm{~nm}$ excitation wavelength of silicon quantum dots in water and b) represents the time-resolved photoluminescence corresponding.

Figure 3.10 a) represents the photoluminescence of the allylamine-terminated silicon quantum dots solution in water at a $405 \mathrm{~nm}$ excitation wavelength. On figure $3.10 \mathrm{~b}$ ) is represented the PL lifetime corresponding to the emission recorded in figure $10 \mathrm{a}$ ). PL decay dynamics were taken at the peak emission wavelength $(478 \mathrm{~nm})$ with a band width of $5 \mathrm{~nm}$. Using the following exponential decay function,

$$
I(t)=I_{o} \exp (-t / \tau)^{b}
$$

Where $\tau$ is the characteristic PL lifetime and $\beta$ is the dispersion exponent used to approximate the quantum dot behaviour with a single exponential equation, the $\mathrm{PL}$ decay of silicon quantum dots in solution can be determined. The decay lifetime is determined to be $5.42 \mathrm{~ns}$. The PL decay lifetime suggests that the radiative decay is driven by a direct electron - hole recombination. The recombination time observed for indirect band gap material, such as bulk silicon is usually in the order of microseconds or even milliseconds. Direct band gap transitions are usually correlated with fast recombination processes associated with PL lifetimes in the 1 to 10 ns range. ${ }^{38}$ Silicon carbide nanostructures are also reported to present a PL decay in the order of 1-10 ns. ${ }^{36}$ Regarding the 5.42 ns PL lifetime exhibited by our sample, we can then assume that the photoluminescence observed for our silicon quantum dots is due to an electron transfer 
associated with a silicon - carbon transition between the silicon core and the capping molecule through the carbon bond or to a transition of pseudo-direct band gap transition labelled $\Gamma-\Gamma($ see figure 1.4$){ }^{2}$ However, the short dynamic decay eliminates the possibility of the PL originating in a defect driven emission. ${ }^{38}$

The direct band gap property of quantum confined silicon nanocrystals can be deduced from a study of the optical properties upon a variation of the mean size of the nanocrystals. This variation of size can be achieved by using different reducing agents during the synthetic process.

\subsection{Particle size effect on the optical properties of silicon quantum dots}

In this section, we are interested in the influence of the size of the silicon nanocrystals on their optical properties. The changes in the optical properties of silicon quantum dots with particle size is expected to provide an insight on the electronic transitions occurring and evidence probable quantum confinement effects in silicon nanostructures. Those effects are expected to be similar to direct band gap materials, such as $\mathrm{Cd}-\mathrm{X}$ or $\mathrm{Pb}-\mathrm{X}$ materials $\left(X=S\right.$ or Se), exhibiting a variation of PL emission upon nanoparticle size. ${ }^{39} \mathrm{We}$ propose to produce a size variation of silicon quantum dots by using a variety of hydride reducing agents. The strength of reducing agent influences the speed of decomposition of the metal precursor. According to the research conducted by Amane Shiohara and Sujay Prabakar, the reducing agent is the main factor controlling the size of silicon nanocrystals. ${ }^{3}$ This study compares three different reducing agents and their influence on the size of the silicon quantum dots and also on their optical properties.

In these experiments, silicon tetrachloride was reduced by sodium borohydride, lithium borohydride or lithium aluminium hydride. Sodium borohydride is the weakest reducing agent used in this study, while lithium aluminium hydride is the strongest. In all three experiments, the nanoparticles were capped with allylamine after particle growth. The allylamine is expected to covalently bond to the silicon nanocrystal through the double bond. The amine terminated silicon quantum dots are water soluble. These experiments followed and developped the work of Sujay Prabakar, who evaluated the effects of 
reducing agents on alkyl-terminated silicon quantum dots, synthesized in a smaller scale. The synthetic route used for the silicon nanocrystals reported here is a scale-up of the experimental procedure followed by Dr.Prabakar as we employed a solution more than 3 times more concentrated in silicon precursor.

After 3 cycles of size exclusion column chromatography, the structural nature of the synthesized nanocrystals is assessed by transmission electron microscopy. Figure 3.11 represents TEM images for the samples synthesized using the 3 different reducing agents and their corresponding size distribution. 


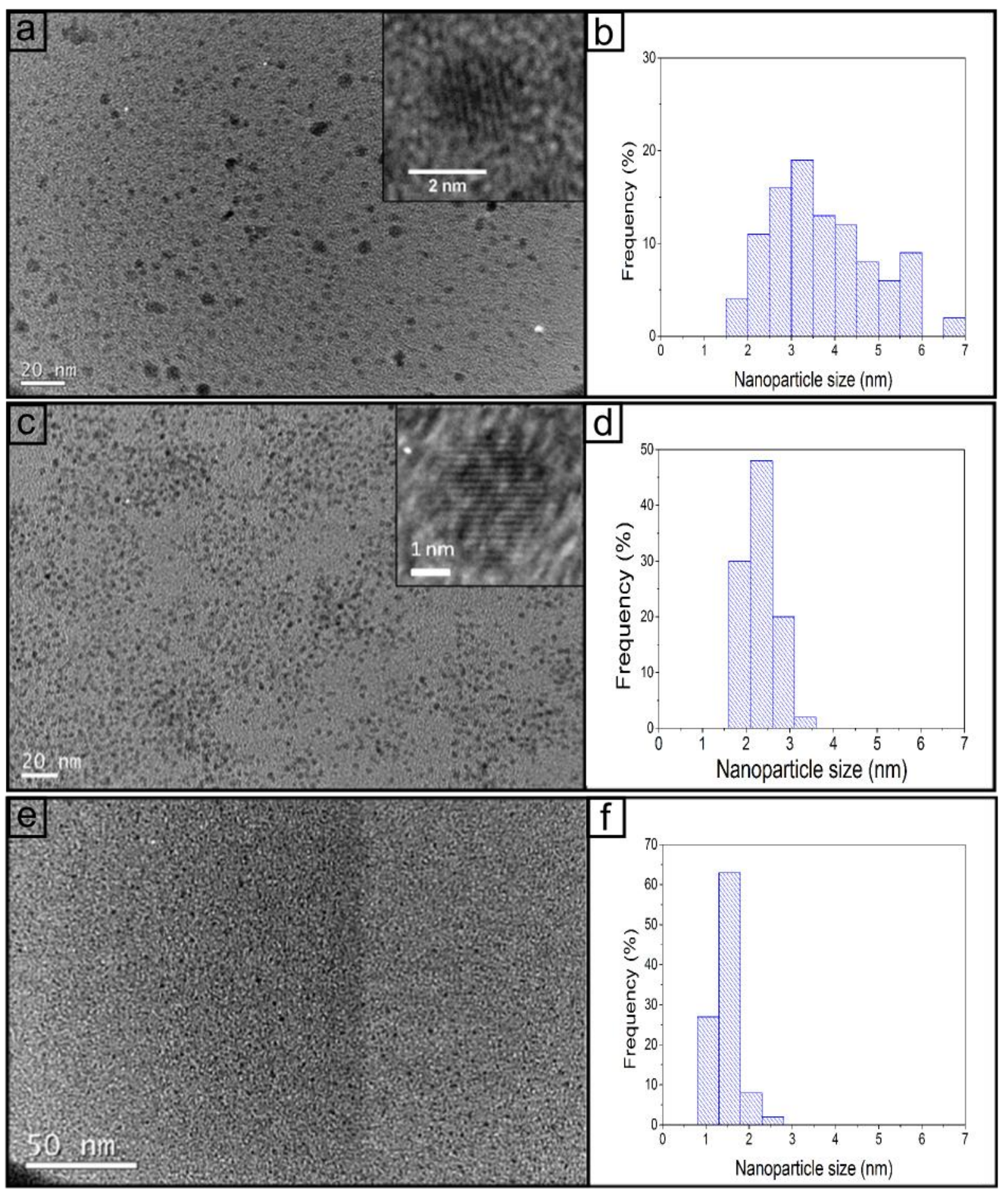

Figure 3.11: Low resolution TEM image of the amine terminated-silicon nanocrystals obtained by the reduction of $\mathrm{SiCl}_{4}$ by a) $\mathrm{NaBH}_{4}$, c) $\mathrm{LiBH}_{4}$ and e) $\mathrm{LiAlH}_{4}$. The diagrams b), d) and f) represent the corresponding size distribution diagram of the a), c) and e) images respectively. The insets in a) and c) are high resolution TEM images of two silicon nanocrystals, corresponding to the a) and c) samples. 
The nanoparticles have a spherical shape for the samples obtained with all three reducing agents. Using sodium borohydride, the nanocrystals range from 1.5 to $7 \mathrm{~nm}$ with an average size of $3.7 \pm 2.6 \mathrm{~nm}$. For nanoparticles obtained by chemical reduction with lithium borohydride, the average size of the nanocrystals is estimated at $2.3 \pm 0.7$ $\mathrm{nm}$ from the average of measuring over 100 nanocrystals. Finally, using lithium aluminium hydride as reducing agent, the size of the silicon quantum dots is estimated to be $1.5 \pm 0.7 \mathrm{~nm}$. The particle size is shown to be significantly reduced with increasing strength of reducing agent. This observation is in good concordance with the LaMer model, which states that a shorter nucleation event, or a 'burst' nucleation, lead to a more homogeneous size and concomitantly smaller size distribution. ${ }^{40}$

The insets of figure 3.11 a) and c) represent high resolution TEM images of two nanocrystals obtained using $\mathrm{NaBH}_{4}$ and $\mathrm{LiBH}_{4}$ respectively as reducing agents. Both images show one set of lattice fringes per nanocrystal. This attests to the monocrystalline nature of the nanoparticles. The distance between fringes is measured at $2.1 \AA$ and $2.0 \AA$ respectively, which is characteristic of $\mathrm{Si}\{220\}$ lattice spacing.

The UV-Vis absorbance spectra of the nanocrystals in solution for the samples obtained for the three different reducing agents are represented in figure 3.12. The nanocrystals were dispersed in methanol and homogenized for 2 minutes in an ultrasonic bath prior to the measurement. 


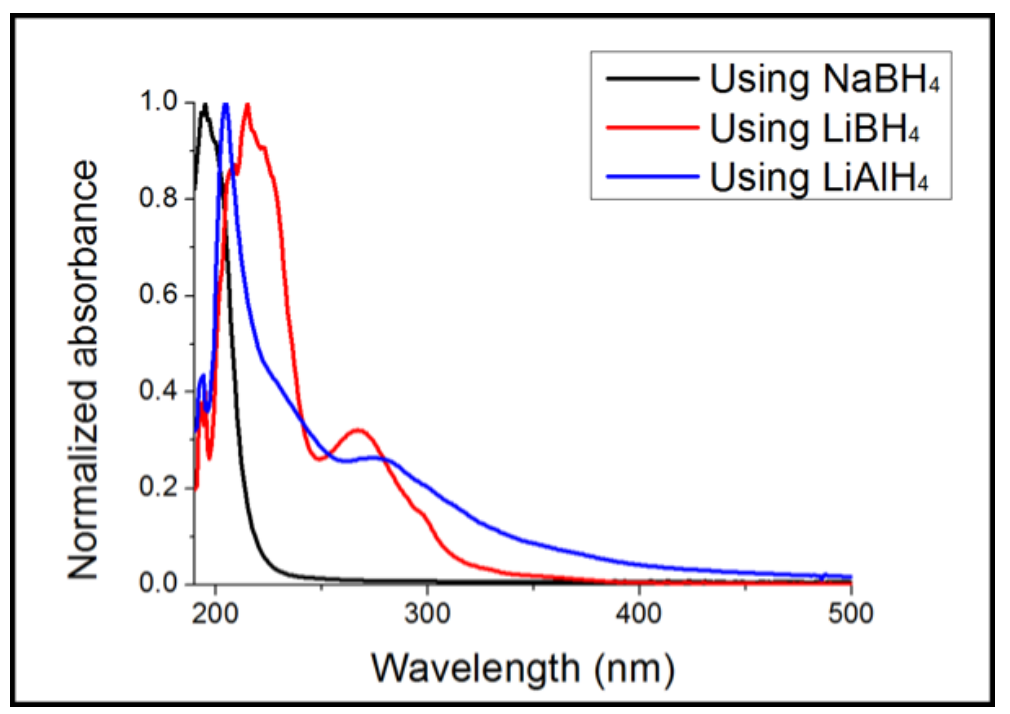

Figure 3.12: comparison of normalized UV-Vis absorption spectra of silicon QDs synthesized using $\mathrm{NaBH}_{4}(3.7 \mathrm{~nm}), \mathrm{LiBH}_{4}(2.3 \mathrm{~nm})$ and $\mathrm{LiAlH}_{4}(1.5 \mathrm{~nm})$ and capped with allylamine

For the nanocrystals synthesized using sodium borohydride suspended in methanol, only one UV-Visible spectral absorption peak is observed at $198 \mathrm{~nm}$, which corresponds to a single electronic transition occurring in the nanocrystals. Methanol solution of samples synthesized using $\mathrm{LiBH}_{4}$ and $\mathrm{LiAlH}_{4}$ present two absorption peaks, at 206 and 285 and a shoulder at $240 \mathrm{~nm}$ for $\mathrm{LiAlH}_{4}$ and peaks at 213 and $265 \mathrm{~nm}$ and a shoulder at $300 \mathrm{~nm}$ for $\mathrm{LiBH}_{4}$. As the second peak and the shoulder are present only for smaller particles, we can attribute these events to a transition associated with quantum confinement effects. The blue shift of this absorption edge with decreasing size, as well as the increase in wavelength of the peak position are in good concordance with the theory of the pseudo-direct band gap transition previously reported for quantum confined silicon quantum dots. The increase of the absorption edge is consistent with the increase in band gap energy with decreasing size, observed in other direct band gap quantum dots, such as CdSe. ${ }^{41}$

After a comparison of the absorption properties of the silicon quantum dots of three different sizes, we are interested in the emission process occurring in the same samples. For the sample synthesized using $\mathrm{NaBH}_{4}$, the exhibited photoluminescence exposed to a UV-lamp at $365 \mathrm{~nm}$ is weak. The samples synthesized using $\mathrm{LiBH}_{4}$ and $\mathrm{LiAlH}_{4}$ display a stronger blue photoluminescence on exposure to the UV-Lamp. The PL emission spectra recorded with a spectrofluorometer are represented in figure 3.13. 


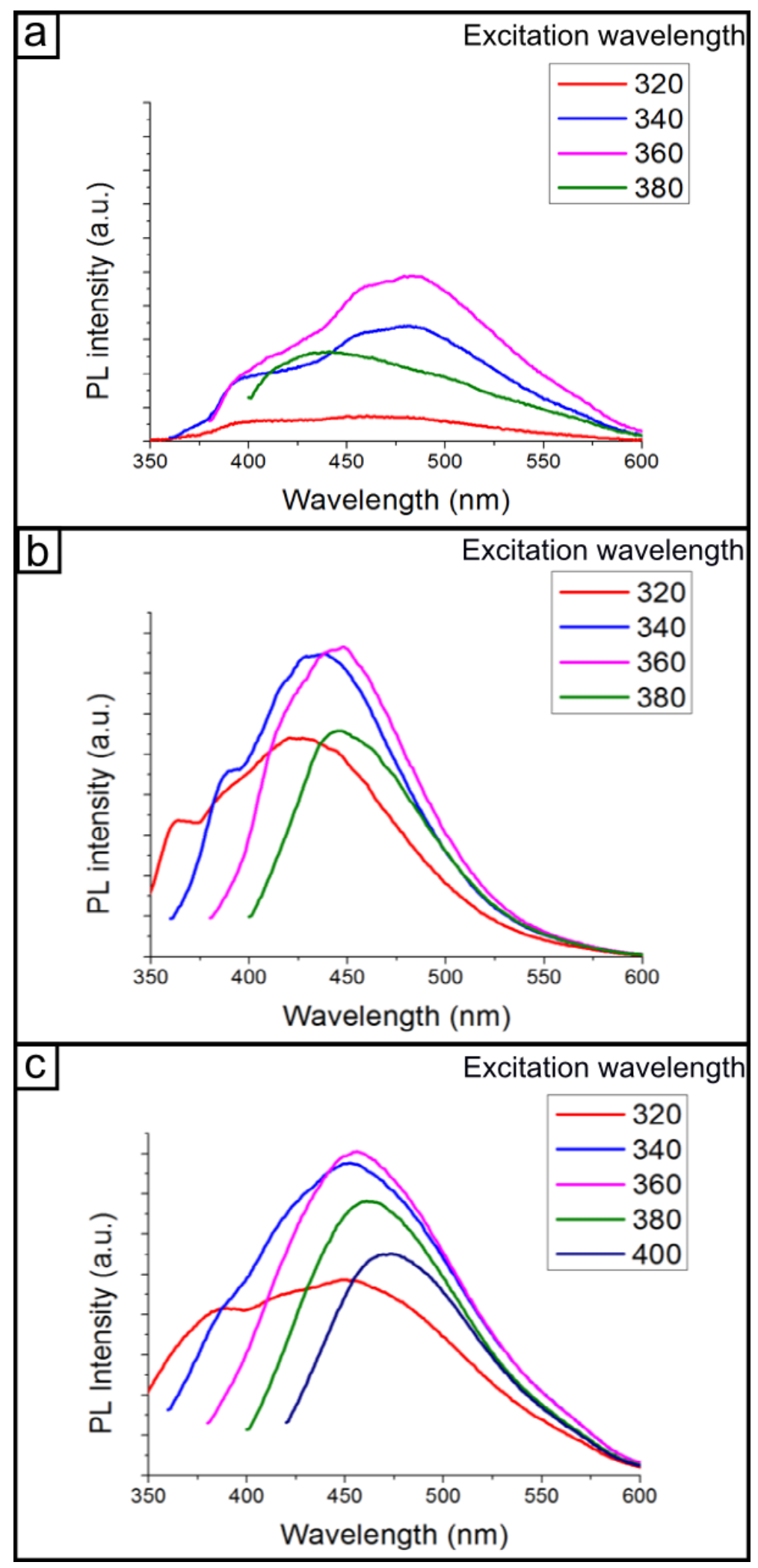

Figure 3.13 : excitation wavelength-dependent photoluminence silicon quantum dots with an allylamine surface passivation reduced with a) $\mathrm{NaBH}_{4}$, b) $\mathrm{LiBH}_{4}$ and c) $\mathrm{LiAlH}_{4}$. 
The PL spectra were recorded for excitation wavelength between $320 \mathrm{~nm}$ and 380 or $400 \mathrm{~nm}$ of with $20 \mathrm{~nm}$ intervals. A similar trend is observed for all the three samples. The photoluminescence peaks present a red shift with increasing excitation wavelength. For the silicon nanocrystals synthesized by the mean of $\mathrm{NaBH}_{4}$, the recorded PL intensity using the fluorometer is also weak, compared to the ones synthesized using lithium based reducing agents. This observation is evidence of a quantum confinement phenomenon, as the nanocrystals with a size below the Bohr radius of silicon exhibit a stronger photoluminescence. The weak $\mathrm{PL}$ of the $\mathrm{NaBH}_{4}$ reduced sample is then explained by a mean size larger than the $4.3 \mathrm{~nm}$ Bohr radius, with a few smaller nanocrystals.

The experiments were successful in producing silicon quantum dots using $\mathrm{NaBH}_{4}, \mathrm{LiBH}_{4}$ and $\mathrm{LiAlH}_{4}$ as a reducing agent. The particles size distribution remains relatively wide for the particles using sodium based reducing agent. TEM images and optical properties are in good concordance with previous reports by Prabakar et al and Kauzlarich et al showing that the different reactivities of reducing agents can result in a control of the particle size. ${ }^{3,42,43}$ The next experiments would report on the effect of the surface chemistry of the silicon nanocrystals.

\subsection{Surface effects on silicon quantum dots}

The chemical reduction of silicon tetrachloride results in hydride terminated silicon quantum dots via the reverse micelle synthesis process. The surface is then modified through a UV-induced hydrosilylation using various molecules. Silicon nanocrystals can be capped with either non-polar molecules, such as 1-dodecene or 1-hexene or polar molecules, such as allylamine. The main interest of using polar or non-polar surface termination is the solubility in convenient solvents. Using alkene molecules results in alkyl-terminated nanocrystals that are soluble in non-polar solvents. In contrast, allylamine-capped silicon quantum dots would be soluble in polar solvents, such as water, a property essential for biological applications. While the surface chemistry of the 
silicon quantum dots is not expected to provide structural difference on the nanocrystals, the surface molecule is predicted to affect the optical properties. In this study, we compare silicon quantum dots synthesized following exactly the same growth process using lithium borohydride to reduce silicon tetrachloride with three different capping molecules: allylamine, 1-hexene and 1-dodecene and TOAB as a surfactant.

\subsubsection{Structural characterization}

The structural nature of the synthesized nanocrystals is assessed by transmission electron microscopy. Figure 3.14 represents TEM images for the samples synthesized using the 3 different surface passivation molecules and their corresponding size distribution. The nanoparticles have a spherical shape for all three samples. For dodecene-capped silicon nanocrystals (figure 3.14.a), the average size was estimated at $2.5 \pm 0.9 \mathrm{~nm}$ by measuring over 100 nanoparticles. The average size of the allylamineterminated nanocrystals, represented in figure 3.14.c is estimated at $2.3 \pm 0.7 \mathrm{~nm}$. The low resolution TEM image in figure 14 shows the spherical shape of the nanocrystals. Finally, the 1-hexene passivated nanoparticles are averaged to be $2.6 \pm 1.1 \mathrm{~nm}$ in diameter. As it was expected, the surface passivation does not influence the size of the particles. The particles were formed before the UV-treatment and the hydrosilylation is a post-formation process, thus confirming that the shape and size of the silicon quantum dots is not influenced by the capping molecule.

The insets of figure 3.14 a) and c) represent high resolution TEM images of two nanocrystals obtained using 1-dodecene and allylamine respectively as surface capping molecules. Both images show one set of lattice fringes per nanocrystal. This attests of the monocrystalline nature of the nanoparticles. The distance between fringes is measured at $2.0 \AA$ for allylamine-terminated quantum dots (see inset in figure 3.14.c), which is characteristic of $\mathrm{Si}\{220\}$ lattice spacing, and fringes for the 1-dodecene terminated crystal are measured to be $3.1 \AA$ apart, which corresponds to the $\mathrm{Si}\{111\}$ lattice spacing. Low resolution and high resolution TEM images confirm that the surface chemistry applied to the silicon quantum dots after the growth process does not influence the physical shape of the nanocrystals. 

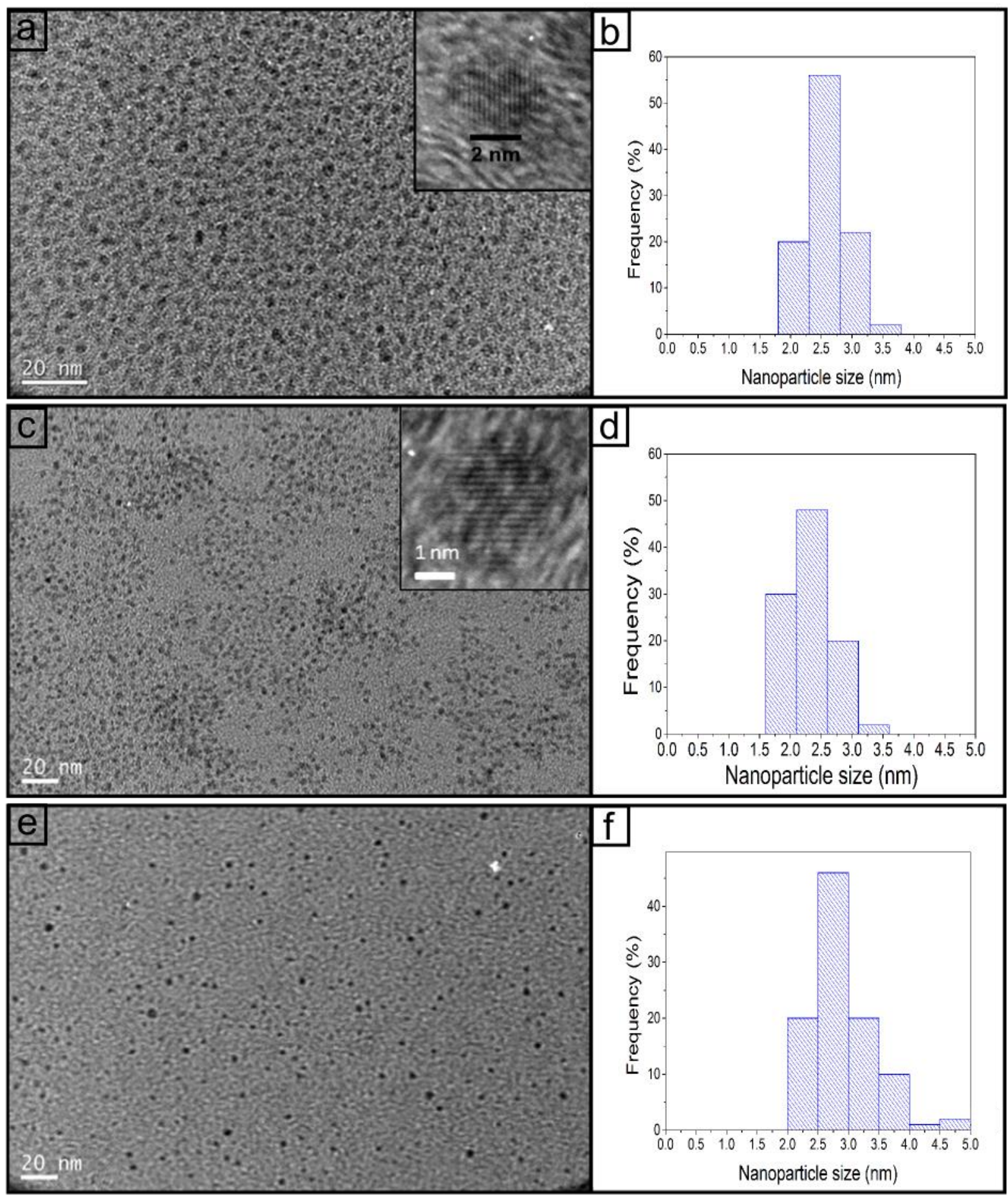

Figure 3.14: Low resolution TEM image of the silicon nanocrystals terminated by a) 1dodecene, c) allylamine and e) 1-hexene and their corresponding size distribution diagram in b), d) and f) respectively. Insets in a) and c) represent high resolution TEM images of one silicon nanocrystal with 1-dodecene and allylamine respectively. 
Using FTIR spectroscopy, we can confirm the surface passivation of our silicon quantum dots with information on the chemical bonds present in the sample by measuring the vibrations of the chemical bondings in the capping molecule under infrared wavelengths excitation. Figure 3.15 shows the Fourier transformed infrared spectrum obtained for allylamine, 1-hexene and 1dodecene-capped silicon quantum dots mixed into a $\mathrm{KBr}$ pellet. 


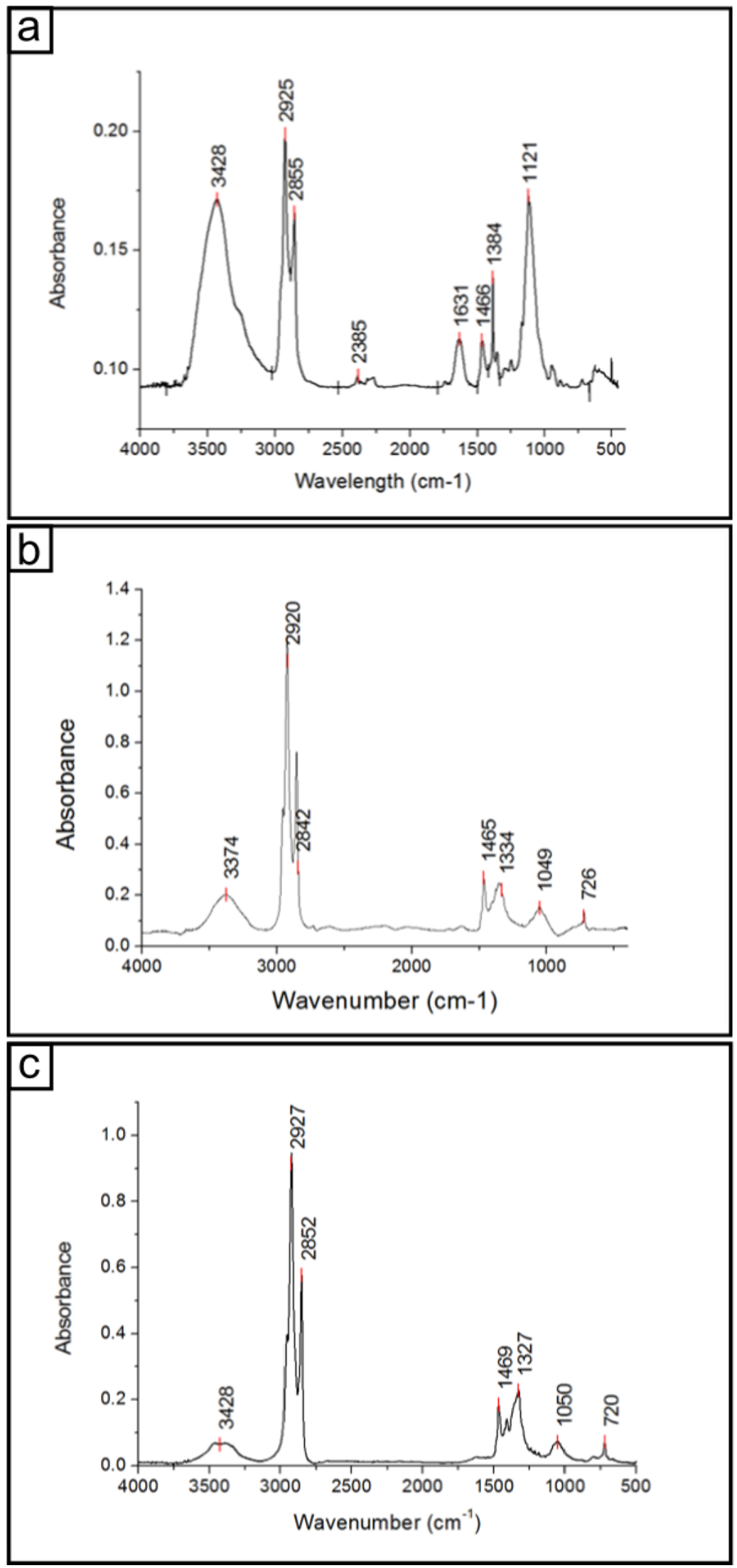

Figure 3.15: Fourier-transformed infrared spectroscopy spectra for silicon quantum dots capped with a) allylamine, b) 1-dodecene and c) 1-hexene. 
In figure 3.15 a) is represented the allylamine-terminated FTIR spectrum. The peaks at $1384 \mathrm{~cm}^{-1}$ and $1466 \mathrm{~cm}^{-1}$ correspond to the $\mathrm{Si}-\mathrm{CH}_{2}$ vibrational modes. The peak at 1121 $\mathrm{cm}^{-1}$ corresponds to the $\mathrm{C}-\mathrm{N}$ stretching from allylamine. The peak at $1631 \mathrm{~cm}^{-1}$ represents the amine bond. $\mathrm{N}-\mathrm{H}$ bond is also observed in the peak at $3428 \mathrm{~cm}^{-1}$. The peaks at $2855 \mathrm{~cm}^{-1}$ and $2925 \mathrm{~cm}^{-1}$ can be attributed to the symmetrical and asymmetrical stretching of $\mathrm{C}-\mathrm{CH}_{2}$. The peak at $2385 \mathrm{~cm}^{-1}$ presenting a low intensity is attributed to $\mathrm{Si}-\mathrm{N}$ or Si-O vibrations, representing a small quantity of surface defects. This FTIR spectrum indicates a successful attachment of allylamine molecules on silicon quantum dots through the breaking of the double bond in allylamine. As it can be seen on figure $15 \mathrm{~b}$ ) and c) are represented the FTIR spectra for the 1-dodecene and 1-hexene surface termination respectively, the peaks at $1334-1327 \mathrm{~cm}^{-1}$ and $1465-1469 \mathrm{~cm}^{-1}$ represent the $\mathrm{Si}-\mathrm{CH}_{2}$ vibration modes. The peaks at $2842-2852 \mathrm{~cm}^{-1}$ and $2920-2927 \mathrm{~cm}^{-1}$ can be attributed to the symmetrical and asymmetrical stretching of $\mathrm{C}-\mathrm{CH}_{2}$. These peaks assess the capping of silicon quantum dots with 1-dodecene/1-hexene. The peak at 3374 and $3428 \mathrm{~cm}^{-1}$ can correspond to occasional $\mathrm{Si}-\mathrm{O}$ bonds or $\mathrm{O}-\mathrm{H}$ bonds due to residual solvent in the sample. The peaks at $1049-1050 \mathrm{~cm}^{-1}$ and $726-720 \mathrm{~cm}^{-1}$ can be attributed to $=\mathrm{C}-\mathrm{H}$ bending, which can indicate the presence of a small quantity of free 1-dodecene or 1-hexene. Fourier-transformed infrared spectroscopy gives a good insight on the way the capping molecule is attached to the surface of the silicon quantum dots. However, solvent impurities and remaining small quantities of surfactant can contribute to the recorded signal and, due to similar chemical bondings, their signal cannot be readily separated. This leads to a nuclear magnetic resonance study of the silicon quantum dots solutions.

${ }^{1} \mathrm{H}$ NMR permits to distinguish the different environments of the protons and to conclude on the relative position of every hydrogen atom in the capping molecule. Figure 3.16 shows the ${ }^{1} \mathrm{H}$ NMR of the silicon quantum dots capped with allylamine (a) and 1-dodecene (b) in a solution of deuterated chloroform. 


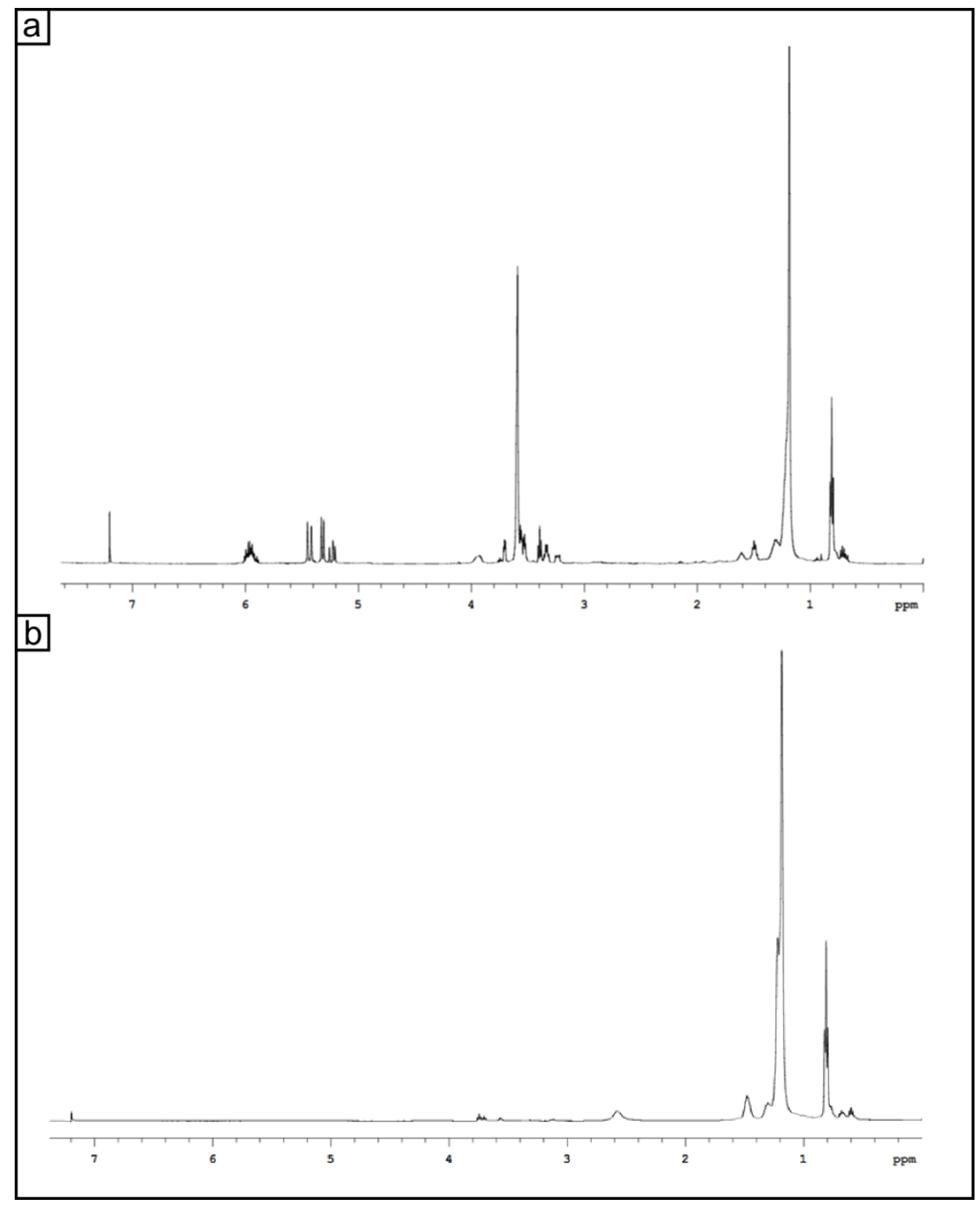

Figure 3.16: Nuclear magnetic resonance spectra for silicon quantum dots solutions in deuterated chloroform for a) allylamine capping and b) 1-dodecene surface termination. 
On the spectrum corresponding to allylamine-terminated silicon nanocrystals, at $\delta=$ $1.25 \mathrm{ppm}$ can be observed a peak corresponding to the protons of the amine group of allylamine $\left(\mathrm{N}-\mathrm{H}_{2}\right)$. The peak at $\delta=3.70 \mathrm{ppm}$ can be attributed to the protons in the carbon chain of allylamine neighbouring the amine group $\left(\mathrm{Si}-\mathrm{CH}_{2}-\mathrm{CH}_{2}-\mathrm{CH}_{2}-\mathrm{NH}_{2}\right)$. The triplet observed at $\delta=0.8$ represents the protons close to the silicon quantum dots ( $\mathrm{Si}$ $\mathrm{CH}_{2}-\mathrm{CH}_{2}-\mathrm{CH}_{2}-\mathrm{NH}_{2}$ ). The multiplet at about $\delta=6 \mathrm{ppm}$ and peaks from 5.2 to $5.5 \mathrm{ppm}$ correspond to the spectrum of free allylamine. As all the allylamine has been evaporated under vacuum, it can be concluded that for a minority of allylamine molecules, the amine group is attached to the surface of the silicon quantum dots. The relatively low intensity peaks between $\delta=3.2 \mathrm{ppm}$ and $3.6 \mathrm{ppm}$ show a remaining impurity of surfactant. NMR measurements confirm the allylamine attachment on the surface of the silicon quantum dots and the largely successful purification process as the impurities peaks are of relative low intensity. In figure $16 \mathrm{~b}$ ), from $\delta=1.1$ to $1.4 \mathrm{ppm}$ can be observed a peak corresponding to the protons of the carbon chain of 1-dodecene ($\mathrm{CH}_{2}-\mathrm{CH}_{2}-\mathrm{CH}_{2}-$ ). The triplet observed at $\delta=0.8$ represents the protons close to the silicon quantum dots $\left(\mathrm{Si}-\mathrm{CH}_{2}-\mathrm{CH}_{2}-\ldots-\mathrm{CH}_{2}-\mathrm{CH}_{3}\right)$. The absence of peak at $\delta=3.3 \mathrm{ppm}$ assessed the absence of surfactant impurity in the sample. These nuclear magnetic resonance for our samples rises to the deduction that the surface molecules allylamine and 1dodecene are attached to the surface of the silicon quantum dots, through the carbon double bond of the free molecules. This observation is concistent with the FTIR spectra of the silicon particles described above.

TEM images indicate that the silicon quantum dots are structurally equivalent. FTIR and NMR experiments give an insight on the manner the capping molecules are anchored to the surface of the silicon quantum dots. Studying the optical properties of those three different samples of silicon quantum dots will help to elucidate the role played by the surface chemistry on the physical properties. 


\subsubsection{Effect of surface capping on optical properties}

In this section the effect of surface passivation of the silicon quantum dots on the optical properties of the particles resulting from a variation of the possible electronic transitions between the silicon core and the outside molecule is sought.

All three samples of silicon quantum dots in a hexane solution were analysed using a UV-Visible absorption spectrometer. The UV-Visible absorption spectrum of silicon nanocrystal solutions are presented in figure 3.17.

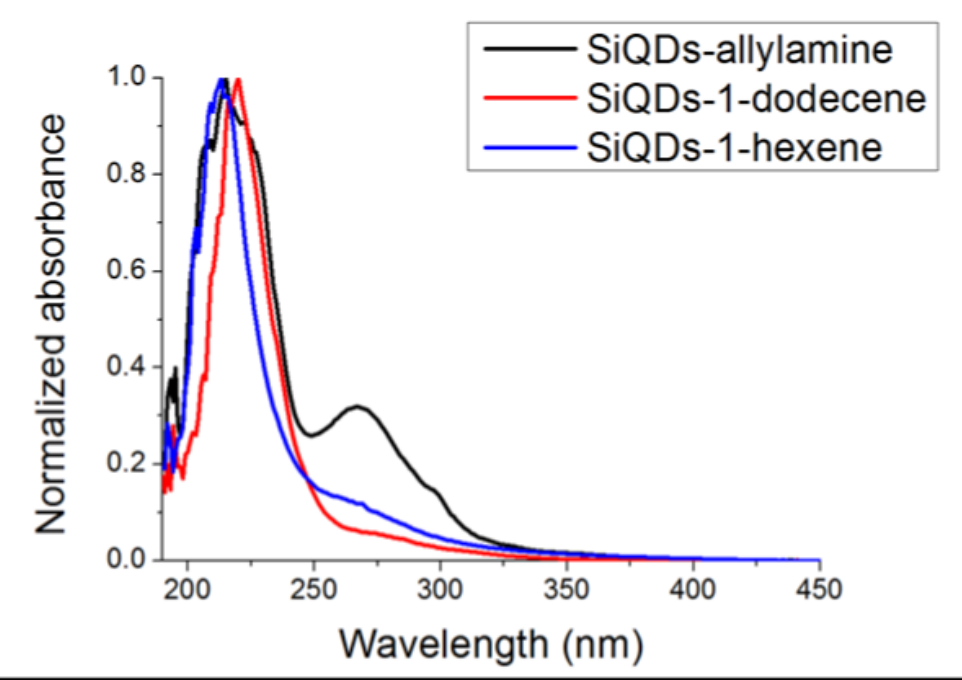

Figure 3.17: UV-Visible absorption spectrum for 1-dodecene capped silicon quantum dots.

All the samples present a major peak around $210 \mathrm{~nm}$. As the position of the peak does not vary according to the surface molecule, we can attribute the peak to a transition occurring in the core of silicon quantum dots. The same peak was for the study on the different reducing agents. A second peak is observed for those samples, with maxima around $265 \mathrm{~nm}$. The intensity of that peak varies between the different capping molecules. This second peak was attributed to a quantum confinement effect in the section 3.2.3. The observed absorption properties are in good agreement with previous reports of alkyl- and allylamine terminated quantum dots. ${ }^{2,3}$ The theory developed by the Kauzlarich group that a ligand-core transition is occurring would result in a shift of the absorption between different reducing agents and therefore does not apply to our 
observation. ${ }^{39}$ The variation of intensity can indicate an interaction between the capping molecule and the solvent.

Figure 3.18 represents the photoluminescence spectra recorded for the silicon quantum dots with 1-dodecene, 1-hexene and allylamine passivation solution in hexane. Each set of spectrum was recorded between 280 and $380 \mathrm{~nm}$ with $20 \mathrm{~nm}$ intervals. The same trend is observed for all the three surface molecules. A slight variation of maximum emission is observed, but it can also be attributed to the slight variation of nanoparticle size between the samples. Even if we expect the covalent bonds on the surface of the quantum dots to create localized defects and give rise to a new source of photoluminescence, it seems that the surface molecule does not influence dramatically the emission process. The alkyl or amine termination does not generate variation of the $\mathrm{PL}$, and neither does the length of the carbon chain. The influence of the solvent used generates a much more striking emission shift, as we described for the solvatochromism study in section 3.2.2. 


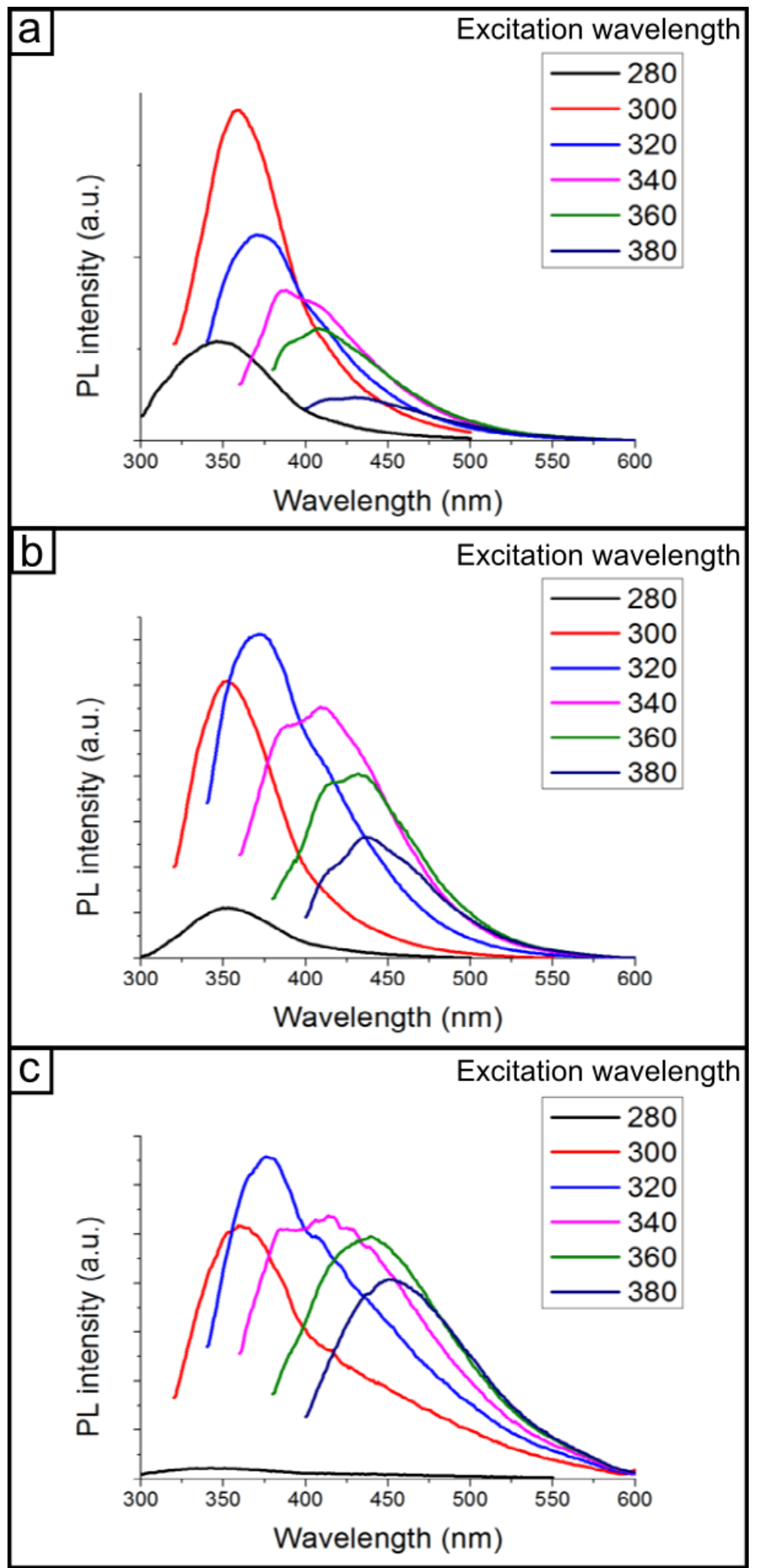

Figure 3.18: photoluminescence emission for excitation wavelength between 280 and 380 for different surface termination of silicon quantum dots, with a) 1-dodecene, b) allylamine and c) 1-hexene. The spectra were recorded for silicon quantum dots solution on hexane for the 3 specimen. 


\subsection{Discussion and summary}

\subsubsection{Origin of the photoluminescence of silicon quantum dots}

UV-Vis absorption spectroscopy concurs with previous reports of absorption for silicon quantum dots. The $210 \mathrm{~nm}$ peak is attributed to the transition expected for bulk silicon; the second peak is congruent with the theory of the $\Gamma-\Gamma$ pseudo-direct transition for quantum confined silicon nanocrystals. The photoluminescence measurements lead to the conclusion that the emission process can originate from the pseudo-direct band gap transition with some environmental effects such as a $\mathrm{Si}-\mathrm{C}$ or $\mathrm{Si}-\mathrm{N}$ bond on the surface or dipolar interaction with the dispersing solvent. The solvatochromism study showing a dependence of the photoluminescence upon the polarity of a solvent which indicates that the origin of the photoluminescence can be due to a charge transfer between silicon atoms and surrounding atoms when combined to a short-lived excited state. Veinot et al reported that the presence of nitrogen containing molecules on the surface of silicon quantum dots can induce a fast decaying blue emission. ${ }^{25}$ Reipa et al proved the blue fluorescence of silicon carbide nanocrystals. ${ }^{36}$ The time-resolved photoluminescence of our particles shows a fast transition (5 ns) corresponding to a charge transfer mechanism. The origin of the photoluminescence of the silicon quantum studied in this research is the possible pseudo-direct band gap transition due to the quantum confinement of silicon combined to a surface effect with charge transfers occurring between silicon and carbon and occasionally silicon and nitrogen or charge transfers between the solvent surrounding and the trap states on the surface of the quantum dots. More advanced spectroscopy such as ultrafast laser spectroscopy could help dissociating the different effect and conclude on the origin of the photoluminescence of silicon quantum dots.

\subsubsection{Influence of the nanocrystal size}

The nanoparticle size distribution is reduced with increasing strength of reducing agent. ${ }^{42,43}$ Upon injection of $\mathrm{LiAlH}_{4}$, a "burst nucleation" is expected. The strong reducing agent results in a fast reduction of the silicon precursor into monomers, thus limiting the 
nanocrystal growth. In comparison, the lower reactivity of $\mathrm{NaBH}_{4}$ induces a slower production of silicon monomers, and as the reduction time increases, the monomers are more likely to be contributing to particle growth. The particle size distribution is then larger when using a weaker reducing agent. $\mathrm{LiBH}_{4}$ has reactivity between $\mathrm{LiAlH}_{4}$ and $\mathrm{NaBH}_{4}$ so consequently the size distribution of the particles produced ranks from larger to smaller using $\mathrm{NaBH}_{4}, \mathrm{LiBH}_{4}$ then $\mathrm{LiAlH}_{4}$.

The shape of the nanocrystals is not influenced by the reactivity of the hydride reducing agent or the molecule attached to the surface of the quantum dots. The same spherical shape was observed for all the samples studied. In most of the synthesis reported in the literature, the silicon nanocrystals present a spherical shape. Plasma-mediated synthesis was reported to produce tetrahedral shaped silicon nanocrystals by the Kauzlarich group and Kortshagen et al reported cubic shaped also via a plasma process. ${ }^{44-46}$ In both cases, the particles were much larger than the ones reported in this study. The shape of nanocrystals is not controllable for particles with all dimensions smaller than $10 \mathrm{~nm} .{ }^{47}$

The absorption spectrum of the silicon nanocrystals obtained by reduction using $\mathrm{NaBH}_{4}$ present a unique peak at $210 \mathrm{~nm}$. The allylamine terminated silicon quantum dots synthesized via the chemical reduction using $\mathrm{LiBH}_{4}$ or $\mathrm{LiAlH}_{4}$ present two absorption peaks, the primary one being located at $210 \mathrm{~nm}$ for all the samples. The secondary peak varies from 265 to $300 \mathrm{~nm}$. It can be concluded that the first absorption peak correspond to an intrinsic property of the silicon nanocrystals, apparently independent of the nanoparticle size and associated to the bulk form of silicon. The secondary peak is observed only for the samples with a much smaller size, therefore it can be attributed to a pseudo direct band gap transition, occurring in particles with sizes in the quantum confinement regime.

All samples show a blue luminescence. However, the sample synthesized using $\mathrm{NaBH}_{4}$ exhibit a poor fluorescence compared to the other samples. As the size of those nanoparticles is much larger than the ones synthesized in the other experiments of this chapter, we can conclude that it is an evidence of quantum effects occurring in small silicon nanocrystals. Silicon nanocrystals with a size contained below the Bohr radius of $4.9 \mathrm{~nm}$ corroborate the theory of the pseudo-direct band gap. 


\subsubsection{Influence of the surface capping}

The surface capping agent used has no effect on the size and shape of the nanocrystals as it occurs in post-growth process. No variation of absorption wavelength for the secondary peak is observed for the three quantum-confined samples with different capping molecules, which evidences no absorption dependence upon the surface attachment.

The photoluminescence of the quantum-confined nanocrystals also does not vary depending on the surface capping molecule. The chemical termination of the passivation molecule (either amine or alkyl) as well as the carbon chain length of that molecule does not influence the fluorescence. The emission process can then be explained by the quantum confinement and by an electron transfer between the surface silicon atoms with their immediate neighbours (carbon or occasional nitrogen) via a covalent bond. 


\subsection{References}

1_Wilcoxon, J. P.; Samara, G. A.; Provencio, P. N., Optical and electronic properties of Si nanoclusters synthesized in inverse micelles. Physical Review B 1999, 60 (4), 2704.

2_ Warner, J. H.; Hoshino, A.; Yamamoto, K.; Tilley, R. D., Water-Soluble Photoluminescent Silicon Quantum Dots. Angewandte Chemie International Edition 2005, 44 (29), 4550-4554.

3_Shiohara, A.; Prabakar, S.; Faramus, A.; Hsu, C.-Y.; Lai, P.-S.; Northcote, P. T.; Tilley, R.

D., Sized controlled synthesis, purification, and cell studies with silicon quantum dots. Nanoscale 2011, 3 (8), 3364-3370.

4_ Veinot, J. G. C., Synthesis, surface functionalization, and properties of freestanding silicon nanocrystals. Chemical Communications 2006, 0 (40), 4160-4168.

5_Hessel, C. M.; Henderson, E. J.; Veinot, J. G. C., Hydrogen Silsesquioxane: A Molecular Precursor for Nanocrystalline Si-SiO2 Composites and Freestanding Hydride-SurfaceTerminated Silicon Nanoparticles. Chemistry of Materials 2006, 18 (26), 6139-6146.

6_Pettigrew, K. A.; Liu, Q.; Power, P. P.; Kauzlarich, S. M., Solution Synthesis of Alkyland Alkyl/Alkoxy-Capped Silicon Nanoparticles via Oxidation of Mg2Si. Chemistry of Materials 2003, 15 (21), 4005-4011.

7_Atkins, T. M.; Thibert, A.; Larsen, D. S.; Dey, S.; Browning, N. D.; Kauzlarich, S. M., Femtosecond Ligand/Core Dynamics of Microwave-Assisted Synthesized Silicon Quantum Dots in Aqueous Solution. Journal of the American Chemical Society 2011, 133 (51), 20664-20667.

8_Holmes, J. D.; Ziegler, K. J.; Doty, R. C.; Pell, L. E.; Johnston, K. P.; Korgel, B. A., Highly Luminescent Silicon Nanocrystals with Discrete Optical Transitions. Journal of the American Chemical Society 2001, 123 (16), 3743-3748. 
9_ Ding, Z.; Quinn, B. M.; Haram, S. K.; Pell, L. E.; Korgel, B. A.; Bard, A. J., Electrochemistry and Electrogenerated Chemiluminescence from Silicon Nanocrystal Quantum Dots. Science 2002, 296 (5571), 1293-1297.

10_Buriak, J. M., Silicon-Carbon Bonds on Porous Silicon Surfaces. advanced materials 1999, $11(3), 265-267$.

11_Buriak, J. M., Organometallic Chemistry on Silicon and Germanium Surfaces. Chemical Reviews 2002, 102 (5), 1271-1308.

12_Rogozhina, E. V.; Eckhoff, D. A.; Gratton, E.; Braun, P. V., Carboxyl functionalization of ultrasmall luminescent silicon nanoparticles through thermal hydrosilylation. Journal of Materials Chemistry 2006, 16 (15), 1421-1430.

13_Holmes, J. D.; Ziegler, K. J.; Doty, R. C.; Pell, L. E.; Johnston, K. P.; Korgel, B. A., Highly Luminescent Silicon Nanocrystals with Discrete Optical Transitions. Journal of the American Chemical Society 2001, 123 (16), 3743-3748.

14_Shiohara, A.; Hanada, S.; Prabakar, S.; Fujioka, K.; Lim, T. H.; Yamamoto, K.; Northcote, P. T.; Tilley, R. D., Chemical Reactions on Surface Molecules Attached to Silicon Quantum Dots. Journal of the American Chemical Society 2010, 132 (1), 248-253.

15_Sato, S.; Swihart, M. T., Propionic-Acid-Terminated Silicon Nanoparticles: Synthesis and Optical Characterization. Chemistry of Materials 2006, 18 (17), 4083-4088.

16_Erogbogbo, F.; Yong, K.-T.; Roy, I.; Xu, G.; Prasad, P. N.; Swihart, M. T., Biocompatible Luminescent Silicon Quantum Dots for Imaging of Cancer Cells. ACS Nano 2008, 2 (5), 873-878.

17_Warner, J. H.; Hoshino, A.; Shiohara, A.; Yamamoto, K.; Tilley, R. D., The synthesis of silicon and germanium quantum dots for biomedical applications - art. no. 609607. Colloidal Quantum Dots for Biomedical Applications 2006, 6096, 9607-9607

18_Krueger, K. M.; Al-Somali, A. M.; Falkner, J. C.; Colvin, V. L., Characterization of nanocrystalline CdSe by size exclusion chromatography. Anal. Chem. 2005, 77 (11), 3511-3515. 
19_Yu, W. W.; Chang, E.; Falkner, J. C.; Zhang, J.; Al-Somali, A. M.; Sayes, C. M.; Johns, J.;

Drezek, R.; Colvin, V. L., Forming Biocompatible and Nonaggregated Nanocrystals in Water Using Amphiphilic Polymers. Journal of the American Chemical Society 2007, 129 (10), 2871-2879.

20_Khorsand Zak, A.; Abd. Majid, W. H.; Abrishami, M. E.; Yousefi, R., X-ray analysis of ZnO nanoparticles by Williamson-Hall and size-strain plot methods. Solid State Sciences 2011, 13 (1), 251-256.

21_Shirahata, N., Colloidal Si nanocrystals: a controlled organic-inorganic interface and its implications of color-tuning and chemical design toward sophisticated architectures. Physical Chemistry Chemical Physics 2011, 13 (16), 7284-7294.

22_ Mastronardi, M. L.; Henderson, E. J.; Puzzo, D. P.; Ozin, G. A., Small Silicon, Big Opportunities: The Development and Future of Colloidally-Stable Monodisperse Silicon Nanocrystals. advanced materials 2012, 24 (43), 5890-5898.

23_Prtljaga, N.; D’Amato, E.; Pitanti, A.; Guider, R.; Froner, E.; Larcheri, S.; Scarpa, M.; Pavesi, L., Photoluminescence of hydrophilic silicon nanocrystals in aqueous solutions. Nanotechnology 2011, 22 (21), 215704.

24_Veinot, J. G. C., Synthesis, surface functionalization, and properties of freestanding silicon nanocrystals. Chemical Communications 2006, 0 (40), 4160-4168.

25_Dasog, M.; Yang, Z.; Regli, S.; Atkins, T. M.; Faramus, A.; Singh, M. P.; Muthuswamy, E.; Kauzlarich, S. M.; Tilley, R. D.; Veinot, J. G. C., Chemical Insight into the Origin of Red and Blue Photoluminescence Arising from Freestanding Silicon Nanocrystals. ACS Nano 2013, $7(3), 2676-2685$.

26_Dohnalova, K.; Poddubny, A. N.; Prokofiev, A. A.; de Boer, W. D. A. M.; Umesh, C. P.; Paulusse, J. M. J.; Zuilhof, H.; Gregorkiewicz, T., Surface brightens up Si quantum dots: direct bandgap-like size-tunable emission. Light Sci Appl 2013, 2, e47.

27_Prokofiev, A. A.; Moskalenko, A. S.; Yassievich, I. N.; Boer, W. D. A. M.; Timmerman, D.; Zhang, H.; Buma, W. J.; Gregorkiewicz, T., Direct bandgap optical transitions in Si nanocrystals. JETP Letters 2010, 90 (12), 758-762. 
28_Beard, M. C.; Knutsen, K. P.; Yu, P.; Luther, J. M.; Song, Q.; Metzger, W. K.; Ellingson, R. J.; Nozik, A. J., Multiple Exciton Generation in Colloidal Silicon Nanocrystals. Nano Letters 2007, 7 (8), 2506-2512.

29_Wilcoxon, J. P.; Samara, G. A., Tailorable, visible light emission from silicon nanocrystals. Applied Physics Letters 1999, 74 (21), 3164-3166.

30_Marini, A.; Muñoz-Losa, A.; Biancardi, A.; Mennucci, B., What is Solvatochromism? The Journal of Physical Chemistry B 2010, 114 (51), 17128-17135.

31_Leatherdale, C. A.; Bawendi, M. G., Observation of solvatochromism in CdSe colloidal quantum dots. Physical Review B 2001, 63 (16), 165315.

32_Liu, X.; Calata, J. N.; Liang, H.; Shi, W.; Lin, X.; Lin, K.; Qin, G. G., Photoluminescence and Photoluminescence Excitation Mechanisms for Porous Silicon and Silicon Oxynitride. MRS Online Proceedings Library 1999, 588

33_Noma, T.; Seol, K. S.; Kato, H.; Fujimaki, M.; Ohki, Y., Origin of photoluminescence around 2.6--2.9 eV in silicon oxynitride. Applied Physics Letters 2001, 79 (13), 1995-1997. 34_Zhang, L.; Shi, T.; Tang, Z.; Liu, D.; Xi, S.; Li, X.; Lai, W., Carbon-assisted growth and high visible-light optical reflectivity of amorphous silicon oxynitride nanowires. Nanoscale Research Letters 2011, 6 (1), 1-6.

35_Nekrashevich, S. S.; Gritsenko, V. A., Electronic structure of silicon oxynitride: Abinitio and experimental study, comparison with silicon nitride. Journal of Applied Physics 2011, 110 (11), 114103-6.

36_Rossi, A. M.; Murphy, T. E.; Reipa, V., Ultraviolet photoluminescence from $6 \mathrm{H}$ silicon carbide nanoparticles. Applied Physics Letters 2008, 92 (25), 253112-3.

37_Kagan, C. R.; Murray, C. B.; Nirmal, M.; Bawendi, M. G., Electronic Energy Transfer in CdSe Quantum Dot Solids. Physical Review Letters 1996, 76 (9), 1517-1520.

38_Dovrat, M.; Goshen, Y.; Jedrzejewski, J.; Balberg, I.; Sa'ar, A., Radiative versus nonradiative decay processes in silicon nanocrystals probed by time-resolved photoluminescence spectroscopy. Physical Review B 2004, 69 (15), 155311. 
38_TimmermanD; ValentaJ; DohnalovaK; de Boer, W. D. A. M.; GregorkiewiczT, Step-like enhancement of luminescence quantum yield of silicon nanocrystals. Nat Nano 2011, 6 (11), 710-713.

39_Hines, M. A.; Scholes, G. D., Colloidal PbS Nanocrystals with Size-Tunable NearInfrared Emission: Observation of Post-Synthesis Self-Narrowing of the Particle Size Distribution. advanced materials 2003, 15 (21), 1844-1849.

40_ LaMer, V. K.; Dinegar, R. H., Theory, Production and Mechanism of Formation of Monodispersed Hydrosols. Journal of the American Chemical Society 1950, 72 (11), 4847-4854.

41_ Norris, D. J.; Bawendi, M. G., Measurement and assignment of the size-dependent optical spectrum in CdSe quantum dots. Physical Review B 1996, 53 (24), 16338.

42_Prabakar, S.; Shiohara, A.; Hanada, S.; Fujioka, K.; Yamamoto, K.; Tilley, R. D., Size Controlled Synthesis of Germanium Nanocrystals by Hydride Reducing Agents and Their Biological Applications. Chemistry of Materials 2009, 22 (2), 482-486.

43_Zou, J.; Sanelle, P.; Pettigrew, K. A.; Kauzlarich, S. M., Size and Spectroscopy of Silicon Nanoparticles Prepared via Reduction of SiCl4. Journal of Cluster Science 2006, 17 (4), 565-578.

44_Atkins, T. M.; Thibert, A.; Larsen, D. S.; Dey, S.; Browning, N. D.; Kauzlarich, S. M., Femtosecond Ligand/Core Dynamics of Microwave-Assisted Synthesized Silicon Quantum Dots in Aqueous Solution. Journal of the American Chemical Society 2011, 133 (51), 20664-20667.

45_Baldwin, R. K.; Pettigrew, K. A.; Garno, J. C.; Power, P. P.; Liu, G.-y.; Kauzlarich, S. M., Room Temperature Solution Synthesis of Alkyl-Capped Tetrahedral Shaped Silicon Nanocrystals. Journal of the American Chemical Society 2002, 124 (7), 1150-1151.

46_Bapat, A.; Gatti, M.; Ding, Y.-P.; Campbell, S. A.; Kortshagen, U., A plasma process for the synthesis of cubic-shaped silicon nanocrystals for nanoelectronic devices. Journal of Physics D: Applied Physics 2007, 40 (8), 2247. 
47_ Tao, A. R.; Habas, S.; Yang, P., Shape Control of Colloidal Metal Nanocrystals. Small 2008, 4 (3), 310-325. 


\section{CZTS nanocrystals: synthesis and characterization}

$\mathrm{Cu}_{2} \mathrm{ZnSnS}_{4}$ (or $\mathrm{CZTS}$ ) has attracted considerable attention as candidate materials for the next generation of solar cells. This is because of their physical properties of direct band gap energy of $\mathrm{Eg} \approx 1.5 \mathrm{eV}$ and a large optical exctinction coefficient. ${ }^{1,2}$ Also CZTS are composed of copper, zinc, tin and sulphur, which are earth abundant elements. This makes CZTS a more economic and environmentally friendly option compared to the more widely studied structural analogue $\mathrm{CIS}\left(\mathrm{CulnS}(\mathrm{Se})_{2}\right)$ or $\left.\mathrm{CIGS}\left(\mathrm{Cu}_{2} \operatorname{lnGaS}\right)_{4}\right)^{3,4}$ Additionally, diminishing resources of indium encourage research towards an efficient earth abundant replacement, such as CZTS. Thin film solar cells fabricated from CZTS obtained by sputtering or vapour deposition techniques were found to have high conversion efficiencies up to $6.8 \% .^{5,6}$ However sputtering and vapour deposition techniques suffer from a substantial cost and therefore solution phase synthesis is a good way to significantly reduce the fabrication costs. ${ }^{7}$ So far, the reported synthesis methods for colloidal CZTS in solution involve the high temperature decomposition of metal salts sometimes involving hot injection. ${ }^{8,9}$

There is still a need for a size and shape control for CZTS nanocrystals. The only small CZTS nanoparticles exhibiting quantum confinement effect were reported by Khare in 2011. ${ }^{10}$ The solvent environment has been proven to have a strong influence over the size and shape of nanoparticles. ${ }^{11-13}$ In this research was first investigated the role of the solvent in high temperature synthesis. Next chemical reduction methods were attempted to prepare small monodisperse pure CZTS crystals.

Firstly, the effect of reaction temperature on the structure of CZTS nanocrystals was studied. The size and shape variation induced by a change in solvent media was investigated, from coordinating to non-coordinating. ${ }^{14}$ Then the effect of a reduction with a hydride reducing agent prior to heating treatment was examined.

Secondly, a chemical reduction method to synthesize CZTS nanoparticles was investigated. This method was adapted from that described in the previous chapter on 
silicon nanoparticles and the published work by the Tilley group regarding silicon and germanium quantum dots synthesis. It involves the reduction of metal salts using a strong hydride reducing agent at room temperature. The influence of a variation of molar ratio between the copper, zinc and tin precursors and the variation in crystals shape and composition between two different reducing agents were examined. Finally it was attempted to synthesize each metal sulphide individually using the same reduction synthesis process.

The nanoparticles were characterized by XRD, EDS, TEM and electron diffraction. The size distributions were calculated from the low resolution TEM images. The optical properties of the nanocrystals in solution were assessed using UV-Visible absorption spectroscopy.

\begin{tabular}{|c|c|c|c|}
\hline $\begin{array}{l}\text { Exp } \\
\text { No }\end{array}$ & $\begin{array}{l}\text { Synthetic } \\
\text { method }\end{array}$ & Effect researched & Specificity of each reaction \\
\hline 1 & \multirow{6}{*}{$\begin{array}{l}\text { Synthesis by } \\
\text { high } \\
\text { temperature } \\
\text { decomposition }\end{array}$} & Effect of temperature & $\begin{array}{l}\text { Aliquots taken at room temperature, } 180^{\circ} \mathrm{C} \text {, } \\
210^{\circ} \mathrm{C}, 260^{\circ} \mathrm{C} \text { and } 280^{\circ} \mathrm{C}\end{array}$ \\
\hline 2 & & \multirow{3}{*}{$\begin{array}{l}\text { Comparison of } \\
\text { different solvents }\end{array}$} & Oleylamine as solvent \\
\hline 3 & & & $50 / 50$ mixture of oleylamine and octadecene \\
\hline 4 & & & Octadecene as solvent \\
\hline 5 & & \multirow{2}{*}{$\begin{array}{l}\text { Effect of precursor } \\
\text { injection in hot solvent }\end{array}$} & Simultaneous injection of 4 precursors \\
\hline 6 & & & $\begin{array}{l}\text { Injection of zinc tin and sulphur precursors in } \\
\text { copper containing solution }\end{array}$ \\
\hline 7 & \multirow{11}{*}{$\begin{array}{l}\text { Synthesis by } \\
\text { chemical } \\
\text { reduction }\end{array}$} & \multirow{5}{*}{$\begin{array}{l}\text { Reduction by hydride } \\
\text { reducing agent prior to } \\
\text { heat treatment }\end{array}$} & No reducing agent (standard reaction) \\
\hline 8 & & & Using $\mathrm{LiBH}_{4}$ as a reducing agent \\
\hline 9 & & & Using $\mathrm{LiAlH}_{4}$ as a reducing agent \\
\hline 10 & & & $\begin{array}{l}\text { Using } \mathrm{LiBH}_{4} \text { in a larger solvent volume than exp } \\
8\end{array}$ \\
\hline 11 & & & $\begin{array}{l}\text { Using } \mathrm{LiAlH}_{4} \text { in a larger solvent volume than exp } \\
9\end{array}$ \\
\hline 12 & & \multirow{3}{*}{$\begin{array}{l}\text { Synthesis via reverse } \\
\text { micelles process }\end{array}$} & Reduction with $\mathrm{LiAlH}_{4}$ \\
\hline 13 & & & Reduction with $\mathrm{LiBH}_{4}$ \\
\hline 14 & & & $\begin{array}{l}\text { Reduction with } \mathrm{LiBH}_{4} \text { with a different precursors } \\
\text { relative ratio than } \exp 13\end{array}$ \\
\hline 15 & & \multirow{3}{*}{$\begin{array}{c}\text { Synthesis of single } \\
\text { metal sulphides using } \\
\mathrm{LiBH}_{4}\end{array}$} & $\mathrm{Cu}_{2} \mathrm{~S}$ \\
\hline 16 & & & ZnS \\
\hline 17 & & & SnS \\
\hline
\end{tabular}

Table 4.1: Description/comparison of the different experiments in this chapter 


\subsection{High temperature synthesis and influence of solvent environment}

In this section we are interested in the role the solvent environment has in the solution phase synthesis of CZTS. Other nanomaterials synthesis systems have been proven to be greatly influenced by either the solvent temperature or the ability of solvent/surfactant to bond to monomers or crystal faces, thus controlling the size and shape of the nanoparticles. ${ }^{15,16}$ First was investigated the morphology of nanocrystals taken from aliquots at five different temperatures lower than the usually reported temperature $\left(280^{\circ} \mathrm{C}\right)$ for CZTS synthesis. Then the particle size and structure of CZTS nanoparticles synthesized in solvents with different coordinating abilities were compared. Finally the direct injection of precursors into the hot solvent was studied.

\subsubsection{Effect of solvent temperature}

Reported solution-phase synthesis of CZTS nanocrystals are mostly conducted at $280^{\circ} \mathrm{C}$ or higher. In this section was studied the evolution of the shape and structure of CZTS nanocrystals at temperatures lower than $280^{\circ} \mathrm{C}$ to investigate what temperature is optimal for the formation of the CZTS structure. In this experiment, aliquots were taken at different temperatures, above room temperature. XRD, TEM and UV-Vis spectroscopy are used to estimate the structural and compositional nature of the nanocrystals. The synthetic protocol is based on the reported synthesis of CZTS nanocrystals by the Korgel group in $2009 .^{8}$

\section{Experimental}

\begin{tabular}{|c|c|c|c|c|c|c|}
\hline $\begin{array}{c}\text { Exp } \\
\text { No }\end{array}$ & $\mathrm{Cu}$ & $\mathrm{Zn}$ & Sn & $\mathbf{S}$ & Temperature & Solvent \\
\hline 1 & $\begin{array}{c}2 \\
\mathrm{mmol}\end{array}$ & $\begin{array}{c}1 \\
\mathrm{mmol}\end{array}$ & $\begin{array}{c}0.8 \\
\mathrm{mmol}\end{array}$ & $\begin{array}{c}4 \\
\mathrm{mmol}\end{array}$ & $\begin{array}{c}\text { aliquots taken at RT, 180, } \\
210,260 \text { and } 280\end{array}$ & Oleylamine \\
\hline
\end{tabular}

Table 4.2: Key variables in experiment 1

First, $0.52 \mathrm{~g}$ of copper acetylacetonate, $0.29 \mathrm{~g}$ of zinc acetate and $0.12 \mathrm{~g}$ of sulphur was weighed and dissolved in $40 \mathrm{~mL}$ of oleylamine in a three necked flask linked to a reflux condenser tube. The reaction vessel was degassed for 10 minutes under vacuum then purged with nitrogen for 30 minutes at room temperature. $0.8 \mathrm{~mL}$ of $\mathrm{SnCl}_{4}$ solution at 
$1 \mathrm{M}$ was injected in the solution. A first aliquot of $2 \mathrm{~mL}$ was withdrawn from the solution. The mixture was furthered degassed for another 30 minutes at $110^{\circ} \mathrm{C}$. The temperature was raised to $180^{\circ} \mathrm{C}$ and left for 10 minutes. A second $2 \mathrm{~mL}$ aliquot was taken. After 10 minutes at $210^{\circ} \mathrm{C}$, a third aliquot was abstracted. The fourth aliquot was taken after 10 minutes at $260^{\circ} \mathrm{C}$. The reaction was stopped after 15 minutes reaction at $280^{\circ} \mathrm{C}$ and let to cool down to room temperature naturally. Samples were purified by washing three times with a mixture methanol/toluene.

\section{Results}

XRD measurements allow the determination of the crystal structure of the synthesized nanoparticles. The XRD patterns in figure 4.1 show the evolution of the crystal structure of the nanocrystals over a range of temperatures from room temperature to $280^{\circ} \mathrm{C}$. The peak positions are compared to the ones reported in the JCPDS card 26-0575 for CZTS in the kesterite structure.

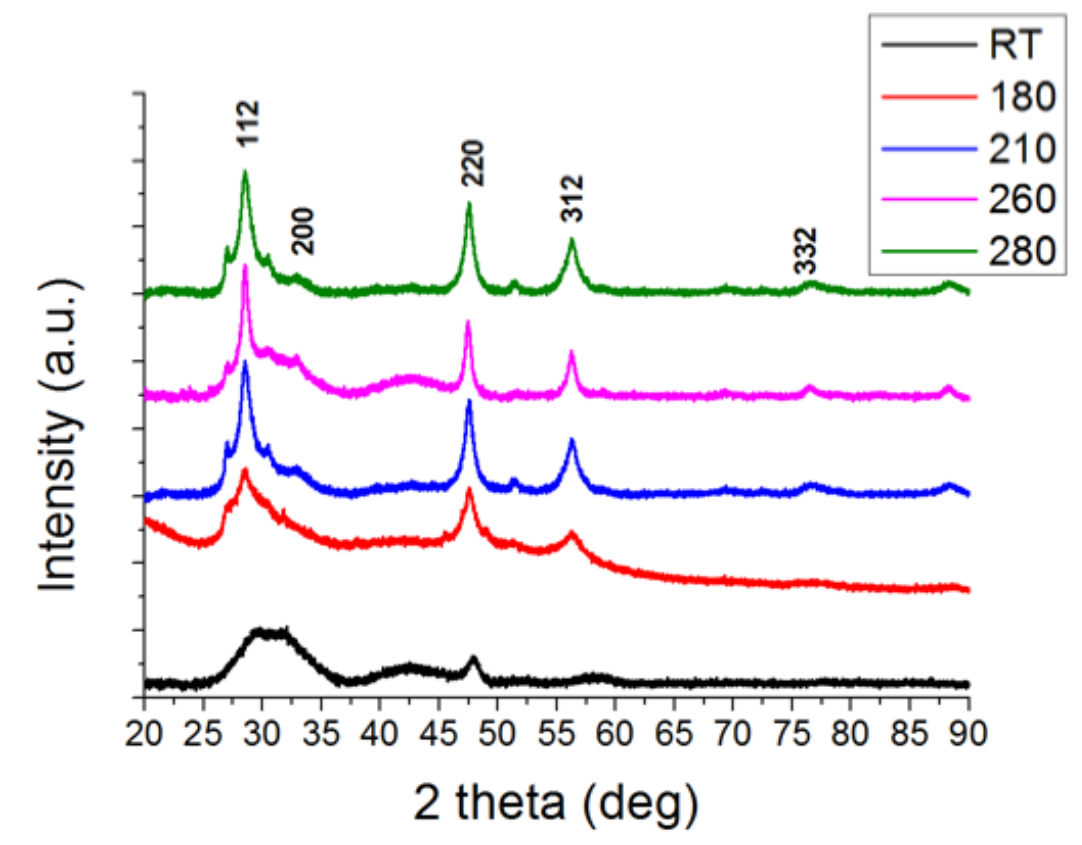

Figure 4.1: XRD spectra for the experiment 4 aliquots taken at room temperature, $180^{\circ} \mathrm{C}$, $210^{\circ} \mathrm{C}, 260^{\circ} \mathrm{C}$ and $280^{\circ} \mathrm{C}$.

The intensity of the peaks is increasing with increasing temperature, manifested as an increase in the crystallinity of the crystals. Starting from the small 2 theta angles on the 
left, the three major peaks observed on the spectra for the $180^{\circ} \mathrm{C}, 210^{\circ} \mathrm{C}, 260^{\circ} \mathrm{C}$ and $280^{\circ} \mathrm{C}$ correspond to the (112), (220) and (312) orientations of the kesterite crystal structure of CZTS. An amorphous impurity phase is observed on the spectrum for the aliquots taken at $260^{\circ} \mathrm{C}$ or lower. This amorphous phase is not observed for the particles obtained at the end of the reaction after treatment at $280^{\circ} \mathrm{C}$. A second phase of much weaker intensity can be observed on the XRD pattern of the particles treated at higher temperatures than $210^{\circ} \mathrm{C} . \mathrm{Cu}_{2} \mathrm{ZnSnS}_{4}$ can adopt the $\mathrm{ZnS}$ wurtzite structure, as demonstrated by the Yadong Li group in $2011 .{ }^{17}$ No standard data is available in the JCPDS database for CZTS in wurtzite arrangement, so we compare the peaks to the ZnS structure (JCPDS 75-1534), which have the same wurtzite configuration. The low intensity phase on our patterns can be matched to a wurtzite crystal structure. We can then conclude that our particles present a major kesterite type phase with a second phase that can be either wurtzite CZTS or an impurity of ZnS.

The nanocrystals obtained in the five aliquots were characterized by TEM imaging. The size and shape of the nanoparticles obtained in the different aliquots. The images and their corresponding size distribution estimated by measuring over 100 particles from different areas of the TEM grid are represented in figure 4.2. 


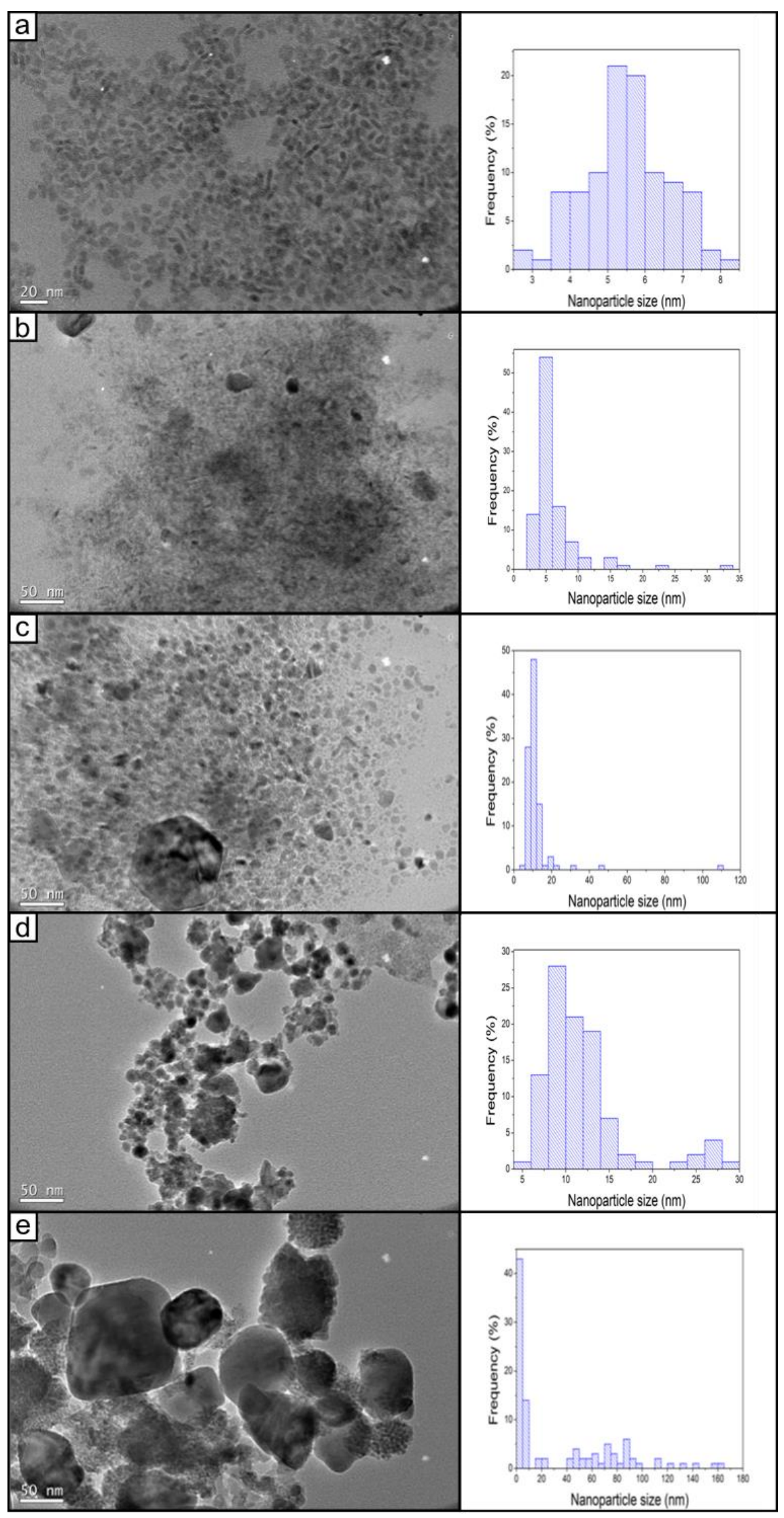

Figure 4.2: TEM images and size distribution for the aliquots taken at, from top to bottom: room temperature, $180^{\circ} \mathrm{C}, 210^{\circ} \mathrm{C}, 260^{\circ} \mathrm{C}$ and $280^{\circ} \mathrm{C}$. 
In figure 4.2.a, the particles taken in the aliquot taken at room temperature are monodisperse with a spherical shape. The average size is estimated to be $5.5 \pm 2.7 \mathrm{~nm}$. The particles from aliquot taken at $180^{\circ} \mathrm{C}$ can be seen in figure $4.2 . b$. The mean size of the nanocrystals is determined to be $6.3 \pm 25.7 \mathrm{~nm}$. The large majority of the particles present a small and spherical shape. A few particles are larger, over $30 \mathrm{~nm}$. In figure 4.2.c is represented the TEM image and the according size distribution of the particles collected in the aliquot taken at $210^{\circ} \mathrm{C}$. The particles are mostly spherical in shape. A few particles are faceted and of a large size, over $100 \mathrm{~nm}$. The average particle size is estimated to be $11.9 \pm 97.2 \mathrm{~nm}$. The $260^{\circ} \mathrm{C}$ aliquot (fig. 4.2.d) TEM image presents defined particles averaging in size $12.0 \pm 11.9 \mathrm{~nm}$. The crystals obtained after treatment at $260^{\circ} \mathrm{C}$ resemble the most the CZTS nanoparticles found in the literature. Finally, the particles obtained after heating treatment at $280^{\circ} \mathrm{C}$ present two types of particles: many small particles below $10 \mathrm{~nm}$ in size and big crystals, bringing the average size to $35.1 \pm$ $79.6 \mathrm{~nm}$ (fig. 4.2.e). The large disparity of size compared to similar methods with treatment at $280^{\circ} \mathrm{C}$ can be explained by the longer time to reach the final temperature, elongating the decomposition time of the precursors, and thus the nucleation time for the CZTS particles leading to a broadening of the particle size dispersion. ${ }^{18,19}$

The optical absorption of the nanocrystals in solution was measured by UV-Visible spectroscopy. The concentration was kept the same for all the samples. The absorption spectra from $350 \mathrm{~nm}$ to $1100 \mathrm{~nm}$ are determined, where the absorption edge is expected to appear. The gap energy was evaluated from the UV-visible absorption spectrum recorded using the Tauc plot (fig. 4.3.b). The direct band gap $E_{g}$, corresponds to the intersection point of the extrapolated straight portion of the curve $(A h v)^{2}$ vs. energy, with the abscissa axis, where $h v$ and $A$ measure the photon energy and the absorption coefficient, respectively. 


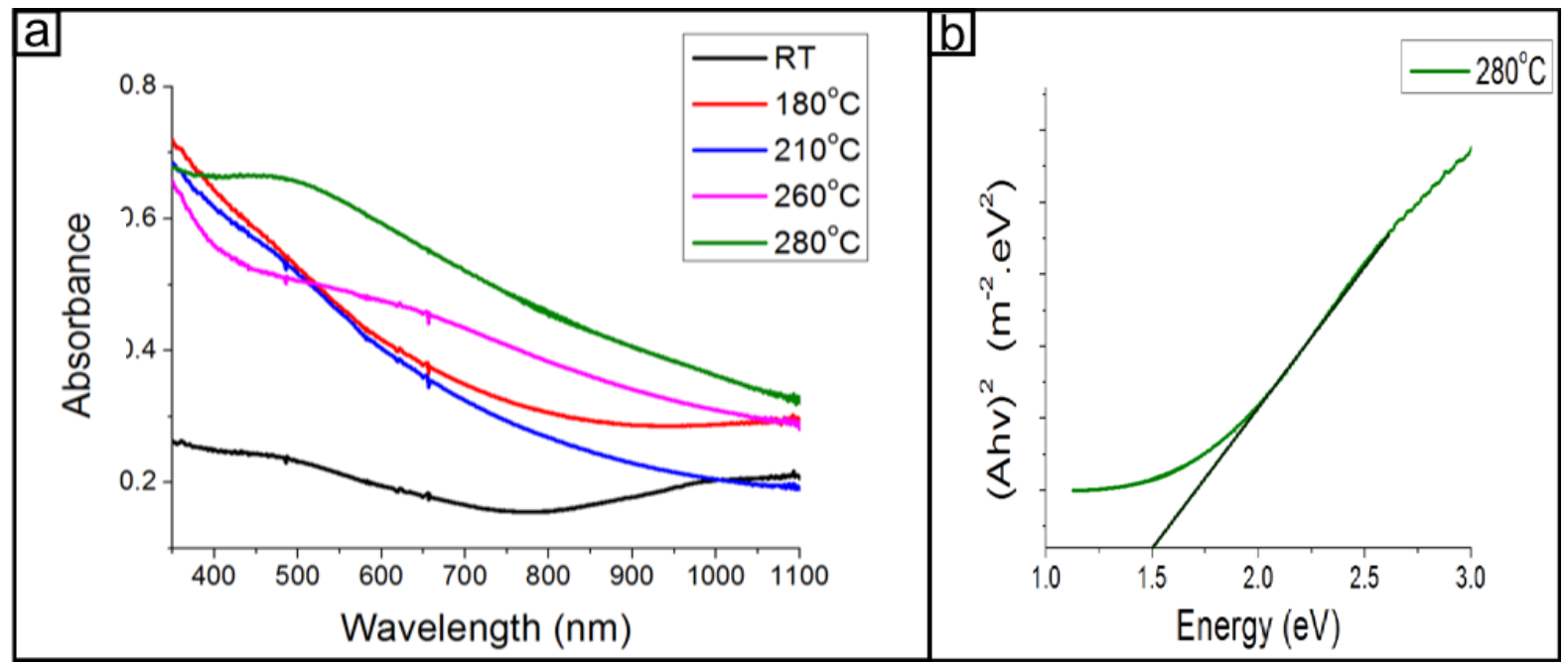

Figure 4.3: a) Optical absorption for the aliquots taken at room temperature, $180^{\circ} \mathrm{C}$, $210^{\circ} \mathrm{C}, 260^{\circ} \mathrm{C}$ and $280^{\circ} \mathrm{C}$ and $\left.\mathrm{b}\right)(A h v)^{2}$ as a function of photon energy to determine the bandgap of our nanocrystals obtained after the complete reaction $\left(280^{\circ} \mathrm{C}\right)$.

The shape of the absorption spectrum obtained for the nanocrystals after treatment at $180^{\circ} \mathrm{C}$ and $210^{\circ} \mathrm{C}$ are in good agreement with the reported absorption in the literature, with an absorption edge around $850 \mathrm{~nm}$, corresponding to a bandgap of $1.5 \mathrm{eV}^{20}$ The $(A h v)^{2}$ values, with $A$ being the absorption coefficient, $\mathrm{h}$ the Planck constant and $v$ the frequency, plotted as a function of photon energy can give an evaluation of the bandgap value by tracing an asymptotic line on the decreasing part of the curve (fig 4.3.b)). A value of $\mathrm{Eg}=1.5 \mathrm{eV}$ is determined. The measured bandgap is in good agreement with $\mathrm{Eg}$ values reported in the literature of $\mathrm{Eg} \approx 1.4-1.6 \mathrm{eV} \cdot{ }^{21,22} \mathrm{As} \mathrm{ZnS}$ has a bandgap of $3.6 \mathrm{eV}$ $(340 \mathrm{~nm})$, it can be concluded that the wurtzite structure observed with XRD experiments is in agreement with a wurtzite type structure of CZTS. ${ }^{23}$

In conclusion, the temperature of reaction influences the structure and size of the CZTS nanocrystals. The solvent temperature plays an important role in the formation of nanocrystals. A temperature of $260^{\circ} \mathrm{C}$ or higher is best to produce pure crystalline CZTS nanoparticles. In the next section will be evaluated the influence of the binding strength of solvents on the structure and shape of CZTS. 


\subsubsection{Effect of solvent coordination on CZTS nanocrystals formation}

In this section, the influence of the coordination ability of solvent on the size and shape of the CZTS nanoparticles is investigated. In other nanoparticle systems, the solvent or surfactant coordination has been shown to affect the size and shape of nanoparticles. ${ }^{13,24}$ During the synthesis, all the variables are kept constant, with the exception of the solvent. The solvents chosen were oleylamine and octadecene for their binding ability differences.

\section{Experimental}

The synthesis method is adapted from the work published by the Korgel group, using tin tetrachloride in solution as the tin precursor instead of tin dichloride in the reported synthesis.

\begin{tabular}{|c|c|c|c|c|c|c|c|}
\hline Exp No & $\mathbf{C u}$ & $\mathbf{Z n}$ & Sn & S & Temperature & Solvent & Surfactant \\
\hline 2 & $2 \mathrm{mmol}$ & $1 \mathrm{mmol}$ & $0.8 \mathrm{mmol}$ & $4 \mathrm{mmol}$ & 280 & Oleylamine & - \\
\hline 3 & $2 \mathrm{mmol}$ & $1 \mathrm{mmol}$ & $0.8 \mathrm{mmol}$ & $4 \mathrm{mmol}$ & 280 & $\begin{array}{c}\text { Oleylamine } \\
+ \\
\text { Octadecene }\end{array}$ & - \\
\hline 4 & $2 \mathrm{mmol}$ & $1 \mathrm{mmol}$ & $0.8 \mathrm{mmol}$ & $4 \mathrm{mmol}$ & 280 & Octadecene & - \\
\hline
\end{tabular}

Table 4.3: Key variables in experiments 2, 3 and 4

In a three necked flask, $0.52 \mathrm{~g}$ of copper acetylacetonate, $0.29 \mathrm{~g}$ of zinc acetate, $0.12 \mathrm{~g}$ of elemental sulphur were dissolved in either $40 \mathrm{~mL}$ of oleylamine, or $40 \mathrm{~mL}$ of octadecene or in a mixture of $20 \mathrm{~mL}$ of oleylamine and $20 \mathrm{~mL}$ of octadecene. After being degassed for 10 minutes under vacuum, the mixture was purged under nitrogen thirty minutes at room temperature, before $0.8 \mathrm{~mL}$ of tin tetrachloride was injected in the flask. The solution was brought to $120^{\circ} \mathrm{C}$ and left to degas for thirty minutes. The reaction mixture is heated at $280^{\circ} \mathrm{C}$ for one hour then cooled to room temperature. The nanoparticles were separated from solution using a centrifuge and were washed 3 times using a methanol/toluene mixture.

\section{Results}

The crystals produced in the three experiments described ahead were analysed by XRD to assess their crystalline structure. The peak positions are compared to the ones reported in the JCPDS card 26-0575 for CZTS in the kesterite structure. Using the 
Scherrer equation from the spectra in figure 4.4 , the average particle size of the nanocrystals of each of the three samples from experiments 2,3 and 4 is evaluated. ${ }^{24}$

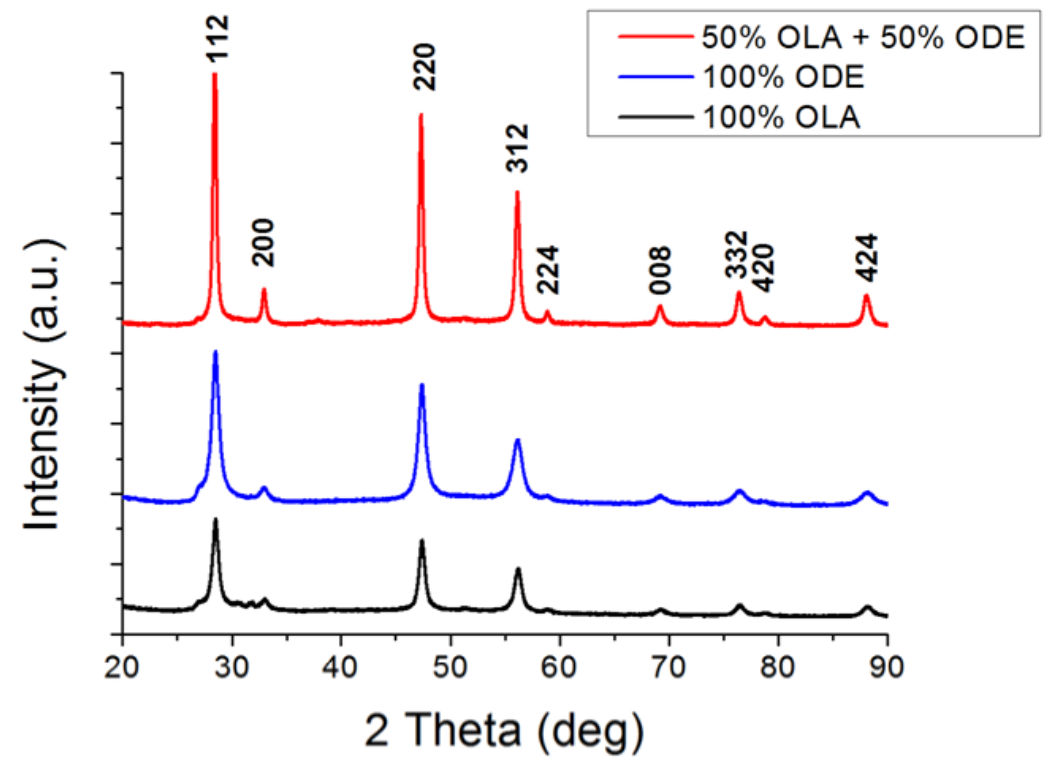

Figure 4.4: XRD spectrum of CZTS synthesized in a mixture of oleylamine and octadecene.

All three spectra show a pure kesterite type crystalline phase of CZTS. The peak positions and intensities match the JCPDS standard. No impurity phase of wurtzite structure of CZTS is observed. According to the particle size determination using the Scherrer equation, the particles synthesized in oleylamine had dimensions of $13.2 \mathrm{~nm}$, $22.8 \mathrm{~nm}$ when synthesized in octadecene and $30.4 \mathrm{~nm}$ when using a mixture OLA/ODE as a solvent. We would expect the particle size to be the largest when using only octadecene as it is the solvent with the lower binding ability. TEM imaging give a more accurate estimation of the nanoparticles sizes for those three samples.

Figure 4.5 shows a typical image for each of the sample prepared using oleylamine, octadecene or a mixture oleylamine/octadecene as a solvent and their corresponding size distribution. 


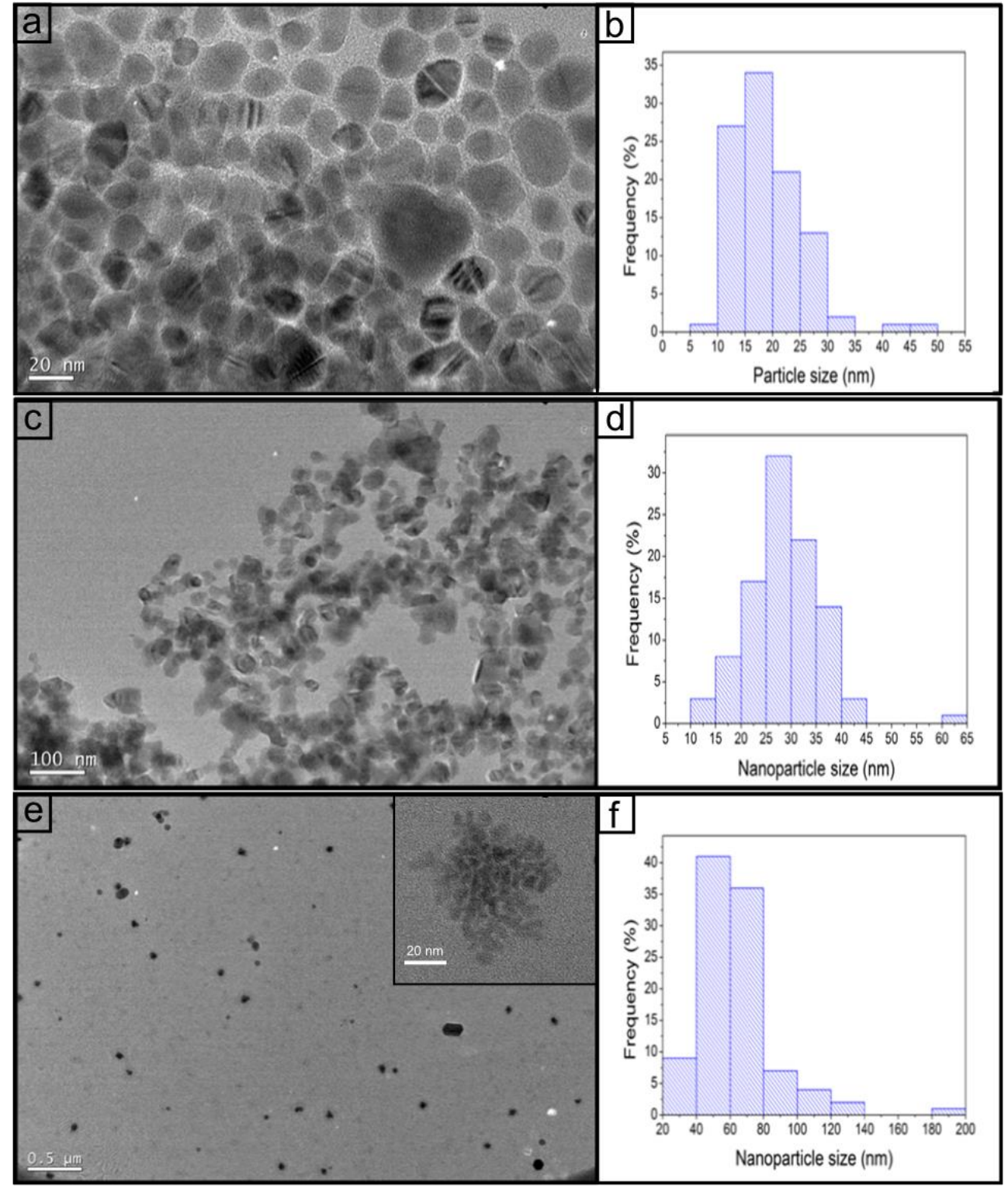

Figure 4.5: Low resolution TEM images of the CZTS nanocrystals synthesized in oleylamine (a), octadecene/oleylamine mixture (c) and octadecene (e) and (b), (d), (f) represents their corresponding particle size distribution.

Most particles present a spherical shape and some faceted shapes for particles synthesized in oleylamine. Using an OLA/ODE mixture, the shape appears to be less regular than in pure OLA. The particles remain mostly spherical. After synthesis in ODE, the particles present a variety of shapes, from large hexagons, to small spherical crystals and porous-like particles as we can see in the inset of figure 4.5.e). In figure 4.5.b), the 
average diameter of the nanocrystals is $19 \pm 24 \mathrm{~nm}$ when using oleylamine as a solvent. The average size of the nanocrystals synthesized in the mixture $1 / 1$ oleylamine/octadecene is determined to be $29 \pm 25 \mathrm{~nm}$. The average size of the nanocrystals synthesized in ODE is estimated to be $64 \pm 82 \mathrm{~nm}$. The inset in figure 4.5.e) shows that some particles, under higher magnification, present a structure with dendrites.

Both XRD and TEM analysis prove the increase of particle size when switching from oleylamine to octadecene as a solvent. With a larger coordination strength due to the amine termination and a longer carbon chain, oleylamine acts as a surfactant and thus limits the growth of the crystals by stabilizing the surface of the nanoparticles. Octadecene lead to a larger overall nanoparticle size, confirming the weak coordination ability. The presence of the multigrain structures observed for some particles grown in dodecene would lower the particle size determined by XRD as it measures the size of each crystallite domain.

In conclusion, the solvent temperature permits to control the size, shape and composition of the CZTS nanocrystals and the solvent coordination ability has a strong influence on the size and shape of the nanoparticles. These observations are in good agreement with the literature reports on $\mathrm{CZTS}^{17}$ and other systems. ${ }^{14,16}$

\subsubsection{Synthesis by hot injection of precursors}

Experiments 5 and 6 were conducted via the hot injection method. It is proposed to inject the precursors in solution into a preheated solvent to facilitate a burst nucleation. Injecting the precursors swiftly in the hot solvent was aimed at achieving a better size control of the nanoparticles. The solvent, temperature and molar ratios of precursors were kept constant. The two syntheses differ in the injection process. In experiment 5 , all precursors were injected simultaneously in the hot solvent and in experiment 6 , the copper precursor was already in solution before injection of the three other precursors. These experiments are based on the method reported by the Prieto group. ${ }^{9}$ 
Experimental

\begin{tabular}{|c|c|c|c|c|c|c|c|}
\hline $\begin{array}{c}\text { Exp } \\
\text { no }\end{array}$ & $\mathbf{C u}$ & $\mathbf{Z n}$ & Sn & S & Temperature & $\begin{array}{c}\text { Solvent / } \\
\text { Surfactant }\end{array}$ & Injection \\
\hline 5 & $\begin{array}{c}0.5 \\
\mathrm{mmol}\end{array}$ & $\begin{array}{c}0.3 \\
\mathrm{mmol}\end{array}$ & $\begin{array}{c}0.2 \\
\mathrm{mmol}\end{array}$ & $\begin{array}{c}1 \\
\mathrm{mmol}\end{array}$ & 280 & $\begin{array}{c}\text { Oleylamine } \\
\text { and Oleic acid }\end{array}$ & $\begin{array}{c}\text { All } 4 \\
\text { precursors }\end{array}$ \\
\hline 6 & $\begin{array}{c}0.5 \\
\mathrm{mmol}\end{array}$ & $\begin{array}{c}0.3 \\
\mathrm{mmol}\end{array}$ & $\begin{array}{c}0.2 \\
\mathrm{mmol}\end{array}$ & $\begin{array}{c}1 \\
\mathrm{mmol}\end{array}$ & 280 & $\begin{array}{c}\text { Oleylamine } \\
\text { and Oleic acid }\end{array}$ & $\begin{array}{c}\mathrm{S}+\mathrm{Zn} \text { and } \\
\mathrm{Sn} \\
\text { precursors }\end{array}$ \\
\hline
\end{tabular}

Table 4.4: Key variables in experiment 5 and 6

In both experiments, $10 \mathrm{~mL}$ of solution composed of $1 / 1$ ratio of oleylamine and oleic acid was prepared. In experiment 5, the four precursors were dissolved in the prepared solution in separated vials sealed with a septum degassed for 2 minutes and then purged with nitrogen for 10 minutes. $0.13 \mathrm{~g}$ of copper acetylacetonate was mixed in 2 $\mathrm{mL}$ of the solution, $0.075 \mathrm{~g}$ of zinc acetate in $1 \mathrm{~mL}, 0.2 \mathrm{~mL}$ of $1 \mathrm{M}$ solution of tin tetrachloride in tetrahydrofuran in $1 \mathrm{~mL}$ of mixture OLA/OA and $0.03 \mathrm{~g}$ of sulphur in 1 $\mathrm{mL}$. The 4 vials are heated to $150^{\circ} \mathrm{C}$. In a $50 \mathrm{~mL}$ three necked flask equiped with a reflux condenser, $5 \mathrm{~mL}$ of the mixture OLA/OA was brought to $310^{\circ} \mathrm{C}$. The four precursors were injected simultaneously in the flask. The mixture was then kept at $280^{\circ} \mathrm{C}$ for one hour. In experiment 6, the copper precursor was present in the reaction solution before heating, the zinc, tin and sulfur sources were hot injected. $0.075 \mathrm{~g}$ of zinc acetate, 0.2 $\mathrm{mL}$ of $1 \mathrm{M}$ solution of tin tetrachloride in THF and $0.03 \mathrm{~g}$ of sulphur in $3 \mathrm{~mL}$ of OLA/OA mixture were mixed in a sepparate vial with a septum degassed for 2 minutes and then purged with nitrogen for 10 minutes then heated to $150^{\circ} \mathrm{C}$. In a $50 \mathrm{~mL}$ three necked flask equiped with a condensation tube, $0.13 \mathrm{~g}$ of copper acetylacetonate was mixed to $7 \mathrm{~mL}$ of the mixture OLA/OA and degassed for 20 minutes then purged with nitrogen for 30 minutes. The mixture was brought to $320^{\circ} \mathrm{C}$. The three other precursors were injected simultaneously in the flask. The mixture was then kept at $280^{\circ} \mathrm{C}$ for one hour. In both experiments, after slowly cooling down to room temperature, the CZTS nanoparticles were separated from solution by centrifugation and washed three times with a mixture methanol/toluene. 


\section{Results for experiment 5: hot injection of the 4 precursors}

The crystal structure of the crystals was determined by X-ray diffraction. The purified nanocrystals were packed as a powder in a low background sample holder. The XRD spectrum is presented of experiment 5 in figure 4.6.

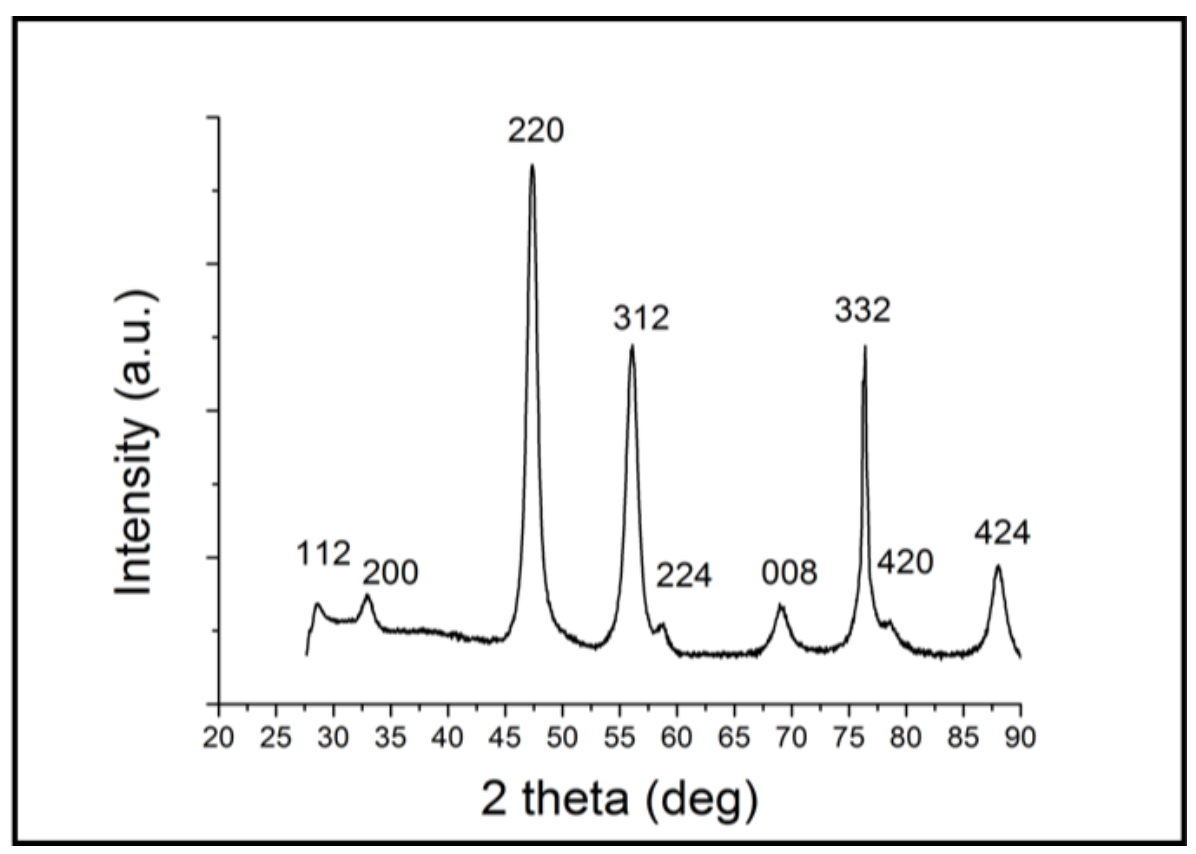

Figure 4.6: XRD pattern of CZTS obtained by simultaneous hot injection of the copper, zinc and tin precursor and sulphur.

The crystal structure of the nanoparticles obtained after simultaneous injection of the four precursors at high temperature matches the kesterite CZTS crystal structure. No crystalline impurity phase is observed and the sharp profile of the peaks attest of the pure nature of the CZTS crystals formed in experiment 5. The intensity of the (112) reflection is lower than expected due to the background elimination, higher for smaller 2 theta values when amorphous impurities are present.

The size and shape of the crystals was then evaluated by transmission electron microscopy. 


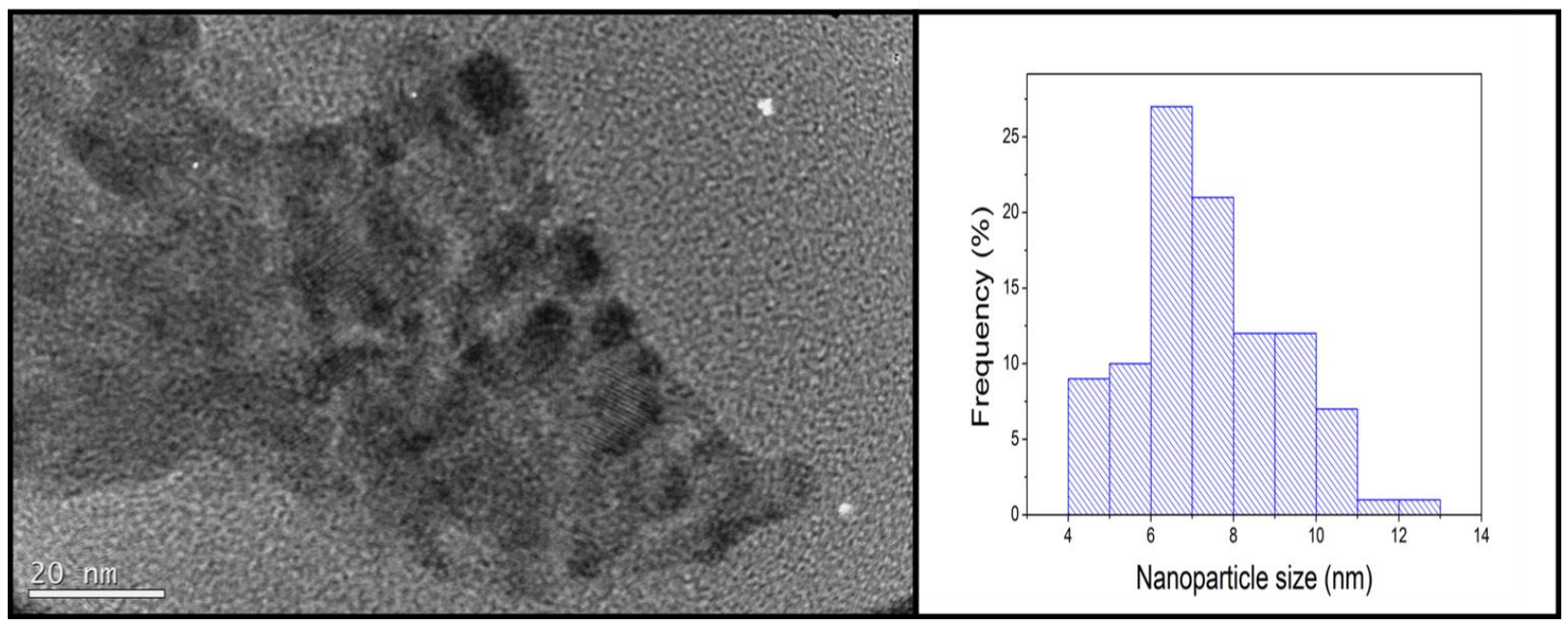

Figure 4.7: TEM image of the CZTS nanocrystals from experiment 5 and their corresponding size distribution.

The shape of the particles is mostly spherical. The average size, evaluated by measuring over 200 particles from different areas of the TEM grid, is $6.8 \pm 4.6 \mathrm{~nm}$. The average size of the nanoparticles is smaller than reported CZTS nanocrystals reported by similar methods in the literature. ${ }^{8,20}$ The synthesis by hot injection of metal precursors leads to the formation of CZTS nanocrystals, with a much smaller average diameter size than the CZTS synthesized by thermal decomposition of precursors in solution heated together to $280^{\circ} \mathrm{C}$. The hot injection permits the rapid decomposition of the copper, tin, zinc and sulphur precursors, leading to a nucleation closer to the "burst nucleation" phenomenon, which occurs when monomers are formed simultaneously, limiting the size distribution and the size variability during the growth process. ${ }^{25}$

The optical UV-Vis absorption of the CZTS nanocrystals obtained in experiment 5 is represented in figure 4.8. The nanoparticles were dispersed in a methanol solution. 


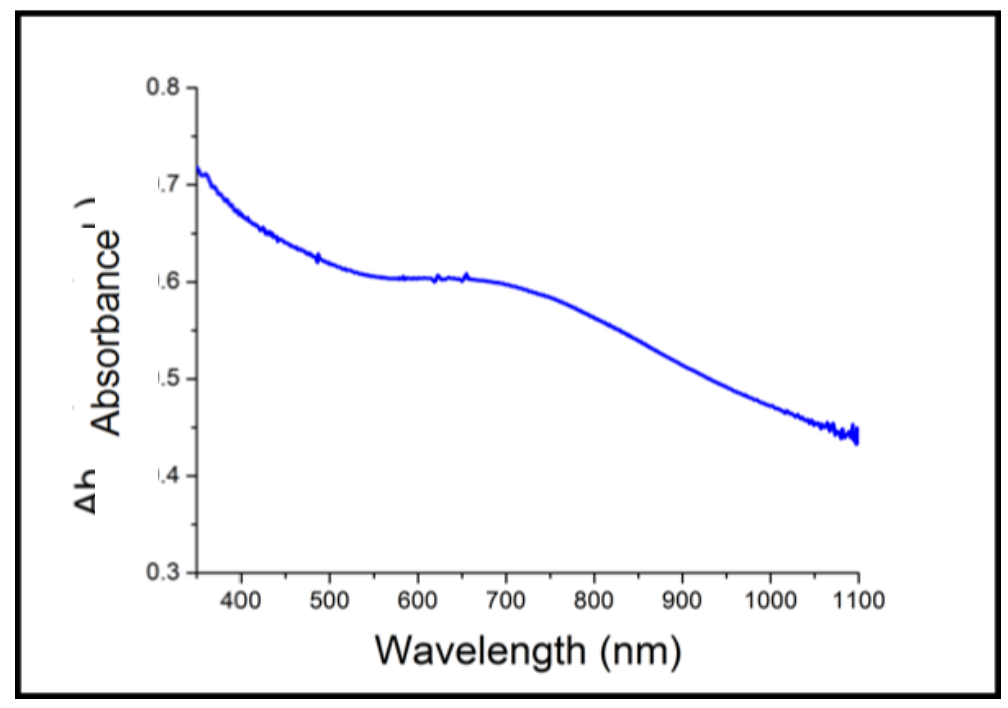

Figure 4.8: Optical absorption of CZTS nanocrystals synthesized by hot injection of the 4 precursors in solution.

The optical absorption spectrum has shape consistent with reports of CZTS nanocrystals in the literature. The shoulder at $750 \mathrm{~nm}$ and the absorption edge around $900 \mathrm{~nm}$ is in agreement with the bandgap for CZTS of 1.4-1.5 eV.

The simultaneous injection of 4 elements was successful in the synthesis of small pure kesterite structure CZTS. Nevertheless, the synthetic process is not trivial and requires extreme care. In addition, two persons were required to simultaneously inject the four precursors. To facilitate the synthesis, the next experiment was conducted by injection of three of the four precursors premixed in a separated vial.

Results for experiment 6: hot injection of zinc, tin and sulphur precursors in copper containing hot solution

Zinc, tin and sulphur precursors were injected in the copper containing solution. The copper was in the initial solution as it has the largest volume and copper easily complexes with oleylamine from room temperature.

Figure 4.9 represents the XRD pattern obtained for the crystals in experiment 6 . 


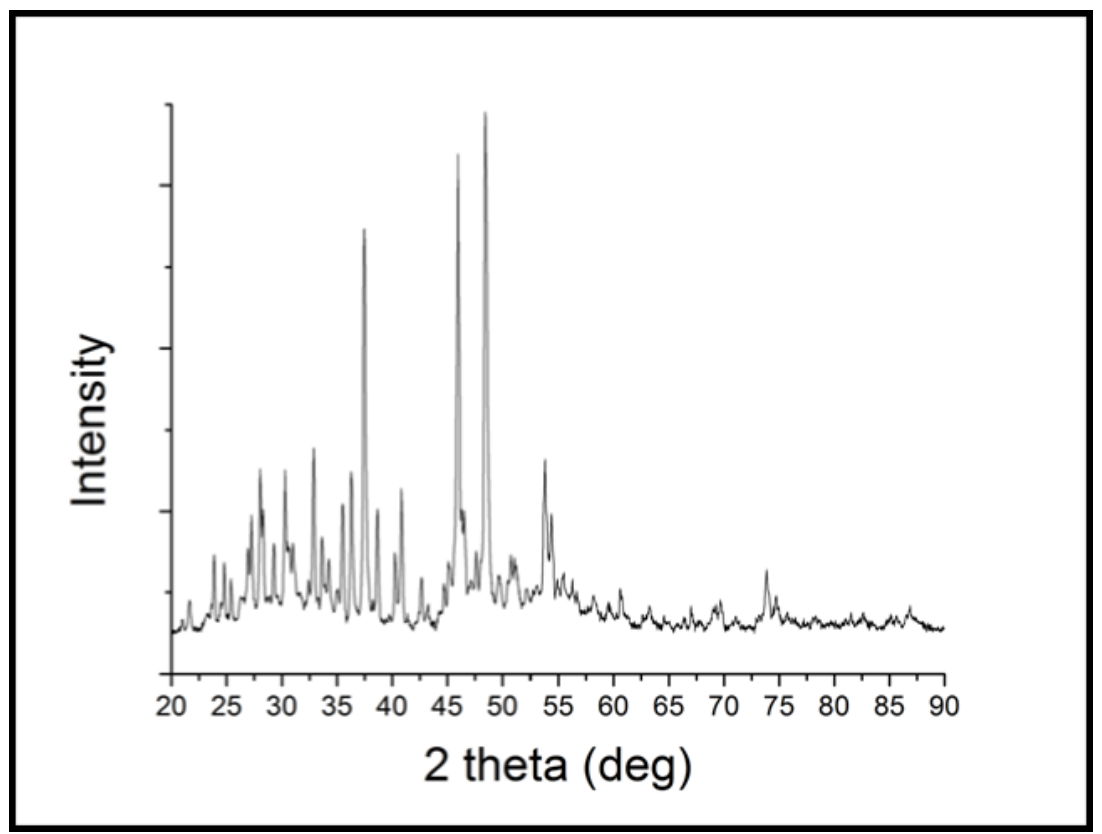

Figure 4.9: XRD pattern for the CZTS synthesis via the hot injection of the tin, zinc and sulphur precursor in the hot solvent containing the copper precursor.

The XRD spectrum of particles obtained in experiment 6 presents a multitude of peaks, corresponding to more than a single crystal phase. The kesterite CZTS structure is observed, but additional structures are also observed. The main additional phases can be matched to the $\mathrm{Cu}_{2} \mathrm{~S}$ and $\mathrm{SnS}$ structures. Some peaks can also be matched to $\mathrm{ZnS}, \mathrm{Sn}$ and elemental sulphur. The intensities of those other structures are comparable or higher that of the kesterite type crystalline phase.

Figure 4.10 pictures the TEM image of the nanocrystals for the experiment featuring the simultaneous injection of the three $\mathrm{Sn}, \mathrm{Zn}$ and $\mathrm{S}$ precursors in the copper containing hot solvent. 


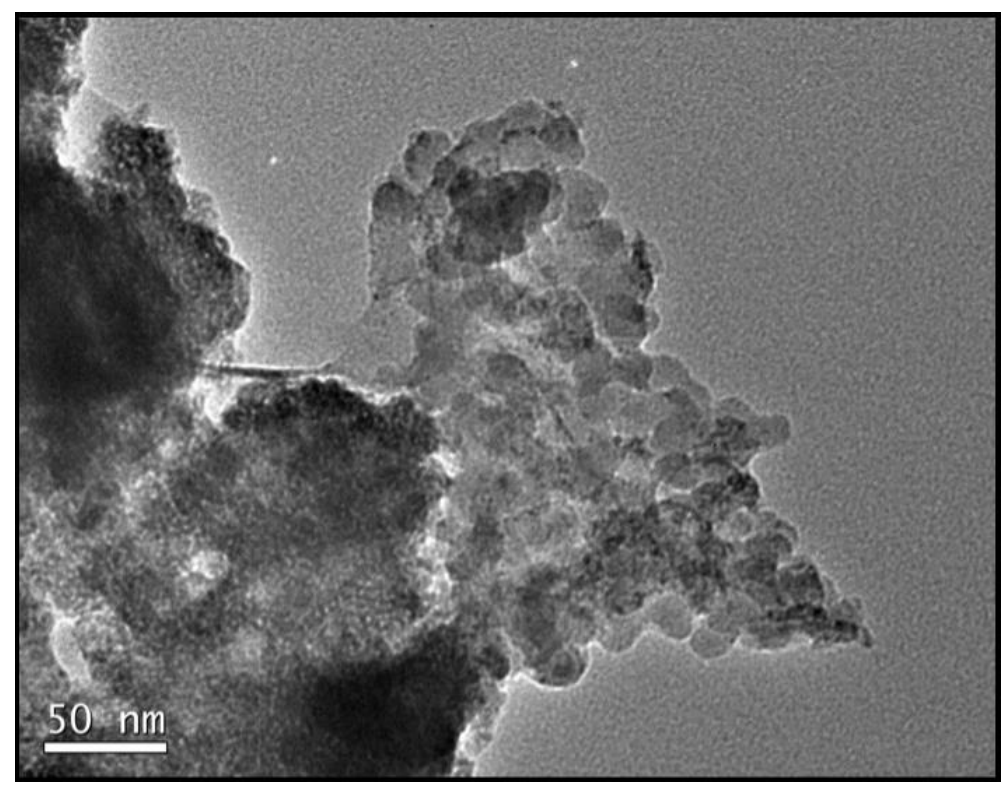

Figure 4.10: Low resolution TEM image of the nanocrystals obtained with experiment 7.

Even after purification and ultrasonic dispersion of the nanoparticle solution, the TEM image in figure 4.10 shows an aggregation of particles and organic matter. The nanoparticle size was not measurable.

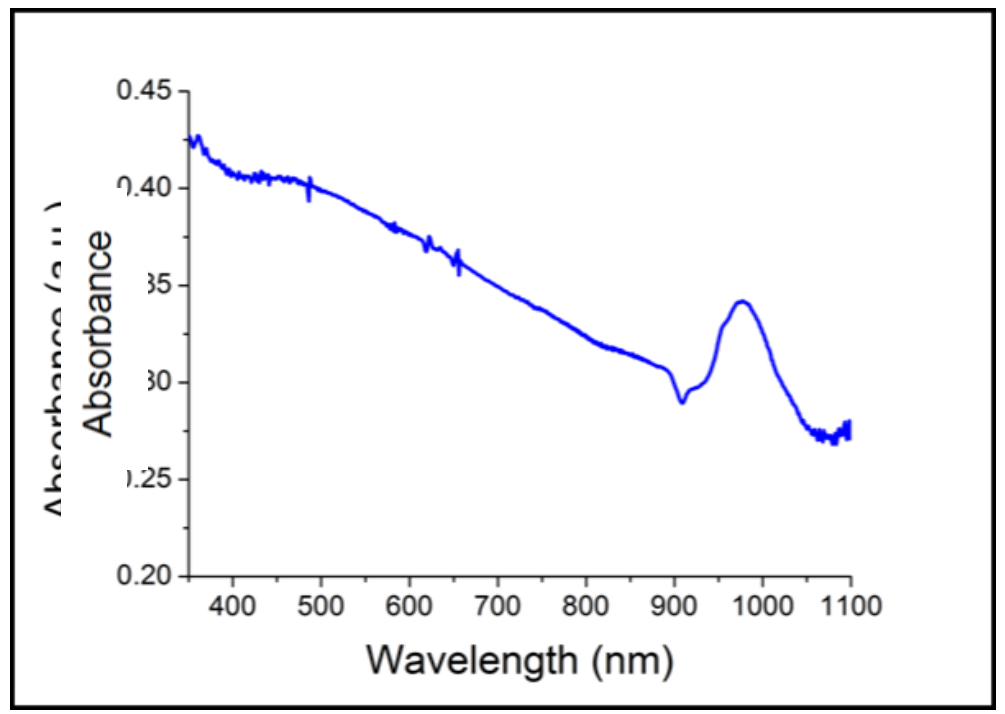

Figure 4.11: Optical UV-Visabsorption of CZTS nanocrystals synthesized by hot injection of the zinc, tin and sulphur precursors in solution containing the copper precursor. 
The optical UV-Vis absorption graph shows an absorption over the entire wavelength range. The peak at $990 \mathrm{~nm}$ with an absorption edge at $1100 \mathrm{~nm}$ corresponds to the transitions in SnS (bandgap $1.2 \mathrm{eV}$ ). ${ }^{26}$ The shape of the spectrum is consistent with the result from X-ray diffraction that several crystal structures are present in the sample.

The hot injection of the zinc, tin and sulphur precursors into the hot solvent containing the copper precursor lead to the formation of nanocrystals. The XRD analysis reveals several crystal structures. The CZTS kesterite structure is present, but a large amount of impurities phases of $\mathrm{Cu}_{2} \mathrm{~S}$ and metallic $\mathrm{Sn}$ have also being synthesized in this experiment among other impurities. The multitude of structures is a result of a widened precursor decomposition process. The copper acetylacetonate was already decomposed into copper monomers when the zinc acetate, tin tetrachloride and sulphur were injected. The sulphur atoms reacted first with the copper monomers, forming copper sulfide. As the decomposition of the tin tetrachloride happened with a slight delay, the tin monomers formed metallic tin nanoparticles.

The injection of tin, zinc and sulphur precursors into the hot solvent containing the copper precursor did not produce a single type of CZTS nanocrystals but a variety of crystal phases. The copper precursor was most probably decomposed before the injection of the other precursors, creating a multi-nucleation event that lead to several metallic or single metal sulphide phases.

To reduce the decomposition time discrepancy between the different precursors, the use of a reducing agent prior to the heating process could enhance the monomer production process and help to control the size of CZTS nanocrystals.

\subsection{Chemical reduction synthesis of CZTS}

\subsubsection{Experiments 7 to 11: Use of a reducing agent prior to high} temperature treatment

Experiments 7, 8 and 9 were conducted to compare the CZTS nanocrystals produced with or without a reducing agent before the high temperature treatment. Experiment 7 
is the reaction used as a reference. In Experiment 8, lithium borohydride was used to reduce the precursors before heating. Finally, in experiment 9, the precursors were reduced using lithium aluminium hydride. The use of the reducing agent is expected to help the decomposition process of the $\mathrm{Cu}, \mathrm{Sn}$ and $\mathrm{Zn}$ precursors. In experiment 6 , the decomposition time disparity led to large particle size. Using a reducing agent, we expect to reduce the average particle size. $\mathrm{LiAlH}_{4}$ is a stronger reducing agent than $\mathrm{LiBH}_{4}$, so it is expected that it will further reduce the particle size and variability of size (refer to chapter 3.3). ${ }^{27}$

\section{Experiment 7: No reducing agent}

In this experiment, the four precursors were mixed in oleylamine before the heating treatment. It will be used as a standard for comparison with experiments 8 and 9 .

\section{Experimental}

\begin{tabular}{|c|c|c|c|c|c|c|c|}
\hline $\begin{array}{c}\text { Exp } \\
\text { No }\end{array}$ & Cu & Zn & Sn & S & Temperature & Solvent & Surfactant \\
\hline 7 & $\begin{array}{c}0.5 \\
\mathrm{mmol}\end{array}$ & $\begin{array}{c}0.3 \\
\mathrm{mmol}\end{array}$ & $\begin{array}{c}0.2 \\
\mathrm{mmol}\end{array}$ & $\begin{array}{c}1 \\
\mathrm{mmol}\end{array}$ & 280 & Oleylamine & - \\
\hline
\end{tabular}

Table 4.5: Key variables in experiment 7

$0.099 \mathrm{~g}$ of copper acetate, $0.075 \mathrm{~g}$ of zinc acetate, $0.045 \mathrm{~g}$ of tin dichloride and $0.035 \mathrm{~g}$ of elemental sulfur were ground together in a mortar and suspended in $10 \mathrm{~mL}$ of oleylamine.The solution has a dark brown colour. The mixture is transferred into a 50 $\mathrm{mL}$ three necked flask. The mixture is degassed under vacuum for $1 \mathrm{~h}$ at room temperature then purged with nitrogen for 15 minutes followed by an hour of nitrogen flow at $120^{\circ} \mathrm{C}$. The temperature is then raised to $280^{\circ} \mathrm{C}$ and the mixture was left to react for one hour. The system was slowly cooled to room temperature. $5 \mathrm{~mL}$ of ethanol was added to the mixture to facilitate the separation of the nanoparticles. The solid was collected by centrifugation at $140.000 \mathrm{rpm}$ for 2 minutes. The nanoparticles were washed 3 times in a mixture 1/1 methanol/toluene.

\section{Results}

Figure 4.12 represents the $\mathrm{X}$-ray diffraction spectrum obtained from the nanoparticles in experiment 7 after purification and drying. The peak positions are compared to the ones reported in the JCPDS card 26-0575 for CZTS in the kesterite structure. 


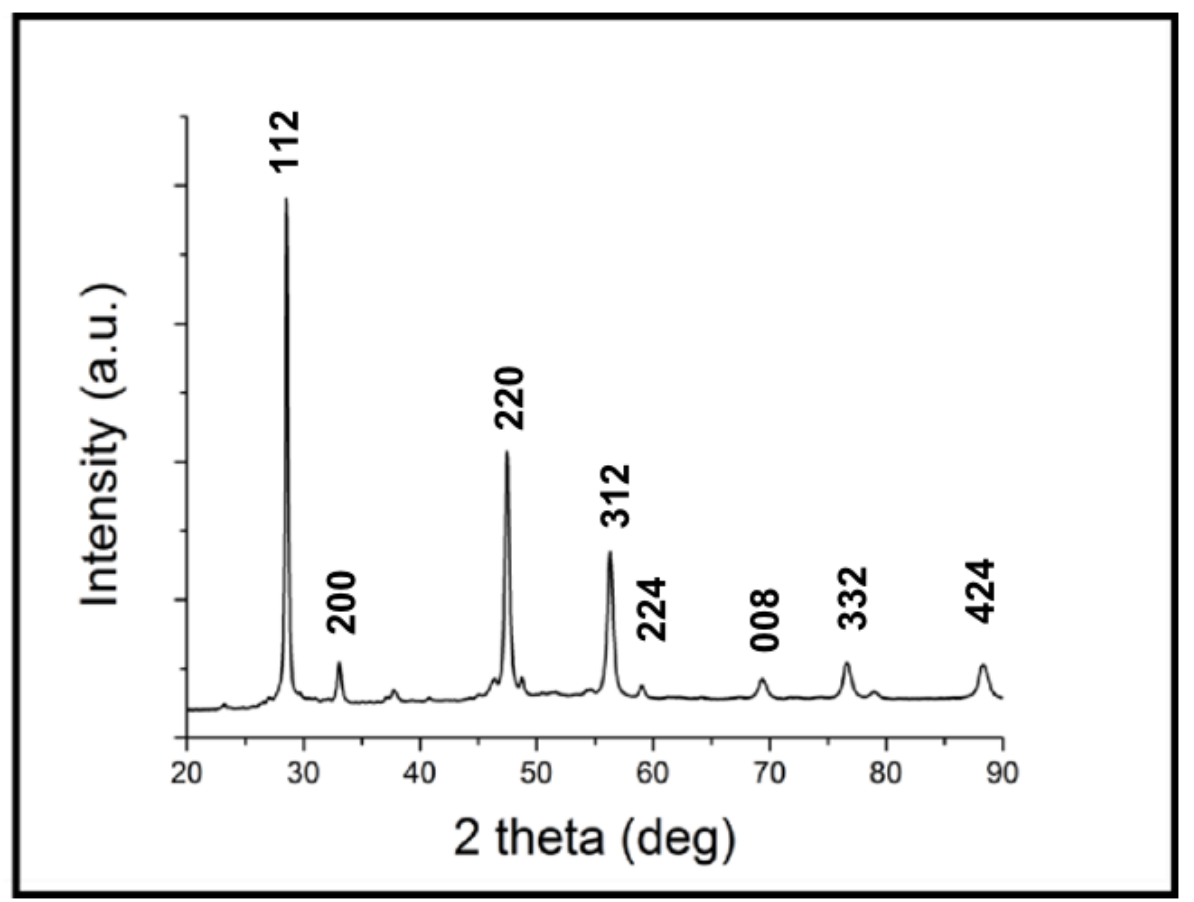

Figure 4.12: XRD pattern obtained for experiment 7.

The crystal structure of the nanocrystals matches the tetragonal kesterite structure. The peak positions and intensities matches the standard in the JCPDS card. It presents all the reflection expected in the 2 theta angles range of $20-90^{\circ}$.

The sizes and shapes were determined with TEM imaging. Electron diffraction spectroscopy associated to the TEM gives information about the crystal structure of the nanoparticles. 


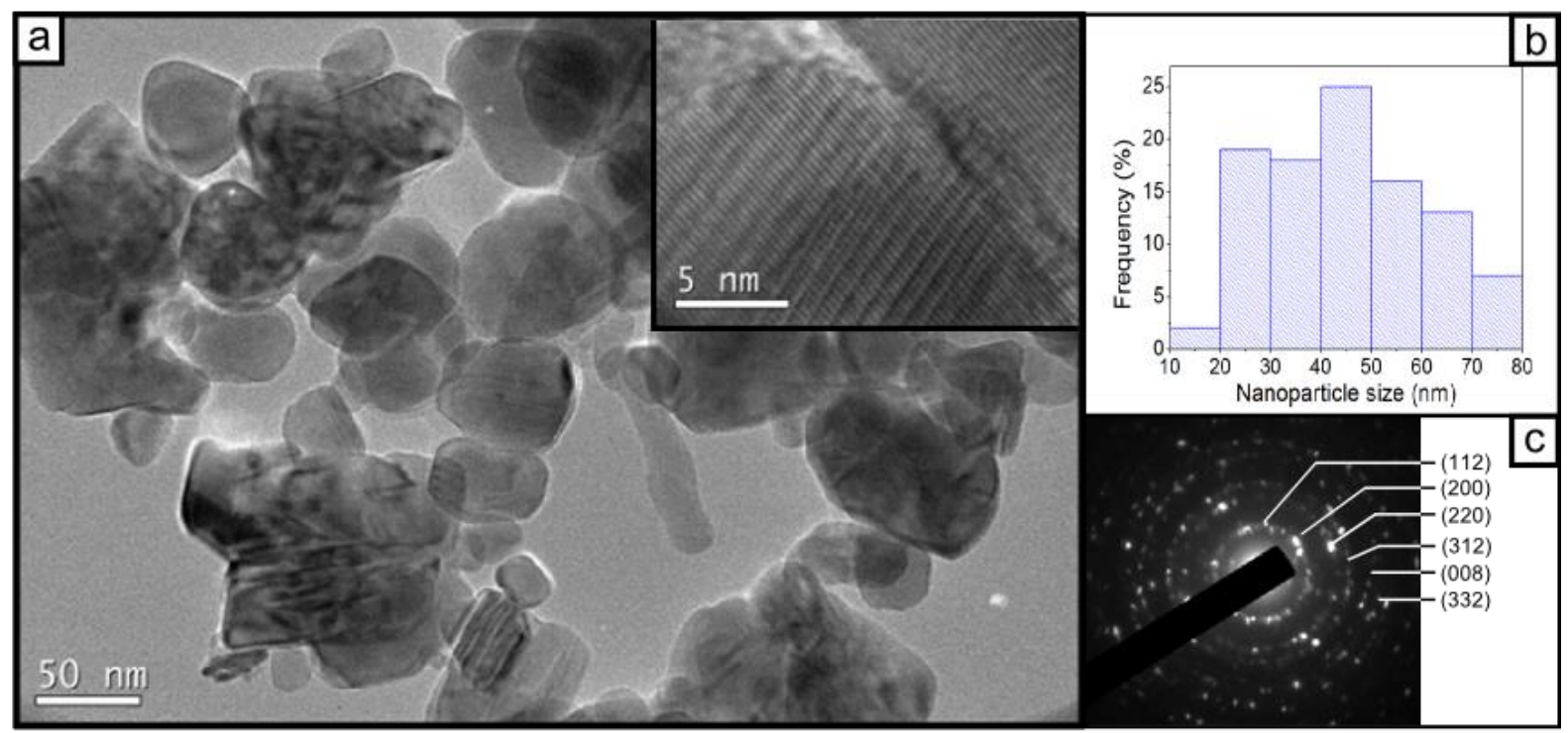

Figure 4.13: a)TEM image of nanocrystals obtained in experiment 7 with an inset of a HRTEM exhibiting the lattice fringes of one nanocrystal, asserting the monocrystalline nature of the CZTS nanoparticles. b) size distribution obtained from TEM images. c) represents the electron diffraction of the particles seen in a)

The nanocrystals present mostly faceted shapes. The average size is estimated at $45.2 \pm$ $31.4 \mathrm{~nm}$ which is larger than the similar method reported by Steinhagen et al. ${ }^{5}$ The high resolution TEM image of one nanocrystals presented in the inset of figure 4.13 a) shows lattice fringes. The distance between fringes is measured to be $0.31 \mathrm{~nm}$, which correspond to the (112) orientation of the kesterite CZTS structure. The average size of the nanoparticles is estimated at $43 \pm 36 \mathrm{~nm}$ by measuring over 200 nanoparticles from different areas of the TEM grid. The electron diffraction (fig $4.13 \mathrm{c}$ )) obtained for the particles in figure 4.13 a) present six distinct diffraction rings that matches the reflections of the CZTS crystal structure.

Using experiment 7 as a standard, we will be able to compare the particle size, shape and structure in experiments 8 and 9 and evidence the effect of the hydride reducing agent.

\section{Experiment 8: $\mathrm{LiBH}_{4}$ as reducing agent}

Experiment 8 explores the role of lithium borohydride injected in the reaction mixture before heating. 
Experimental

\begin{tabular}{|c|c|c|c|c|c|c|c|}
\hline $\begin{array}{c}\text { Exp } \\
\text { No }\end{array}$ & $\mathrm{Cu}$ & $\mathrm{Zn}$ & Sn & S & Temperature & Solvent & $\begin{array}{c}\text { Reducing } \\
\text { agent }\end{array}$ \\
\hline 8 & $\begin{array}{c}0.5 \\
\mathrm{mmol}\end{array}$ & $\begin{array}{c}0.3 \\
\mathrm{mmol}\end{array}$ & $\begin{array}{c}0.2 \\
\mathrm{mmol}\end{array}$ & $\begin{array}{c}1 \\
\mathrm{mmol}\end{array}$ & 280 & Oleylamine & $\mathrm{LiBH}_{4}$ \\
\hline
\end{tabular}

Table 4.6: Key variables in experiment 8

$0.099 \mathrm{~g}$ of copper acetate, $0.075 \mathrm{~g}$ of zinc acetate, $0.045 \mathrm{~g}$ of tin dichloride and $0.035 \mathrm{~g}$ of elemental sulfur were ground together in a mortar and suspended in $10 \mathrm{~mL}$ of oleylamine. The solution has a dark brown colour. The mixture is transferred into a 50 $\mathrm{mL}$ three necked flask linked to a distillation set-up. The mixture is degassed under vacuum for $1 \mathrm{~h}$ at room temperature then purged with nitrogen for 15 minutes. $10 \mathrm{~mL}$ of $2 \mathrm{M}$ lithium borohydride in tetrahydrofuran was injected in the solution. An large amount of foam was produced and some of the solution transferred into the collection flask of the distillation apparatus. After 15 minutes of reaction, $5 \mathrm{~mL}$ of methanol was added to the mixture to quench the remaining unreacted reducing agent. The temperature was increased to $130^{\circ} \mathrm{C}$ for 1 hour to remove the methanol and the tetrahydrofuran. The temperature is then raised to $280^{\circ} \mathrm{C}$ and the mixture was left to react for one hour. The system was slowly cooled to room temperature. $5 \mathrm{~mL}$ of ethanol was added to the mixture to facilitate the separation of the nanoparticles. The solid was collected by centrifugation at $140.000 \mathrm{rpm}$ for 2 minutes. The nanoparticles were washed 3 times in a mixture $1 / 1$ methanol/toluene.

\section{Results}

The crystal structure of the obtained crystal was determined by XRD. Figure 4.14 represents the spectrum for the nanocrystals synthesized in experiment 8 . The pattern is compared to the standard JCPDS card for CZTS. 


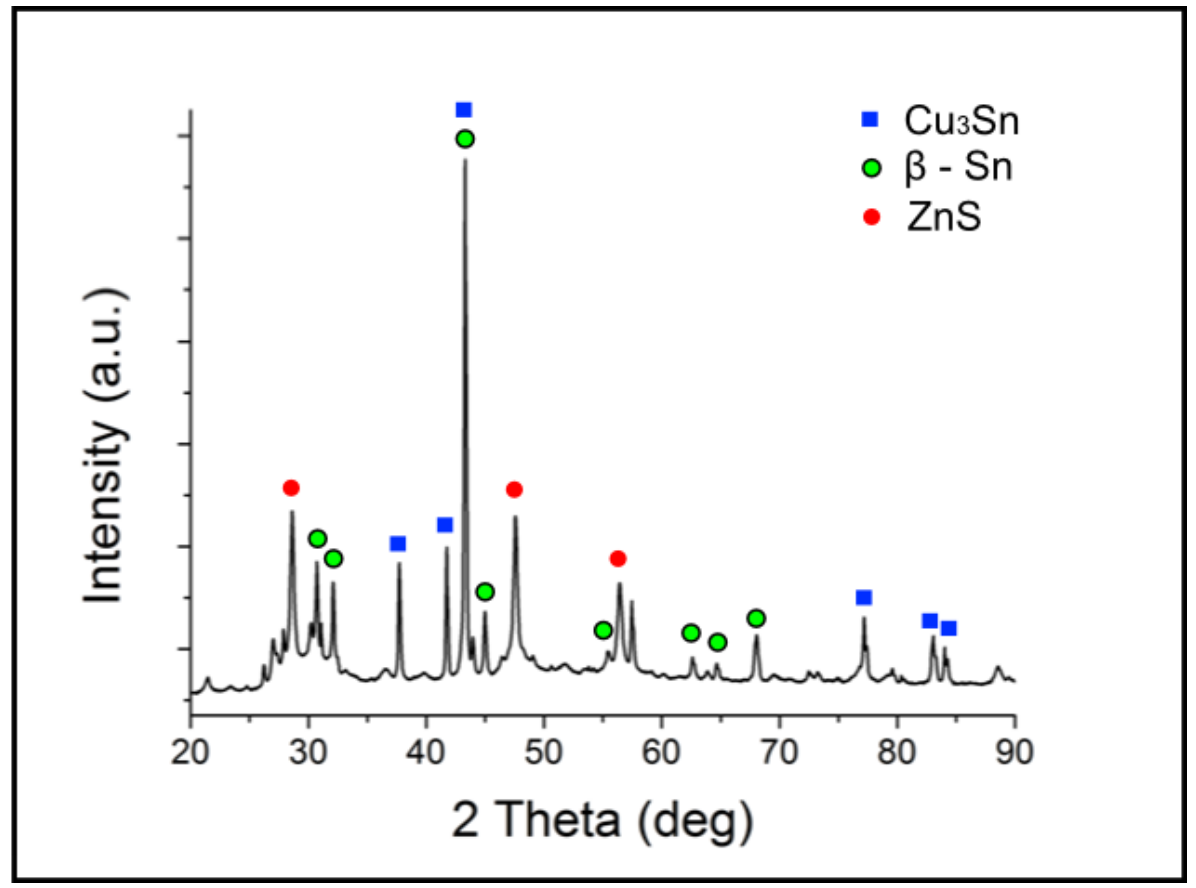

Figure 4.14: XRD pattern of the crystals obtained in experiment 8.

The XRD spectrum for nanoparticles obtained by chemical reduction with $\mathrm{LiBH}_{4}$ followed by a heat treatment at $280^{\circ} \mathrm{C}$ present multiple peaks, attesting of the crystalline nature of the nanoparticles. The spectrum does not match the JCPDS standard for CZTS. The spectrum can be matched to three structures: $\mathrm{Cu}_{3} \mathrm{Sn}$ (JCPDS card 01-1240), ZnS (JCPDS card 01-0792) and $\beta$-Sn (JCPDS card 86-2264). If all the precursors were decomposed at the same time, we expect the formation of small size CZTS crystals. The presence of these three different phases proves that the use of lithium borohydride did not narrow the decomposition time difference between the four precursors.

Using transmission electron microscopy, we can determine the size and shape of the nanocrystals and assess the effect of the $\mathrm{LiBH}_{4}$ reduction on the particle size distribution. Figure 4.15 represents the TEM images of the particles obtained in experiment 8 , the corresponding size distribution and the electron diffraction spectroscopy image of the crystals. 


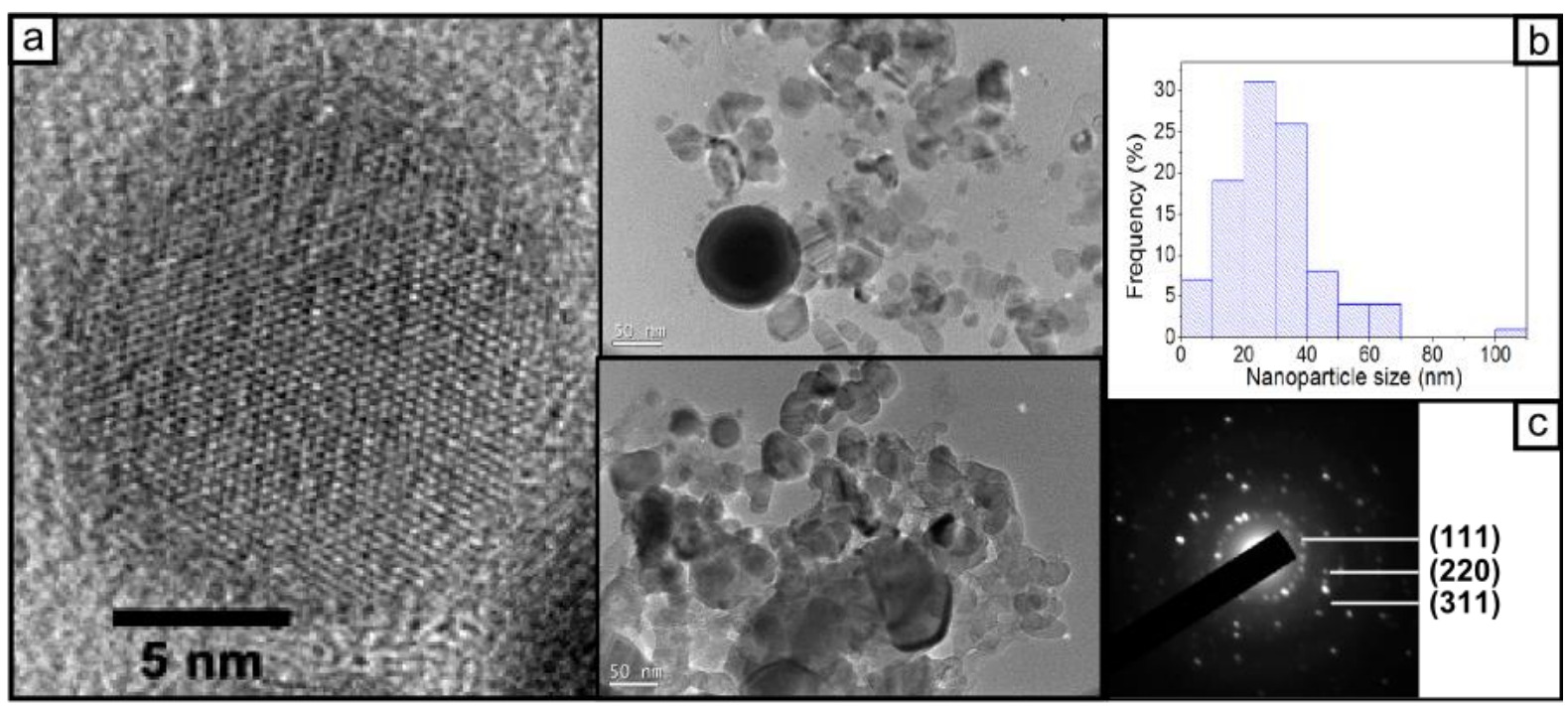

Figure 4.15: a) HRTEM and TEM images of crystals from experiment 8 and b) the corresponding size distribution. c) the EDS image exhibiting the diffraction rings.

Figure 4.15 a) shows TEM images of the nanocrystals obtained after reduction with $\mathrm{LiBH}_{4}$. The nanocrystals vary in size and shape. The HRTEM image have a $3.3 \AA$ spacing between lattice fringes that correspond to the (111) orientation of $\mathrm{ZnS} .^{28,29}$ The average size of the nanocrystals in estimated at $29.1 \pm 51.5 \mathrm{~nm}$. The particles are more disperse in size and larger than expected with the use of $\mathrm{LiBH}_{4}$. The EDS diffraction rings match mostly the ZnS structure, but lower intensity rings are present too, which is in good agreement with the XRD data of the nanocrystals. ${ }^{30}$

The addition of lithium borohydride led to the production of multiple phases, but not $\mathrm{Cu}_{2} \mathrm{SnZnS}_{4}$. The hydride reducing agent was expected to get closer to the "burst" nucleation phenomenon than the temperature decomposition, as the different precursors decompose at different temperature and the heating process is not instant enough to create a flash nucleation. $\mathrm{LiBH}_{4}$ was not strong enough to simultaneously decompose all the precursors. A stronger reducing agent like $\mathrm{LiAlH}_{4}$ might achieve a short decomposition event. 


\section{Experiment 9: $\mathrm{LiAlH}_{4}$ as reducing agent}

$\mathrm{LiBH}_{4}$ did not shorten the reduction time difference between the four precursors. $\mathrm{LiAlH}_{4}$ is a stronger reducing agent, so it is expected to quickly reduce the precursors into nuclei to form small particles of pure CZTS. Experiment 9 explores the role of lithium aluminium hydride injected in the reaction mixture before heating.

\section{Experimental}

\begin{tabular}{|c|c|c|c|c|c|c|c|}
\hline $\begin{array}{c}\text { Exp } \\
\text { No }\end{array}$ & Cu & Zn & Sn & S & Temperature & Solvent & $\begin{array}{c}\text { Reducing } \\
\text { agent }\end{array}$ \\
\hline 9 & $\begin{array}{c}0.5 \\
\mathrm{mmol}\end{array}$ & $\begin{array}{c}0.3 \\
\mathrm{mmol}\end{array}$ & $\begin{array}{c}0.2 \\
\mathrm{mmol}\end{array}$ & $\begin{array}{c}1 \\
\mathrm{mmol}\end{array}$ & 130 & Oleylamine & $\mathrm{LiAlH}_{4}$ \\
\hline
\end{tabular}

Table 4.7: Key variables in experiment 9

$0.099 \mathrm{~g}$ of copper acetate, $0.075 \mathrm{~g}$ of zinc acetate, $0.045 \mathrm{~g}$ of tin dichloride and $0.035 \mathrm{~g}$ of elemental sulfur were grinded together in a mortar and dissolved into $10 \mathrm{~mL}$ of oleylamine. The mixture is transferred into a $50 \mathrm{~mL}$ three necked flask linked to a distillation set-up. The mixture is degassed under vacuum for 3 hours at room temperature. The mixture was then slowly heated up $130^{\circ} \mathrm{C}$ under vacuum for 3 more hours, until the bubbling of the oleylamine stopped, indicating the removal of all the water impurity in the oleylamine and the water contained in the precursors. The mixture was let to cool down naturally and purged with nitrogen over night. $10 \mathrm{~mL}$ of lithium aluminium hydride were supposed to be injected in the mixture, but the injection of around $1 \mathrm{~mL}$ provoked a violent gas reaction, the reaction was aborted.

To prevent the violent reaction created by the introduction of $\mathrm{LiAlH}_{4}$, the next experiments (10 and 11) will be conducted in a larger volume of solvent.

\section{Experiments 10 and 11: using lower precursors concentrations}

Experiments 10 and 11 explore the function of the reducing agents lithium borohydride and lithium aluminium hydride in a larger amount of solvent to precursor ratio to avoid the violent reaction created by the injection of the strong hydride reducing agents. 
Experiment 10: $\mathrm{LiBH}_{4}$ as reducing agent in a lower concentrated solution than $\exp 8$

Experiment 10 looks into the role of lithium borohydride injected in the reaction mixture containing a larger amount of solvent than experiment 9 before heating.

\section{Experimental}

\begin{tabular}{|c|c|c|c|c|c|c|c|}
\hline $\begin{array}{c}\text { Exp } \\
\text { No }\end{array}$ & $\mathbf{C u}$ & Zn & Sn & S & Temperature & Solvent & $\begin{array}{c}\text { Reducing } \\
\text { agent }\end{array}$ \\
\hline 10 & $\begin{array}{c}0.1 \\
\mathrm{mmol}\end{array}$ & $\begin{array}{c}0.06 \\
\mathrm{mmol}\end{array}$ & $\begin{array}{c}0.04 \\
\mathrm{mmol}\end{array}$ & $\begin{array}{c}0.2 \\
\mathrm{mmol}\end{array}$ & 280 & $\begin{array}{c}\text { Oleylamine } \\
\text { and } \\
\text { Octadecene }\end{array}$ & $\mathrm{LiBH}_{4}$ \\
\hline
\end{tabular}

Table 4.7: Key variables in experiment 10

$0.0198 \mathrm{~g}$ of copper acetate, $0.015 \mathrm{~g}$ of zinc acetate, $0.009 \mathrm{~g}$ of tin dichloride and $0.007 \mathrm{~g}$ of elemental sulfur were grinded together in a mortar and dissolved into $10 \mathrm{~mL}$ of oleylamine and transferred into a $100 \mathrm{~mL}$ three necked flask attached to a distillation set-up. The mixture was degassed under vacuum for two hours at room temperature. The temperature was raised to $100^{\circ} \mathrm{C}$ under vacuum for one hour. The mixture was then purged with nitrogen and cooled to room temperature. $20 \mathrm{~mL}$ of octadecene was injected in the reaction vessel and degassed under vaccum for another 30 minutes. $2 \mathrm{~mL}$ of lithium borohydride were injected into the flask and left to react for 10 minutes. The excess $\mathrm{LiAlH}_{4}$ was quenched by the injection of $2 \mathrm{~mL}$ of methanol. The temperature is then raised to $280^{\circ} \mathrm{C}$ and the mixture was left to react for one hour. The system was slowly cooled to room temperature. $5 \mathrm{~mL}$ of ethanol was added to the mixture to facilitate the separation of the nanoparticles. The solid was collected by centrifugation at $140.000 \mathrm{rpm}$ for 2 minutes. The nanoparticles were washed 3 times in a mixture 1/1 methanol/toluene.

\section{Results}

The XRD analysis of the nanocrystals obtained after reduction with $\mathrm{LiBH}_{4}$ is presented in figure 4.16. We expect the peak position and intensity to match the JCPDS standard for $\mathrm{Cu}_{2} \mathrm{ZnSnS}_{4}$. 


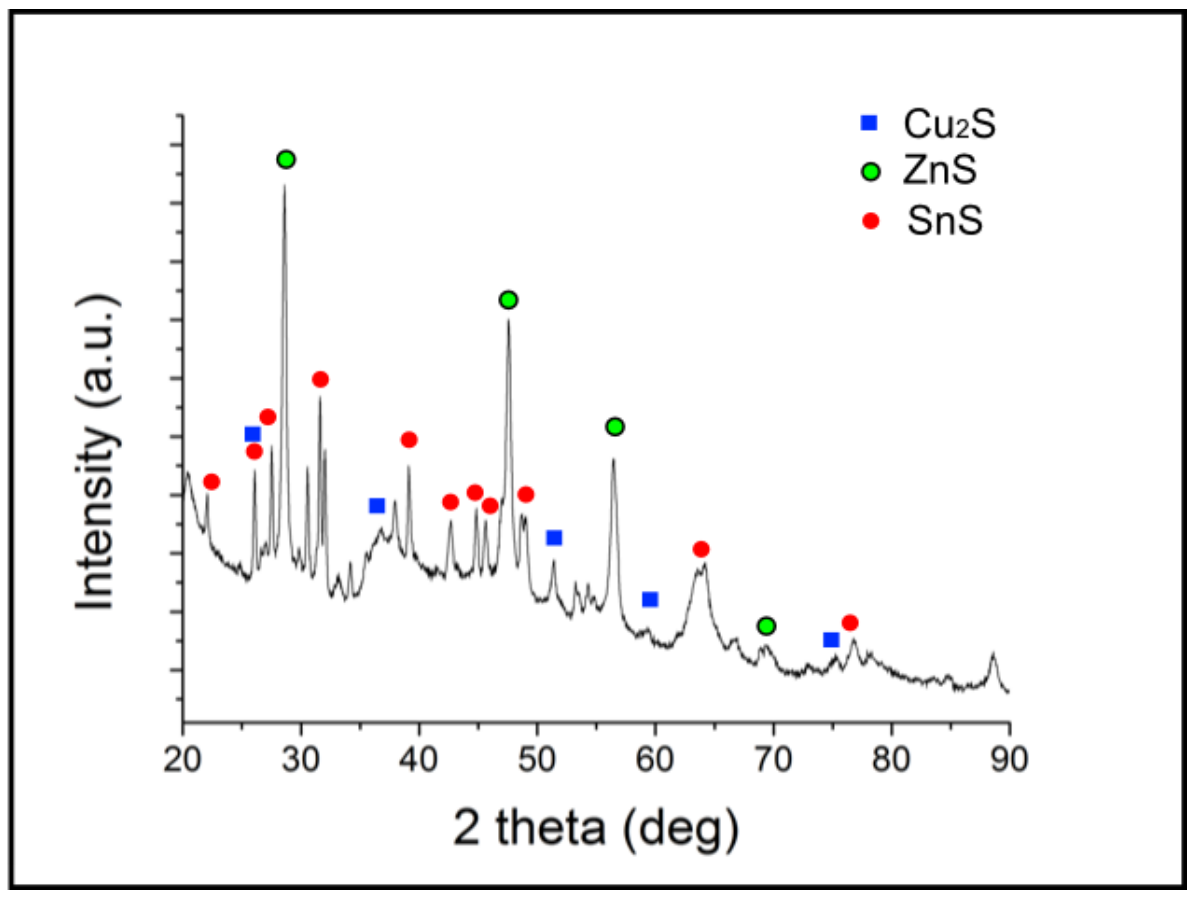

Figure 4.16: XRD spectrum from nanocrystals in experiment 10.

The spectrum in figure 4.16 does not match the CZTS structure, but presents a multitude of peaks assessing of the crystalline nature of the nanoparticles. The peaks can be matched to mostly ZnS (JCPDS card 01-0792), SnS (JCPDS card 01-0984) and $\mathrm{Cu}_{2} \mathrm{~S}$ (JCPDS card 46-1195).

TEM imaging will give us the average size and shape as the particles and SAED will evidence the structure of nanocrystals of a given area of the TEM grid. (fig 4.17)

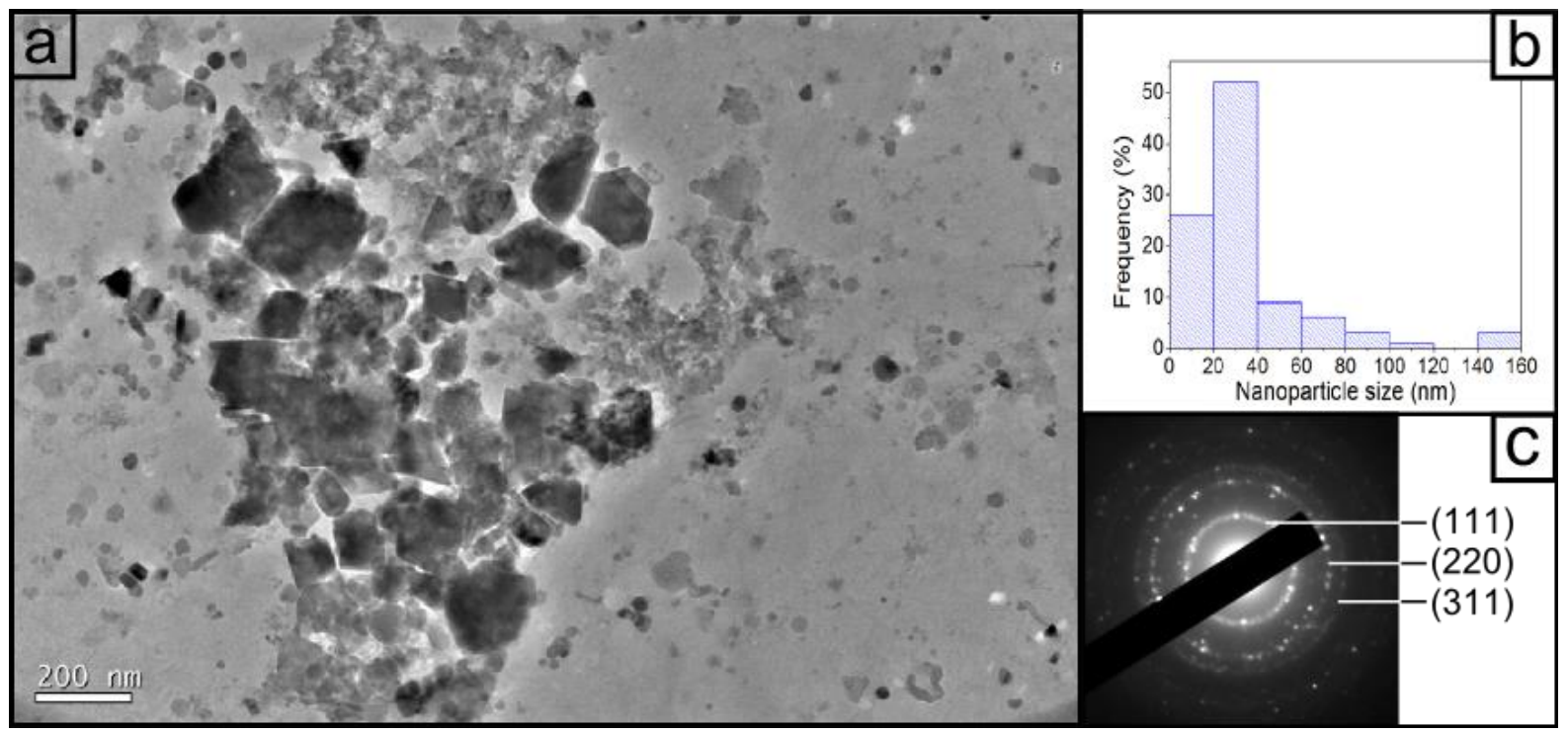


Figure 4.17: a)TEM image of nanocrystal from experiment 10, b) the corresponding size distribution and c) the electron diffraction rings from the nanocrystals in a).

The nanocrystals vary in size and shape, from small spherical crystals to large hexagonal crystals. The average size of the nanoparticles is estimated at $35.4 \pm 70.4 \mathrm{~nm}$. The EDS diffraction rings match mostly the ZnS crystal structure.

The introduction of lithium borohydride did not succeed in the production of small monodisperse CZTS nanocrystals. Using $\mathrm{LiBH}_{4}$ generated a large size distribution and a multitude of crystal structure. As lithium aluminium hydride has stronger reducing properties, we expect to reach these goals in experiment 11.

Experiment 11: $\mathrm{LiBH}_{4}$ as reducing agent in a lower concentrated solution than $\exp 9$

In experiment 11, the role of the reduction of the zinc, copper and tin precursors using lithium aluminium hydride before the heating process is assessed.

\section{Experimental}

\begin{tabular}{|c|c|c|c|c|c|c|c|}
\hline $\begin{array}{c}\text { Exp } \\
\text { No }\end{array}$ & $\mathrm{Cu}$ & $\mathrm{Zn}$ & $\mathrm{Sn}$ & $\mathbf{S}$ & Temperature & Solvent & $\begin{array}{c}\text { Reducing } \\
\text { agent }\end{array}$ \\
\hline 11 & $\begin{array}{c}0.1 \\
\mathrm{mmol}\end{array}$ & $\begin{array}{c}0.06 \\
\mathrm{mmol}\end{array}$ & $\begin{array}{c}0.04 \\
\mathrm{mmol}\end{array}$ & $\begin{array}{c}0.2 \\
\mathrm{mmol}\end{array}$ & 280 & $\begin{array}{c}\text { Oleylamine and } \\
\text { Octadecene }\end{array}$ & $\mathrm{LiAlH}_{4}$ \\
\hline
\end{tabular}

Table 4.9: Key variables in experiment 11

$0.0198 \mathrm{~g}$ of copper acetate, $0.006 \mathrm{~g}$ of zinc acetate, $0.009 \mathrm{~g}$ of tin dichloride and $0.007 \mathrm{~g}$ of elemental sulfur were grinded together in a mortar and dissolved into $10 \mathrm{~mL}$ of oleylamine and $20 \mathrm{~mL}$ of octadecene and transferred into a $100 \mathrm{~mL}$ three necked flask attached to a distillation set-up. After 30 minutes of degassement at room temperature, the temperature was raised to $110^{\circ} \mathrm{C}$ and the mixture was kept under vacuum for four hours. The mixture was then purged under nitrogen and cooled down to room temperature naturally. $2 \mathrm{~mL}$ of lithium aluminium hydride was injected in the flask. A large amount of foam was produced, and some of the reaction mixture transferred into the collection flask of the distillation apparatus. The remaining reducing agent was quenched with $2 \mathrm{~mL}$ of methanol. The mixture was heated to $130^{\circ} \mathrm{C}$ for one hour to remove the low boiling point solvents present in the mixture (methanol and 
tetrahydrofuran). The temperature was raised to $280^{\circ} \mathrm{C}$ and the solution was left to react for one hour. After the reaction mixture was cooled down to room temperature naturally, $5 \mathrm{~mL}$ of ethanol was injected to facilitate the separation of the crystals. After centrifugation at $140.000 \mathrm{rpm}$ for 5 minutes followed by three washings in a $1 / 1$ mixture of methanol and toluene, no solid was collected. The produce of the reaction was some form of gel. The reaction was aborted.

The combination of heat treatment and chemical reduction by hydride reducing agent produces violent reactions that are difficult to control. A room temperature approach for chemical reduction is preferable. In the next section, we introduce the reverse micelle technique for CZTS nanocrystals synthesis, involving the chemical reduction of precursors in room temperature solvent in presence of a surfactant molecule.

\subsubsection{Room temperature synthesis by chemical reduction based on the reverse micelle principle}

The high temperature syntheses reported previously in this chapter did not achieve a monodisperse small growth of CZTS nanocrystals. In this section, the reverse micelle synthetic method to produce CZTS nanocrystals was investigated. The surfactant/solvent mixture combined to the use of a strong reducing agent is expected to synthesize small monodisperse CZTS particles. Chemical reduction synthesis in reverse microemulsion has been first developed to produce metallic nanoparticles. ${ }^{31}$ In the Tilley group, the reverse micelle synthesis was developed to produce small monodisperse silicon and germanium nanoparticles. ${ }^{32-33}$

In the following experiments (12-14), the syntheses were conducted at room temperature, the reaction being initiated by the reduction of metal salts into monomers using strong hydride reducing agents.

\section{Experiment 12 : reverse micelle synthesis and reduction using $\mathrm{LiAlH}_{4}$}

Experiment 12 describes the reverse micelle synthesis using lithium aluminium hydride as a reducing agent. 
Experimental

\begin{tabular}{|c|c|c|c|c|c|c|c|c|}
\hline $\begin{array}{c}\text { Exp } \\
\text { No }\end{array}$ & $\mathbf{C u}$ & $\mathbf{Z n}$ & Sn & S & Temperature & Solvent & Surfactant & $\begin{array}{c}\text { Reducing } \\
\text { agent }\end{array}$ \\
\hline 12 & $\begin{array}{c}0.05 \\
\mathrm{mmol}\end{array}$ & $\begin{array}{c}0.025 \\
\mathrm{mmol}\end{array}$ & $\begin{array}{c}0.025 \\
\mathrm{mmol}\end{array}$ & $\begin{array}{c}0.1 \\
\mathrm{mmol}\end{array}$ & $\begin{array}{c}\text { Room } \\
\text { temperature }\end{array}$ & Toluene & TOAB & $\mathrm{LiAlH}_{4}$ \\
\hline
\end{tabular}

Table 4.10: Key variables in experiment 12

$0.0099 \mathrm{~g}$ of copper acetate, $0.008 \mathrm{~g}$ of zinc acetate, $0.005 \mathrm{~g}$ of tin dichloride, $0.004 \mathrm{~g}$ of elemental sulfur and $0.5 \mathrm{~g}$ of tetraoctylammonium bromide were ground together in a mortar. $5 \mathrm{~mL}$ of toluene was added. The solution was transferred into a Schlenk tube and degassed under vacuum for 15 minutes. About half of the toluene was evaporated and collected in a cold trap. After purging with nitrogen for 10 minutes, $30 \mathrm{~mL}$ of anhydrous toluene was injected in the Schlenk tube. The mixture was purged with nitrogen for another thirty minutes. $3 \mathrm{~mL}$ of $1 \mathrm{M}$ lithium aluminium hydride solution in THF were then swiftly injected in the mixture. The blue-green solution turns orange then black rapidly. After 30 minutes, $5 \mathrm{~mL}$ of ethanol was added to the mixture to quench the excess $\mathrm{LiAlH}_{4}$. Some foam is produced. The particles were separated by centrifugation at $140.000 \mathrm{rpm}$ for 5 minutes followed by three washings in a $1 / 1$ mixture of methanol and toluene.

\section{Results}

The nanocrystals were first analysed by XRD to determine their crystal structure.

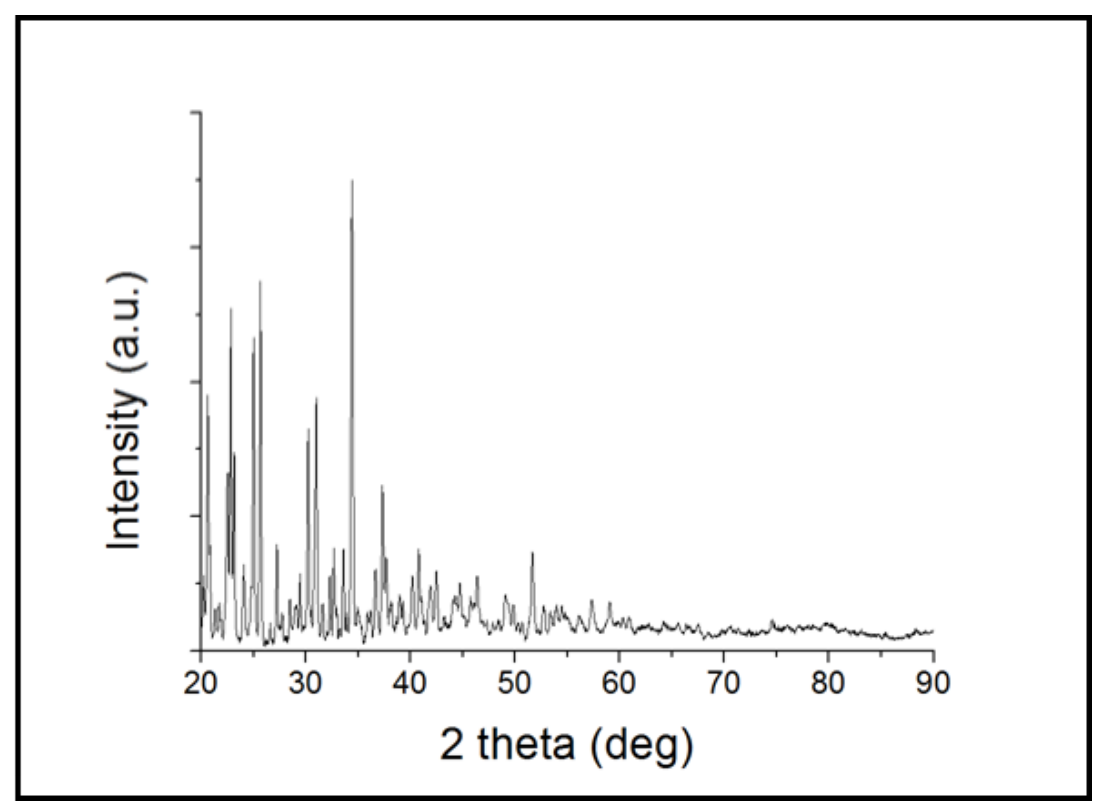

Figure 4.18: XRD spectrum of the nanoparticles obtained in experiment 12 
The XRD spectrum obtained after the microemulsion synthesis using $\mathrm{LiAlH}_{4}$ presents a multitude of crystals phases. The largest peaks can be matched to $\mathrm{Cu}_{2} \mathrm{~S}$ and $\mathrm{Al}_{2} \mathrm{~S}_{3}$. Alumina can match some of the peaks. But other impurity structures are also present. It is difficult to assess the exact composition of the nanocrystals as many of the XRD patterns possible with the element present in solution present similar peak positions.

TEM imaging provides information towards the size and shape of the formed nanocrystals. Figure 5.9 is a TEM image of the particles obtained in experiment 12.

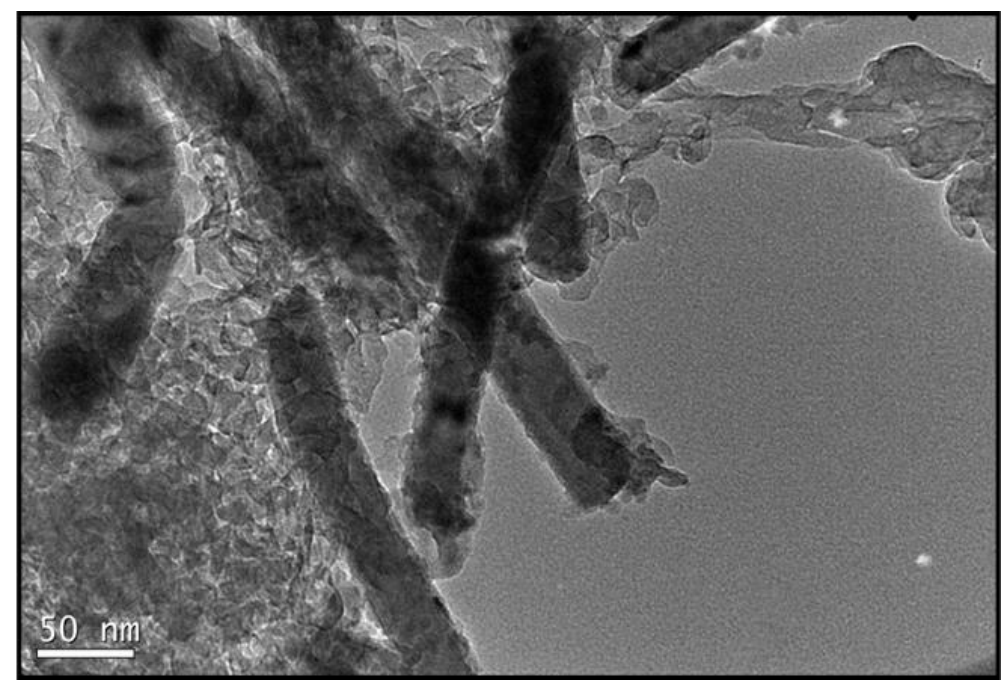

Figure 4.19: Low resolution TEM image of the crystals obtained in experiment 12

The TEM image show large nanowires about $250 \mathrm{~nm}$ long and $40 \mathrm{~nm}$ wide. The rest of the matter seen on the image does not have a particle shape. It is most likely agglomerated combinations of the reactants. According to the literature, CZTS has not been synthesized in the nanowire shape without a hard template like porous alumina (AAO). ${ }^{34-35}$

The nature and composition of the nanowires and other particles observed on the TEM image can be determined by electron dispersion spectroscopy (EDS) by determining the ratio of each element present in the sample. Figure 4.20 is the EDS spectrum obtained from the particles obtained in experiment 12 . 


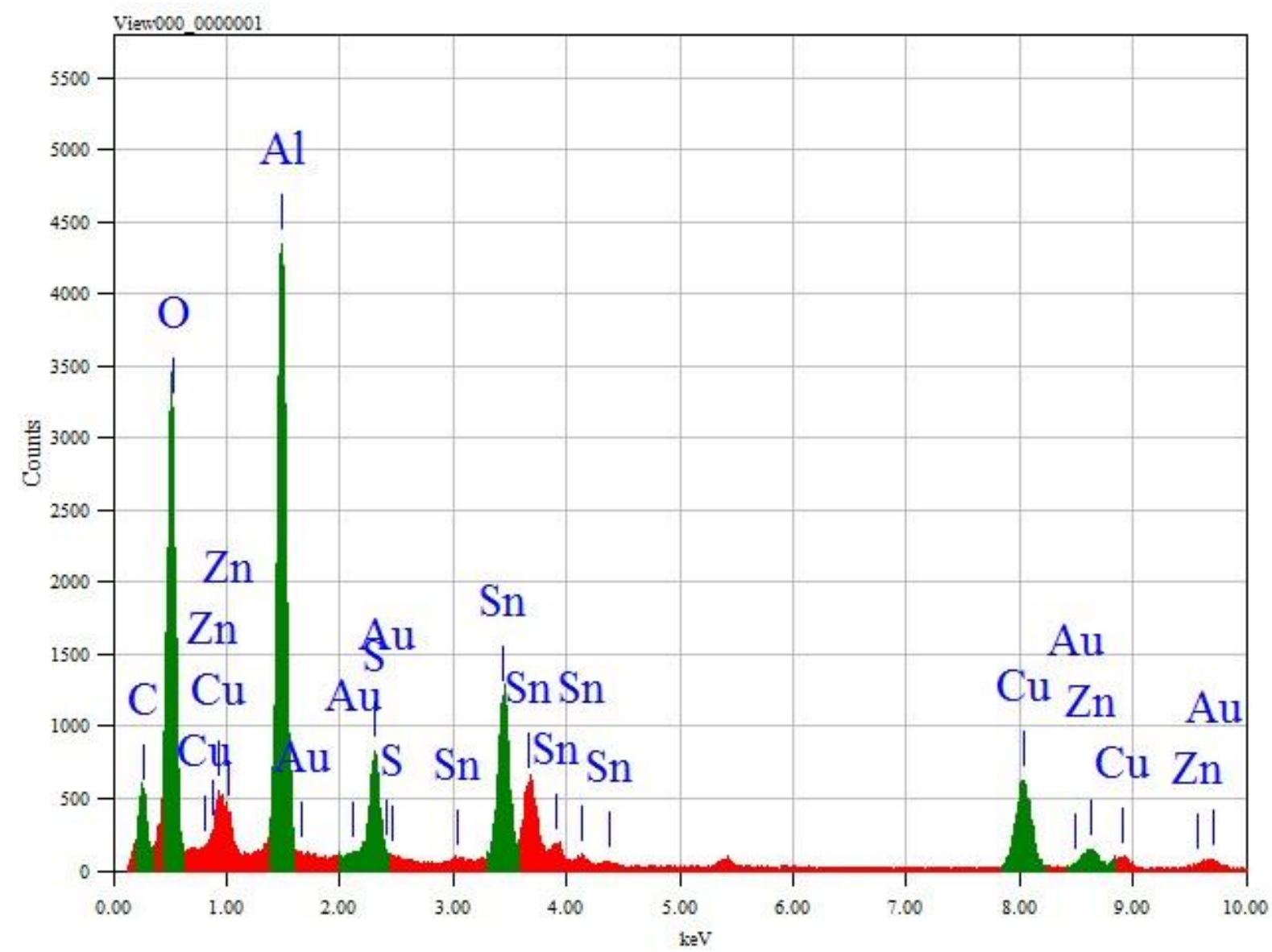

Figure 4.20: EDS spectra obtained for the crystals synthesized by the reduction of $\mathrm{LiAlH}_{4}$ in toluene at room temperature

EDS analysis gives the following ratio of elements: carbon $18 \%$, oxygen $43 \%$, aluminium $22 \%$, sulphur $4 \%$, copper $5 \%$, zinc $1 \%$ and tin $7 \%$. The gold peak correspond to the gold from the TEM grid. The high ratio of oxygen and aluminium suggest that the nanowires observed on the TEM image are composed of alumina. Alumina can be grown in the nanowire configuration. ${ }^{36,37}$ The quenching of the large excess $\mathrm{LiAlH}_{4}$ with methanol lead to the production of those alumina crystals and they were not seggregated from the particles made by the copper, zinc, tin and sulphur during the purification process.

Using a different reducing agent, such as $\mathrm{LiBH}_{4}$, should remove alumina or other aluminium impurities. 


\section{Experiment 13: reverse micelle synthesis and reduction using $\mathrm{LiBH}_{4}$}

This experiment explores the synthesis at room temperature using the molecular ratios as followed:

$$
\mathrm{Cu}:(\mathrm{Zn}+\mathrm{Sn})=1 \text { and } \mathrm{Zn}: \mathrm{Sn}=1
$$

It is the same ratio of precursors as used in experiment 13. The difference between experiments 12 and 13 rely on the reducing agent. In this experiment, we observe the role played by $\mathrm{LiBH}_{4}$ and expect to avoid the production of alumina impurities like in experiment 12 .

Experimental

\begin{tabular}{|c|c|c|c|c|c|c|c|c|}
\hline $\begin{array}{c}\text { Exp } \\
\text { No }\end{array}$ & Cu & Zn & Sn & S & Temperature & Solvent & Surfactant & $\begin{array}{c}\text { Reducing } \\
\text { agent }\end{array}$ \\
\hline 13 & $\begin{array}{c}0.5 \\
\mathrm{mmol}\end{array}$ & $\begin{array}{c}0.25 \\
\mathrm{mmol}\end{array}$ & $\begin{array}{c}0.25 \\
\mathrm{mmol}\end{array}$ & $\begin{array}{c}1 \\
\mathrm{mmol}\end{array}$ & $\begin{array}{c}\text { Room } \\
\text { temperature }\end{array}$ & Toluene & $\mathrm{TOAB}$ & $\mathrm{LiBH}_{4}$ \\
\hline
\end{tabular}

Table 4.11: Key variables in experiment 13

$0.5 \mathrm{~g}$ of TOAB and $0.085 \mathrm{~g}$ of copper chloride dihydrate were placed in a Schlenk tube, degassed under vacuum for 15 minutes then purged with nitrogen for 20 minutes. 30 $\mathrm{mL}$ of anhydrous toluene was injected into the tube. After 10 minutes of active stirring, $0.25 \mathrm{~mL}$ of zinc chloride solution and $0.25 \mathrm{~mL}$ of tin tetrachloride solution were added to the mixture. The solution present a deep red color. After 30 minutes of stirring, $4 \mathrm{~mL}$ of lithium borohydride solution in THF was injected in the reaction solution. The solution turns black. After 10 minutes, $0.032 \mathrm{~g}$ of sulphur dissolved into $2 \mathrm{~mL}$ of toluene were added to the mixture. The nanoparticles were collected by centrifugation at 140.000 rpm for 5 minutes and purified by three washings in a $1 / 1$ mixture of methanol and toluene.

\section{Results}

We first want to determine the crystal structure of the nanocrystals obtained in experiment 13 using XRD. Figure 5.21 represents the XRD spectrum. 


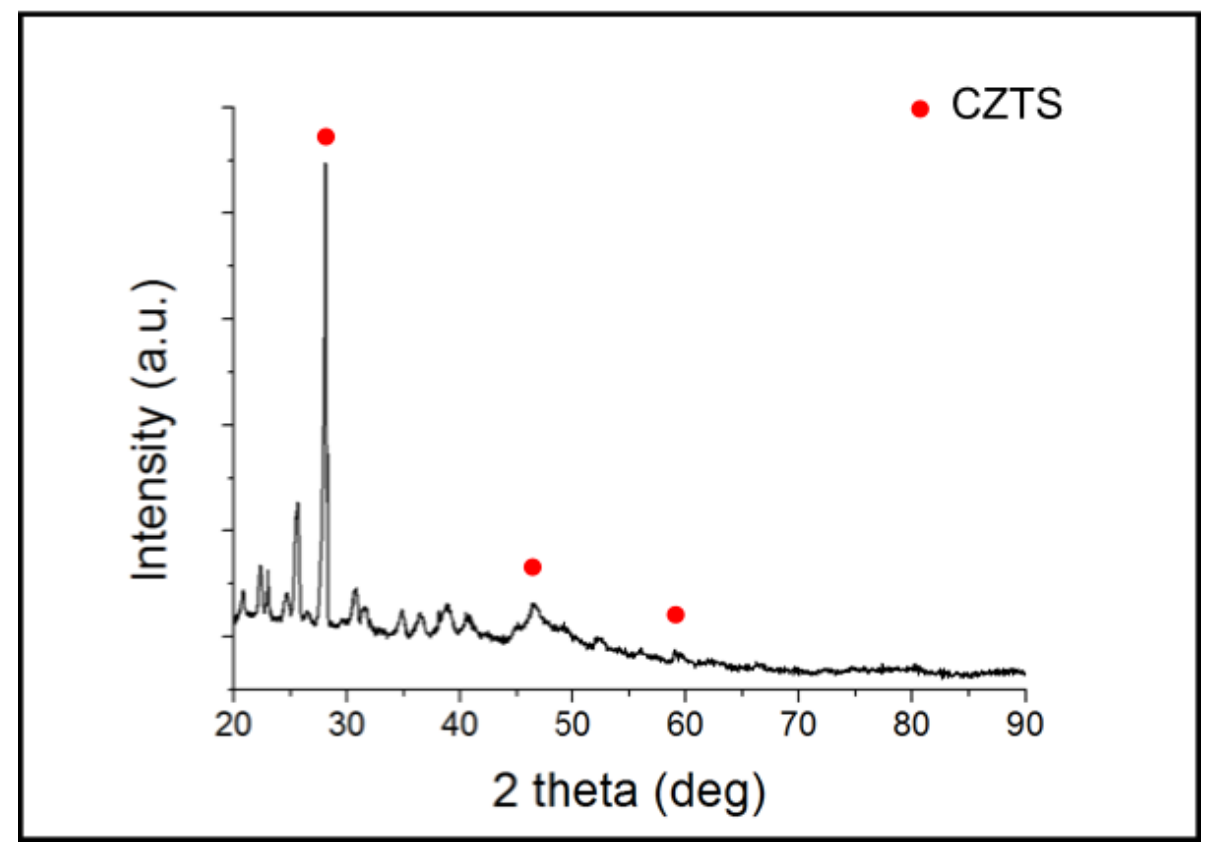

Figure 4.21: XRD spectrum from particles obtained in experiment 13

The XRD spectrum from particles synthesized using $\mathrm{LiBH}_{4}$ present several crystal structures. The main structure corresponds to the kesterite structure of CZTS. The impurity phases can be matched mostly to other sulphides such as $\mathrm{SnS}$ and $\mathrm{Cu}_{2} \mathrm{~S}$.

Using the transmission electron microscopy, we determine the size and shape of the nanocrystals. Also, SAED rings assess the structure of the crystals on a given area of the grid.

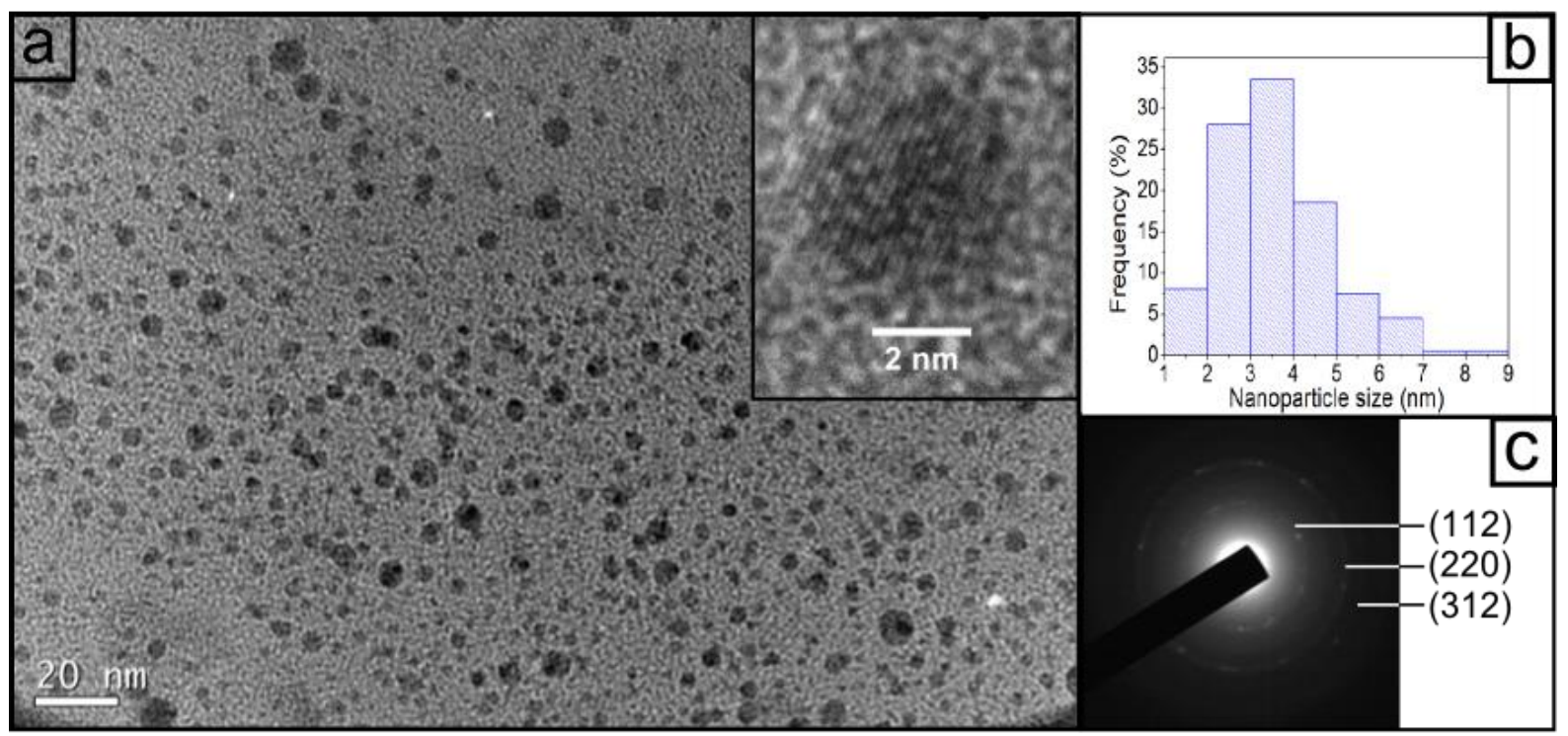

Figure 4.22: Low resolution TEM of the nanocrystals obtained in experiment 13, assessing the size and shape of the nanocrystals 
The particles have a spherical shape. The average size of the crystals is estimated at 3.8 $\pm 3.9 \mathrm{~nm}$ by measuring over 200 particles from different areas of the TEM grid. The nanoparticles are smaller than most reported CZTS particles. ${ }^{38-40}$ The $0.3 \mathrm{~nm}$ distance between lattice fringes on the high resolution TEM image seen in the inset of fig. 4.22 a) is in agreement with the distance between (112) crystal plans in CZTS nanostructures. The diffraction rings obtained by SAED measurements over the area of the grid represented in fig. 4.22 a) exhibit the 3 rings characteristic of CZTS structure.

According to the XRD data, the nanoparticles presented several crystal structures. TEM image in figure 4.22 only represents a small area of the grid. Performing EDS measurements on several areas of the grid will establish the nature of the crystals. Figure 4.23 represent the EDS spectrum of the specific area seen in figure 4.22 , but table 5.11 synthesises the molar percentages of each element for four different areas of the grid.

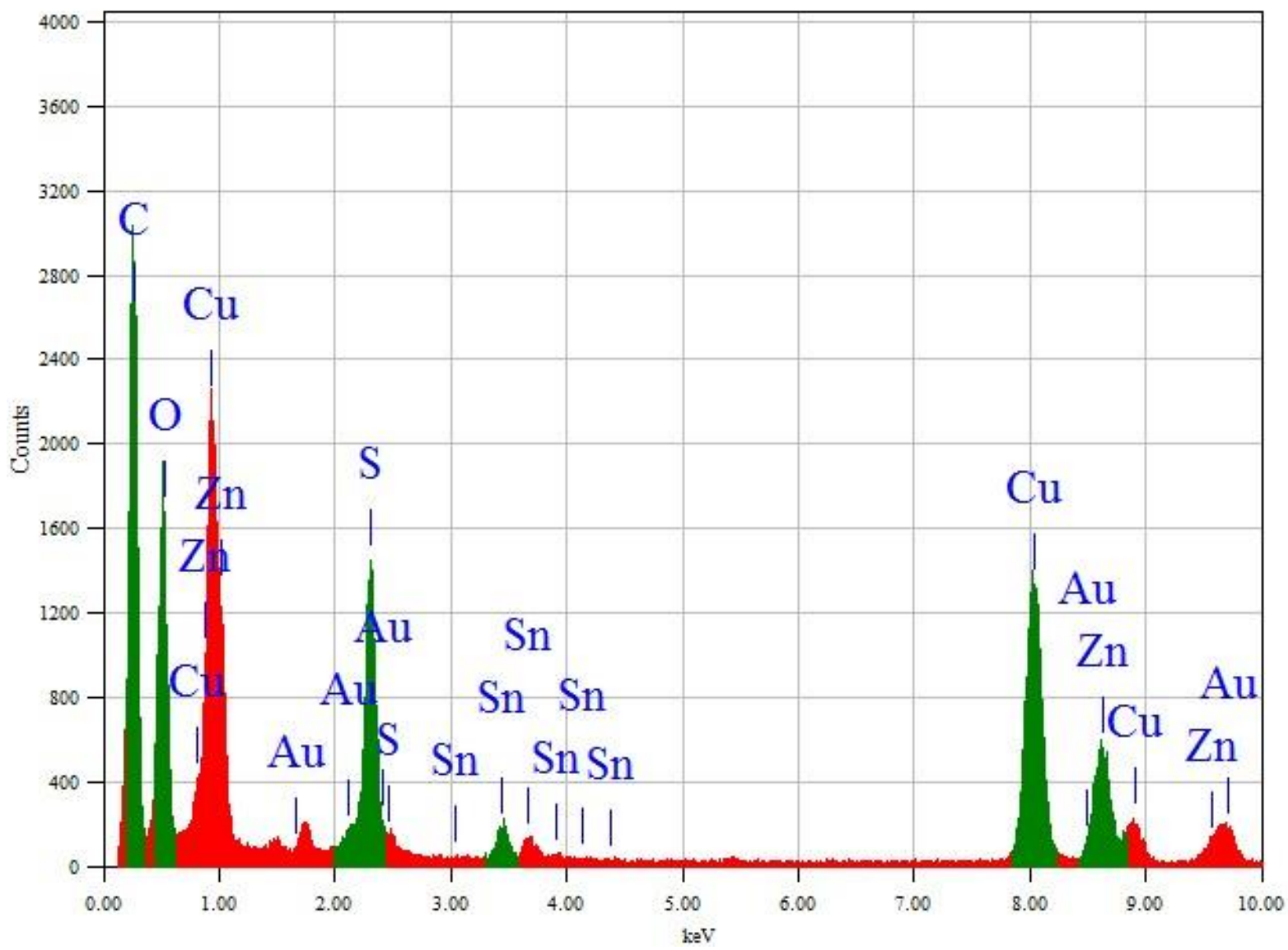

Figure 4.23: EDS pattern obtained for the area of nanocrystals shown in figure 5.22 


\begin{tabular}{|c|c|c|c|c|c|c|}
\hline $\begin{array}{c}\text { EDS } \\
\text { zone }\end{array}$ & $\mathbf{C u}$ & $\mathbf{Z n}$ & Sn & $\mathbf{S}$ & $\mathbf{C u}:(\mathbf{Z n}+\mathbf{S n})$ & $\mathbf{Z n}: \mathbf{S n}$ \\
\hline 1 & 13.07 & 4.52 & 1.39 & 8.69 & 2.21 & 3.25 \\
\hline 2 & 6.03 & 2.63 & 0.61 & 4.41 & 1.86 & 4.31 \\
\hline 3 & 4.22 & 0.99 & 0.21 & 3.04 & 3.52 & 4.71 \\
\hline 4 & 2.04 & 0.48 & 0.11 & 1.63 & 3.46 & 4.36 \\
\hline
\end{tabular}

Table 4.12: molar percentages of each elements and ratios calculated from EDS spectra for 4 different regions of nanocrystals obtained from experiment 13

The molar percentage of copper, tin, zinc and sulphur differ between the four different areas analysed. Also, none of the ratios match the initial ratio of the precursors in solution. Even if the SAED spectrum in figure 4.22 present the rings for CZTS nanocrystals, the molar ratios of all the elements in zone 1 does not allow to conclude to a successful CZTS nanocrystals synthesis. Other phases are present as impurities.

Using a different ratio of initial precursors is expected to limit the production of impurity phases seen in this experiment.

\section{Experiment 14: reverse micelle synthesis and reduction using $\mathrm{LiBH}_{4}$ with a tin}

\section{rich precursors ratios}

Due to the impurities phases observed in experiment 13, we expect a change of precursors relative ratio will limit the synthesis to only the CZTS crystal structure. The ratios of precursors in this experiment were calculated according to the proportions used in the synthesis published by the Prieto group and following these equations: 9,41

$$
\mathrm{Cu}:(\mathrm{Zn}+\mathrm{Sn})=0.8 \text { and } \mathrm{Zn}: \mathrm{Sn}=1.2
$$

The reducing agent used is $\mathrm{LiBH}_{4}$ in order to avoid aluminium impurities, like the ones observed in experiment 11. 
Experimental

\begin{tabular}{|c|c|c|c|c|c|c|c|}
\hline $\mathbf{C u}$ & $\mathbf{Z n}$ & Sn & S & Temperature & Solvent & Surfactant & $\begin{array}{c}\text { Reducing } \\
\text { agent }\end{array}$ \\
\hline $\begin{array}{c}0.44 \\
\mathrm{mmol}\end{array}$ & $\begin{array}{c}0.3 \\
\mathrm{mmol}\end{array}$ & $\begin{array}{c}0.25 \\
\mathrm{mmol}\end{array}$ & $\begin{array}{c}1 \\
\mathrm{mmol}\end{array}$ & $\begin{array}{c}\text { Room } \\
\text { temperature }\end{array}$ & Toluene & TOAB & $\mathrm{LiBH}_{4}$ \\
\hline
\end{tabular}

Table 4.13: Key variables in experiment 14

$0.5 \mathrm{~g}$ of copper chloride dihydrate and $0.5 \mathrm{~g}$ of TOAB were placed in a Schlenk tube. The reaction vessel was degassed under vacuum for 15 minutes then purged under nitrogen for twenty minutes. $30 \mathrm{~mL}$ of anhydrous toluene was injected. $0.3 \mathrm{~mL}$ of zinc chloride solution and $0.25 \mathrm{~mL}$ of tin tetrachloride solution were injected into the Schlenk tube. The dark brown solution was stirred for 15 minutes. $4 \mathrm{~mL}$ of $2 \mathrm{M}$ lithium borohydride in tetrahydrofuran was injected. The solution turned transparent instantly then black within a few seconds. After 30 minutes, $0.0032 \mathrm{~g}$ of sulphur dissolved into $2 \mathrm{~mL}$ of toluene was injected into the solution. The nanoparticles were collected by centrifugation at $140.000 \mathrm{rpm}$ for 5 minutes and purified by three washings in a $1 / 1$ mixture of methanol and toluene.

\section{Results}

We first assess the crystal structure of the nanocrystals via XRD. Figure 4.24 represents the XRD patter obtained for the crystals in experiment 14.

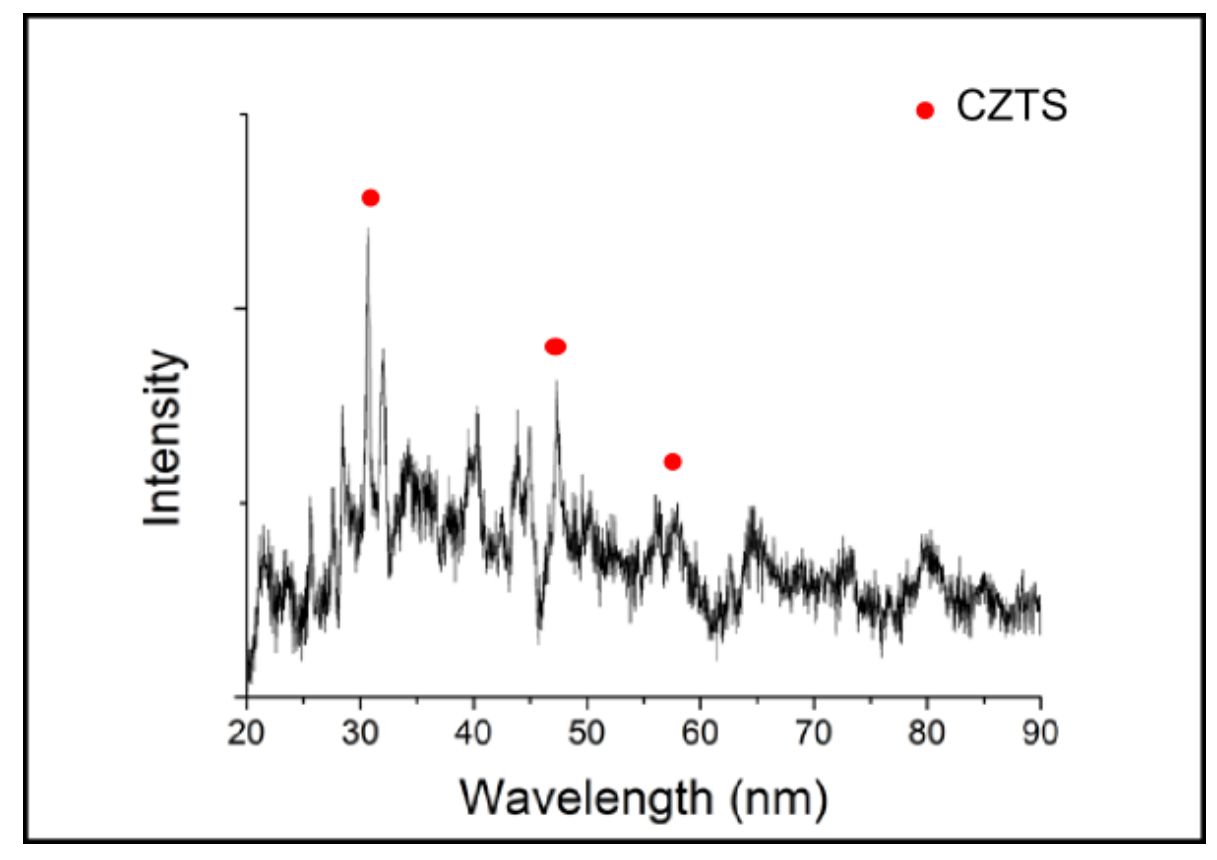

Figure 4.24: XRD pattern obtained from nanocrystals synthesized in experiment 14 
A large background noise was substracted from the XRD spectrum to clarify the diffraction peak pattern. The background noise arises from amorphous matter. Even after purification, the sample presents some amorphous material. The remaining peaks can be partially matched to the CZTS kesterite structure, as represented with red circles in figure 4.24 .

TEM images and selected area electron diffraction gives insight on the size shape and structure of the nanocrystals.

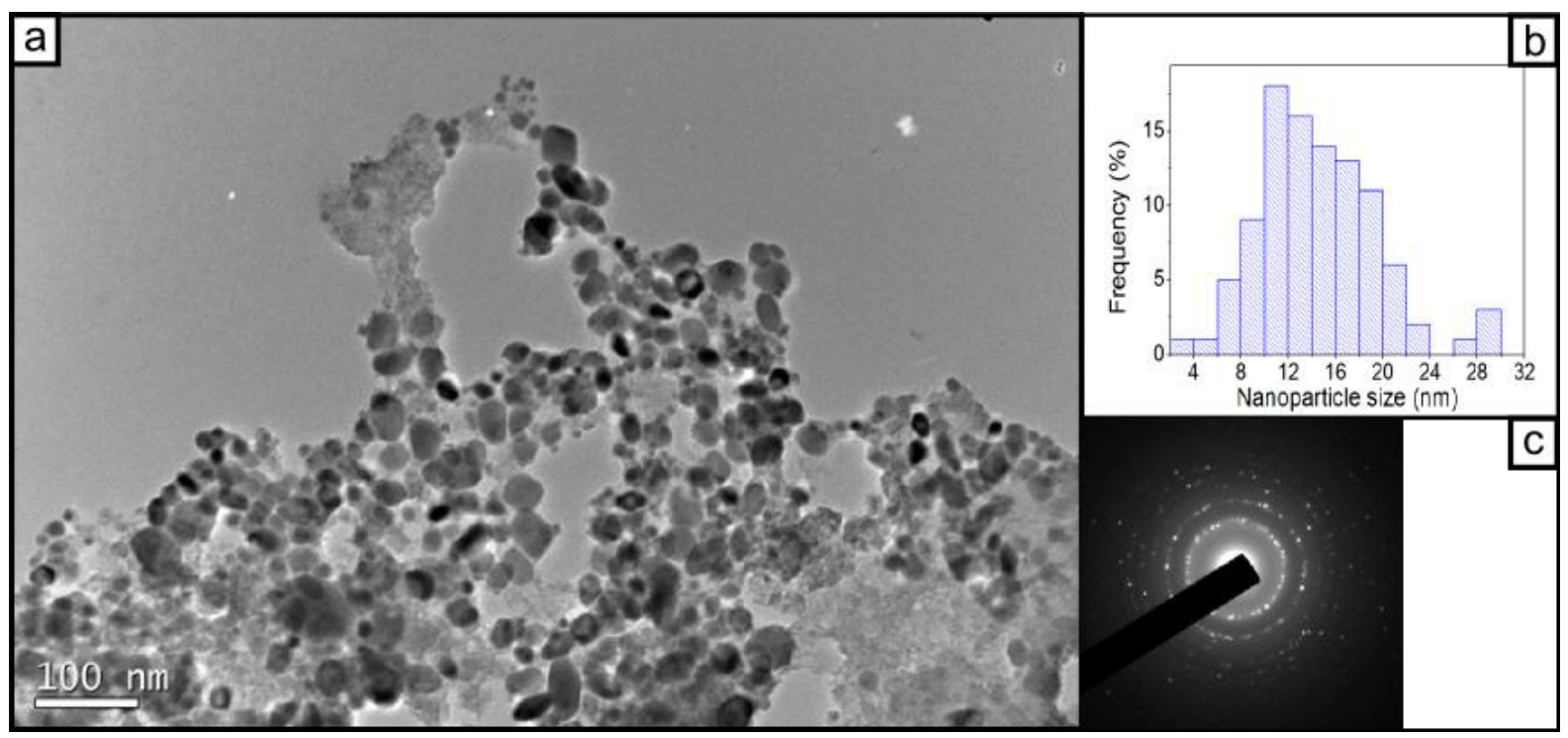

Figure 4.25: Low resolution TEM obtained in experiment 14

The nanoparticles are mostly spherical in shape. The average size is estimated at $14.5 \pm$ 13.0 from different areas of the TEM grid. The average size is larger than expected from previous reports of nanocrystals grown by microemulsion synthesis using $\mathrm{LiBH}_{4}$ (refer to chapter 3). ${ }^{42}$ The SAED diffraction rings obtained for the crystals in figure 5.5 a) do not match the CZTS structure. It can be matched to the metallic tin structure, attesting that this particular area of the TEM grid contains mostly tin nanoparticles.

The EDS analysis on six different areas of the TEM grid will permit to evaluate the ratio of copper, zinc, tin and sulphur towards the conclusion of a the synthesis of CZTS nanocrystals with a minority of other crystal impurities. Figure 4.26 represents the EDS 
spectrum obtained from the TEM image in figure 4.25 a) and table 4.14 summarizes the molar percentage of each elements on the six different areas of the TEM grid tested.

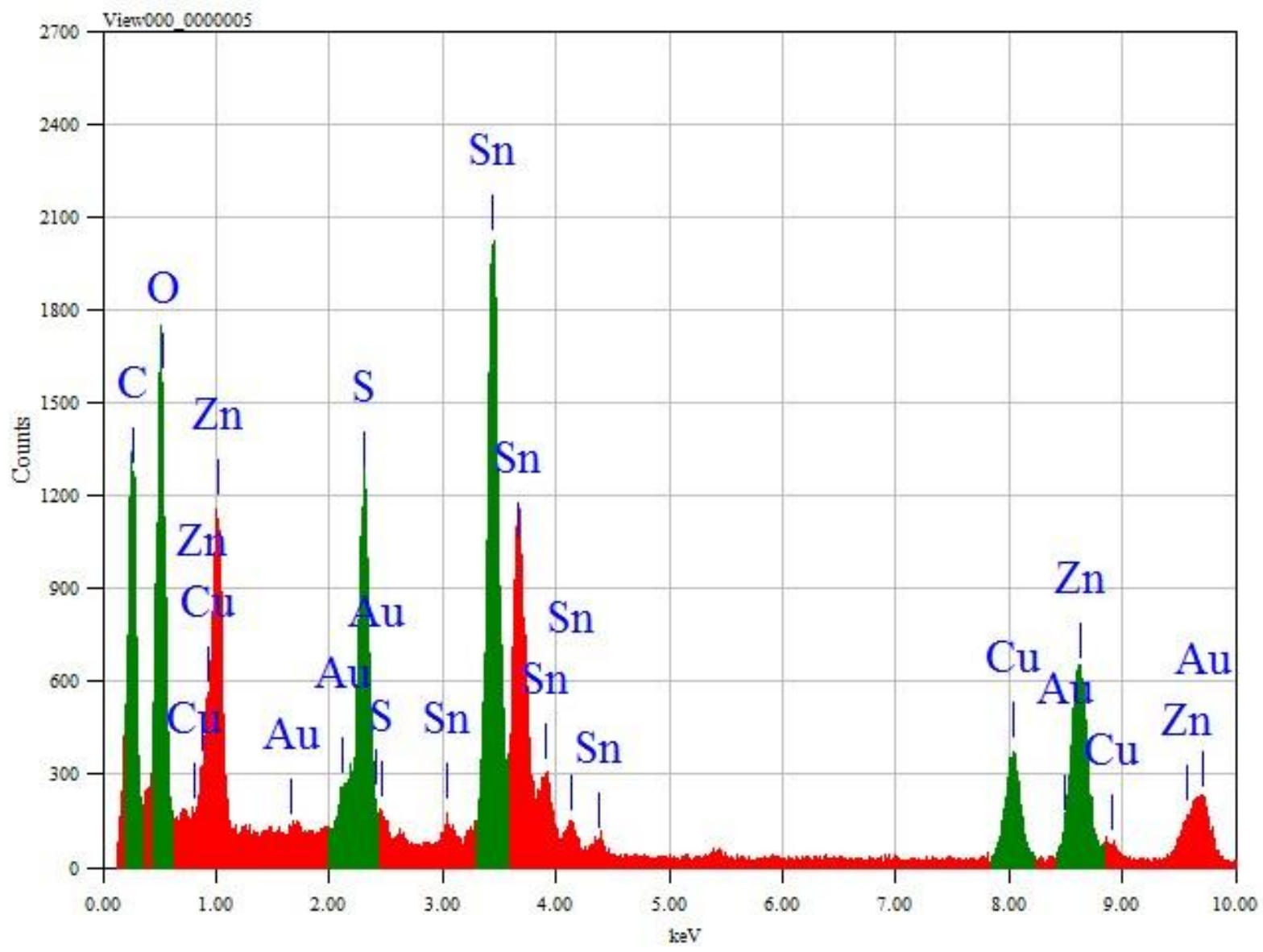

Figure 4.26: EDS pattern obtained for the area of nanocrystals shown in figure 5.25

\begin{tabular}{|c|c|c|c|c|c|c|}
\hline $\begin{array}{c}\text { EDS } \\
\text { zone }\end{array}$ & $\mathbf{C u}$ & $\mathbf{Z n}$ & $\mathbf{S n}$ & $\mathbf{S}$ & $\mathbf{C u}:(\mathbf{Z n}+\mathbf{S n})$ & $\mathbf{Z n}: \mathbf{S n}$ \\
\hline 1 & 0.8 & 1.04 & 3.44 & 1.57 & 0.18 & 0.30 \\
\hline 2 & 1.97 & 4.23 & 12.69 & 5.14 & 0.12 & 0.33 \\
\hline 3 & 1.96 & 4.48 & 6.41 & 6.02 & 0.18 & 0.70 \\
\hline 4 & 1.32 & 10.02 & 5.09 & 10.96 & 0.09 & 1.97 \\
\hline 5 & 2.73 & 5.27 & 12.31 & 6.6 & 0.16 & 0.43 \\
\hline 6 & 2.48 & 3.91 & 9.57 & 5.12 & 0.18 & 0.41 \\
\hline
\end{tabular}

Table 4.14: molar percentages of each elements and ratios calculated from EDS spectra for 4 different regions of nanocrystals obtained from experiment 14

The EDS ratios of the copper, tin, zinc and sulphur reported in table 4.13 do not correspond to the expected percentages in a $\mathrm{Cu}_{2} \mathrm{ZnSnS}_{4}$ structure. It also does not match the initial ratio of precursors used in the synthesis process. Zone 1 corresponds to the 
figure 4.25 TEM image, and the high percentage of tin compared to the other elements confirms the main presence of tin nanoparticles on that specific area. It can be concluded that the chemical reduction of the different precursors with lithium borohydride did not occur simultaneously, which resulted in the formation of several phases.

The attempts of synthesis of CZTS nanocrystals were proven difficult and the discrepancy in chemical reduction time led to the formation of multiple crystal structures.

In the next section is reported the experiments conducted towards the synthesis of each individual tin, zinc and copper sulphides to prove that microemulsion technique can produce binary nanocrystals, even though the formation of quaternary nanoparticles remains challenging.

\subsection{Microemulsion synthesis of metal sulphides}

As the chemical reduction synthesis of CZTS did not succeed in the production of pure kesterite CZTS structure, the possibility of producing each sulphide using hydride reducing agents is evaluated towards establishing whether the difference in reduction time causes the different phases. Three different experiments were conducted with the aim at the synthesis of copper sulphide, tin sulphide and copper disulphide.

\section{Experimental}

\begin{tabular}{|c|c|c|c|c|c|c|c|c|}
\hline $\begin{array}{c}\text { Exp } \\
\text { no }\end{array}$ & $\mathbf{C u}$ & Zn & Sn & S & Temperature & Solvent & Surfactant & $\begin{array}{c}\text { Reducing } \\
\text { agent }\end{array}$ \\
\hline 15 & $\begin{array}{c}2 \\
\mathrm{mmol}\end{array}$ & - & - & $\begin{array}{c}1 \\
\mathrm{mmol}\end{array}$ & $\begin{array}{c}\text { Room } \\
\text { temperature }\end{array}$ & Toluene & TOAB & $\mathrm{LiBH}_{4}$ \\
\hline 16 & - & $\begin{array}{c}1 \\
\mathrm{mmol}\end{array}$ & - & $\begin{array}{c}1 \\
\mathrm{mmol}\end{array}$ & $\begin{array}{c}\text { Room } \\
\text { temperature }\end{array}$ & Toluene & $\mathrm{TOAB}$ & $\mathrm{LiBH}_{4}$ \\
\hline 17 & - & - & $\begin{array}{c}1 \\
\mathrm{mmol}\end{array}$ & $\begin{array}{c}1 \\
\mathrm{mmol}\end{array}$ & $\begin{array}{c}\text { Room } \\
\text { temperature }\end{array}$ & Toluene & TOAB & $\mathrm{LiBH}_{4}$ \\
\hline
\end{tabular}

Table 4.15: Key variables in experiments 15, 16 and 17 
TOAB $(0.5 \mathrm{~g})$ was placed in a Schlenk tube along with either $0.41 \mathrm{~g}$ of copper acetate dihydrate or $0.22 \mathrm{~g}$ of zinc acetate or $0.23 \mathrm{~g}$ of tin dichloride, degassed under vacuum for 15 minutes then purged with nitrogen for 20 minutes. Then, $30 \mathrm{~mL}$ of anhydrous toluene was injected into the tube. After 1 hour minutes of stirring to dissolve the precursor salts, $4 \mathrm{~mL}$ of lithium borohydride solution in THF was injected in the reaction solution. After 10 minutes, $0.032 \mathrm{~g}$ of sulphur dissolved into $2 \mathrm{~mL}$ of toluene were added to the mixture. The nanoparticles were collected by centrifugation at $140.000 \mathrm{rpm}$ for 5 minutes and purified by three washings in a 1/1 mixture of methanol and toluene.

\section{Results}

The crystals structures of the obtained crystals are evaluated by XRD. The spectra represented in figure 4.27 are compared to the JCPDS standards for ZnS (card 89-2174), $\mathrm{Cu}_{2} \mathrm{~S}$ (card 26-116) and SnS (card 53-0526).

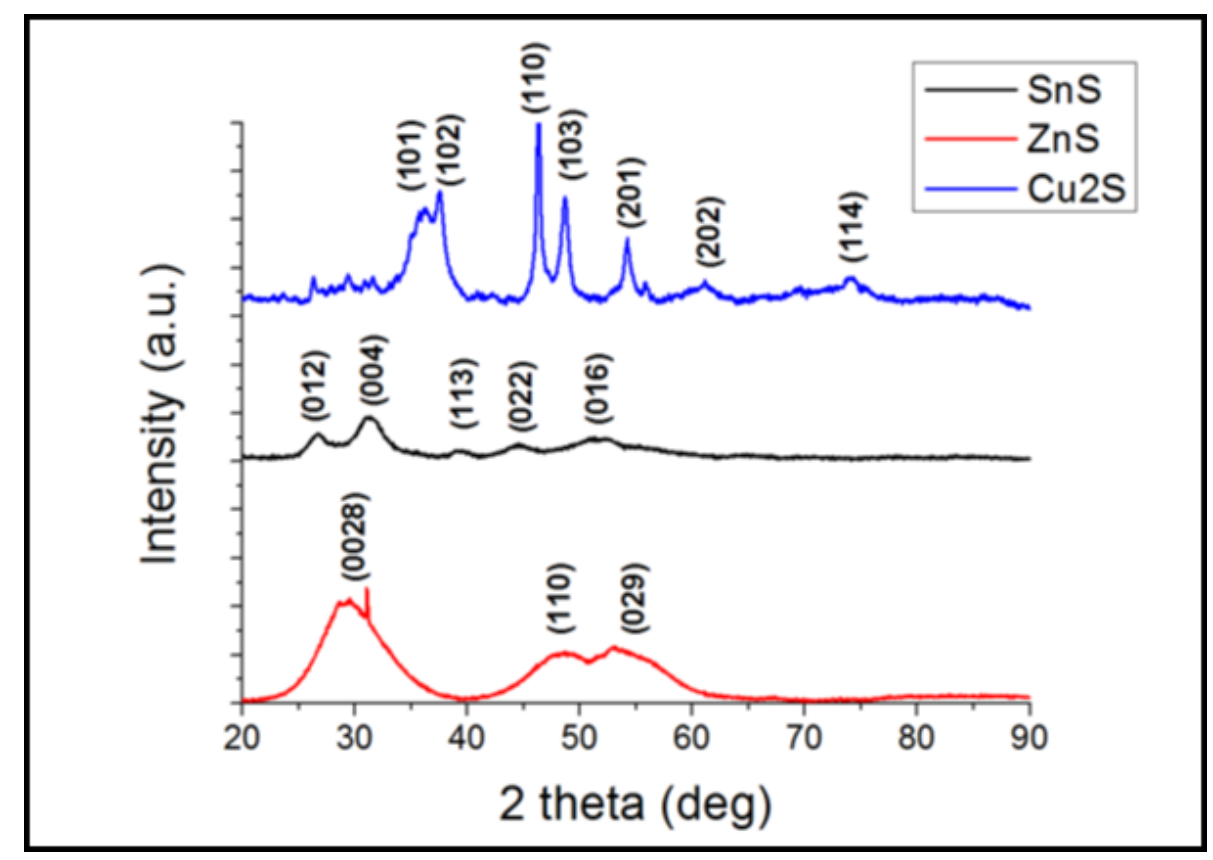

Figure 4.27: XRD spectra of $\mathrm{ZnS}, \mathrm{Cu}_{2} \mathrm{~S}$ and $\mathrm{SnS}$ 
The XRD spectrum of ZnS (red in figure 5.27) presents very broad peaks centered on the positions of the (0028), (110) and (029) reported in the JCPDS card. Those three reflections are reported to be the highest reflection intensities. It is concluded that the ZnS crystal structure (wurtzite) was synthesized for the nanoparticles in experiment 16 (black in figure 4.27). The SnS nanocrystals spectrum can be matched to the SnS standard through all the major reflections, (012), (004), (113), (022) and (016). Because no other phase was observed, the particles prepared are of pure SnS nature. Finally, the $\mathrm{Cu}_{2} \mathrm{~S}$ spectra (blue in figure 4.27) is matched to the chalcocite standard of $\mathrm{Cu}_{2} \mathrm{~S}$ with a match on all major reflections: (101), (102), (110), (103), (201), (202) and (114). 


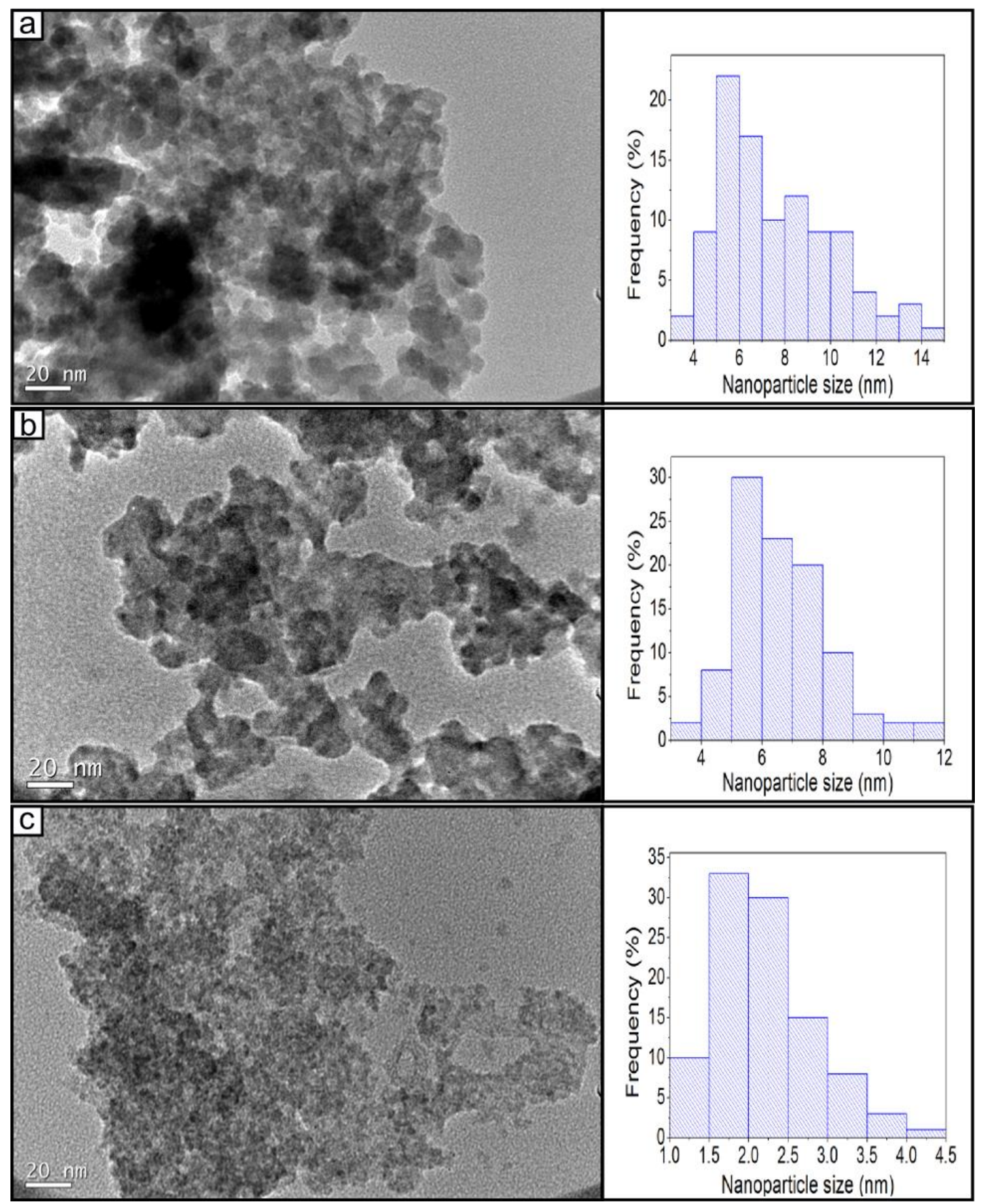

Figure 4.28: TEM images and corresponding size ditribution of a) $\mathrm{Cu}_{2} \mathrm{~S}$, b) $\mathrm{SnS}$ and c) $\mathrm{ZnS}$

TEM images in figure 4.28 show some agglomeration of the nanocrystals. It can still be distinguished the sherical shape of the nanocrystals. $\mathrm{Cu}_{2} \mathrm{~S}$ crystal sizes are in the range of $7.6 \pm 5.1 \mathrm{~nm}$, SnS nanocrystals have a size distribution of $6.6 \pm 4.1 \mathrm{~nm}$ and $\mathrm{ZnS}$ nanoparticles have an average size of $2.2 \pm 1.5 \mathrm{~nm}$. The discrepancy in sizes for the three metal sulphides is evidence for a difference in nucleation time between the 3 samples. 
The smallest average size of ZnS nanocrystals manifest of a "burst" nucleation process created by the reduction of zinc acetate by lithium borohydride.

The microemulsion synthesis was successful in the production of $\mathrm{SnS}, \mathrm{ZnS}$ and $\mathrm{Cu}_{2} \mathrm{~S}$ nanocrystals. Binary nanoparticles can be synthesized using the microemulsion technique. It is a promising stepping stone towards ternary and quaternary nanocrystals that have never been reported using the chemical reduction in reverse micelle principle.

\subsection{Discussion}

Samples taken at various temperatures between room temperature and $280^{\circ} \mathrm{C}$ reveal that pure CZTS nanocrystals of the kesterite structure were obtained after treatment at $260^{\circ} \mathrm{C}$ or higher. At lower temperatures, the CZTS particles presented an additional nanoparticle structure that can be matched to the wurtzite structure. The pure kesterite structure of $\mathrm{Cu}_{2} \mathrm{ZnSnS}_{4}$ was achieved via a solution process by Zou et al using a single complex precursor decomposing at $220^{\circ} \mathrm{C} .{ }^{43}$ Catley et al reported the wurtzite/kesterite transformation with temperature. They achieved the CZTS structure by decomposing four metal complexes as precursors. ${ }^{44}$

By comparing the CZTS nanocrystals synthesized in solvents with different coordination strength, the role of the solvent on the particle size was established. Using only oleylamine, the particles have a much smaller size than using octadecene. Oleylamine acts as a surfactant, thus limiting the size of the crystals. The binding strength of amines limits the size of nanoparticles. ${ }^{45,46}$

The synthesis via hot injection of precursors has been reported to improve the size and shape control on nanoparticle formation. ${ }^{47,48}$ The sizes of the CZTS nanocrystals synthesized by hot injection of the four precursors (exp 5) and the CZTS nanoparticles obtained by decomposing the precursors present in solution before heating (exp 2) decreases from 19.0 to $6.8 \mathrm{~nm}$ on average. Nevertheless, the simultaneous injection of the four precursors is a difficult procedure. The hot injection of tin, zinc and sulphur 
precursors in the hot solvent containing the copper precursor did not lead to the formation of pure CZTS, because of a difference in decomposition times of the precursors.

Chemical reduction synthesis is expected to produce smaller particles than high temperature decomposition. ${ }^{49}$ It was first proposed to study the effect of using a reducing agent combined to the high temperature decomposition. As injecting a hydride reducing agent into a hot solvent would create a very violent and dangerous reaction, the four precursors with $\mathrm{LiAlH}_{4}$ or $\mathrm{LiBH}_{4}$ were reduced before the heating treatment. This technique led to the creation of multiple phases, not a pure kesterite phase.

As combining both chemical reduction and high temperature decomposition was still unsuccessful in producing small monodisperse CZTS nanocrystals, a new approach in the synthesis of CZTS by reduction in microemulsion was investigated. Using microemulsions to synthesize nanoparticles is expected to reduce the particle size compared to the high temperature method due to the micelle confinement of the metal precursors. ${ }^{50}$ Other reported synthesis involving this technique involves mostly single metal oxides or metallic particles. ${ }^{51,52}$ Microemulsion synthesis was previously reported by the Tilley group to produce Si and Ge quantum dots. ${ }^{32,33}$ No CZTS nanocrystal synthesis have been published in the literature using the microemulsion method. Additionally, this synthetic process does not require high temperature and is conducted at room temperature. Only $\mathrm{Cu}_{2} \mathrm{ZnSnS}_{4}$ thin films have been synthesized at room temperature by Shinde et al using a successive ionic layer adsorption and reaction (SILAR) method. ${ }^{53}$ Our attempts at chemical reduction in reverse-micelle environment to form CZTS was not successful in the production of the CZTS phase, but generated particles of various phases with a significant decrease of particle size down to around $4 \mathrm{~nm}$ (exp 13). Thhis can be explained in terms of the reduction ability of the four precursors by the hydride reducing agent differ. This leads to a disparity of nucleation events of the four elements copper, tin and zinc, that then react a different time with the elemental sulphur, resulting in the synthesis of nanocrystals with various composition and crystal phases.

Finally, the chemical reduction in reverse micelle synthesis principles was applied to synthesize each metal sulphide individually: $\mathrm{Cu}_{2} \mathrm{~S}, \mathrm{SnS}$ and $\mathrm{ZnS}$. XRD analysis confirmed 
the pure crystal phases of each of the three metal sulphides. TEM confirms the production of nanoparticles with a narrow size distribution. ZnS presented the smallest size, down to around $2.2 \mathrm{~nm}$. The size of our nanocrystals is smaller than the ones reported by $\mathrm{Xu}$ et al via hydrothermal solution synthesis. ${ }^{54}$ Using a high temperature synthesis, Ning et al produced monodisperse nanoparticles with an average size of 4.6 $\mathrm{nm} .{ }^{55}$ In the Tilley group, Dr. Sherry Xu et al produced $3 \mathrm{~nm}$ size water soluble SnS nanoparticle using the high temperature method. ${ }^{56}$ Our method reported here succeeded in the production of SnS particles with a $6.6 \mathrm{~nm}$ average size. In comparison, $\mathrm{Cu}_{2} \mathrm{~S}$ has been more reported than the two other single metal sulphides reported in this study. It developed a large interest due to its semiconducting properties and possible applications in solar cells. ${ }^{57}$ Korgel was the pioneer in the synthesis of $\mathrm{Cu}_{2} \mathrm{~S}$ nanostructures in 2003 with a solventless method. ${ }^{58}$ The Li group reported highly monodisperse $\mathrm{Cu}_{2} \mathrm{~S}$ nanocrystals with an average size of $8.2 \mathrm{~nm}$ using an autoclave synthesis. ${ }^{59}$ The shape of the $\mathrm{Cu}_{2} \mathrm{~S}$ nanocrystals obtained in this study are not up to the shape control standard of $\mathrm{Li}$ and Korgel, but the $7.6 \mathrm{~nm}$ average size we observe is a great step towards future work on shape control and/or device application in solar cells.

The changes in size vs. crystal structure for the metal sulphides supports the hypothesis that the difference in reducing abilities of the precursors causes multiple phases when using the reverse micelle process. For future work, it is possible that different precursors or complexes of precursor containing all the desired elements would be the best approach to overcome this problem. 


\subsection{References}

1_Yang, Y.; Zhou, H.-P.; Hsu, W.-C.; Duan, H.-S.; Bob, B.; Yang, W.; Song, T.-b.; Hsu, C.-J., CZTS Nanocrystals: A promising approach for next generation thin film photovoltaics. Energy \& Environmental Science 2013.

2_Wang, J.; Xin, X.; Lin, Z., Cu2ZnSnS4 nanocrystals and graphene quantum dots for photovoltaics. Nanoscale 2011, 3 (8), 3040-3048.

3_Panthani, M. G.; Akhavan, V.; Goodfellow, B.; Schmidtke, J. P.; Dunn, L.; Dodabalapur, A.; Barbara, P. F.; Korgel, B. A., Synthesis of CulnS2, CulnSe2, and Cu(InxGa1-x)Se2 (CIGS) Nanocrystal "Inks" for Printable Photovoltaics. Journal of the American Chemical Society 2008, 130 (49), 16770-16777.

4_http://www.nanocotechnologies.com/content/AdvancedMaterials/CIGSCISNanoparti cle.asp

5_Mitzi, D. B.; Gunawan, O.; Todorov, T. K.; Wang, K.; Guha, S., The path towards a high-performance solution-processed kesterite solar cell. Solar Energy Materials and Solar Cells 2011, 95 (6), 1421-1436.

6_ Wang, K.; Shin, B.; Reuter, K. B.; Todorov, T.; Mitzi, D. B.; Guha, S., Structural and elemental characterization of high efficiency $\mathrm{Cu}_{2} \mathrm{ZnSnS}_{4}$ solar cells. Applied Physics Letters 2011, 98 (5), 051912-3.

7_ Todorov, T. K.; Reuter, K. B.; Mitzi, D. B., High-Efficiency Solar Cell with EarthAbundant Liquid-Processed Absorber. advanced materials 2010, 22 (20), E156-E159.

8_ Steinhagen, C.; Panthani, M. G.; Akhavan, V.; Goodfellow, B.; Koo, B.; Korgel, B. A., Synthesis of Cu2ZnSnS4 Nanocrystals for Use in Low-Cost Photovoltaics. Journal of the American Chemical Society 2009, 131 (35), 12554-12555. 
9_ Riha, S. C.; Parkinson, B. A.; Prieto, A. L., Solution-Based Synthesis and Characterization of Cu2ZnSnS4 Nanocrystals. Journal of the American Chemical Society 2009, 131 (34), 12054-12055.

10_Khare, A.; Wills, A. W.; Ammerman, L. M.; Norris, D. J.; Aydil, E. S., Size control and quantum confinement in Cu2ZnSnS4 nanocrystals. Chemical Communications 2011, 47 (42), 11721-11723.

11_Oliveira, J. F. A.; Milão, T. M.; Araújo, V. D.; Moreira, M. L.; Longo, E.; Bernardi, M. I. B., Influence of different solvents on the structural, optical and morphological properties of CdS nanoparticles. Journal of Alloys and Compounds 2011, 509 (24), 6880-6883.

12_Ranjan, B.; Kailasnath, M.; Kumar, N.; Radhakrishnan, P.; Achari, S.; Nampoori, V. P. N., Influence of Solvent on Size and Properties of ZnO Nanoparticles. AIP Conference Proceedings 2009, 1147 (1), 287-296.

13_Watt, J.; Cheong, S.; Tilley, R. D., How to control the shape of metal nanostructures in organic solution phase synthesis for plasmonics and catalysis. Nano Today 2013, 8 (2), 198-215.

14_Dang, T. M. D.; Le, T. T. T.; Fribourg-Blanc, E.; Dang, M. C., The influence of solvents and surfactants on the preparation of copper nanoparticles by a chemical reduction method. Advances in Natural Sciences: Nanoscience and Nanotechnology 2011, 2 (2), 025004.

15_Tao, A. R.; Habas, S.; Yang, P., Shape Control of Colloidal Metal Nanocrystals. Small 2008, 4 (3), 310-325.

16_Biacchi, A. J.; Schaak, R. E., The Solvent Matters: Kinetic versus Thermodynamic Shape Control in the Polyol Synthesis of Rhodium Nanoparticles. ACS Nano 2011, 5 (10), 8089-8099.

17_Lu, X.; Zhuang, Z.; Peng, Q.; Li, Y., Wurtzite Cu2ZnSnS4 nanocrystals: a novel quaternary semiconductor. Chemical Communications 2011, 47 (11), 3141-3143. 
18_ Hinz, W., Nucleation and crystal growth. Journal of Non-Crystalline Solids 1977, 25 (1-3), 215-260.

19_Heyer, H., The Kinetics of Crystal Growth. Angewandte Chemie International Edition in English 1966, 5 (1), 67-77.

20_Guo, Q.; Hillhouse, H. W.; Agrawal, R., Synthesis of Cu2ZnSnS4 Nanocrystal Ink and Its Use for Solar Cells. Journal of the American Chemical Society 2009, 131 (33), 1167211673.

21_Ito, K.; Nakazawa, T., Electrical and Optical Properties of Stannite-Type Quaternary Semiconductor Thin Films. Japanese Journal of Applied Physics 1988, 27 (Part 1, No), 2094-2097.

22_ Katagiri, H., Cu2ZnSnS4 thin film solar cells. Thin Solid Films 2005, 480-481 (0), 426432.

23_Piper, W. W.; Marple, D. T. F.; Johnson, P. D., Optical Properties of Hexagonal ZnS Single Crystals. Physical Review 1958, 110 (2), 323-326.

24_Crouse, C. A.; Barron, A. R., Reagent control over the size, uniformity, and composition of Co-Fe-O nanoparticles. Journal of Materials Chemistry 2008, 18 (35), 4146-4153.

24_ Langford, J.I.; Wilson A.J.C., "Scherrer after Sixty Years: A Survey and Some New Results in the Determination of Crystallite Size," Journal of Applied Crystallography 1978, 11, pp 102-113.

25_LaMer, V. K.; Dinegar, R. H., Theory, Production and Mechanism of Formation of Monodispersed Hydrosols. Journal of the American Chemical Society 1950, 72 (11), 4847-4854. 
26_ Tanusevski, A., Optical and photoelectric properties of SnS thin films prepared by chemical bath deposition. Semiconductor Science and Technology 2003, 18 (6), 501.

27_Shiohara, A.; Prabakar, S.; Faramus, A.; Hsu, C.-Y.; Lai, P.-S.; Northcote, P. T.; Tilley, R. D., Sized controlled synthesis, purification, and cell studies with silicon quantum dots. Nanoscale 2011, 3 (8), 3364-3370.

28_Hu, J. Q.; Bando, Y.; Zhan, J. H.; Golberg, D., Fabrication of ZnS/SiC nanocables, SiCshelled ZnS nanoribbons (and sheets), and SiC nanotubes (and tubes). Applied Physics Letters 2004, 85 (14), 2932-2934.

29_Meldrum, A.; Zuhr, R. A.; Sonder, E.; Budai, J. D.; White, C. W.; Boatner, L. A.; Ewing, R. C.; Henderson, D. O., Formation of oriented particles in an amorphous host: ZnS nanocrystals in silicon. Applied Physics Letters 1999, 74 (5), 697-699.

30_Fu, X.; Yang, X.; Qiu, Z.; Zhao, F.; Zhuang, J.; He, A.; Chen, L.; Wu, C.; Duan, X.; Liang, C.; $\mathrm{Wu}, \mathrm{M}$., Outward conversion of core-shell nanostructured ZnS microspheres to mesoporous ZnO ones. CrystEngComm 2013, 15 (17), 3334-3340.

31_Zarur, A. J.; Ying, J. Y., Reverse microemulsion synthesis of nanostructured complex oxides for catalytic combustion. Nature 2000, 403 (6765), 65-67.

32_ Tilley, R. D.; Yamamoto, K., The Microemulsion Synthesis of Hydrophobic and Hydrophilic Silicon Nanocrystals. advanced materials 2006, 18 (15), 2053-2056.

33_Prabakar, S.; Shiohara, A.; Hanada, S.; Fujioka, K.; Yamamoto, K.; Tilley, R. D., Size Controlled Synthesis of Germanium Nanocrystals by Hydride Reducing Agents and Their Biological Applications. Chemistry of Materials 2009, 22 (2), 482-486. 
34_Shi, L.; Pei, C.; Xu, Y.; Li, Q., Template-Directed Synthesis of Ordered SingleCrystalline Nanowires Arrays of Cu2ZnSnS4 and Cu2ZnSnSe4. Journal of the American Chemical Society 2011, 133 (27), 10328-10331.

35_Su, Z.; Yan, C.; Tang, D.; Sun, K.; Han, Z.; Liu, F.; Lai, Y.; Li, J.; Liu, Y., Fabrication of Cu2ZnSnS4 nanowires and nanotubes based on AAO templates. CrystEngComm 2012, 14 (3), 782-785.

36_Zhao, Q.; Xu, X.; Zhang, H.; Chen, Y.; Xu, J.; Yu, D., Catalyst-free growth of singlecrystalline alumina nanowire arrays. Applied Physics A 2004, 79 (7), 1721-1724.

37_Zhou, J.; Deng, S. Z.; Chen, J.; She, J. C.; Xu, N. S., Synthesis of crystalline alumina nanowires and nanotrees. Chemical Physics Letters 2002, 365 (5-6), 505-508.

38_Akhavan, V. A.; Goodfellow, B. W.; Panthani, M. G.; Steinhagen, C.; Harvey, T. B.; Stolle, C. J.; Korgel, B. A., Colloidal CIGS and CZTS nanocrystals: A precursor route to printed photovoltaics. Journal of Solid State Chemistry 2012, 189 (0), 2-12.

39_Zaberca, O.; Gillorin, A.; Durand, B.; Chane-Ching, J. Y., A general route to the synthesis of surfactant-free, solvent-dispersible ternary and quaternary chalcogenide nanocrystals. Journal of Materials Chemistry 2011, 21 (18), 6483-6486.

40_ Yang, H.; Jauregui, L. A.; Zhang, G.; Chen, Y. P.; Wu, Y., Nontoxic and Abundant Copper Zinc Tin Sulfide Nanocrystals for Potential High-Temperature Thermoelectric Energy Harvesting. Nano Letters 2012, 12 (2), 540-545.

41_Riha, S. C.; Parkinson, B. A.; Prieto, A. L., Compositionally Tunable $\mathrm{Cu}_{2} \mathrm{ZnSn}\left(\mathrm{S}_{1-x} \mathrm{Se}_{x}\right)_{4}$ Nanocrystals: Probing the Effect of Se-Inclusion in Mixed Chalcogenide Thin Films. Journal of the American Chemical Society 2011, 133 (39), 15272-15275.

42_Shiohara, A.; Prabakar, S.; Faramus, A.; Hsu, C.-Y.; Lai, P.-S.; Northcote, P. T.; Tilley, R. D., Sized controlled synthesis, purification, and cell studies with silicon quantum dots. Nanoscale 2011, 3 (8), 3364-3370. 
43_Zou, C.; Zhang, L.; Lin, D.; Yang, Y.; Li, Q.; Xu, X.; Chen, X. a.; Huang, S., Facile synthesis of Cu2ZnSnS4 nanocrystals. CrystEngComm 2011, 13 (10), 3310-3313.

44_Cattley, C. A.; Cheng, C.; Fairclough, S. M.; Droessler, L. M.; Young, N. P.; Warner, J. H.; Smith, J. M.; Assender, H. E.; Watt, A. A. R., Low temperature phase selective synthesis of $\mathrm{Cu}_{2} \mathrm{ZnSnS}_{4}$ quantum dots. Chemical Communications 2013, 49 (36), 37453747.

45_ Walker, F. A., Steric and electronic effeccts in the coordination of amines to a cobalt(II) porphyrin. Journal of the American Chemical Society 1973, 95 (4), 1150-1153.

46_Sudhakar Reddy, J.; Sayari, A., Nanoporous zirconium oxide prepared using the supramolecular templating approach. Catalysis Letters 1996, 38 (3-4), 219-223.

47_de Mello Donegá, C.; Liljeroth, P.; Vanmaekelbergh, D., Physicochemical Evaluation of the Hot-Injection Method, a Synthesis Route for Monodisperse Nanocrystals. Small 2005, 1 (12), 1152-1162.

48_Puntes, V. F.; Krishnan, K. M.; Alivisatos, A. P., Colloidal Nanocrystal Shape and Size Control: The Case of Cobalt. Science 2001, 291 (5511), 2115-2117.

49_Sakai, T.; Alexandridis, P., Mechanism of Gold Metal Ion Reduction, Nanoparticle Growth and Size Control in Aqueous Amphiphilic Block Copolymer Solutions at Ambient Conditions. The Journal of Physical Chemistry B 2005, 109 (16), 7766-7777.

50_ Li, Y.; Park, C. W., Particle Size Distribution in the Synthesis of Nanoparticles Using Microemulsions. Langmuir 1998, 15 (4), 952-956.

51_ Zhang, X.; Chan, K.-Y., Water-in-Oil Microemulsion Synthesis of Platinum-Ruthenium Nanoparticles, Their Characterization and Electrocatalytic Properties. Chemistry of Materials 2002, 15 (2), 451-459. 
52_Hingorani, S.; Pillai, V.; Kumar, P.; Multani, M. S.; Shah, D. O., Microemulsion mediated synthesis of zinc-oxide nanoparticles for varistor studies. Materials Research Bulletin 1993, 28 (12), 1303-1310.

53_Shinde, N. M.; Dubal, D. P.; Dhawale, D. S.; Lokhande, C. D.; Kim, J. H.; Moon, J. H., Room temperature novel chemical synthesis of $\mathrm{Cu}_{2} \mathrm{ZnSnS}_{4}$ (CZTS) absorbing layer for photovoltaic application. Materials Research Bulletin 2012, 47 (2), 302-307.

54_Xu, S. J.; Chua, S. J.; Liu, B.; Gan, L. M.; Chew, C. H.; Xu, G. Q., Luminescence characteristics of impurities-activated ZnS nanocrystals prepared in microemulsion with hydrothermal treatment. Applied Physics Letters 1998, 73 (4), 478-480.

55_Ning, J.; Men, K.; Xiao, G.; Wang, L.; Dai, Q.; Zou, B.; Liu, B.; Zou, G., Facile synthesis of iv-vi SnS nanocrystals with shape and size control: Nanoparticles, nanoflowers and amorphous nanosheets. Nanoscale 2010, 2 (9), 1699-1703.

56_ Xu, Y.; Al-Salim, N.; Tilley, R. D., Synthesis and Size Dependent Reflectance Study of Water Soluble SnS Nanoparticles. Nanomaterials 2012, 2 (1), 54-64.

57_Lee, H.; Yoon, S. W.; Kim, E. J.; Park, J., In-Situ Growth of Copper Sulfide Nanocrystals on Multiwalled Carbon Nanotubes and Their Application as Novel Solar Cell and Amperometric Glucose Sensor Materials. Nano Letters 2007, 7 (3), 778-784.

58_Sigman, M. B.; Ghezelbash, A.; Hanrath, T.; Saunders, A. E.; Lee, F.; Korgel, B. A., Solventless Synthesis of Monodisperse Cu2S Nanorods, Nanodisks, and Nanoplatelets. Journal of the American Chemical Society 2003, 125 (51), 16050-16057.

59_Zhuang, Z.; Peng, Q.; Zhang, B.; Li, Y., Controllable Synthesis of Cu2S Nanocrystals and Their Assembly into a Superlattice. Journal of the American Chemical Society 2008, $130(32), 10482-1483$ 


\section{Germanium, tin and tin/germanium alloy nanoparticles}

After silicon, tin and germanium are the next most abundant elements from the group IV. All semiconductors from this material group have great properties that make them of interest for electronic applications. ${ }^{1-3}$ After studying silicon quantum dots in chapter 3 , this chapter looks into germanium quantum dots and tin nanoparticles.

Germanium quantum dots (GeQDs) have been significantly less studied than silicon quantum dots, but present similar properties. They exhibit a strong blue/green fluorescence. The larger Bohr radius allows GeQDs to reach the quantum confinement regime (and thus the photoluminescence properties) at larger particle size than in the case for silicon. ${ }^{4,5}$ There is just a few reports of the influence of the surface passivation on the optical properties of germanium nanocrystals, so in this study it is proposed to investigate the optical properties of germanium nanocrystals capped with four different surface ligands.

Tin can be found in two different solid state phases. The most common phase of tin is a metal, called $\beta$. Below $13^{\circ} \mathrm{C}$, tin transforms into what is commonly known as grey tin or tin pest, which has semiconductor properties. The semiconducting phase of tin (or $\alpha$-tin) presents the same diamond cubic structure than silicon and germanium. The bulk $\alpha$-tin has a low direct band gap $0.08 \mathrm{eV}$ and nanoparticles are expected to have a large band gap for photovoltaic applications when their dimensions are in the quantum confined region. ${ }^{6}$ Also the nanoparticle form is expected to increase the temperature stability of the $\alpha$-tin phase. ${ }^{7}$ So far, no solution phase synthesis of these particles has been reported in the literature.

Due to their similar crystal structures, tin and germanium can be alloyed and modified properties compared to their single material properties are expected, such as a direct band gap. ${ }^{8,9}$ But due to their difference in lattice parameter, tin-germanium alloys are challenging. Tin-germanium alloys have not yet been synthesized via solution phase. 
Physical methods, such as molecular beam epitaxy, have been reported to be successful in the production of tin-germanium alloy nanostructures. ${ }^{10-13}$

In this research, germanium quantum dots and tin nanoparticles were synthesized by chemical reduction using a hydride reducing agent. To achieve the semiconducting $\alpha$-tin phase, tin was alloyed with its structural germanium homologue. Nanocrystals were characterized by TEM, XRD and UV-Visible and photoluminescence spectroscopies.

\subsection{Germanium quantum dots synthesis and optical properties}

The solution phase synthesis methods are the most reported for the formation of germanium nanoparticles. These methods involve either chemical reduction or high temperature decomposition of germanium precursors. Kauzlarich et al reported a solution method using a combination of $\mathrm{NaGe}$ with $\mathrm{GeCl}_{4}$ or $\mathrm{NH}_{4} \mathrm{Br}^{13,14} \mathrm{Lu}$ and coworkers formed germanium nanocrystals by thermolysis of germanium precursors in supercritical solvents. ${ }^{15}$ An ultrasonic solution reduction method based on the reduction of $\mathrm{GeCl}_{4}$ by metal hydride or alkaline in ambient condition has been reported by Wu et al. ${ }^{16}$ The nanocrystals with an average $5 \mathrm{~nm}$ size exhibit a photoluminescence emission with a maximum at $352 \mathrm{~nm}$. Wilcoxon and co-workers described an inverse micelles synthesis by reduction of an ionic salt by lithium aluminium hydride. A PL in the range 350-700 $\mathrm{nm}$ was observed from nanocrystals $2-5 \mathrm{~nm}$ in size. ${ }^{17}$ In this section, GeQDs were synthesized using the microemulsion method developed by the Tilley group. ${ }^{18}$ This technique yields highly luminescent nanoparticles. These were then investigated in respect of the influence of four capping molecules on their optical properties. Lithium borohydride was chosen to reduce the germanium salt as it has been previously shown to produce small monodisperse silicon quantum dots (see chapter 3 ).

\section{Experimental}

In a typical experiment, $1 \mathrm{~g}$ of tetraoctylammonium bromide (TOAB) was dissolved in 50 $\mathrm{mL}$ of anhydrous toluene under nitrogen. After 10 minutes of active stirring, $0.3 \mathrm{~mL}$ of $\mathrm{GeCl}_{4}$ was injected into the solution. The solution was stirred for 1 hour to form reverse micelles. Then, $4 \mathrm{~mL}$ of $\mathrm{LiBH}_{4}$ were swiftly injected into the Schlenk tube. The solution was left to react for one hour. The solution was then transferred into a quartz tube, 
preliminary purged and filled with $\mathrm{N}_{2}$. For amine terminated GeQDs, $2 \mathrm{~mL}$ of allylamine were injected into the solution. For dodecene surface capping, $2 \mathrm{~mL}$ of dodecene were injected in the quartz tube. The solution was then exposed to UV-light at $254 \mathrm{~nm}$ for four hours.

Amine terminated GeQDs were purified by size exclusion column chromatography. The solution after UV treatment was filtered using a Fisher Scientific paper filter with a 0.5 $\mu m$ pore size, and then concentrated to $2 \mathrm{~mL}$ with a rotary evaporator. After five minutes of homogenization in an ultrasonic bath, the solution was then put on top of the column $(\phi=1 \mathrm{~cm}, 41.0 \mathrm{~cm})$, containing Sephadex gel LH-20 (beads size 25-100 $\mu \mathrm{m}$ ) as the stationary phase in HPLC grade methanol. The flow rate was set to one drop every four seconds, and fractions were collected every 50 drops. Each fraction was checked for luminescence with a handheld UV lamp (365 nm). Luminescent fractions were collected and concentrated to $1 \mathrm{~mL}$ under vacuum. The size exclusion process was repeated three times to obtain pure surface passivated germanium quantum dots.

Dodecene terminated GeQDs were purified by liquid/liquid phase separation. The UV treated solution was filtered using the $0.5 \mu \mathrm{m}$ pore size filter paper then the toluene was evaporated using a rotary evaporator. The nanoparticles were dissolved in $10 \mathrm{~mL}$ of hexane and then placed in a separating funnel along with $10 \mathrm{~mL}$ of $\mathrm{N}$-methylformamide. After 3 washes with $\mathrm{N}$-methylformamide, 3 more washes were done using deionised water.

Epoxy terminated GeQDs were obtained by reacting dodecene terminated GeQDs dissolved in $10 \mathrm{~mL}$ of dichloromethane with $0.3 \mathrm{~g}$ of meta-chloroperoxybenzoic acid under active stirring for 6 hours. The particles were washed by liquid-liquid phase separation using a saturated sodium sulphite solution as the aqueous phase for 2 washes and then two more washes using a $10 \mathrm{M}$ sodium hydroxide solution.

Diol terminated GeQDs were obtained by reacting epoxy-terminated GeQDs dissolved in $1 \mathrm{~mL}$ chloroform and $2 \mathrm{~mL}$ of water with $2 \mathrm{mg}$ of tetrabutylammonium hydrogen sulphate by stirring for $16 \mathrm{~h}$ at $40^{\circ} \mathrm{C}$. The mixture was washed with $10 \mathrm{~mL}$ of diethyl ether for 3 times. 
The surface chemistry of germanium quantum dots and the chemical reactions are presented in scheme 5.1 .

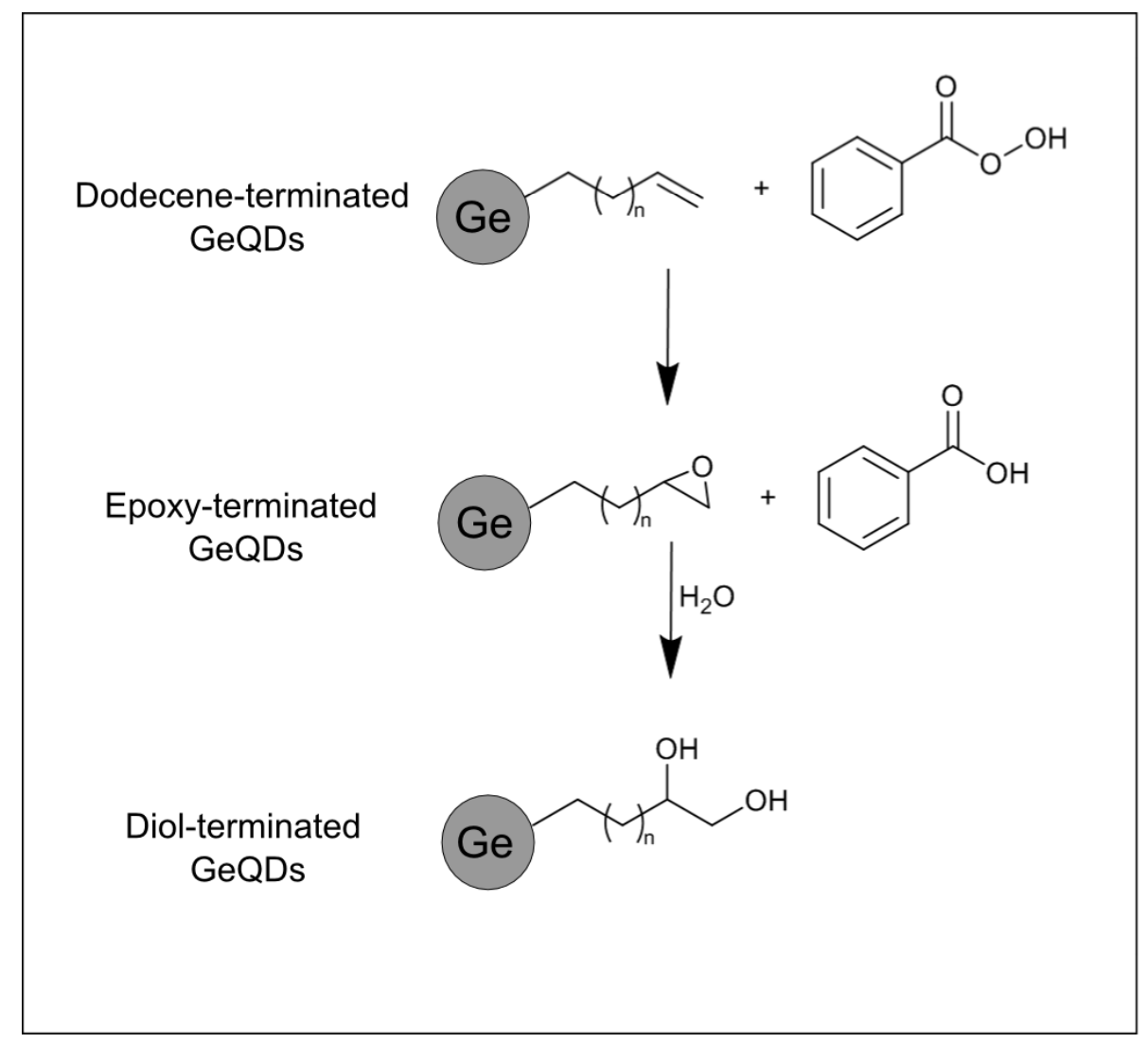

Scheme 5.1: Chemical reactions on the surface of germanium quantum dots to produce dodecene-epoxy- and diol-terminated GeQDs

\section{Characterization}

The average size and shape of the nanoparticles is determined using transmission electron microscopy. Figure 5.1 represents the TEM images obtained for germanium quantum dots with the four different surface terminations: amine, dodecyl, epoxy and diol and their corresponding size distribution diagrams. 


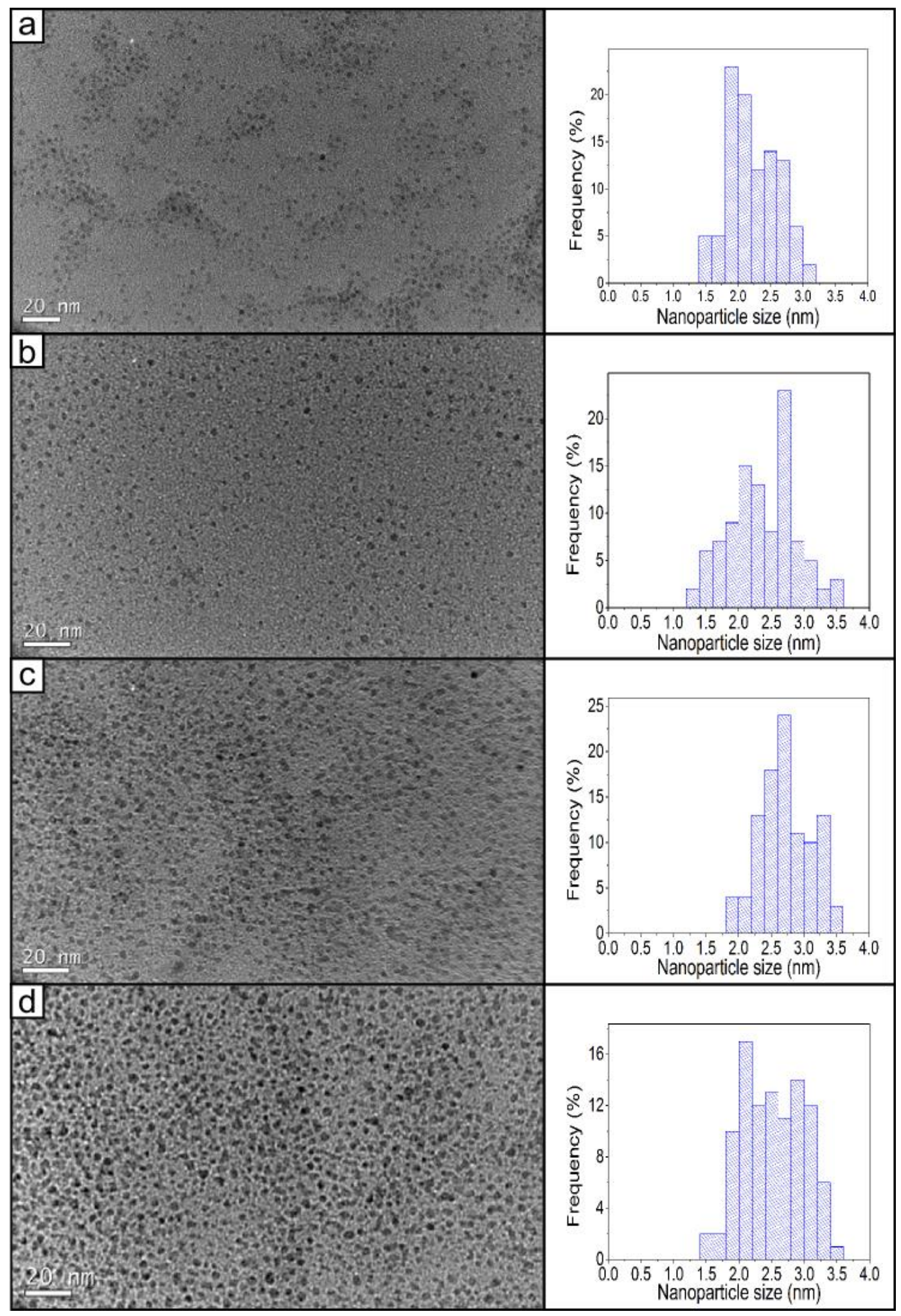

Figure 5.1: TEM images obtained for germanium nanocrystals terminated with a) allylamine, b) dodecene, c) epoxy and d) diol and their corresponding size distribution diagrams. 
The four GeQDs samples present a regular spherical shape. The average size is estimated by measuring 200 nanoparticles from different areas of the TEM grid. Amineterminated GeQDs are $2.3 \pm 0.8 \mathrm{~nm}$ on average, dodecene-terminated ones $2.4 \pm 1.2$ $\mathrm{nm}$, epoxy-terminated $2.6 \pm 0.8 \mathrm{~nm}$ and diol-terminated GeQDs average at $2.5 \pm 1.0 \mathrm{~nm}$. The slight difference in average sizes between the samples is most likely due to measuring errors or a different electron density around the quantum dots affecting the apparent size of the nanocrystals. In conclusion, the size of the four different capped germanium nanocrystals is not affected by the surface capping as it occurs post-growth process.

The absorption process of the four samples of germanium quantum dots was determined by UV-Visible spectroscopy. Amine terminated GeQDs were dispersed in water, diol-, epoxy- and dodecyl-terminated GeQDs were dispersed in chloroform. The four spectra are presented in figure 5.2 .

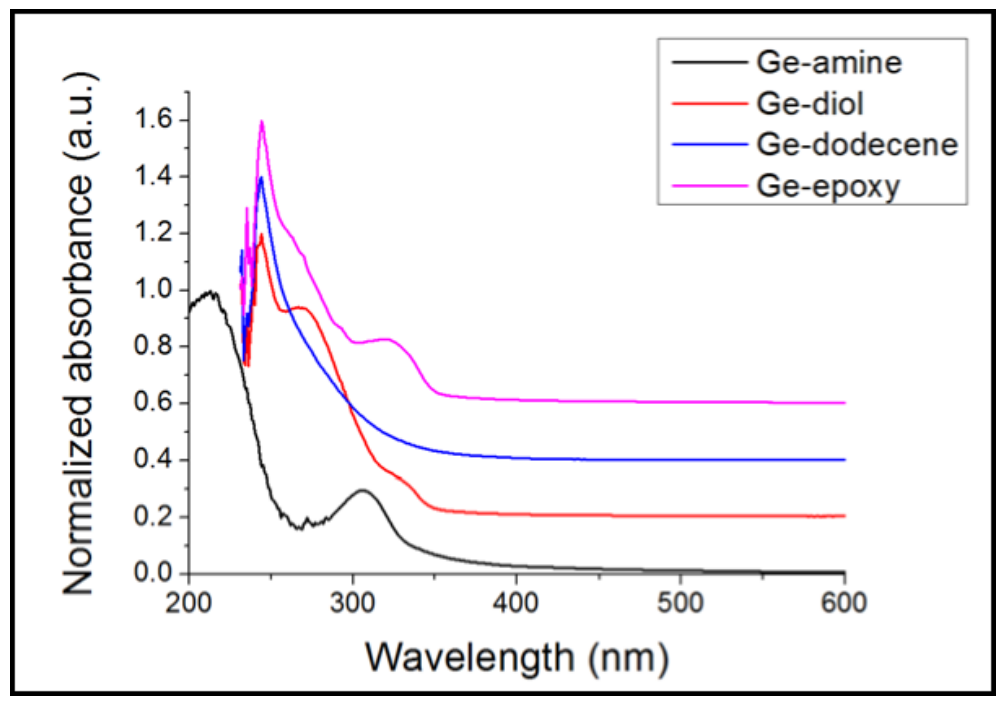

Figure 5.2: Absorbance spectra for germanium nanocrystals capped with allylamine, diol, dodecene and epoxy.

The germanium nanocrystals terminated with amine, alkyl, epoxy or diol groups in solution present absorbances in the UV region. The dodecyl-, diol- and epoxy terminated GeQDs absorb wavelengths shorter than $350 \mathrm{~nm}$ with a sharp peak at $255 \mathrm{~nm}$. Diol and epoxy surface capping molecules exhibit three local maxima at 255, 270 and $330 \mathrm{~nm}$, attesting that three absorption process are occurring in the germanium nanocrystals. 
Amine terminated GeQDs present two absorption peaks at 220 and $310 \mathrm{~nm}$ and an absorption edge around $410 \mathrm{~nm}$. This variation of absorption peak and edge wavelengths indicates an influence of the capping molecule over the optical properties. Dodecyl-, diol- and epoxy terminated GeQDs present similar peak features but at different peak intensities while the absorption spectrum of the GeQDs with allylamine ligand is significantly different. This difference can also be explained by the solvent: water for the amine terminated sample and chloroform for the three other samples.

The fluorescence of germanium nanocrystals is evaluated with photoluminescence spectroscopy. The same solutions as those used for the absorption were utilized. Spectra were recorded between 280 and $400 \mathrm{~nm}$ at excitation wavelength intervals of $20 \mathrm{~nm}$. The range of emission wavelengths was chosen based on the absorption wavelengths.

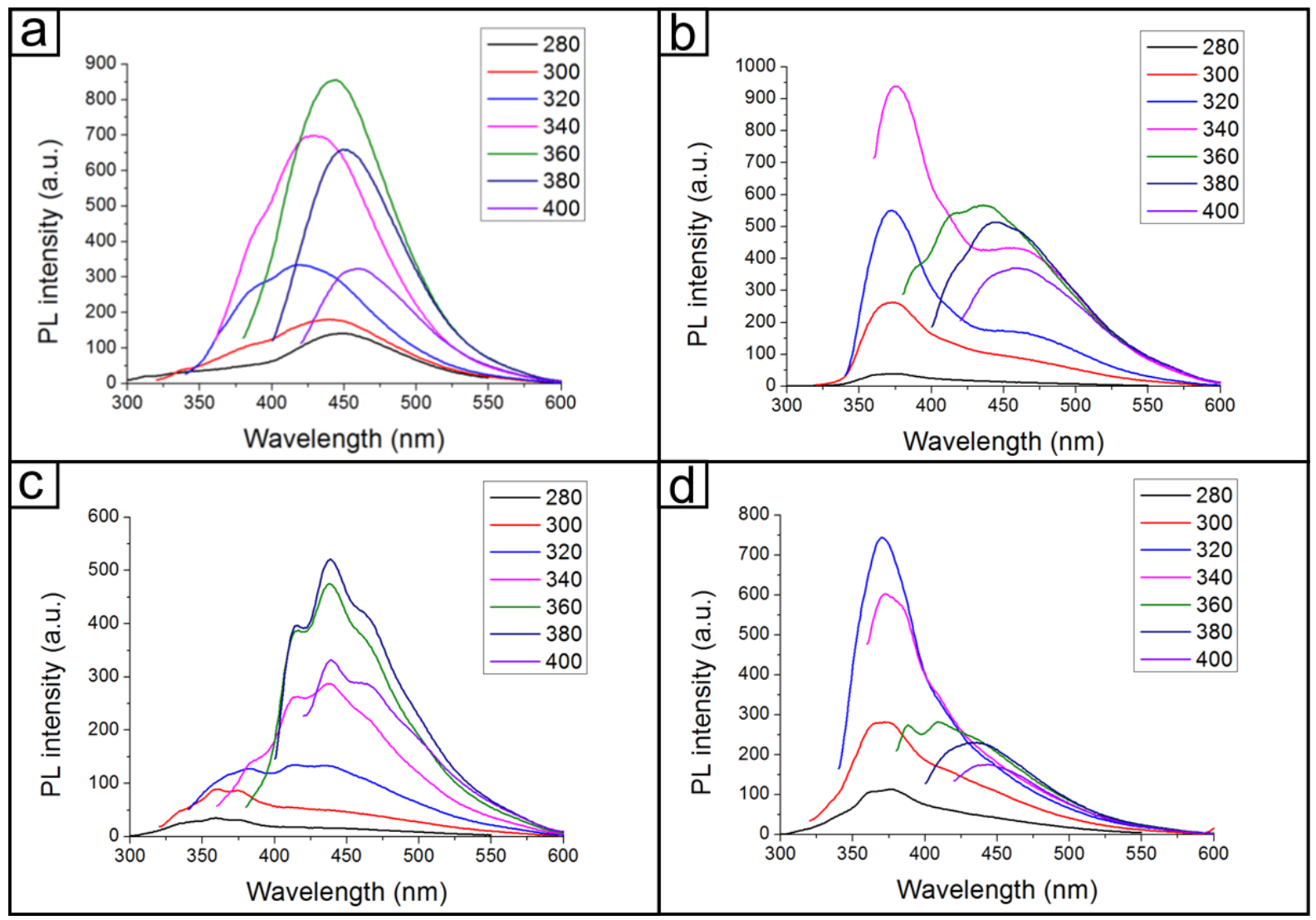

Figure 5.3: Photoluminescence spectra for germanium nanocrystals terminated with a) allylamine, b) epoxy, c) dodecene and d) diol. 
The four different terminated germanium quantum dots present different photoluminescence peak maxima. All four capping molecules have an emission range between 300 and $600 \mathrm{~nm}$. However, it is noticeable that peak features are significantly different for the four samples. Amine and dodecene terminated GeQDs present a main PL peak at $450 \mathrm{~nm}$, while epoxy and diol terminations lead to a maximum PL emission at $380 \mathrm{~nm}$. Epoxy-, diol- and dodecyl- ligands present a multitude of peaks with the same emission wavelength but different intensities. The solvent is not a factor between those three samples, so we here demonstrate the influence of the capping agent. The multi peak emission process is evidence of a multi electronic transition. ${ }^{19}$ These results cannot be explained only in terms of the quantum confinement effect. The direct band gap theory is a too simplistic approach and does not explain the variations with different capping agents. This study reveals a strong influence of the surface effects, not exclusively due to the solvent environment. This observation is in good agreement with Sakka et al and Shiohara et al with silicon quantum dots and recently by Wheeler et al for germanium quantum dots. ${ }^{20-22}$

A photobleaching study evaluates the chemical degradation of a dye or chromophore under light exposure over time. We compare the degradation of our germanium quantum dots with different surface capping to the photodegradation of tryptophan in solution, a commercially available dye. The dye was chosen for its emission wavelength as it is in the same range as GeQDs. ${ }^{23,24}$ A comparison of the absorption of the germanium quantum dots with the four different capping molecules to the one of tryptophan can be found in appendix 1 . The photobleaching trend of these species is obtained by the area under the photoluminescence curve of the different species over time. The concentration was kept constant for all the samples. The emission was recorded every 5 minutes for one hour for each sample. The normalized integrated intensity over time is represented in figure 5.4. 


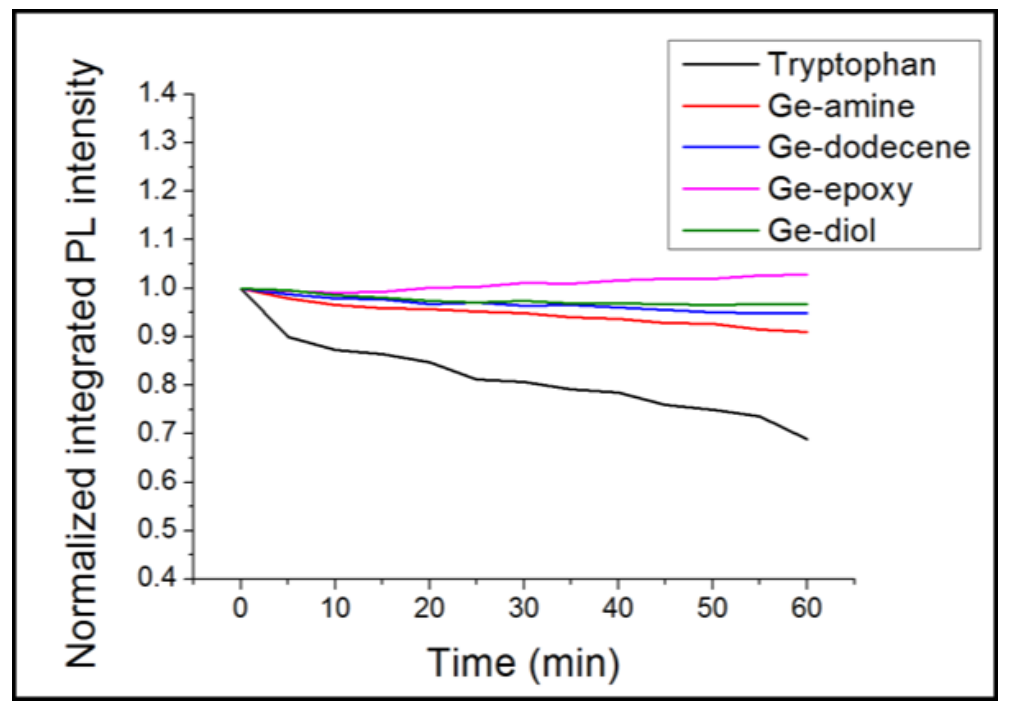

Figure 5.4: Photobleaching study comparing the decay of the integrated photoluminescence of GeQDs with amine, dodecene, diol or epoxy capping compared to Tryptophan.

The plots of the photoluminescence intensity over time clearly show a diminution of the photoluminescence intensity of tryptophan. The decrease corresponds to a $34 \%$ loss of photoluminescence after one hour of light exposure, which is the close to the degree of photodegradation reported in the literature. ${ }^{25}$ The photoluminescence of germanium quantum dots change less over time. Allylamine capped germanium nanocrystals are the ones presenting the largest decrease of intensity with a 9\% decrease. GeQDs-dodecene suffer from a decrease of fluorescence intensity of $5 \%$ and GeQDs terminated with a diol group lose about 3\% of their PL intensity. GeQDs terminated with an epoxy group show a $2 \%$ increase of intensity that can be due to a measuring error or a photoactivation of surface sites on the surface of germanium quantum dots. These observations confirm the better resistance to photodegradation of quantum dots compared to conventional organic dyes. ${ }^{26}$ The slight decrease of PL intensity observed dodecyl-, diol- or amineterminated germanium quantum dots is probably due to the degradation of the surface ligand, creating oxygen defects on the surface, and thus quenching the photoluminescence. ${ }^{27}$

The efficiency of the photoluminescence is defined by the quantum yield (QY). The quantum yield is defined as the ratio of the number of photons emitted to the number of photons absorbed. The photoluminescence intensity is recorded for five amine and 
dodecyl terminated germanium quantum dots solutions in water with decreasing concentration for an excitation wavelength of $360 \mathrm{~nm}$ for amine capping and $340 \mathrm{~nm}$ for dodecene capping.

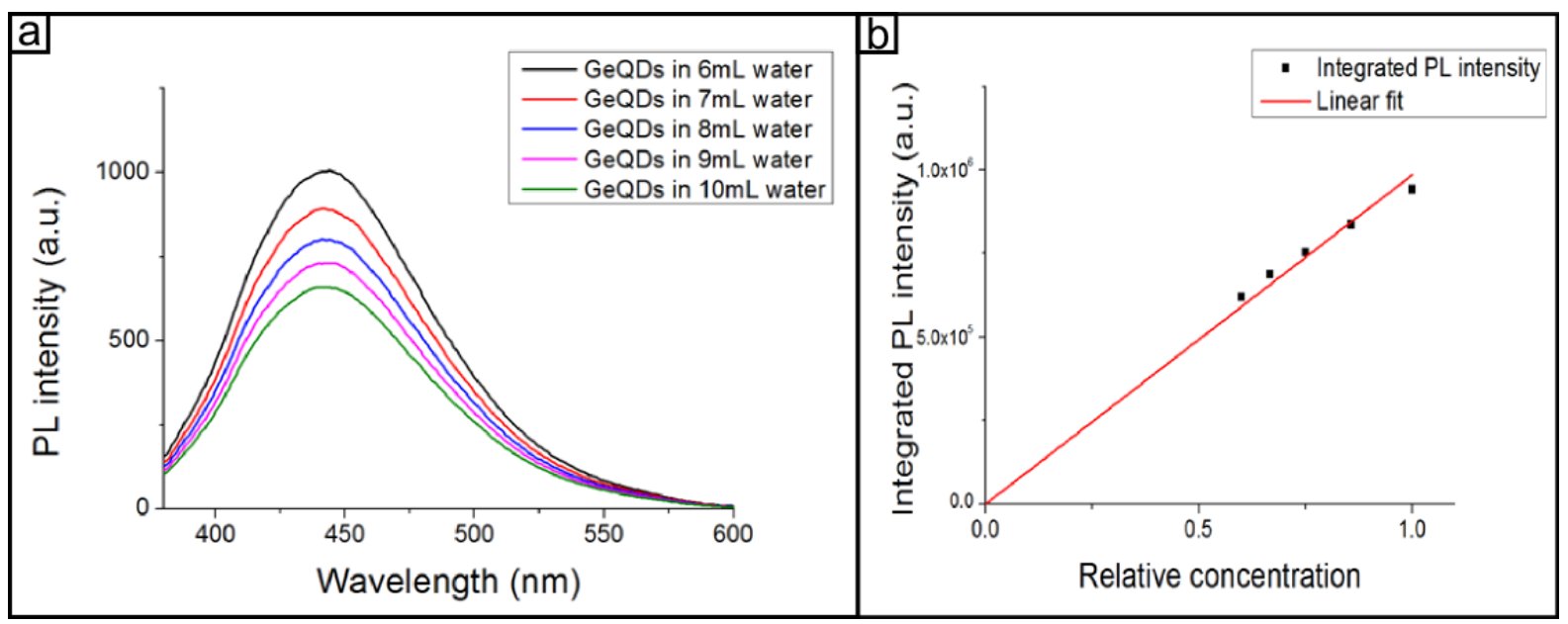

Figure 5.5: Quantum yield measurements for amine terminated germanium quantum dots.

The quantum yield was measured for our amine terminated germanium quantum dots by comparing the relative slope of the integrated PL intensity over the relative concentration to the standard tryptophan in water. A quantum yield of $16 \%$ is obtained for our germanium quantum dots in water. Similar experiment has been conducted on alkene terminated germanium quantum dots solution in chloroform and we obtained a quantum yield of $11 \%$. The highest quantum yield for blue-green emitting germanium nanocrystals of $17 \%$ has been reported by the Shiharata group in $2013 .{ }^{28}$ Previously in the Tilley group, Prabakar reported a QY of amine terminated GeQDs of $11 \%$ at 400 $n m .{ }^{29}$ The difference in $Q Y$ value between this research and the previous research is explained by the fact that GeQDs emit poorlier at 400 than 360, as seen in figure 5.3 a).

The lower QY value for alkene terminated GeQDs compared to the amine terminated ones can be associated to the influence of the surface chemistry or to the use of a different solvent from water (amine-GeQDs) to chloroform (alkene-GeQDs). We can then conclude that there is a great influence of the surface chemistry of germanium nanocrystals and also the solvent environment. ${ }^{30}$ 


\subsection{Synthesis of tin nanoparticles via chemical reduction}

This section describes the synthesis of tin nanoparticles. This research has been carried out in an attempt to synthesize nanocrystals of the semiconducting, also called $\alpha$, phase of tin. So far, only metallic $\beta$-tin has been successfully synthesized via a solution process. Several synthetic methods for $\beta$-tin can be found in the literature. Huang et al reported the fabrication of $\beta$-phased tin nanocrystals in a silicon nitride matrix by using a cosputtering technique. ${ }^{31}$ Li et al prepared tin nanoparticles form bulk via ultrasonic dispersion. ${ }^{32}$ Lai et al reported a thermal evaporation process and Zou et al synthesized $\beta$-tin nanocrystals by chemical reduction using sodium borohydride. ${ }^{33,34}$ To this day, the $\alpha$-phase of tin has been achieve only by physical deposition methods. Wang et al reported the cubic phase of tin confined in a nanotube of tin oxide synthesized using molecular beam epitaxy. ${ }^{35}$ They observed an increase of the maximum stability temperature of the diamond cubic phased tin from $13.2^{\circ} \mathrm{C}$ in the bulk form to up to $700^{\circ} \mathrm{C}$. The stabilization of $\alpha$-tin has also been reported by Asom et $a l$ in the thin film form, deposited onto InSb substrates by molecular beam epitaxy. ${ }^{36}$ The diamond cubic $\alpha$-tin nanoparticles are expected to present great semiconducting abilities. As the bulk $\alpha$-tin present a $0.08 \mathrm{eV}$, an infrared photoluminescence emission would be expected. For smaller nanoparticles, if the nanoparticles enter the quantum confinement regime, it would be expected an increase in the bandgap and consequently a blue shift of the PL emission, making $\alpha$-tin quantum dots ideal candidates for solar cells applications.

Unlike silicon or germanium, tin nanoparticles do not require a surface treatment. A thin $\mathrm{SnO}_{2}$ oxide shell is spontaneously formed by exposure to air. 


\subsubsection{At room temperature}

First will be investigated the formation of the tin nanoparticles via the microemulsion process developed by the Tilley group for silicon and germanium quantum dots. The reaction takes place at room temperature, but the confinement in a nanoparticle size could extend the stability of the diamond cubic structure of semiconducting tin at temperatures higher than $13.2^{\circ} \mathrm{C}$.

\section{Experimental}

$0.3 \mathrm{~g}$ of $\mathrm{C}_{12} \mathrm{E}_{5}$ was placed in a Shlenk tube. The reaction vessel was freed of oxygen by being put under vacuum for 2 minutes followed by being purged with nitrogen for 2 minutes. The process is repeated three times. Then, $30 \mathrm{~mL}$ of anhydrous toluene is injected into the tube through a septum seal. After 10 minutes of active stirring to allow the surfactant to form reverse micelles, $1.5 \mathrm{~mL}$ of $\mathrm{SnCl}_{4}$ solution in dichloromethane was added to the mixture and stirred for $1 \mathrm{~h}$. Lithium borohydride $(2.5 \mathrm{~mL})$ was then injected into the solution. The colour of the solution turns from slightly pink to grey. After $1 \mathrm{~h}$, the reaction was stopped and the nanoparticles obtained were separated from solution by centrifugation and washed by a mixture methanol/toluene.

\section{Characterization}

The crystalline structure of the synthesized nanoparticles was assessed using X-ray diffraction. The spectrum obtained is presented in figure 5.6. It presents well defined peaks that can be assigned to the tetragonal structure of the $\beta$-tin. Only the metallic phase of tin is present on the sample. 


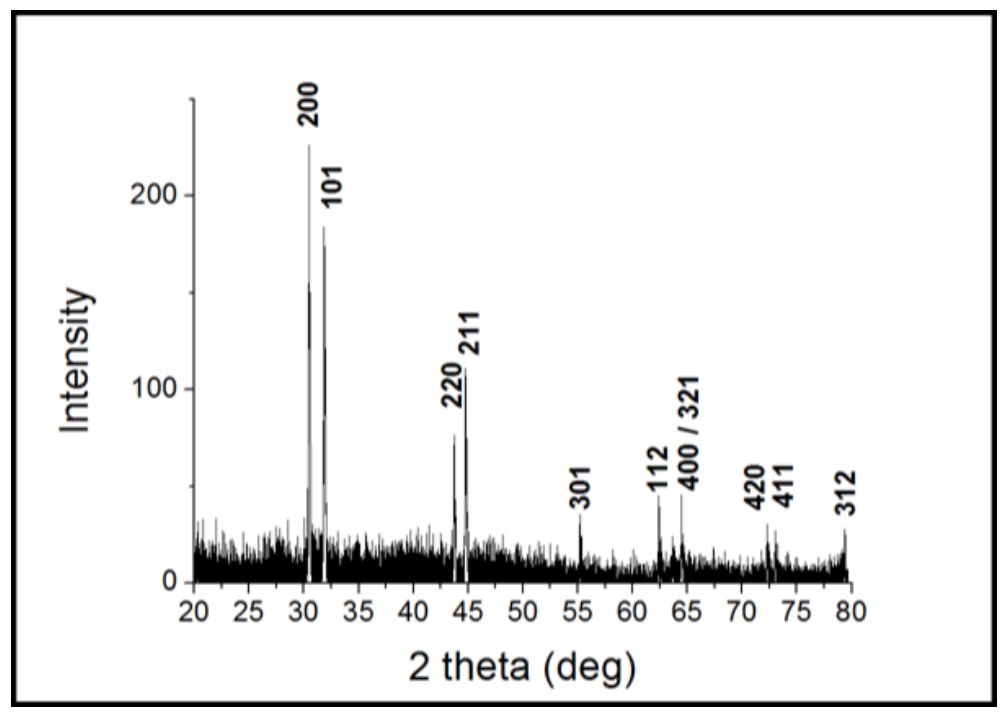

Figure 5.6: XRD pattern of tin nanoparticles obtained by chemical reduction at room temperature

The size and shape of the metallic tin nanoparticles is assessed with transmission electron microscopy. Figure 5.7 shows the TEM and HRTEM images, along with the size distribution diagram and the SAED pattern.

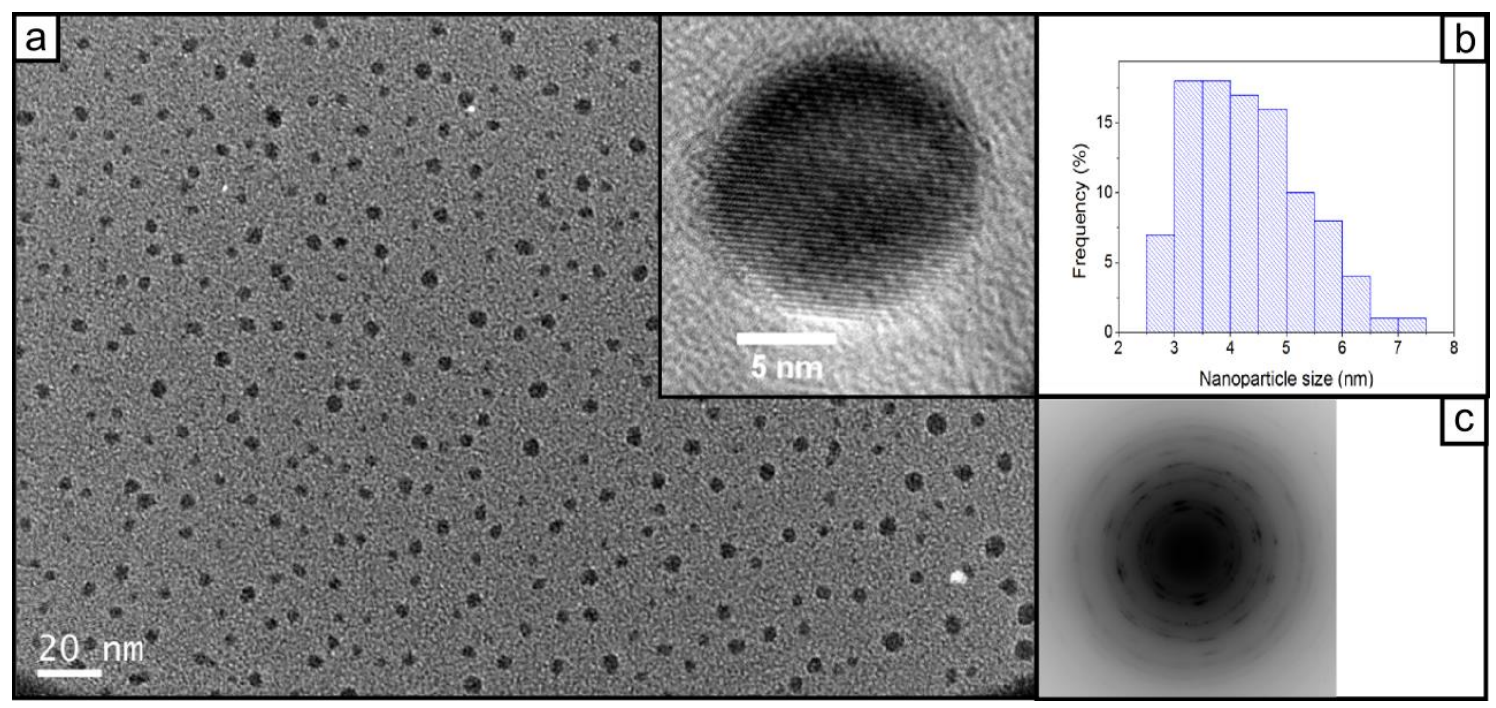

Figure 5.7: a) TEM image of tin nanocrystals obtained by chemical reduction at room temperature with an inset of a HRTEM image of one nanocrystal, b) the corresponding size distribution and c) the SAED diffraction pattern 
The TEM image presented in figure 5.7 shows that the tin nanoparticles are spherical in shape. The crystals appear to be also regular in size. The average size distribution is $4.3 \pm$ $2.4 \mathrm{~nm}$. The inset in figure 5.7.a depicts a high resolution TEM image of one nanocrystal. The lattice fringes observed attest of the monocrystalline nature of the nanoparticles. The distance between fringes of $0.32 \mathrm{~nm}$ corresponds to the (200) plane orientation of $\beta$-tin structure. SAED rings can be matched to the tetragonal phase of metallic tin.

Metallic tin has several potential applications as a low melting temperature metal with electron conductive properties. ${ }^{37,38}$ To exploit this conduction, tin nanoparticles need to be coatable on a surface. I therefore investigated the capacity of $\beta$-tin nanoparticles to form regular thin films using SEM imaging. To prepare the specimen, the nanocrystals solution in ethanol was drop casted onto the silicon wafer placed on a hot plate heated at $60^{\circ} \mathrm{C}$. The nanocrystals appear to be regular in size and are distributed evenly across the surface of the silicon wafer. Figure 5.8 represents the SEM image of the tin nanocrystals deposited onto a silicon wafer.

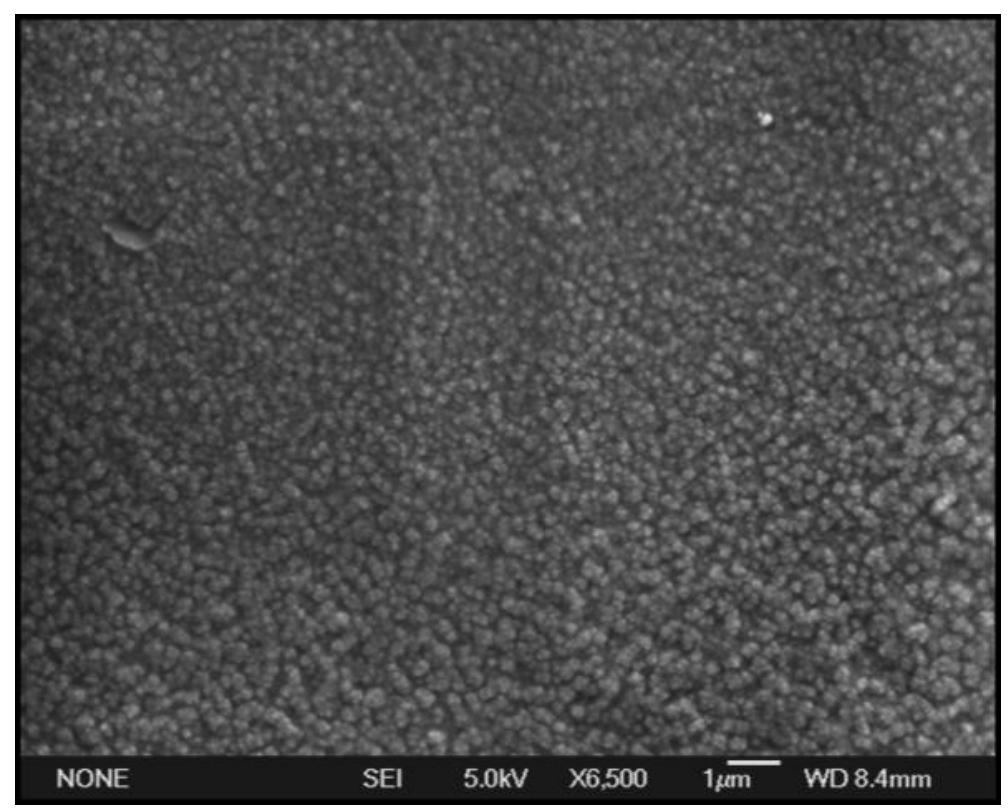

Figure 5.8: SEM image of tin nanocrystals deposited onto a silicon wafer.

The $\beta$-tin nanocrystals cover the majority of the surface of the silicon wafer. The nanoparticle film thickness does not appear regular over the entire surface of the wafer, most likely due to the method of deposition. For a more regular thickness, spin casting 
will be a more reliable method. Tin nanoparticles can easily be coated over a surface and have possible applications as low melting temperature soldering materials. ${ }^{37}$

\subsubsection{Reverse micelle synthesis in an ice-bath}

The $\alpha$-phase of tin is stable below $13^{\circ} \mathrm{C}$. The motivation for this work was to decrease the temperature of the nanoparticle synthesis. The reaction mixture was cooled to $0^{\circ} \mathrm{C}$ using an ice bath. With the synthesis taking place at a temperature below the transition temperature between $\alpha$ and $\beta$-phases of tin, it is expected to form pure $\alpha$-tin nanocrystals.

\section{Experimental}

The same experimental procedure as the one described in the paragraph 2.2.2 was performed in this experiment, but in this case the reaction Schlenk tube was placed in an ice bath.

\section{Characterization}

The crystal structure of the nanoparticles formed by chemical reduction of tin precursor in a solvent media cooled to $0^{\circ} \mathrm{C}$ is determined by X-ray diffraction. The XRD pattern is presented in figure 5.9.

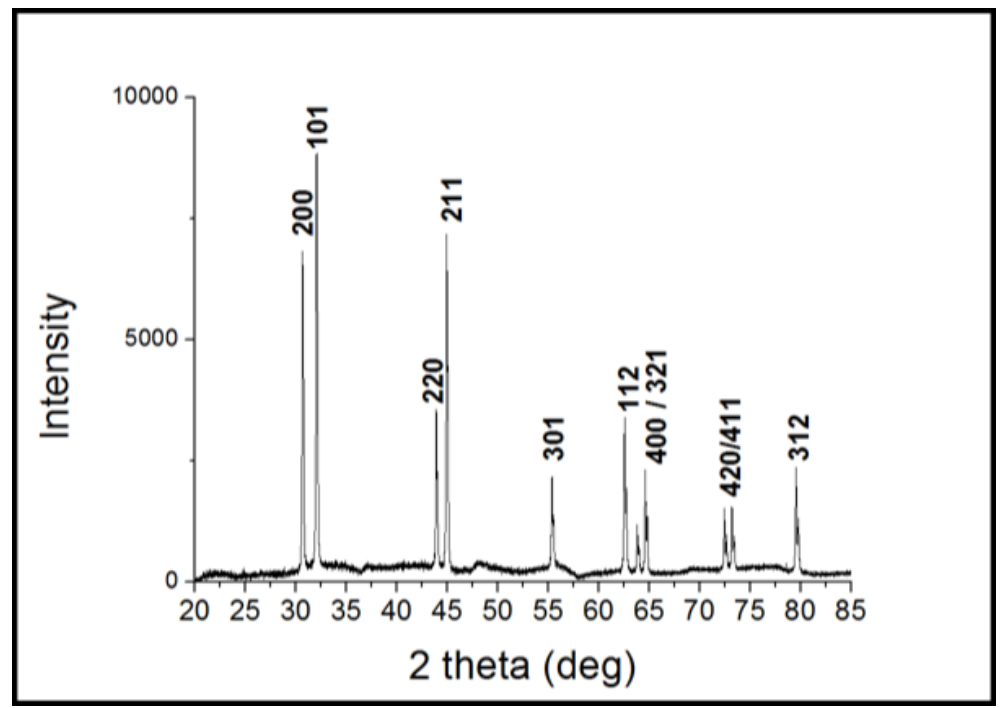

Figure 5.9: XRD pattern obtained from the tin nanoparticles synthesized by chemical reduction at $0^{\circ} \mathrm{C}$ 
The XRD spectrum presents well defined peaks correspong to only the $\beta$-tin crystal structure. Reducing the reaction temperature did not produce the $\alpha$-phase of tin. A possible explanation is that the chemical reduction with lithium borohydride is an exothermic reaction and the local temperature around the newly formed tin monomers might have been higher than the $13.2^{\circ} \mathrm{C} \alpha-\beta$ transition temperature.

The size and shape of the metallic tin nanoparticles is assessed with transmission electron microscopy. Figure 5.10 shows the TEM and HRTEM images, along with the size distribution diagram and the SAED pattern.

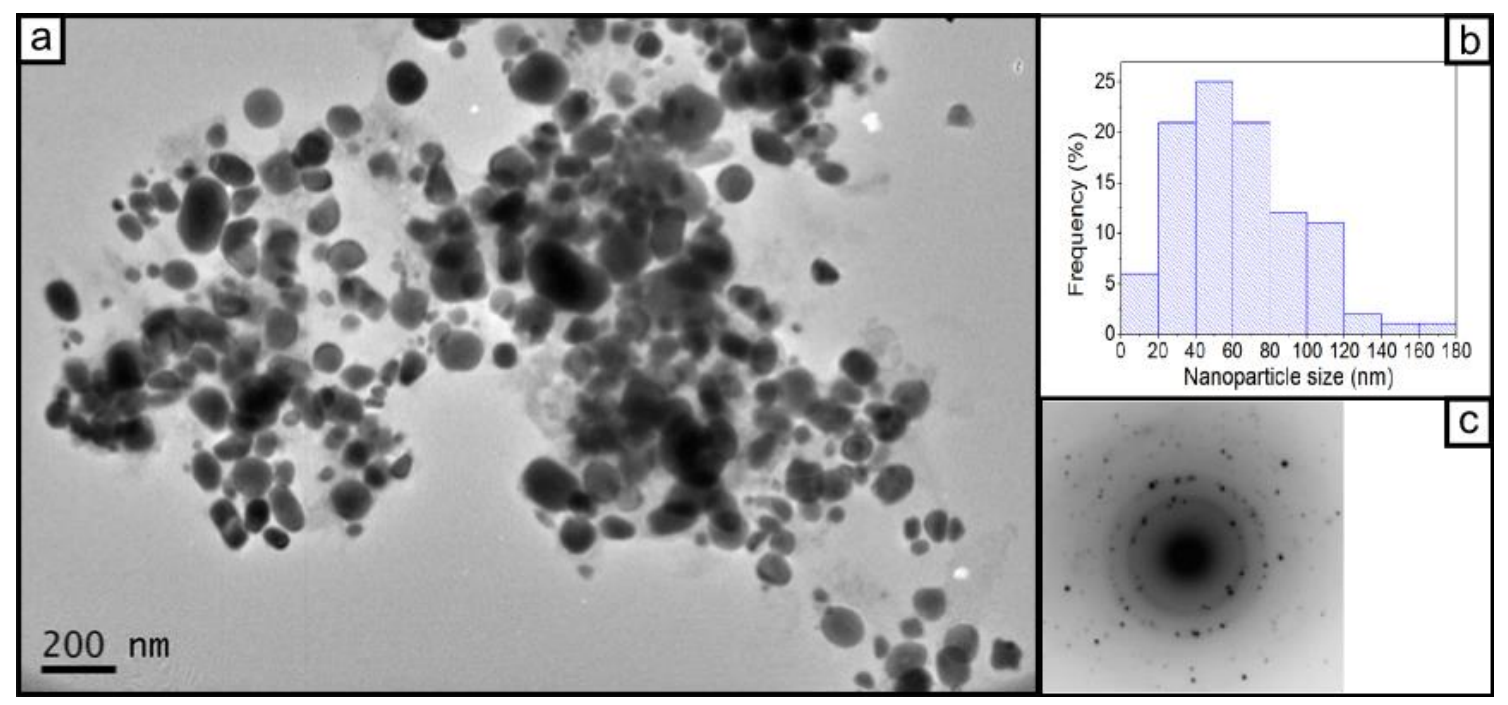

Figure 5.10: a) TEM image of tin nanoparticles synthesized at $0^{\circ} \mathrm{C}$ with b) the corresponding size distribution diagram and $c$ ) the SAED rings

The nanoparticles are presenting spherical shaped particles and more faceted shapes than the tin nanoparticles synthesized at room temperature. Also the average size is $62.4 \pm 78.3 \mathrm{~nm}$, which is much larger than the particles produced using the method in section 5.2.1. This size increase can be explained by a slower chemical reduction process due to the lower temperature. As the reduction took longer, the nucleation process was extended, allowing particles to grow more slowly, hence producing a larger size distribution. 
The ability of those tin nanocrystals to form a regular film over a flat surface was investigated using scanning electron microscopy. A nanocrystals solution in ethanol was drop casted onto a silicon wafer heated up to $60^{\circ} \mathrm{C}$ on a hot plate. The SEM image of the coated silicon wafer is shown in figure 5.11. The surface of the wafer was fully covered by nanocrystals.

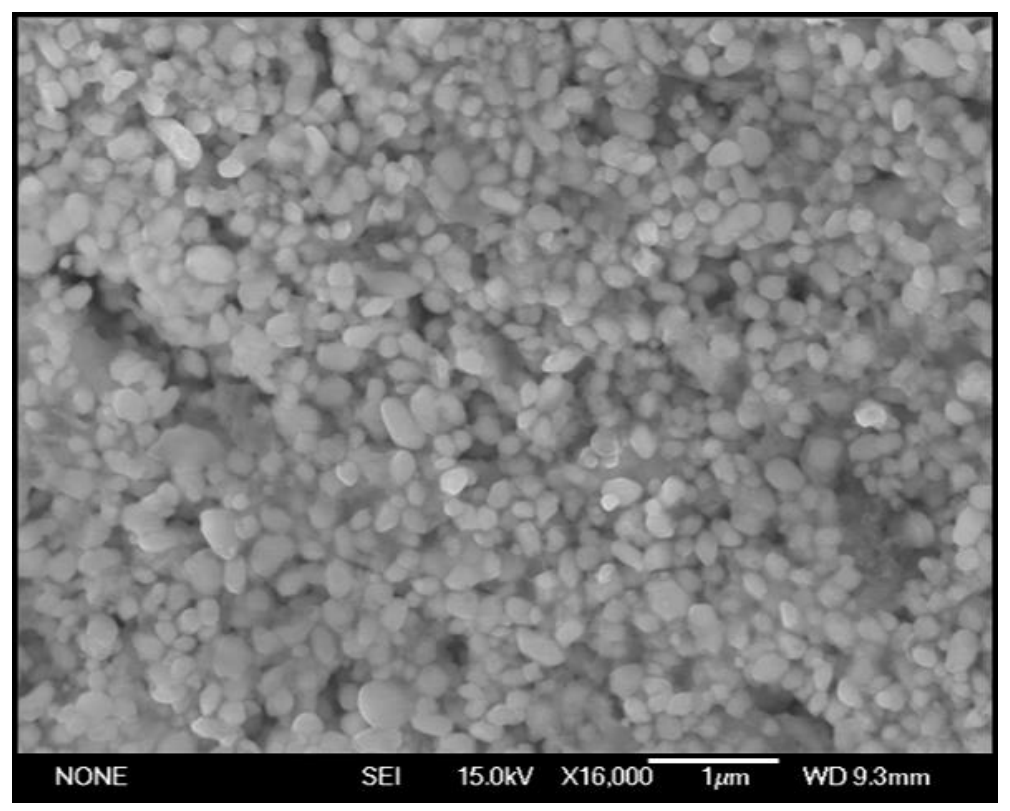

Figure 5.11: SEM image of tin nanocrystals synthesized at $0^{\circ} \mathrm{C}$ deposited onto a silicon wafer.

The metallic tin nanoparticles form a relatively smooth film over the surface of the silicon wafer. Such is required for applications such as soldering or for use as a conductive ink. ${ }^{37,38}$ The ease of processing makes tin nanoparticles a suitable candidate for large scale device fabrication such as roll-to-roll processing.

\subsection{Alloying tin with germanium}

A possible way to stabilize the $\alpha$-tin structure at temperatures higher than $13.2^{\circ} \mathrm{C}$, the $\alpha$ $\beta$ transition temperature at ambient pressure, would be to alloy tin with another element with a diamond-type cubic structure. ${ }^{10}$ Silicon and Germanium spontaneously 
crystallise in the diamond cubic form and some examples of successful alloy with tin have been reported in the literature. Tin-germanium alloy is expected to have semiconducting properties. ${ }^{39}$ The $\mathrm{Sn}-\mathrm{Ge}$ solid solution presents a low solubility of germanium into tin. King et al stated a maximum concentration of $1 \%$ of germanium into tin. ${ }^{40}$ This low solubility is probably due to the large lattice difference between the two elements, which are $5.646 \AA$ for $\mathrm{Ge}$ and 6.489 for $\alpha$-tin and to the fact that at ambient conditions of temperature and pressure, the cubic-phase of tin is unstable. Interestingly, the nature of the band gap changes with the concentration of tin in the solid solution $\mathrm{Ge}_{1-\mathrm{x}} \mathrm{Sn}_{\mathrm{x}}$. At very low concentrations $(\mathrm{x}<0.25)$, the band gap transition is indirect, as the band gap of bulk germanium, but with increasing tin concentration, the band gap becomes direct. ${ }^{39}$ Tin-rich alloys are far less common that germanium rich tingermanium alloys. Pukite et al reported a single phase $\mathrm{Sn}_{x} \mathrm{Ge}_{1-\mathrm{x}}$ grown by molecular beam epitaxy, with $x$ up to a value of $0.3 .^{41}$ They observed that above this concentration of $30 \%$ of tin, the film causes phase separation into the tetragonal $\beta$-tin phase. Kouvetakis et al. developed the growth of $\mathrm{Ge}_{1-x} \mathrm{Sn}_{x}$ alloy, with $5 \%<x<25 \%$, by chemical vapour deposition. ${ }^{10}$ They stated a high thermal stability of the cubic structure up to $600^{\circ} \mathrm{C}$. The diffusion of germanium in tin has also been used for the growth of germanium nanowires, using tin nanoparticles as seeds. It appears that no solution phase synthesis of Sn-Ge alloy with a diamond cubic structure has been reported in the literature. 


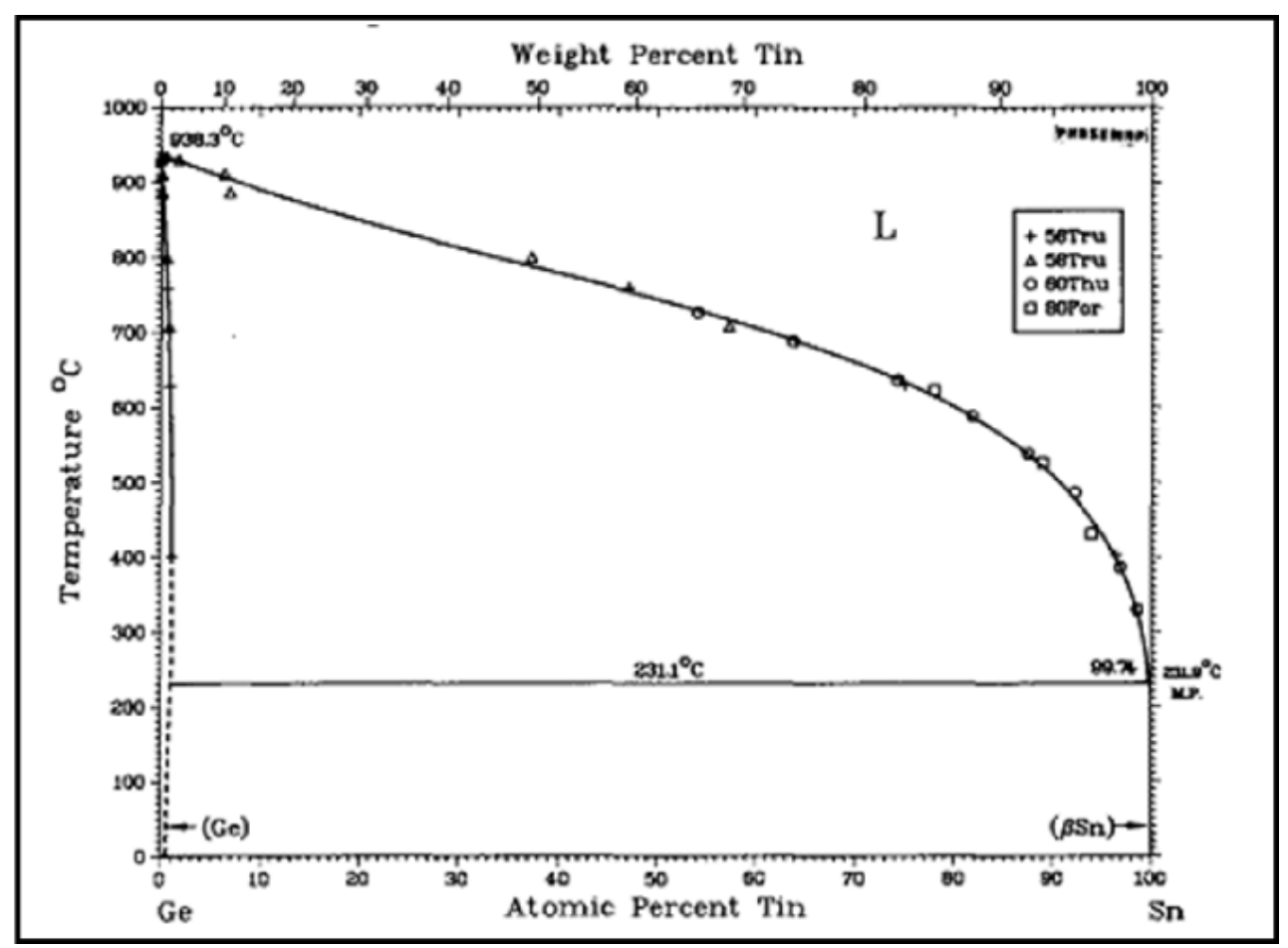

Figure 5.12: Phase diagram for the Ge-Sn( $\beta)$ system, reproduced from Olesinki et al. ${ }^{42}$

The phase diagram reported by Olesinki et al shows a less than $1 \%$ solubility of Ge into $\beta$-tin. There is no phase diagram of $\mathrm{Ge}-\mathrm{Sn}(\alpha)$ reported in the literature. It is anticipated that the introduction of germanium as a dopant element in the tin structure to stabilize tin into the diamond cubic structure corresponding to the semiconducting phase of tin due to the similar crystal structure of germanium. Several levels of germanium doping have been investigated.

\subsubsection{Alloying tin and germanium using chemical reduction}

\section{Experimental}

For all five samples, a Schlenk tube containing $0.3 \mathrm{~g}$ of $\mathrm{C}_{12} \mathrm{E}_{5}$ was placed under nitrogen atmosphere. $30 \mathrm{~mL}$ of toluene was injected in the tube through a Septum seal. After 10 minutes of active stirring, $1.56 \mathrm{~mL}$ of $\mathrm{SnCl}_{4}$ and $1.8 \mu \mathrm{L}$ of $\mathrm{GeCl}_{4}$ were injected simultaneously into the solution. The mixture was stirred vigorously for $1 \mathrm{~h}$. $2.5 \mathrm{~mL}$ of $\mathrm{LiBH}_{4}$ solution were then injected into the tube. The solution turned grey. The particles were separated from solution using centrifugation and were then purified by washing using toluene/methanol mixture. 


\section{Characterization}

The crystal structure of the tin-germanium alloy nanoparticles formed by chemical reduction is determined by XRD. The patterns for $1 \%, 2 \%, 5 \%, 10 \%$ and $50 \%$ of germanium in the alloy are presented in figure 5.13 and represent the attempted $\mathrm{Sn}_{0.99} \mathrm{Ge}_{0.01}, \mathrm{Sn}_{0.98} \mathrm{Ge}_{0.02}, \mathrm{Sn}_{0.95} \mathrm{Ge}_{0.05}, \mathrm{Sn}_{0.9} \mathrm{Ge}_{0.1}$ and $\mathrm{Sn}_{0.5} \mathrm{Ge}_{0.5}$ alloys.

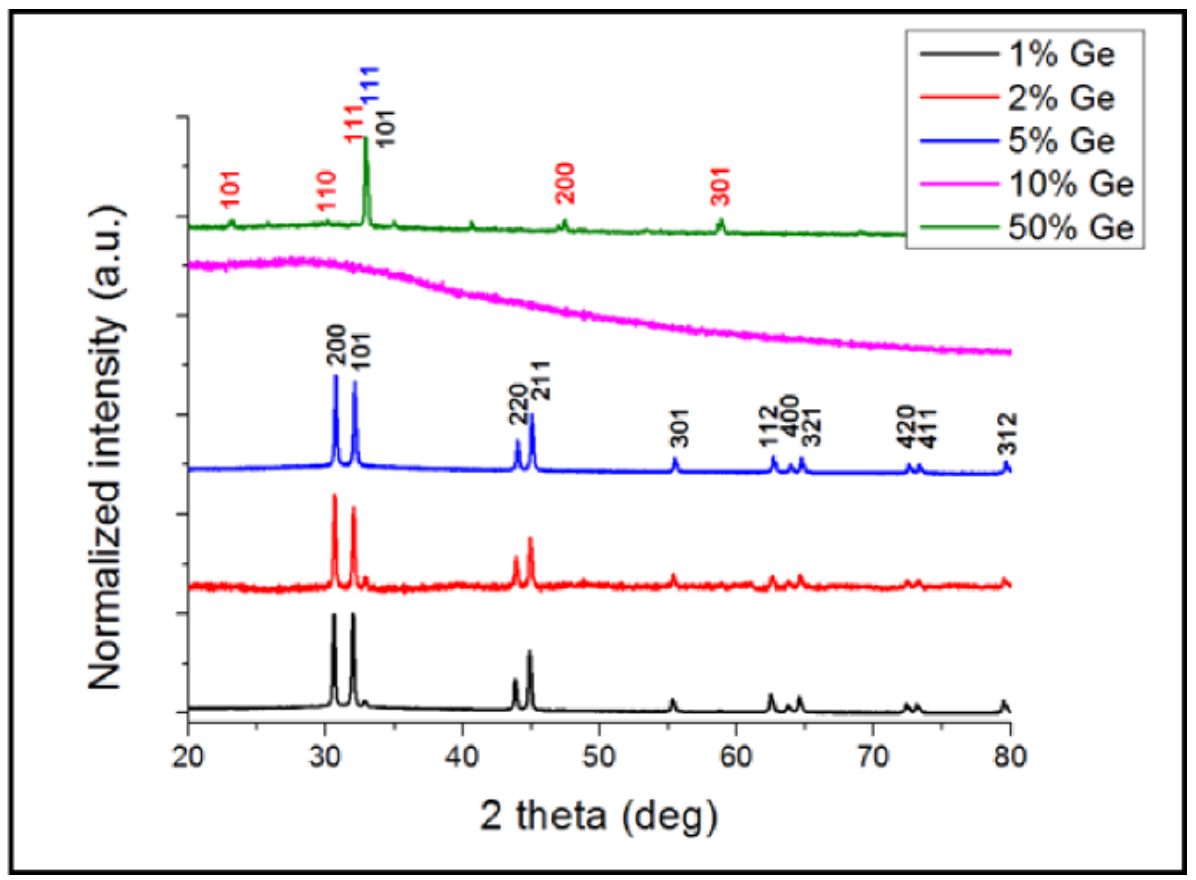

Figure 5.13: XRD pattern for nanoparticles obtained for $1 \%, 2 \%, 5 \%, 10 \%$ and $50 \%$ of germanium in the alloy. Reflections corresponding to $\beta$-tin (JCPDS 18-1380) are marked

in black, germanium reflections are represented in red (JCPDS 77-6203) and $\alpha$-tin reflection is represented in blue (JCPDS 13-6167).

Samples synthesized with 1, 2 and $5 \%$ present a pure $\beta$-tin tetragonal structure. Using $10 \%$ of germanium, the nanoparticles formed were amorphous and no diffraction peaks were observed. When trying to alloy $50 \%$ Sn with $50 \% \mathrm{Ge}$, the XRD pattern is dominated by the germanium crystal structure. The main peak at $2 \Theta=33^{\circ}$ can also be associated to the main reflection of $\alpha-S n$ and to the (101) orientation of $\beta-S n$. It cannot be concluded to the successful synthesis of the $\alpha$-Sn semiconducting phase.

The size and shape of the nanoparticles is assessed with transmission electron microscopy. Figure 5.14 shows the TEM images obtained for the four crystalline nanoparticles obtained: $1 \%, 2 \%, 5 \%$ and $50 \%$. 


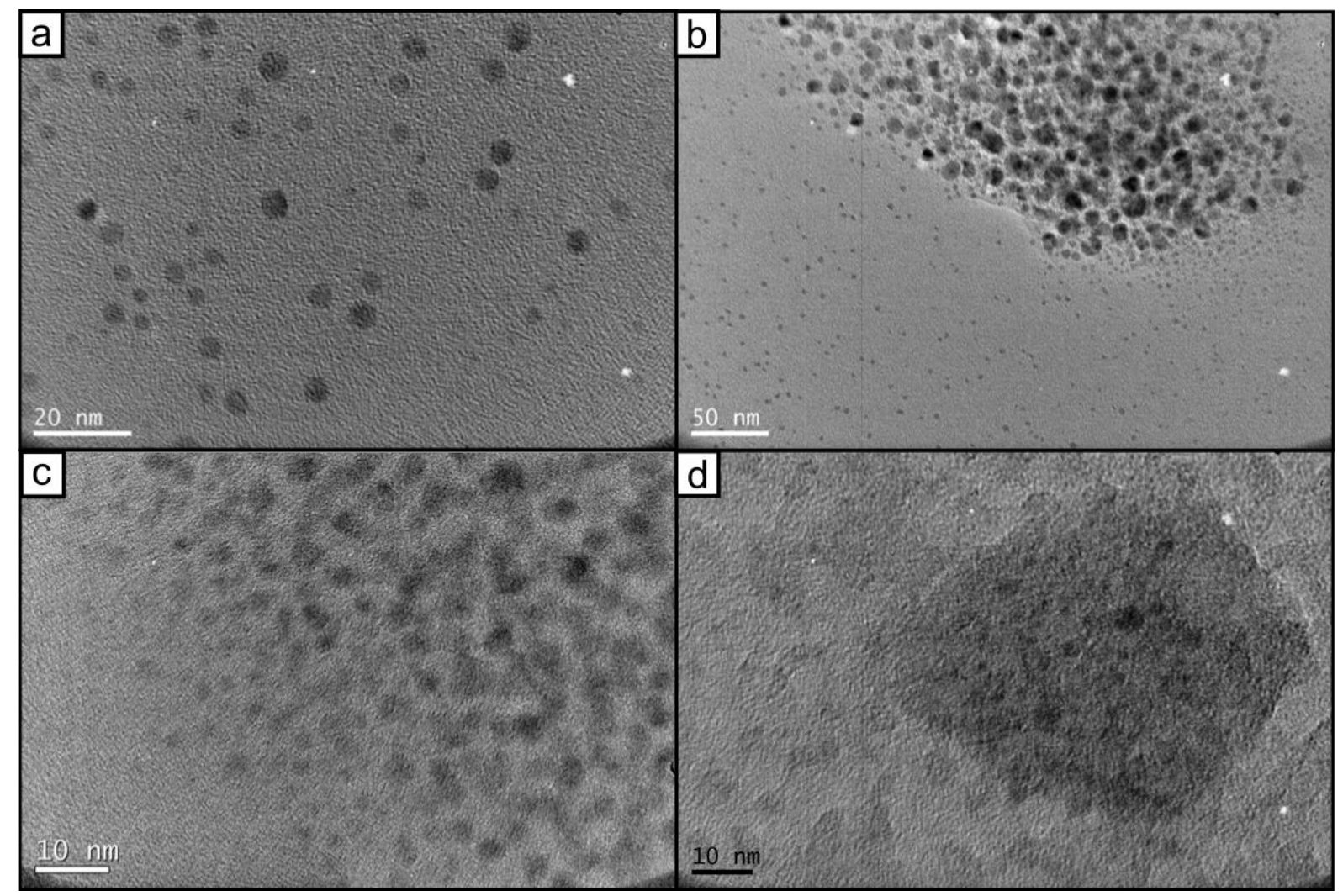

Figure 5.14: TEM images for SnGe alloys nanoparticles with a) $1 \% \mathrm{Ge}$, b) $2 \% \mathrm{Ge}$, c) $5 \% \mathrm{Ge}$ and d) $50 \% \mathrm{Ge}$

Alloys with $1 \%$ and $5 \%$ of germanium into tin formed nanoparticles with an average size below $10 \mathrm{~nm}$. Alloys with $2 \%$ germanium present two types of particles, some below 10 $\mathrm{nm}$ in diameter and some bigger particles $(15-25 \mathrm{~nm})$ that appear to be agglomerated. Figure 5.14.d show particles obtained for the 50-50 ratio of precursors. Even if the sample still present surfactant impurities, particles exhibit an average size below $10 \mathrm{~nm}$.

\subsubsection{Alloying tin with germanium using temperature decomposition}

As the chemical reduction method was not successful in producing SnGe alloys with a diamond cubic structure, attention was focused on high temperature decomposition methods.

\section{Experimental}

In a three necked flask under nitrogen atmosphere, $0.33 \mathrm{~mL}$ of $1 \mathrm{M} \mathrm{SnCl}_{4}$ in dichloromethane is added to $10 \mathrm{~mL}$ of trioctylamine. In a separate flask, $4 \mu \mathrm{L}$ of $\mathrm{GeCl}_{4}$ is 
mixed with $2 \mathrm{~mL}$ of oleylamine. The three-necked flask is heated at $230^{\circ} \mathrm{C}$, then the germanium solution is swiftly injected and the mixture is heated to $300^{\circ} \mathrm{C}$ for 4 hours. The mixture was left to cool naturally to room temperature. The particles were separated by centrifugation then washed with a mixture methanol/toluene.

\section{Characterization}

The crystal structure of the tin-germanium alloy nanoparticles formed by chemical reduction is determined by XRD. The patterns for $5 \%$ and $10 \%$ of germanium in the alloy are presented in figure 5.15 .

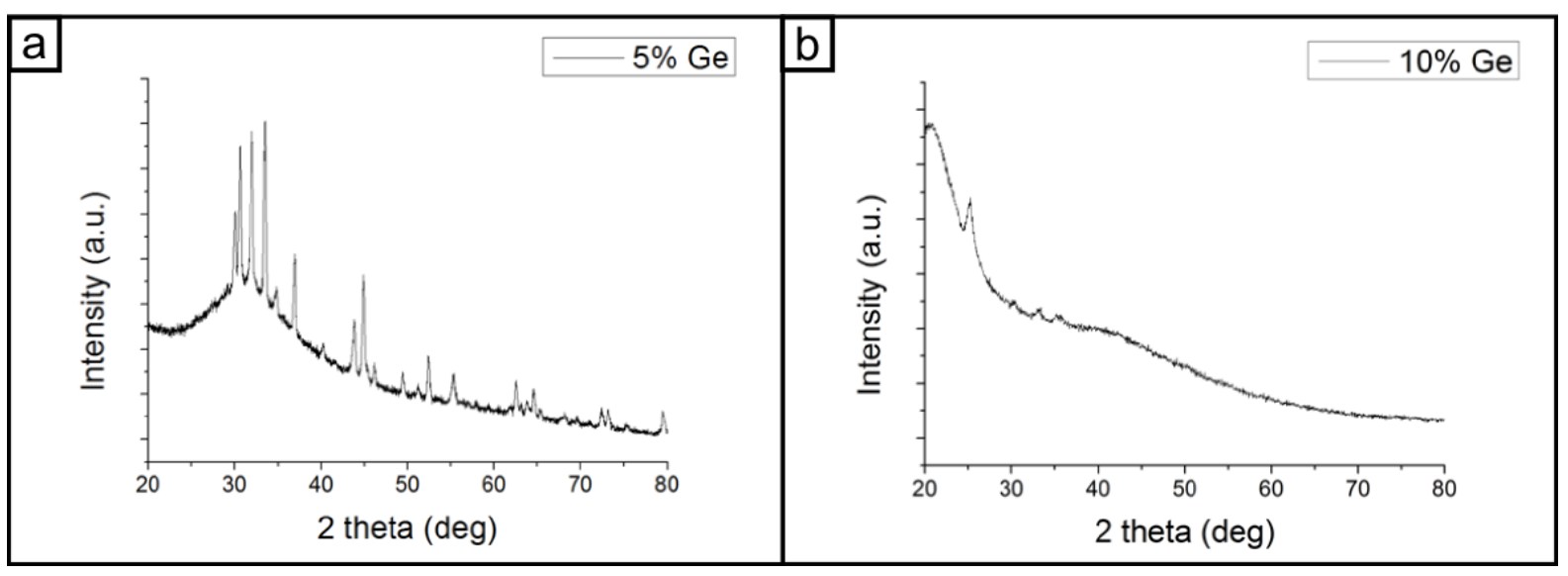

Figure 5.15: XRD spectrum for nanoparticles obtained after high temperature decomposition with a) $5 \% \mathrm{Ge}$ and b) $10 \% \mathrm{Ge}$

The XRD spectrum of the nanocrystals obtained for the proportions $\mathrm{Sn}_{0.95} \mathrm{Ge}_{0.05}$ present two phases: the $\beta$-tin tetragonal structure and tin oxide. In previous experiments, the tin nanoparticles presented a thin shell of tin oxide, which structure was not appearing on XRD spectrum due to its small amount compared to the $\beta$-tin structure. The relative peak intensity of tin oxide compared to the $\beta$-tin structure lead to the conclusion that the particles are significantly oxidized. For $\mathrm{Sn}_{0.9} \mathrm{Ge}_{0.1}$ the large background noise suggest a large amount of amorphous material. As it can be seen on figure 5.15.b, the peak at $27^{\circ}$ corresponds to the germanium structure and the two peaks at $\Theta=33$ and $35^{\circ}$ correspond to the (200) and (101) orientations of $\beta$-tin crystalline form. 
The size and shape of the nanoparticles is assessed with transmission electron microscopy. Figure 5.16 shows the TEM and HRTEM images obtained for the two alloy nanoparticles: $\mathrm{Sn}_{0.95} \mathrm{Ge}_{0.05}$ and $\mathrm{Sn}_{0.9} \mathrm{Ge}_{0.1}$.

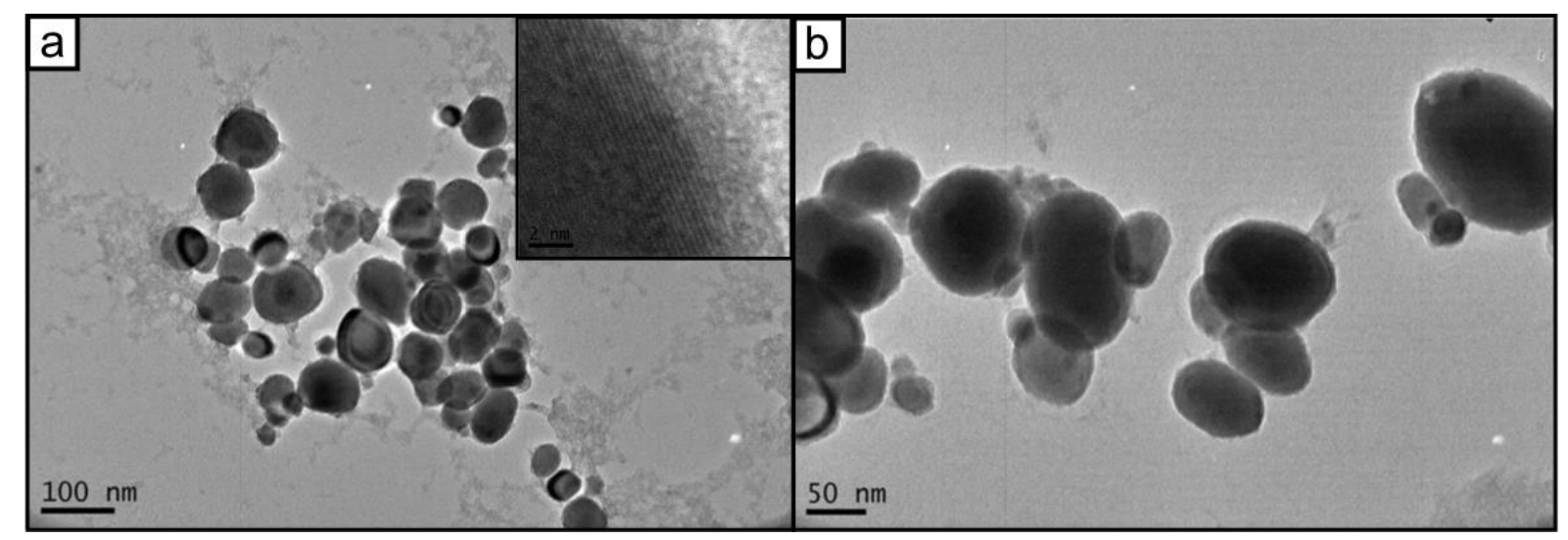

Figure 5.16: TEM images of tin/germanium alloy nanoparticles with a) $5 \%$ Ge and b) $10 \%$ $\mathrm{Ge}$

Particles synthesized via the temperature decomposition method are of much bigger size than $\mathrm{SnGe}$ alloy particles synthesized via chemical reduction. $\mathrm{Sn}_{0.95} \mathrm{Ge}_{0.05}$ particles measure $60 \mathrm{~nm}$ on average and as it can be seen in the inset of figure 5.16.a, a $4 \mathrm{~nm}$ thick oxide shell is observed on the particles.

A pure SnGe alloy phase was not achieved by synthesis in solution via high temperature decomposition of tin and germanium precursors. The particles obtained with this method have a much larger average size than the nanocrystals obtained by chemical reduction in reverse micelles. 


\subsection{Discussion}

Germanium nanocrystals with a narrow size distribution can be synthesized via the microemulsion synthetic process. The GeQDs capped with four different ligands present no structural difference, but present very different optical properties. The emission spectra are representative of a multi electronic transition process, but the emission range remains constant for all GeQDs with different capping molecules. This observation indicates the emission is from surface "trap" states rather than from the most commonly reported quantum confinement induced direct band gap theory. ${ }^{43}$

Metallic $\beta$-tin can be synthesized by chemical reduction of tin tetrachloride. The temperature of the solvent does not influence the crystal structure, but plays an important role in the average size of the nanocrystals. Cooling the reaction temperature to $0^{\circ} \mathrm{C}$ extends the nucleation time by slowing down the chemical reduction, thus forming larger $\beta$-tin nanoparticles than the homologue synthesis at room temperature.

Attempts to alloy tin and germanium did not lead to the stabilization of the diamond cubic structure of $\alpha$-tin. Via chemical reduction, alloys with 1, 2 or $5 \%$ of germanium present only the $\beta$-tin tetragonal structure. Starting with $50 \%$ germanium, the nanoparticles presents a mixture of tin and germanium structures, but there is no evidence that $\alpha$-tin structure has been achieved. 


\subsection{References}

1_ Kelly, J. A.; Henderson, E. J.; Veinot, J. G. C., Sol-gel precursors for group 14 nanocrystals. Chemical Communications 2010, 46 (46), 8704-8718.

2_Buriak, J. M., Organometallic Chemistry on Silicon and Germanium Surfaces. Chemical Reviews 2002, 102 (5), 1271-1308.

3_ Cullis, A. G.; Canham, L. T.; Calcott, P. D. J., The structural and luminescence properties of porous silicon. Journal of Applied Physics 1997, 82 (3), 909-965.

4_ Gresback, R.; Holman, Z.; Kortshagen, U., Nonthermal plasma synthesis of sizecontrolled, monodisperse, freestanding germanium nanocrystals. Applied Physics Letters 2007, 91 (9), 093119-3.

5_Wilcoxon, J. P.; Provencio, P. P.; Samara, G. A., Synthesis and optical properties of colloidal germanium nanocrystals. Physical Review B 2001, 64 (3), 035417.

6_Allan, G.; Delerue, C., Optimization of Carrier Multiplication for More Effcient Solar Cells: The Case of Sn Quantum Dots. ACS Nano 2011, 5 (9), 7318-7323.

7_ Wang, B.; Ouyang, G.; Yang, Y. H.; Yang, G. W., Anomalous thermal stability of cubic tin confined in a nanotube. Applied Physics Letters 2007, 90 (12), 121905-3.

8_Ragan, R.; Min, K. S.; Atwater, H. A., Direct energy gap group IV semiconductor alloys and quantum dot arrays in SnxGe1-x/Ge and SnxSi1-x/Si alloy systems. Materials Science and Engineering B 2001, 87 (3), 204-213.

9_Goodman, C. H. L., Band structure of $\alpha-S n$ and Ge-Sn alloys. Solid-State and Electron Devices, IEE Proceedings / 1984, 131 (3), 109-110. 
10_Taraci, J.; Tolle, J.; Kouvetakis, J.; McCartney, M. R.; Smith, D. J.; Menendez, J.; Santana, M. A., Simple chemical routes to diamond-cubic germanium--tin alloys. Applied Physics Letters 2001, 78 (23), 3607-3609.

11_Huang, S.; Cho, E.-C.; Conibeer, G.; Green, M. A.; Bellet, D.; Bellet-Amalric, E.; Cheng, S., Fabrication and characterization of tin-based nanocrystals. Journal of Applied Physics 2007, 102 (11), 114304-6.

12_Pukite, P. R.; Harwit, A.; Iyer, S. S., Molecular beam epitaxy of metastable, diamond structure $\mathrm{Sn}_{\mathrm{x}} \mathrm{Ge}_{1-\mathrm{x}}$ alloys. Applied Physics Letters 1989, 54 (21), 2142-2144.

13_Taylor, B. R.; Kauzlarich, S. M.; Delgado, G. R.; Lee, H. W. H., Solution Synthesis and Characterization of Quantum Confined Ge Nanoparticles. Chemistry of Materials 1999, $11(9), 2493-2500$.

14_Ma, X.; Wu, F.; Kauzlarich, S. M., Alkyl-terminated crystalline Ge nanoparticles prepared from NaGe: Synthesis, functionalization and optical properties. Journal of Solid State Chemistry 2008, 181 (7), 1628-1633.

15_Lu Lu, X.; Ziegler, K. J.; Ghezelbash, A.; Johnston, K. P.; Korgel, B. A., Synthesis of Germanium Nanocrystals in High Temperature Supercritical Fluid Solvents. Nano Letters 2004, 4 (5), 969-974.

16_Wu, H. P.; Liu, J. F.; Wang, Y. W.; Zeng, Y. W.; Jiang, J. Z., Preparation of Ge nanocrystals via ultrasonic solution reduction. Materials Letters 2006, 60 (7), 986-989.

17_Wilcoxon, J. P.; Provencio, P. P.; Samara, G. A., Synthesis and optical properties of colloidal germanium nanocrystals. Physical Review B 2001, 64 (3), 035417.

18_Warner, J. H.; Tilley, R. D., Synthesis of water-soluble photoluminescent germanium nanocrystals. Nanotechnology 2006, 17 (15), 3745. 
19_Niquet, Y. M.; Allan, G.; Delerue, C.; Lannoo, M., Quantum confinement in germanium nanocrystals. Applied Physics Letters 2000, 77 (8), 1182-1184.

20_Sakka, N. S. a. Y., Controlled organic/inorganic interface leading to the size-tunable luminescence from Si nanoparticles. Journal of the Ceramic Society of Japan 2010, 118 (10), 932-939.

21_Shiohara, A.; Prabakar, S.; Faramus, A.; Hsu, C.-Y.; Lai, P.-S.; Northcote, P. T.; Tilley, R. D., Sized controlled synthesis, purification, and cell studies with silicon quantum dots. Nanoscale 2011, 3 (8), 3364-3370.

22_Wheeler, L. M.; Levij, L. M.; Kortshagen, U. R., Tunable Band Gap Emission and Surface Passivation of Germanium Nanocrystals Synthesized in the Gas Phase. The Journal of Physical Chemistry Letters 2013, 3392-3396.

23_Eaton, D., (1988). "Reference materials for fluorescence measurement." Pure \& Applied Chemistry 60(7): 1107-1114

24_Kirby, E. P.; Steiner, R. F., Influence of solvent and temperature upon the fluorescence of indole derivatives. The Journal of Physical Chemistry 1970, 74 (26), 44804490.

25_Leaver, I. H.; Lennox, F. G., Studies of the photodegradation of tryptophan. Photochemistry and Photobiology 1965, 4 (3), 491-497.

26_Resch-Genger, U.; Grabolle, M.; Cavaliere-Jaricot, S.; Nitschke, R.; Nann, T., Quantum dots versus organic dyes as fluorescent labels. Nat Meth 2008, 5 (9), 763-775.

27_Mancini, M. C.; Kairdolf, B. A.; Smith, A. M.; Nie, S., Oxidative Quenching and Degradation of Polymer-Encapsulated Quantum Dots: New Insights into the Long-Term Fate and Toxicity of Nanocrystals in Vivo. Journal of the American Chemical Society 2008, 130 (33), 10836-10837. 
28_Ghosh, B.; Sakka, Y.; Shirahata, N., Efficient green-luminescent germanium nanocrystals. Journal of Materials Chemistry A 2013, 1 (11), 3747-3751.

29_Prabakar, S.; Shiohara, A.; Hanada, S.; Fujioka, K.; Yamamoto, K.; Tilley, R. D., Size Controlled Synthesis of Germanium Nanocrystals by Hydride Reducing Agents and Their Biological Applications. Chemistry of Materials 2009, 22 (2), 482-486.

30_ Vaughn Ii, D. D.; Schaak, R. E., Synthesis, properties and applications of colloidal germanium and germanium-based nanomaterials. Chemical Society Reviews 2013, 42 (7), 2861-2879.

31_Huang, S.; So, Y. H.; Conibeer, G.; Green, M. A., In situ formation of tin nanocrystals embedded in silicon nitride matrix. Journal of Applied Physics 2009, 105 (12), 124303-5.

32_Li, Z.; Tao, X.; Cheng, Y.; Wu, Z.; Zhang, Z.; Dang, H., A facile way for preparing tin nanoparticles from bulk tin via ultrasound dispersion. Ultrasonics Sonochemistry 2007, $14(1), 89-92$.

33_Lai, S. L.; Guo, J. Y.; Petrova, V.; Ramanath, G.; Allen, L. H., Size-Dependent Melting Properties of Small Tin Particles: Nanocalorimetric Measurements. Physical Review Letters 1996, $77(1), 99$.

34_Zou, C.-d.; Gao, Y.-I.; Yang, B.; Zhai, Q.-j., Size-dependent melting properties of Sn nanoparticles by chemical reduction synthesis. Transactions of Nonferrous Metals Society of China 2010, 20 (2), 248-253.

35_Coppinger, M.; Hart, J.; Bhargava, N.; Kim, S.; Kolodzey, J., Photoconductivity of germanium tin alloys grown by molecular beam epitaxy. Applied Physics Letters 2013, $102(14),-$. 
36_Asom, M. T.; Kortan, A. R.; Kimerling, L. C.; Farrow, R. C., Structure and stability of metastable alpha-Sn. Applied Physics Letters 1989, 55 (14), 1439-1441.

37_ Yun Hwan, J.; Inyu, J.; Chung Seok, C.; Inyoung, K.; Hyuck Mo, L., Synthesis and characterization of low temperature $\mathrm{Sn}$ nanoparticles for the fabrication of highly conductive ink. Nanotechnology 2011, 22 (22), 225701.

38_Cui, Q.; Rajathurai, K.; Jia, W.; Li, X.; Gao, F.; Lei, Y.; Gu, Z., Synthesis of Single Crystalline Tin Nanorods and Their Application as Nanosoldering Materials. The Journal of Physical Chemistry C 2010, 114 (50), 21938-21942.

39_Groves, S.; Paul, W., Band Structure of Gray Tin. Physical Review Letters 1963, 11 (5), 194.

40_ King, H. W., Quantitative size-factors for metallic solid solutions. Journal of Materials Science 1966, 1 (1), 79-90.

41_Pukite, P. R.; Harwit, A.; Iyer, S. S., Molecular beam epitaxy of metastable, diamond structure $\mathrm{Sn}_{\mathrm{x}} \mathrm{Ge}_{1-\mathrm{x}}$ alloys. Applied Physics Letters 1989, 54 (21), 2142-2144.

42_Olesinski, R. W.; Abbaschian, G. J., The Ge-Sn (Germanium-Tin) system. Bulletin of Alloy Phase Diagrams 1984, 5 (3), 265-271

43_Wilcoxon, J. P.; Provencio, P. P.; Samara, G. A., Synthesis and optical properties of colloidal germanium nanocrystals. Physical Review B 2001, 64 (3), 035417. 



\section{Silicon quantum dots: towards their use in solar cells}

The optical properties of silicon quantum dots give rise to possible applications for optoelectronic devices, such as solar cells. Quantum dot-sensitized solar cells have developed a great interest due to the advantages of quantum dots compared to organic dyes, such as an increased fluorescence intensity and a greater resistance to photobleaching. ${ }^{1-3}$ Progress with quantum dots devices so far has involved mostly group II-VI or group IV-VI materials. The first quantum dots sensitized solar cell (QD-SSC) was reported by Nozik in 2002, using InP quantum dots associated to $\mathrm{TiO}_{2}$ nanoparticles. ${ }^{4}$ Kamat reported a similar structure with CdSe QDs in $2006 .{ }^{5}$ The highest efficiency for CdS/CdSe QD-SSC reaches $4.8 \% .^{6}$ Quantum dots photovoltaics offer a lot of prospects towards cheap and easily processable solar cells, ${ }^{3}$ but the toxicity of cadmium and the diminishing resources of indium encourages research towards other material quantum dots. $^{7}$

Due to its abundance and low toxicity, silicon quantum dots offer a significant alternative. In this research, it was first attempted to create a composite of silicon quantum dots with zinc oxide nanowires. This research was instigated by previous work with dye-sensitized solar cells using $\mathrm{ZnO}$ nanowires ${ }^{8-10}$ and quantum dots-sensitized solar cells (usually lead or cadmium-based quantum dots) anchored to titanium dioxide or zinc oxide films. ${ }^{4,11-12}$ The effect of silicon quantum dots will be measured by comparing the optical absorption and emission of the composite in comparison that of bare $\mathrm{ZnO}$ nanowires. This research was conducted with the help of my co-supervisor Dr. Natalie Plank. The Watkins' group reported the use of silicon quantum dots to force the separation of block copolymers in multiple morphologies. ${ }^{13}$ The optical properties of the silicon quantum dots immersed in a block co polymer matrix were investigated. Last, the function of silicon quantum dots laid onto a silicon solar cell was investigated. This research was conducted in partnership with Prof. Maan Alkaisi (University of 
Canterbury, NZ). The role of silicon nanocrystals was appraised by measuring the current density-voltage evolution compared to a plain solar cell.

In this chapter will be first described the growth of zinc oxide nanowires and the attachment of silicon quantum dots to the nanowires array. The $\mathrm{ZnO}$ nanowires were characterized using SEM and TEM. The optical properties of the composite silicon QDs/ZnO nanowires were assessed using UV-Visible and photoluminescence spectroscopies. The silicon quantum dots blended in block copolymers were also characterized using UV-Visible and photoluminescence spectroscopies. Finally the role of silicon nanocrystals on the electrical properties of a doped silicon solar cell will be characterized by measuring the voltage generated by the cell upon variation of the current density.

\subsection{Introduction to solar cells}

A basic solar cell is typically composed of one photoactive layer, an anode and cathode. Electrons in the photoactive layer are excited by the energy of the absorbed photons. Through a careful engineering of the energy bands of the electrode material, the excited electrons will transfer to the anode instead of relaxing to their ground state, thus creating an electron flow, and consequently an electric current. Two main types of solar cell architecture will be discussed in this chapter. The silicon solar is composed of silicon doped with negative charge carriers (n-type) or positive charge carriers ( $p$-type). The electrons are generated in the p-type layer (fig. 6.1.a) and flow through the n-type silicon. In the dye-sensitized or quantum dots-sensitized solar cell, the electron acceptor is placed between the incoming light and the photoactive layer, either dye or quantum dots (fig. 6.1.b). In that case, the electrode is composed of a transparent conductive oxide layer, such as zinc oxide. 


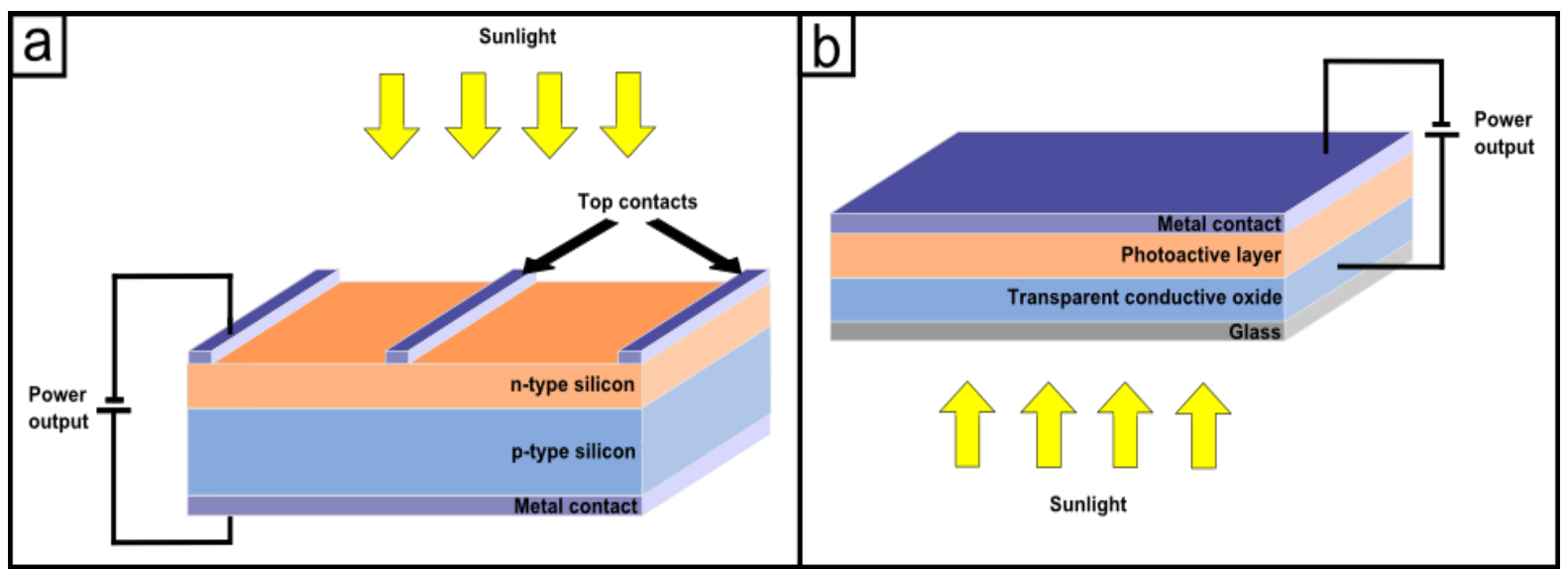

Figure 6.1: Schematics of a) a silicon solar cell and b) dye-sensitized or quantum dotssensitized solar cell

Starting from these basic structures, the efficiency of a solar cell can be increased following two different approaches. The first approach is the multi-junction (M-J) solar cell, in which multiple single junction cells are assembled. The layers are stacked on top of each other to facilitate the electron transfer between the different layers, through a bandgap cascade. The losses are limited due to the decrease of electron-hole recombination processes, thus increasing the efficiency. Also, multi-junction solar cells benefit from a discrepancy in absorbed wavelength to enlarge the domain of absorbed wavelength from the solar spectrum. Multiple junction solar cells have the highest theoretical efficiency ${ }^{14}$ and the highest recorded efficiency conversion (44.4\%) has been obtained from an M-J cell by Sharp Corporation in 2013 under concentrated solar illumination. ${ }^{15}$ Most reported materials for $\mathrm{M}$-J cells are III-V semiconductors, such as InP or GaAs. ${ }^{16-18}$

The efficiency of a dye-sensitized or quantum dot-sensitized solar cell can be enhanced by the architecture of the junction between the photoactive layer (donor) and the anode (acceptor). ${ }^{19,20}$ For an effective electron transfer, the distance between the newly excited electron and the anode material should not exceed the exciton diffusion length (typically between 5 and $20 \mathrm{~nm}$ ), otherwise it will recombine. Figure 6.2 represents four different interface morphologies that can be used in a solar cell, adapted from organic photovoltaic device architectures. 


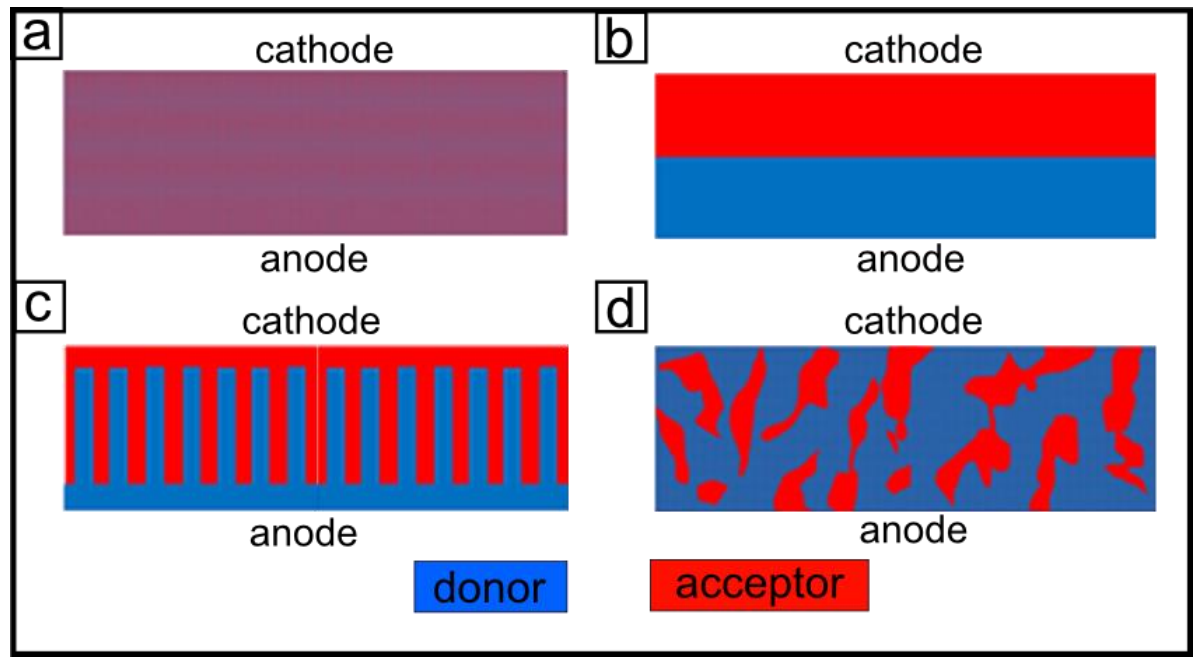

Figure 6.2: Different designs of surface interaction morphologies in solar cells: a)

blended, b) planar heterojunction, c) nanowires and d) typical morphology in organic solar cells (adapted from Sariciftci's article) ${ }^{20}$

In a fine dispersion or blended morphology (fig. 6.2.a) the distance between electron donor and acceptor is ideal, but the charge transport is poor. The bilayer morphology in figure 4.2.b) has the opposite characteristics: the charge transport is favourable, but charge generation would be limited to the interface. Ideal performance has been simulated and achieved through the nanowire architecture (fig. 6.2.c) ${ }^{21-23}$, owing to an increased area and continuous donor-acceptor interface and an efficient charge separation if the distance between wires does not exceed twice the diffusion length of an exciton. ${ }^{24}$ Figure 6.2.d) represents the typical morphology encountered in bulk heterojunction solar cells or organic cells.

The effectiveness of a solar cell can be described by several characteristic parameters. Most of those can be extracted from current-voltage plots, which feature the decreasing current density with increasing voltage obtained by a variation of the resistance. 


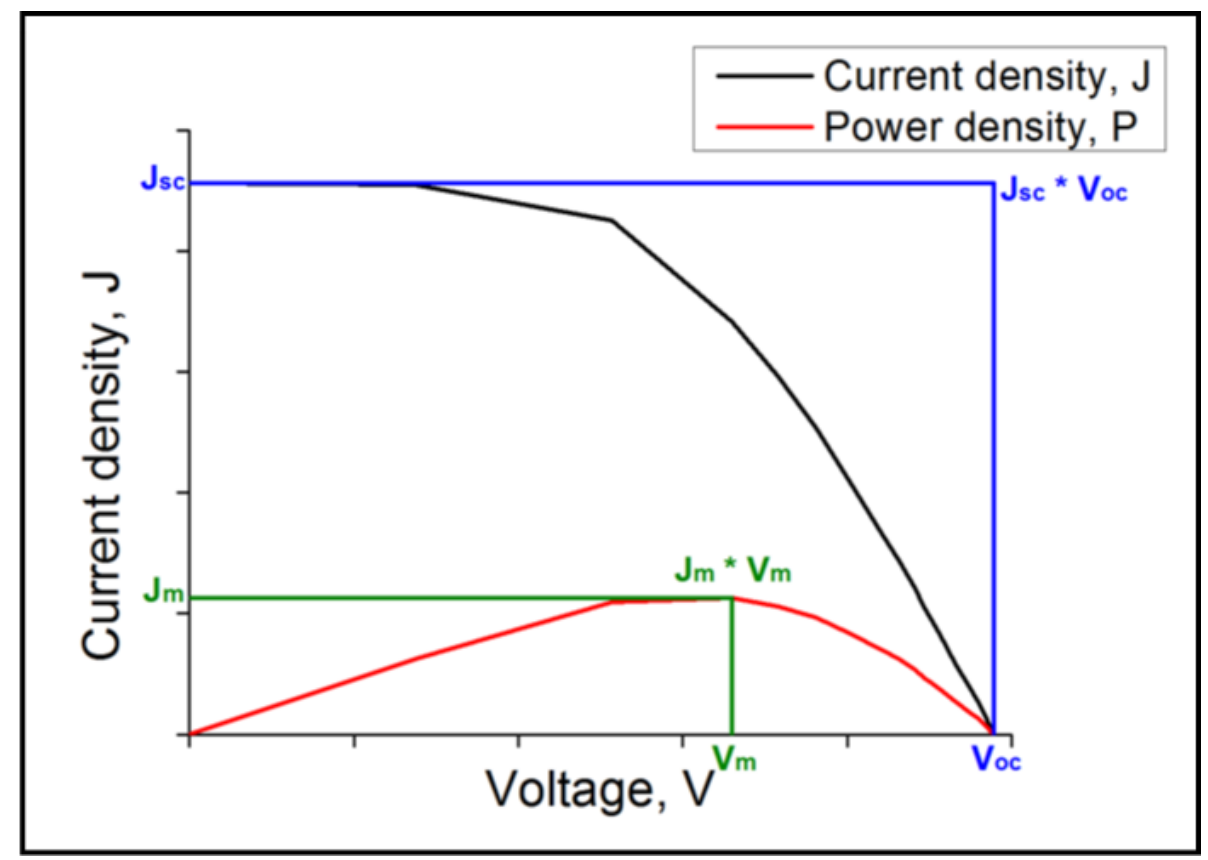

Figure 6.3: Typical J-V characteristic curve of a solar cell

The fill factor $(F F)$ is one common parameter used to characterise solar cells. It is defined as the ratio of the maximum efficiency of the cell divided by the theoretical maximum power, obtained by multiplying the short circuit current density $J_{s c}$ (or $\mathrm{J}$ at $\mathrm{V}=0$ ) and the open circuit voltage $V_{o c}$ (or $V$ at $\mathrm{J}=0$ ).

$$
F \bar{F}=\frac{J_{m} V_{m}}{J_{s c} V_{o c}}
$$

The maximum efficiency or power conversion efficiency $\eta$ is defined as the ratio of the maximum power output of the solar cell to the power of the incoming light (PS).

$$
\eta=\frac{J_{m} V_{m}}{P_{s}}=\frac{J_{s c} V_{o c} F^{3} F^{3}}{P_{s}}
$$

$J_{s c}, V_{o c}, F F$ and $\eta$ are the quantities the most frequently used to characterize solar cells, generally under the standard conditions of a temperature of $25^{\circ} \mathrm{C}$ and a light input power of 1 sun or $1000 \mathrm{~W} / \mathrm{m}^{2}$.

One other characteristic plot for efficiency is found in the literature: the Incident Photon Conversion Efficiency (IPCE) plot. It represents the wavelength dependence of the quantum efficiency, which is the ratio between the number of charge carriers collected by the solar cell and the number of incident photons. 


\subsection{Silicon quantum dots solar cells: an overview}

Today's photovoltaic market is largely dominated by silicon solar cells, but yet silicon quantum dots are not commonly found in solar cells. The localization of electrons in silicon quantum dots offers promising prospects for third generation of solar cells. ${ }^{25}$

Silicon quantum dots are first used in hot carriers solar cells, in which silicon quantum dots are used to reduce the energy losses through thermalization of photons with an energy much greater than the bandgap of the active photovoltaic material. Nozik and co-workers suggested the possibility that high energy photons can be split into two or more lower energy photons. ${ }^{26}$ This theory is called multiple exciton generation. Gregorkiewicz and co-workers later demonstrated the photon cutting effect. ${ }^{27} \mathrm{~A}$ single photon produces two electron-holes pairs that recombine to produce a single highly energetic exciton. This principle has great potential to improve the solar cell efficiency while reducing the losses through heat. It has been reported for direct bandgap materials, such as PbSe. ${ }^{28}$ Recently, Uchida et al reported the photocurrent generation in silicon QDSSC for quantum dots deposited via plasma assisted chemical vapour deposition. ${ }^{29}$

Silicon quantum dots can also be used to enhance the performances of all silicon tandem-multi junction solar cells. Bands of silicon quantum dots in a silicon dielectric matrix $\left(\mathrm{SiO}_{2}\right.$ or $\left.\mathrm{Si}_{3} \mathrm{~N}_{4}\right)$ are formed by annealing silicon rich co-sputtered layers. ${ }^{30}$ These bands create wide bandgap $p-n$ junctions that generate an increased efficiency ${ }^{31}$

Last, silicon quantum dots have developed some interest for Inorganic-organic solar cells. Kortshagen reported a hybrid solar cell using SiQDs and P3HT showing an increase of both open-circuit voltage and short-circuit current and a maximum efficiency of $1.15 \% .^{32}$

Previous work by Kim et al reports that using silicon quantum dots as an intermediate layer between a film of $\mathrm{TiO}_{2}$ nanoparticles and N719, a commercial dye for DSSCs, can increase the efficiency of the cell from $5.2 \%$ to $6.2 \%{ }^{33}$ 


\subsection{Silicon quantum dots anchored to zinc oxide nanowires}

Silicon quantum dots are expected to be light harvesters or "hot carriers" absorbers due to its large bandgap when quantum confined, and thus electron donors, when included in a photovoltaic device. The nanowire architecture increases the surface area between the electron donor (dye or QDs) and acceptor $\left(\mathrm{ZnO}\right.$ or $\left.\mathrm{TiO}_{2}\right)$ and limits the charge recombination process. ${ }^{34,35}$ In this research, it is proposed to use zinc oxide nanowires to anchor functionalized silicon quantum dots, and to monitor the influence of the quantum dots on the optical properties of the composite.

\subsubsection{ZnO nanowires growth and characterization}

$\mathrm{ZnO}$ is a non-toxic n-type semiconductor that has favourable energy bands for forming heterojunction solar cells with a large $3.2 \mathrm{eV}$ band gap. It can be synthesized via a hydrothermal process, suitable for flexible substrates. ${ }^{36,37}$ We chose that synthetic protocol as it does not require elevated temperature and therefore it is suitable for most substrates. This method was also chosen by reason of the expertise of Dr. Natalie Plank, by whom I was guided closely on this project.

\section{Experimental}

To initialize the growth of the nanowires, a seed layer is needed. A seed solution of zinc acetate $0.005 \mathrm{M}$ in water can be drop casted or spin coated onto a substrate. For a more uniform seed layer, a thin film of $\mathrm{ZnO}$ is sputtered on the substrate using a RF-sputter coater with a $\mathrm{ZnO}$ target. The substrate coated by the seed layer is then placed in an aqueous solution containing $0.02 \mathrm{M}$ of hexamethylenetetramine (HMT) and $0.02 \mathrm{M}$ hydrated zinc nitrate. The solution is kept at around $90^{\circ} \mathrm{C}$ for a few hours. After growth, the nanowires are simply rinsed in deionised water and dried on a hot plate heated at $100^{\circ} \mathrm{C}$.

X-ray diffraction is used to determine the crystal structure of the nanowires, which can then be compared to a standard powder diffraction pattern for zinc oxide. By observing the extinction of some crystallographic planes reflections, we can identify the orientation of the $\mathrm{ZnO}$ nanowires. The XRD analysis of the $\mathrm{ZnO}$ nanowires grown on a silicon wafer is presented in figure 6.4. 


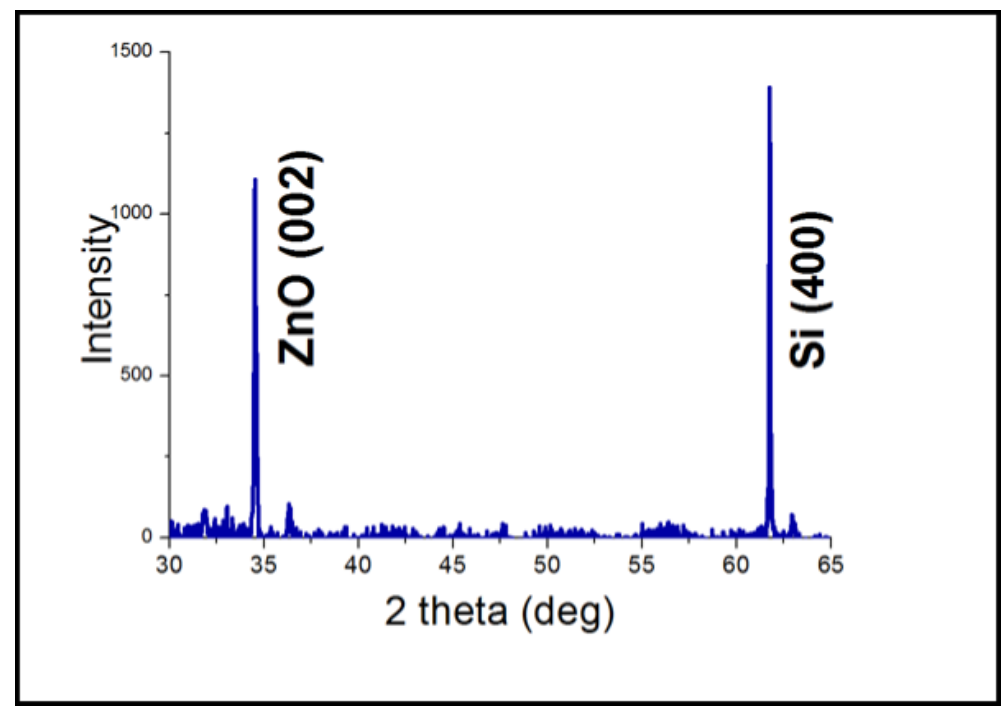

Figure 6.4: XRD spectrum for $\mathrm{ZnO}$ nanowires grown onto a commercially available silicon wafer.

The spectrum presented in figure 6.4 presents only two peaks. By comparison with the reflections to the ones reported in the standard powder diffraction for zinc oxide materials (JCPDS card 36-1451). The first reflection corresponds to the (002) orientation of zinc oxide. The second peak is attributed to the (400) orientation of the silicon wafer. As the zinc oxide peaks correspond to the reflections (100), (101), (102), (110) and (103) expected in the range $30-65^{\circ}$ are not present for our sample, it can be deduced that the zinc oxide is oriented along the c-axis only, which is consistent with a nanowire arrangement grown perpendicular to the surface of the substrate.

Figure 6.5 shows two SEM images obtained for the zinc oxide nanowires grown onto a silicon wafer. The top view shows the tips of the zinc oxide nanowires and the cross section image manifests the $c$-axis orientated growth. From the cross-section image, we measure that a three hours hydrothermal growth produces nanowires averaging $600 \mathrm{~nm}$ in length. 


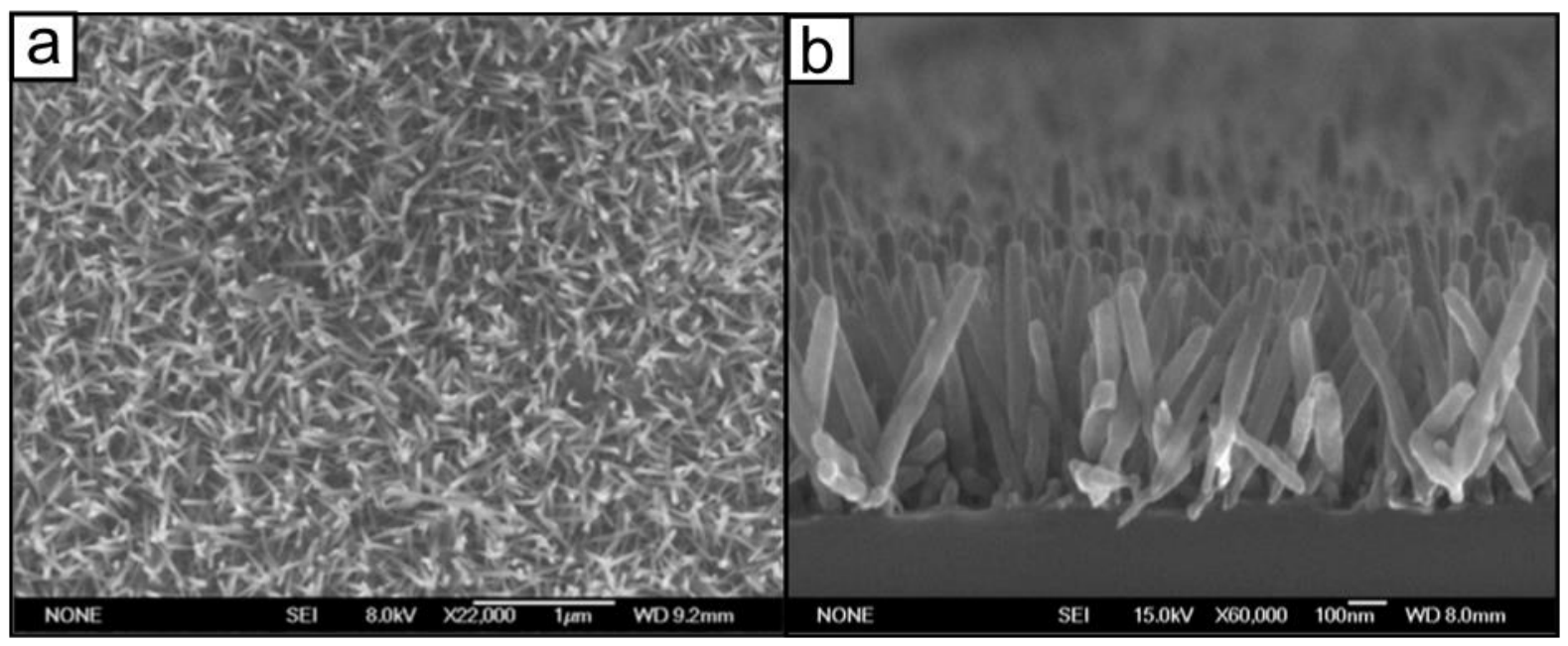

Figure 6.5: SEM images of ZnO nanowires grown onto a piece of silicon wafer. a) top view of the nanowires and $b$ ) their view in cross section.

To prepare the sample for TEM, the nanowires were shaved off the silicon wafer using a razor blade and dispersed in ethanol. The solution was then drop cast onto a carbon coated copper grid. The low resolution TEM image presented in figure 6.6.a) displays the elongated shape of the nanowires. The high resolution TEM image (figure 6.6.b) represents one set of lattice fringes orientated along the $c$-axis, proving that the nanowires are monocrystalline and single oriented.

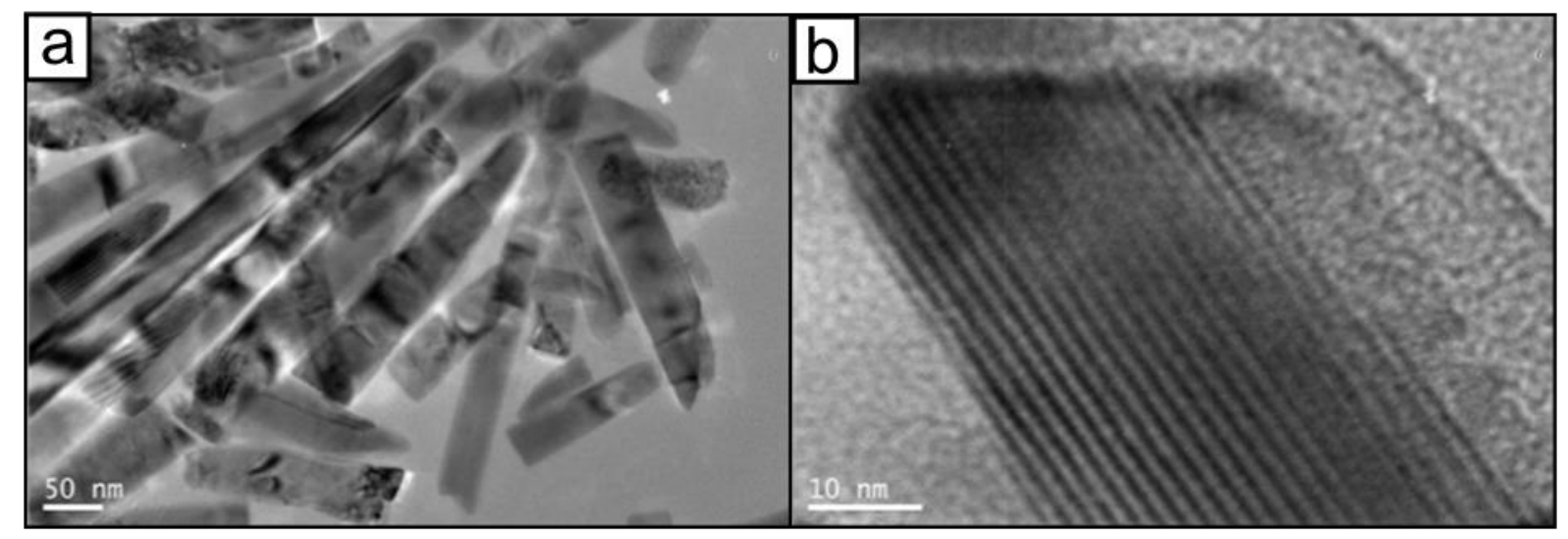

Figure 6.6: TEM images of zinc oxide nanowires. a) is a low resolution TEM image and b) a high resolution TEM image showing the lattice fringes of a single nanowire.

The growth of zinc oxide nanowires was successful by following the method reported by Plank et al. ${ }^{37}$ However, the even production of nanowires over the whole surface of the substrate has revealed itself to be challenging. The most homogeneous samples were 
used to attach silicon quantum dots to the $\mathrm{ZnO}$ nanowires, described in the following section.

\subsubsection{Silicon quantum dots and zinc oxide nanowires composite}

To attach the silicon quantum dots to the surface of zinc oxide nanowires, the substrate with the nanowire array grown onto it was immersed into a silicon nanocrystals solution (cf. figure 6.7). This soaking method was chosen for its practicality as it does not require expensive equipment and its proven efficacy in scientific literature. Similar methods have been reported efficient in the literature for $\mathrm{CdSe}$ or $\mathrm{InP}$ with $\mathrm{TiO}_{2}{ }^{38-40}$ Allylamine terminated silicon quantum dots were chosen for this study as they are soluble in polar solvents, thus easily dispersible in water.

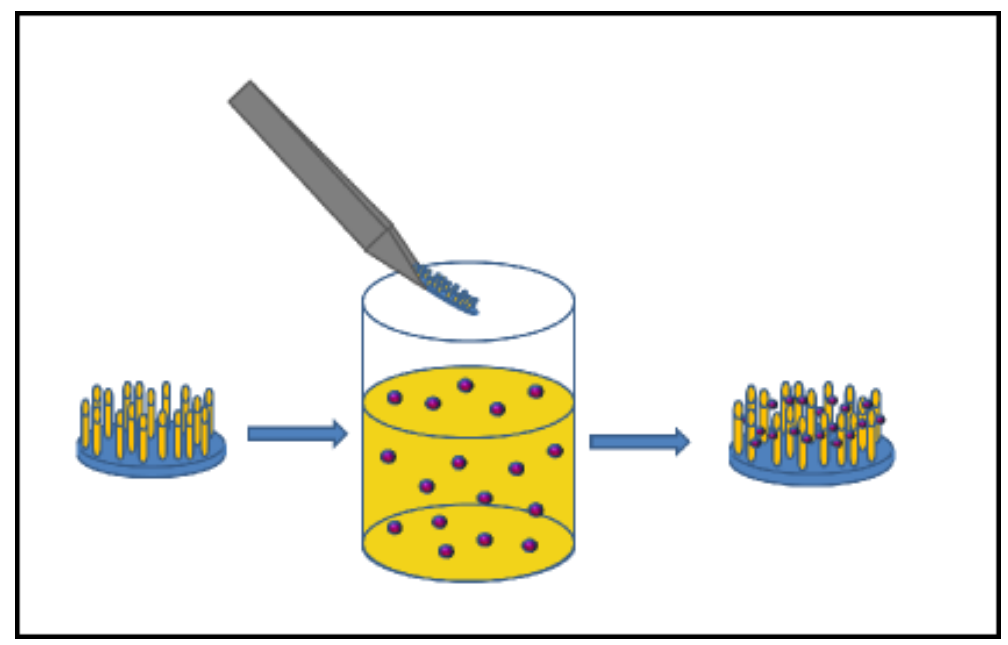

Figure 6.7: Schematic of the process in order to create a uniform composite ZnO nanowires / silicon quantum dots

To observe and measure the optical properties, zinc oxide nanowires are grown onto Spectrosil substrates. Those substrates are made of fused quartz and present an excellent transmission throughout the range from far UV to visible wavelength. The coating of zinc oxide nanowires with silicon quantum dots is expected to show a variation of absorption in the UV range. Figure 6.8 is the absorption spectra of the neat ZnO nanowires and the same $\mathrm{ZnO}$ nanowires array soaked for 6 hours in a silicon quantum dots solution. The dotted line graph is the absorption of the allylamineterminated silicon nanocrystals in solution. 


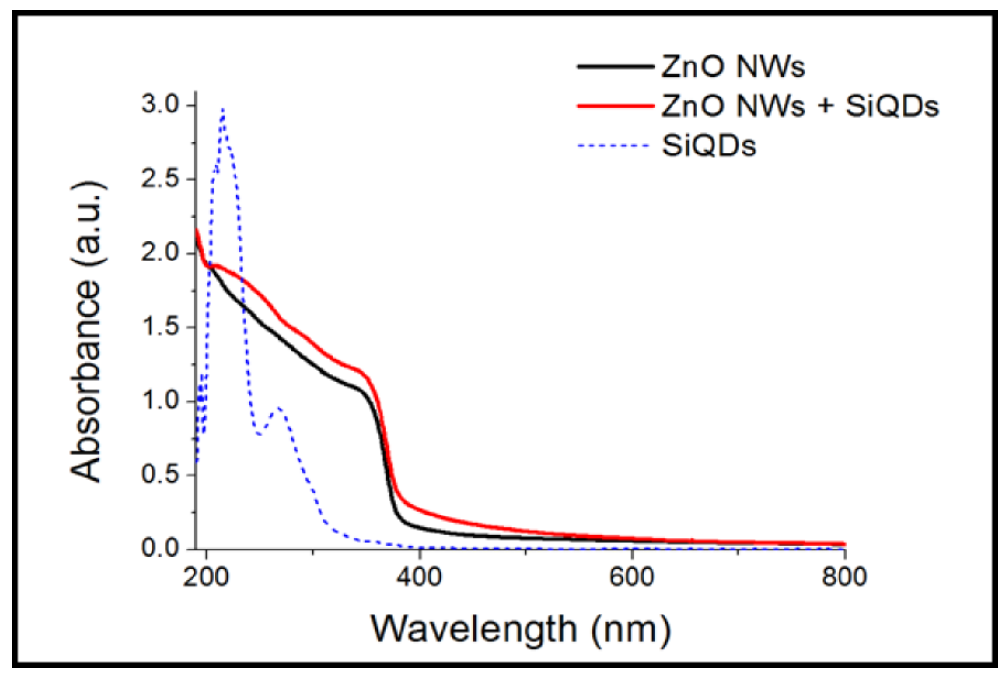

Figure 6.8: UV-Visible spectra of neat ZnO nanowires and ZnO nanowires soaked in a silicon quantum dots solution for 6 hours. The dotted line represents the absorption spectrum of the silicon quantum dots capped with allylamine in solution.

The nanowires array soaked in silicon quantum dots solution shows a slight increase of absorbance over the region between 200 and $270 \mathrm{~nm}$ compared to the bare nanowires. As the silicon quantum dots in solution absorb the same range of wavelength, we can conclude to the increase is due to the attachment of silicon quantum dots to the surface of $\mathrm{ZnO}$ nanowires.

The fluorescence of zinc oxide nanowires is expected to be located at longer wavelength than the fluorescence of silicon quantum dots, as the band gap is higher for silicon nanocrystals than zinc oxide ( 3.4 to $3.8 \mathrm{vs} .3 .2 \mathrm{eV}$ ). ${ }^{41,42}$ It is anticipated that there will be a variation of the fluorescence intensity with increasing silicon quantum dots concentration, achieved through a longer soaking time. The photoluminescence of the ZnO nanowires before and after soaking in the silicon quantum dots solution is represented in figure 6.9. All spectra were recorded at the excitation wavelength $\lambda_{\text {exc }}=$ $320 \mathrm{~nm}$. 


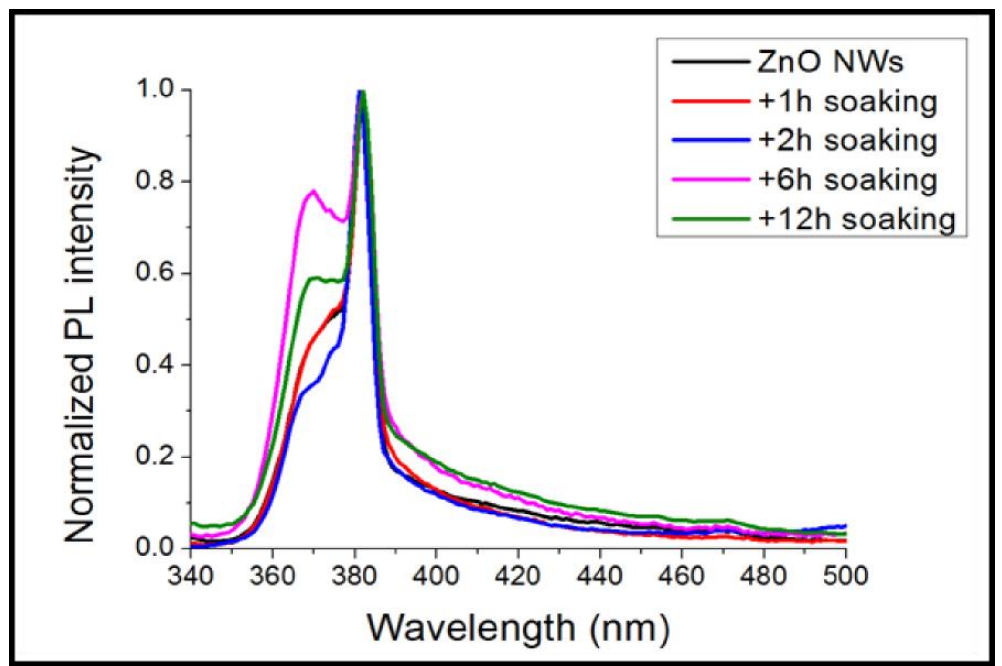

Figure 6.9: Normalized photoluminescence intensity after soaking of the $\mathrm{ZnO}$ nanowires in a silicon quantum dots solution.

The sharp peak at $\lambda_{\mathrm{em}}=383 \mathrm{~nm}$ is assigned to the $\mathrm{ZnO}$ nanowires, as the band gap of 3.2 $\mathrm{eV}$ correspond to an emission of $387 \mathrm{~nm}$. This peak is normalized to 1 for all the samples to visualize the evolution of the fluorescence peak of silicon nanocrystals at $\lambda_{\mathrm{em}}=368$ $\mathrm{nm}$. The variation in intensity of the silicon quantum dots fluorescence shows an increasing concentration of silicon quantum dots up to 6 hours of the soaking time. It correspond to an increase of about $60 \%$ of the PL intensity at $368 \mathrm{~nm}$ compared to the neat ZnO nanowires. This intensity reduces after 12 hours in a bath of silicon nanoparticles, and corresponds only to a $20 \%$ increase of the emission at $368 \mathrm{~nm}$. The variation of intensity of the PL intensity upon soaking time shows the increasing concentration of silicon quantum dots, indicating a successful anchorage of silicon nanocrystals to the surface of the $\mathrm{ZnO}$ nanowires. The decrease of intensity after 12 hours can be explained by a partial dissolution of the zinc oxide nanowires in the quantum dots solution, which is slightly acidic. The $\mathrm{pH}$ of the solution was estimated around 6 with $\mathrm{pH}$ paper.

The even growth of zinc oxide nanowires reveals itself to be challenging. The soaking method for the attachment of silicon quantum dots is successful up to 6 hours, but can be difficult to reproduce as a variation of concentration of the soaking solution can result in a decrease in $\mathrm{pH}$. In addition, $\mathrm{ZnO}$ is an amphoteric oxide: it is nearly insoluble 
in water, but can be degraded by both acidic and basic solutions. ${ }^{43,44}$ This deterioration phenomenon has also been observed for dye sensitized solar cells using $\mathrm{ZnO}$ and the commercially available dye N719 with prolonged adsorption times. ${ }^{45} \mathrm{~A}$ careful design of the surface chemistry of both the ZnO NWs and the silicon quantum dots could decrease the degradation problem and increase the attachment of the silicon quantum dots through hydrogen bonding. ${ }^{25}$

\subsection{Silicon quantum dots in block co-polymers}

Organic or bulk heterojunction (BHJ) solar cells have attracted a lot of interest as cost effective photovoltaics. ${ }^{46}$ The efficiency of organic cells remains generally lower than inorganic cells. BHJ solar cells are usually composed of a donor-type semiconducting polymer (e.g. P3HT) and an electron acceptor-type polymer or molecule such as fullerenes. ${ }^{47}$ The highest recorded efficiency of $12 \%$ for an organic photovoltaic device (OPV) was achieved by the company Heliatek in Germany. ${ }^{48}$ To improve the efficiency, the hybrid organic-inorganic system has developed an increased interest. ${ }^{49,50}$

Block copolymers (BCP) are a good alternative to structured inorganic solar cells combining the ease of processing of the polymers to a microphase separation. ${ }^{51}$ As described earlier, 3D arrangement increases the charge separation and transport. Verduzco et al reported a BCP photovoltaic with near $3 \%$ efficiency. ${ }^{52}$ Block copolymers are a combination of two amphiphillic polymers. The affinity to different environments cause the two polymers to separate and self-organize at the interface, when heated above their glass transition temperature. ${ }^{53}$ This section investigates the optical properties of the silicon quantum dots when blended in a block co-polymer. This research was conducted with the collaboration of the Watkins' group at Massachussets University in Amherst, USA. One of their research projects focuses on block copolymers and their different geometries. They used silicon quantum dots to enhance the separation of the two polymers. ${ }^{13}$ The copolymer chosen for this study was polystyrenepolyethylene oxide (PS-PEO), previously studied by the Watkins group and it has the ability to easily form solid films on the surface of a substrate. The optical absorption and 
emission of the composite was measured for samples prepared using $10 \%$ and $15 \%$ in mass of silicon quantum dots and compared to the neat block copolymer.

\section{Experimental}

The neat PS-PEO sample was prepared by mixing $50 \mathrm{mg}$ of the copolymer into $2 \mathrm{~mL}$ of toluene followed by 2 hours of magnetic stirring. The solution was then drop-casted onto a clean Spectrosil substrate heated at $50^{\circ} \mathrm{C}$ on a hot plate. The thin film was then baked at $100^{\circ} \mathrm{C}$ for one hour.

An allylamine-terminated silicon quantum dots solution was prepared using $8.2 \mathrm{mg}$ of silicon nanocrystals in $2 \mathrm{~mL}$ of tetrahydrofuran (THF). After homogenization for 5 minutes using an ultrasonic bath, $0.8 \mathrm{~mL}$ of that solution were added to $25 \mathrm{mg}$ of PS-PEO to form a $10 \%$ quantum dots mixture. $0.7 \mathrm{~mL}$ of THF were added to the solution and stirred for two hours. The $15 \%$ solution was prepared similarly using $1.2 \mathrm{~mL}$ of the silicon QDs solution mixed to $25 \mathrm{mg}$ of PS-PEO and $0.3 \mathrm{~mL}$ of THF added. The solution was then stirred for two hours using magnetic stirring. The thin films were obtained by drop casting the solution onto Spectrosil substrate heated at $50^{\circ} \mathrm{C}$, followed by a baking at $100^{\circ} \mathrm{C}$ for 2 hours.

The experimental conditions are expected to lead to a cylindrical geometry, polystyrene forming cylinders surrounded by polyethylene oxide. ${ }^{13} \mathrm{~A}$ schematic of the architecture of the SiQDs-(PS-PEO) composite is presented in figure 6.10. The amine-terminated silicon quantum dots are hydrophilic, thus are expected to be located in the PEO phase of the block copolymer.

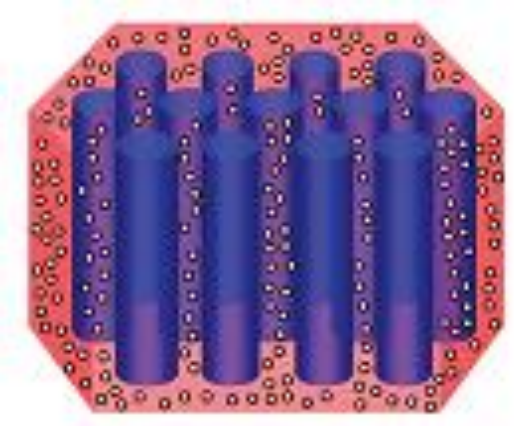

Figure 6.10: Schematic of the PS-PEO block copolymer geometry while blended with hydrophilic nanoparticles under the experimental conditions reported beforehand. ${ }^{13}$ 


\section{Characterization and discussion}

Visually, PS-PEO forms transparent or slightly white films. No difference is distinguishable to the naked eye between the films containing or not containing silicon quantum dots. But as it can be seen in figure 6.11, under UV light, the thin film containing silicon quantum dots exhibit a strong blue luminescence while the neat PSPEO thin film remains transparent.

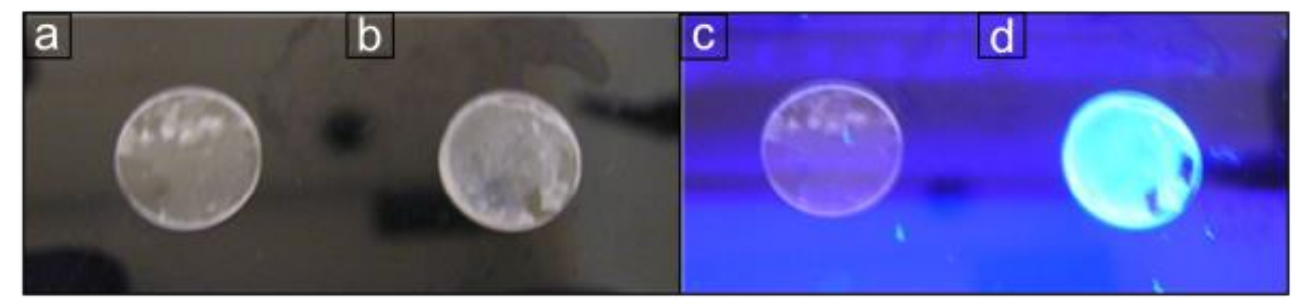

Figure 6.11: Neat PS-PEO thin layer deposited on optically inactive Spectrosil glass under ambient light (a) and UV-light (c). (b) is a picture of a thin layer of PS-PEO blended with $15 \%$ of silicon quantum dots under ambient light and (d) under UV light.

The effect of the silicon quantum dots addition on the absorption of the polystyrenepolyethylene oxide copolymer was fisrt measured. The increasing concentration of silicon quantum dots is expected to produce an increasing absorption response.

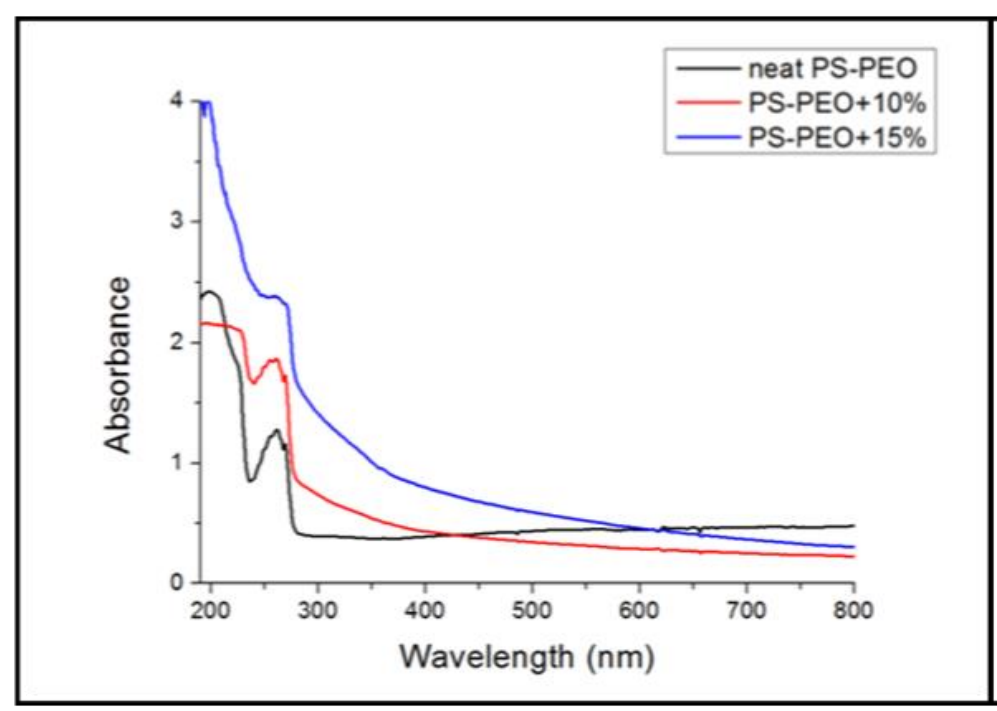

Figure 6.12: Absorbance spectra for neat PS-PEO and PS-PEO blended with 10 and $15 \%$ of silicon quantum dots deposited on Spectrosil substrates. 
The distinction between the absorption of the neat PS-PEO and the ones from the SiQDS-PS-PEO composite is not evident as both the copolymer and the silicon nanocrystals absorb in the same wavelength region (200 - $270 \mathrm{~nm})$. Moreover, both share the same peak features, with a primary peak around $210 \mathrm{~nm}$ and a secondary peak at $265 \mathrm{~nm}$, as it can be seen in figure 6.12. An increase in the absorbance is noticed with increasing concentration of silicon quantum dots, but the origin of this increase cannot be concluded to be due to the increasing concentration of silicon quantum dots as the thickness of the thin film may vary slightly between the samples. Drop casting is not a reproducible process for producing films with a regular thickness. The evidence of the increased absorption due to silicon quantum dots rely on the absorption edge for wavelength up to $500 \mathrm{~nm}$, observed for the two films containing silicon quantum dots, also observed for silicon quantum dots in solution (ref figure 3.12).

To confirm that the observed blue luminescence comes upon the addition of silicon quantum dots to the PS-PEO copolymer, a photoluminescence study of the neat PS-PEO, the 10 and $15 \%$ of silicon nanocrystals containing films is compared to the photoluminescence of silicon quantum dots in solution (figure 6.13). Excitation wavelengths were chosen according to the absorption wavelength of the same samples (figure 6.12) and spectra were recorded between 240 and $380 \mathrm{~nm}$ with $20 \mathrm{~nm}$ intervals. 


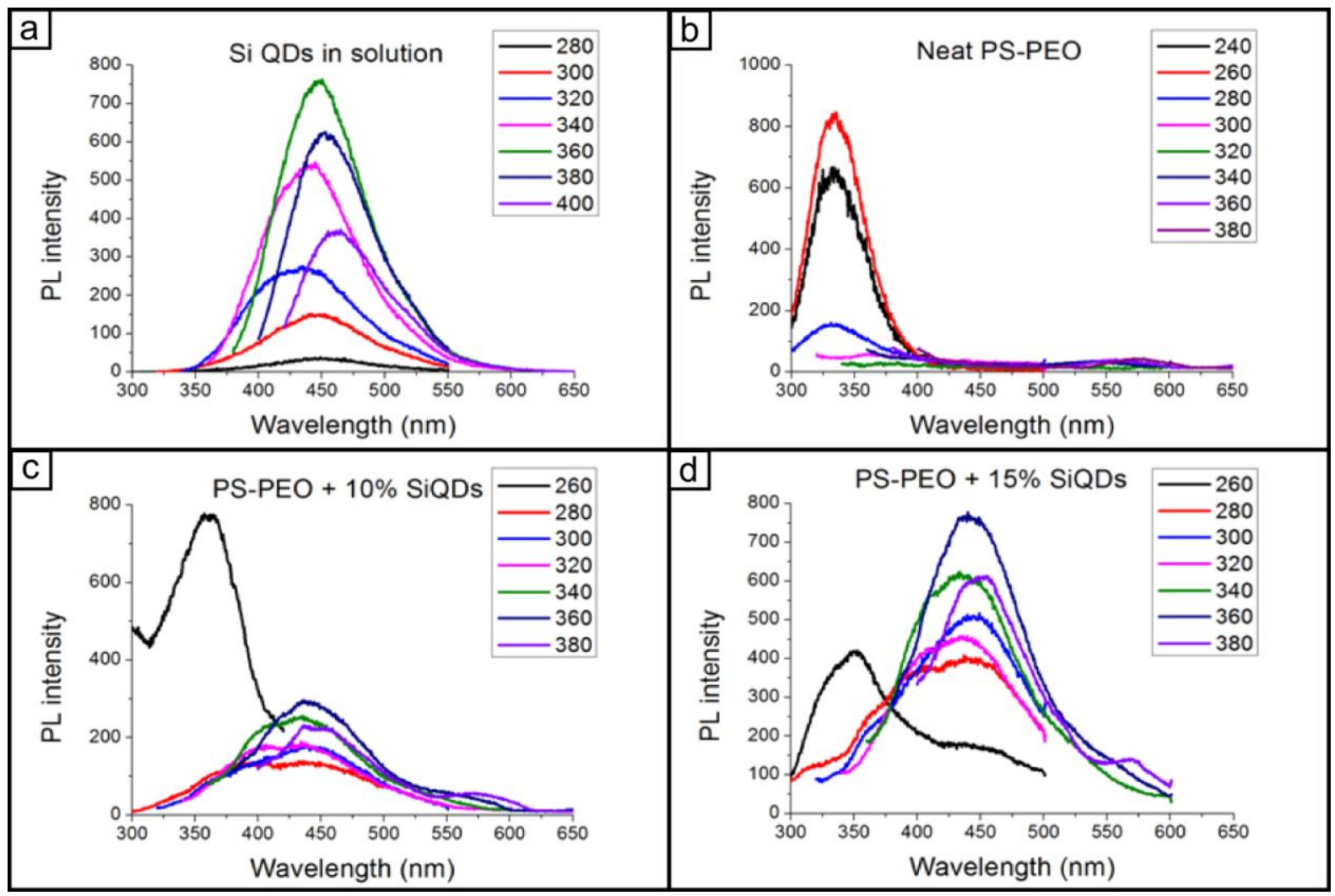

Figure 6.13: Photoluminescence spectra of a) the allylamine-terminated silicon quantum dots solution in methanol, b) the neat copolymer PS-PEO, c) and d) are the PL features of the composite PS-PEO/Si QDs with $10 \%$ and $15 \%$ of silicon quantum dots respectively.

Excited at wavelength between $\lambda$ exc $=300$ and $400 \mathrm{~nm}$, silicon quantum dots exhibit a strong fluorescence around $450 \mathrm{~nm}$ while in solution. The neat PS-PEO film shows a fluorescence emission at $335 \mathrm{~nm}$ when excited with wavelength smaller than $280 \mathrm{~nm}$. It does not show emission in the same range of wavelength than silicon quantum dots. After being blended with silicon nanocrystals, the films exhibit a photoluminescence at the same wavelengths as the silicon quantum dots in solution, with increasing intensity between 10 and $15 \%$ of silicon nanoparticles loading. From these photoluminescence measurements we can conclude that silicon quantum dots contribute to the fluorescence of the composite as the relative intensity increases with increasing concentration of nanocrystals. Also, no shift in emission wavelength is observed between the PL from silicon nanoparticles in methanol solution and the ones combined 
to PS-PEO, evidencing that no obvious electronic interaction is occurring between the quantum dots surface and the block copolymer.

The silicon quantum dots were easily blended into the copolymer by magnetically stirring the composite solution. Silicon quantum dots still present good optical properties loaded in the block copolymer and at identical wavelength as the silicon quantum dots in solution are observed in the photoluminescence study. The cylindrical architecture is similar to the nanowire morphology described in section 6.3 for zinc oxide nanowires. This nano-arrangement makes the PS-PEO block copolymer an alternative to inorganic materials for interface engineering.

These observations are in good agreement with previous work reported in the literature. The Bawendi group found that the emission almost exclusively characteristic of the guest nanocrystals (CdSe or CdSE-ZnS core-shell) with the same 15\% quantum dots concentration. ${ }^{54}$ Similarly, Zorn et al reported a hybrid between CdSe-ZnS core shell nanocrystals and tripenylamine-based $\mathrm{BCP}$ and they observed a conservation of the optical properties of the QDs as well as an easy processing for LED applications. ${ }^{55}$

From these results, PS-PEO is a good polymer template for hybrid organic-inorganic architectures. The nanowire-type of morphology is very attractive for solar cell applications. Additionally, polystyrene and poly ethylene oxide are cheap and easily available and PS-PEO is easy to handle and forms easily thin dry films.

\subsection{Silicon quantum dots coated on silicon solar cell}

To enhance the performance of already commercialized silicon solar cells, an interesting approach is to use silicon quantum dots to improve the scattering and light trapping and possibly to reduce the reflection on the surface of a silicon solar cell. ${ }^{56,57}$ This section aims at observing and understanding the role played by silicon quantum dots in a solar cell. This project was conducted in collaboration with Professor Maan Alkaisi from the 
Canterbury University in Christchurch, New Zealand. On a pre-built solar cell, silicon nanocrystals were deposited on the surface and the solar efficiency was measured.

\subsubsection{Description of the solar cell}

The solar cell used in this study was fabricated in the cleanroom of University of Canterbury in Christchurch, New Zealand by Senthuran Sivasubramaniam. The fabrication of the cell was conducted via the spin-on-dopant principle. ${ }^{58}$ Figure 6.14 describe the structure of the doped silicon solar cell. A $10 \mathrm{~cm}$ diameter silicon wafer holds 21 cells.

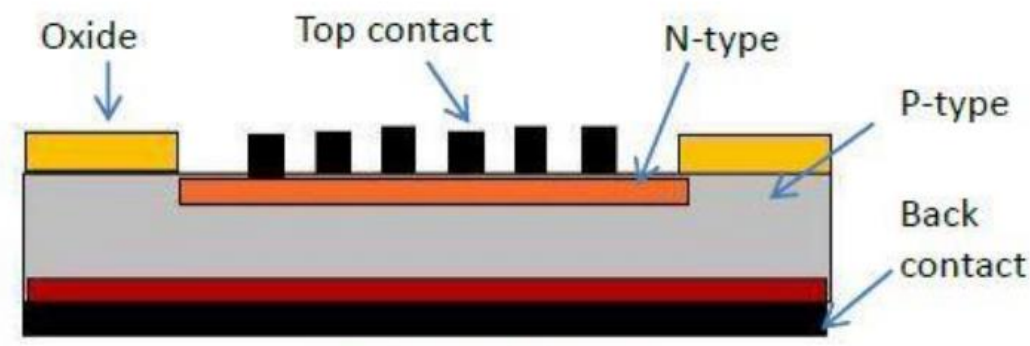

Figure 6.14: Schematic of the spin-on-dopant silicon solar cell fabricated by the Alkaisi group at the University of Canterbury. ${ }^{59}$

\subsubsection{Deposition of silicon quantum dots}

After a thorough cleaning process of the solar cells, the silicon quantum dots solutions were drop casted onto their surface. Silicon quantum dots form a homogeneous film across the surface of the wafer. Three different batches of silicon quantum dots were used in this study. The first batch was synthesized by reduction of silicon tetrachloride using sodium borohydride and further capped with allylamine rendering them water soluble. It is composed of nanocrystals with an average size of $3.7 \pm 2.6 \mathrm{~nm}$ (refer to chapter 3 section 3.3). Later it will be refered to this sample as polydisperse SiQDsamine as the particle range from 1.5 to $7 \mathrm{~nm}$. The second batch was also capped with allylamine, but reduced using lithium borohydride as a reducing agent. The nanocrystals in that specimen measure $2.3 \pm 0.7 \mathrm{~nm}$. As their size distribution is much smaller, we 
refer later to that sample as monodisperse SiQDs-amine. The last solution of silicon quantum dots contains 1-hexene terminated nanocrystals synthesized using lithium borohydride. Their average size is $2.4 \pm 1.0 \mathrm{~nm}$ and we later referenced them as monodisperse SiQDs-1-hexene. The solvent used to disperse the hexane capped particles was chloroform.

The goal of this research is to observe the variation of the photovoltaic response of the silicon solar cell with the added layer of silicon quantum dots and the influence of the size and surface capping of those quantum dots. Silicon quantum dots are expected to increase the overall efficiency of the solar cell. The efficiency variation upon addition of SiQDs will be evaluated by current-voltage plots.

\section{Experimental}

First, the surface of the solar cell was cleaned from any impurity using standard solvent cleaning with acetone then isopropanol followed by oxygen plasma cleaning. The silicon quantum dots solutions were homogenized in an ultrasonic bath for 5 minutes. Allylamine-terminated silicon quantum dots were dispersed in water; the ones capped with 1-hexene were dispersed in chloroform. After cleaning, the solar cells were placed onto a heating plate, heated at $60^{\circ} \mathrm{C}$ for silicon quantum dots dispersed in chloroform or $100^{\circ} \mathrm{C}$ for water solutions. The solutions were then drop casted onto the surface of the solar cell. The solar cells + silicon quantum dots were then placed in an oven at $95^{\circ} \mathrm{C}$ for one hour to remove the possible remaining solvent. The anti-reflective layer is a titanium/silica film type $C$ purchase from Emulstone co. ${ }^{60}$ The anti-reflective film was deposited by spin coating at $3000 \mathrm{rpm}$ for 60 seconds, baked on a hot plate at $200^{\circ} \mathrm{C}$ for 15 minutes before treatment in a tube furnace at $420^{\circ} \mathrm{C}$ for 15 minutes.

After the oven treatment, the contacts were carefully wiped of silicon quantum dots to avoid an overlapping of the cells over the wafer, which could lead to an inaccurate measure of the current density as its value depends on the surface area. 


\subsubsection{Measurement of J-V curves}

Solar cells efficiency is determined from current density versus voltage (or J-V) plots. Those plots record the outgoing voltage in function of the applied current per unit of surface. Several characteristic numbers can be extracted from this plot, such as the open circuit voltage, the short circuit current the fill factor and the maximum efficiency. In this section, we record the J-V characteristics for the untreated solar cell, then for an added layer of one of the three different batches of silicon quantum dots, then with a combination of two layers of silicon quantum dots of different sizes. Finally those characteristics are compared to a solar cell coated with a commercially available antireflective layer. The evaluation of those characteristic numbers will allow to conclude on the role played by the silicon quantum dots coated on the surface of solar cells. The same set of silicon cells was used for each experiment.

The current-voltage characteristics of the solar cells bare or with silicon quantum dots layers were recorded under the light produced by a solar simulator producing a light intensity equivalent to 1 sun $\left(1000 \mathrm{~W} . \mathrm{m}^{-2}\right){ }^{61}$ Figure 6.15 represents the plots observed for the different samples of silicon quantum dots with a schematic of the cross section of the layer added on top of the solar cell. 


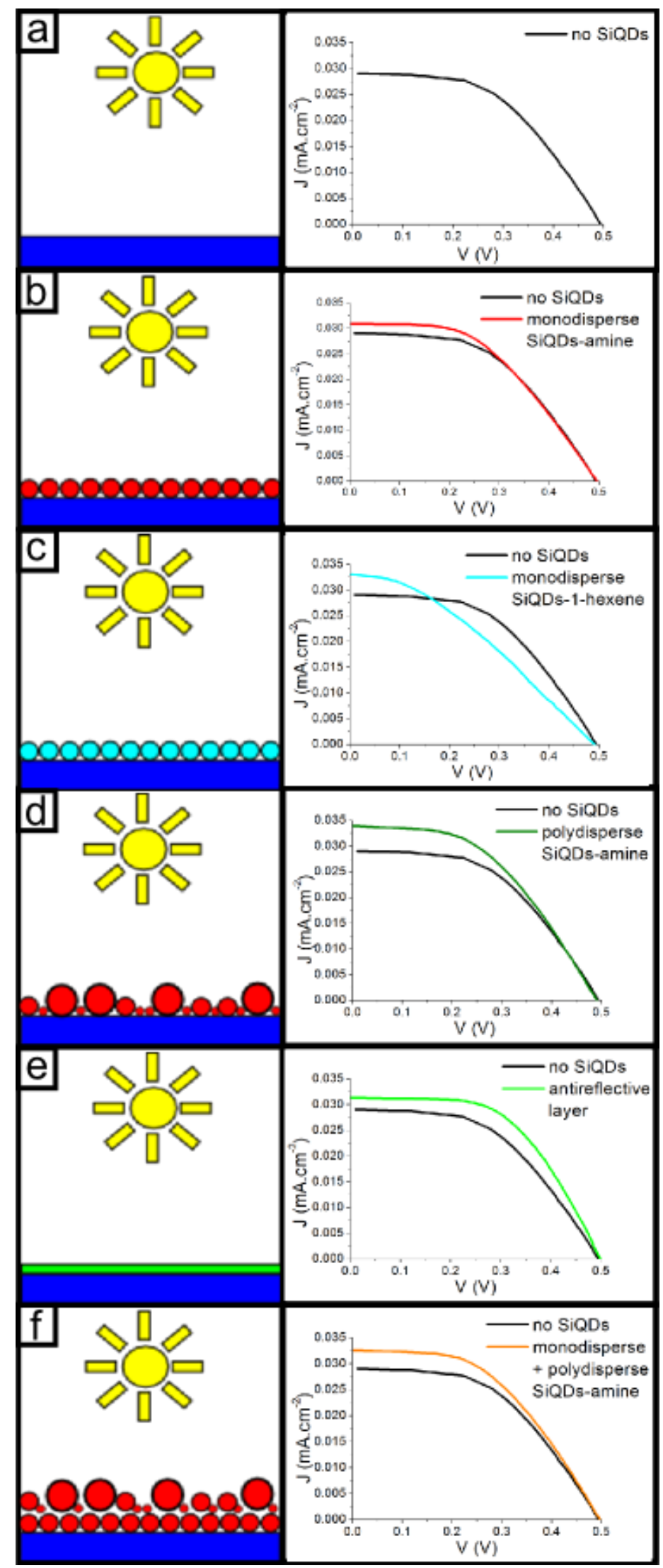

Figure 6.15: Schematic of the different layers deposited onto the spin-on-dopant solar cell and their corresponding J-V curves compared to the plain solar cell under a light exposure of 1 sun. 
Figure 6.15 a) represents the untreated silicon solar cell. The open circuit voltage (at $\mathrm{J}=0$ ) is measured at $0.494 \mathrm{~V}$ and the short circuit current is $29.1 \mathrm{~mA} \cdot \mathrm{cm}^{-2}$. For all different treatments sustained by the cells in this study, the open circuit voltage (Voc) remains constant, while the short circuit current density (Jsc) on increased. Voc is the maximum voltage a solar cell can provide to an external circuit. An increase in Voc would signify a production of extra charges. As Voc remains constant, a reasonable assumption then is that our quantum dots do not generate extra charges when photoexcited or that the produced charges do not travel across the cell and recombine within the dots. Jsc represents the photocurrent without resistance and is proportional to light intensity. As the light intensity was kept constant for all samples, the increase in Jsc for all the coatings is due to an increased photon absorption and a decreased reflection.

On figure $6.15 \mathrm{c}$ ), the solar cell coated with monodisperse SiQDs-1-hexene present a J-V curve that have a different shape than the other J-V curves in this study. The SiQDs-1hexene treated cell present a higher short circuit current than the untreated cell, but from $2.0 \mathrm{~V}$, the SiQDs capped cell present a lower current density than the untreated cell, which indicates a lower maximum power of the cell. A possible explanation for such a difference between this cell and the other SiQDs treated cells is the possible damage of the cell using chloroform as a solvent, as chloroform reacts vigorously with chemically active metals such as aluminium which is used in the contacts.

Considering the general shape of the J-V curves for the amine capped SiQDs coating as well as the antireflective layer coating shown in figure 6.15 b),d),e) and f), it can be assumed an increased performance of the solar cells using silicon quantum dots.

Figure 6.16 synthesizes the I-V curves for all the coatings in comparison to the untreated cell. On this figure is compared the solar cell characteristics of the different coatings used in this research. 


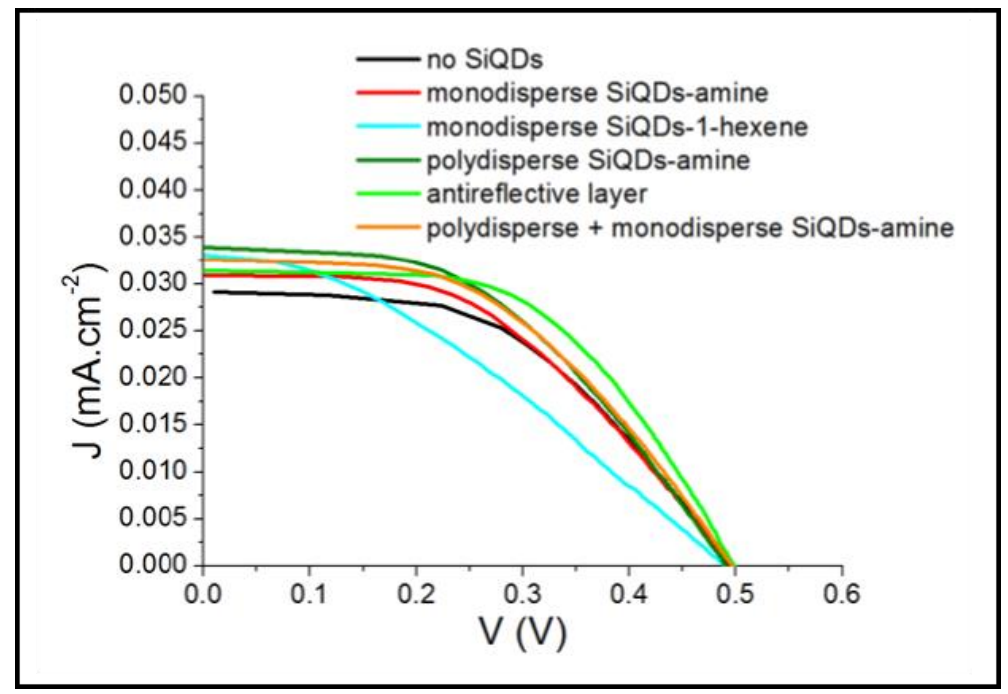

Figure 6.16: J-V curves for the different configuration of the solar cell: neat, with different samples of silicon quantum dots and with an antireflective layer.

The maximum short circuit current is obtained for polydisperse SiQDs-amine coating of the cells. The $\mathrm{NaBH}_{4}$ reduced silicon quantum dots were proven to have a reduced absorption wavelength domain (refer to chapter 3, section 3.3), but here the increase of Jsc can be explained by the texture of the surface. The polydispersity of the nanocrystals forms an irregular surface. An uneven surface is more likely to limit the scattering of incident photons. The cell coated with monodisperse then polydisperse SiQDs show the second highest Jsc, but lower than the cell coated with just polydisperse SiQDs. We can explain this fact by an increase SiQDs layer thickness that could cause an increase in distance for photons to go through before conversion into electrons, creating an increased likelihood of the photons being trapped. The anti-reflective material coated cell exhibits a abrupt decrease in current density for higher voltage value than any other coating. This shape should result in a higher maximum power generated by the cell.

In table 6.1 is presented all the standard characteristic values for solar cells for our samples. Jsc is the short circuit current, Voc the open circuit voltage, defined earlier in this section. The fill factor is the ratio between the theoretical maximum power 
(Jsc*Voc) and the effective maximum power. The efficiency is the ratio between the maximum power and the incident photons energy.

\begin{tabular}{|l|c|c|c|c|}
\hline & Jsc (mA.cm ${ }^{-2}$ ) & Voc (V) & $\begin{array}{c}\text { Fill factor } \\
\text { FF (\%) }\end{array}$ & $\begin{array}{c}\text { Efficiency } \\
\text { П (\%) }\end{array}$ \\
\hline Untreated & 29.1 & 0.494 & 49.6 & 7.14 \\
\hline SiQDs-allylamine polydisperse & 33.9 & 0.491 & 46.8 & 7.78 \\
\hline SiQDs-1-hexene monodisperse & 33.1 & 0.491 & 34.3 & 5.57 \\
\hline SiQDs-allylamine monodisperse & 30.9 & 0.496 & 47.3 & 7.24 \\
\hline Antireflective layer & 31.4 & 0.499 & 54.3 & 8.51 \\
\hline $\begin{array}{l}\text { SiQDs-allylamine polydisperse + } \\
\text { SiQDs-allylamine monodisperse }\end{array}$ & 32.6 & 0.497 & 47.81 & 7.75 \\
\hline
\end{tabular}

Table 6.1: Summary of the characteristics of the solar cell without silicon quantum dots compared to the ones with an added layer of silicon nanocrystals or commercially available anti-reflective layer.

The anti-reflective layer creates a 9,5\% increase from 49.6 to 54.3 on the fill factor compared to the untreated cell. Silicon quantum dots coated cell present all a decrease in the fill factor, even if the Jsc increases. An increase of the maximum efficiency is also observed for the titanium silica film coating, from 7.14 to $8.51 \%$. But an increase in total cell efficiency is observed for all the SiQDs coatings, except the SiQDs-1-hexene, probably due to chloroform damage, as mentioned earlier in this section. Of all the SiQDs coated cells, the highest increase in efficiency is observed for polydisperse SiQDsamine as expected from the J-V plots. The increase in efficiency from 7.14 to 7.78 corresponds to a $9 \%$ increase in efficiency. Although the cell with standard antireflective coating has a greater efficiency increase than any of the Si QD coated cells.

The fill factor represents the power generated by the cell compared to a theoretical maximum value. An increased efficiency shows an enhanced conversion of incident photons into electrons. In our case, it is more relevant to compare efficiencies to conclude to the role played by silicon quantum dots as optical absorption enhancers. 


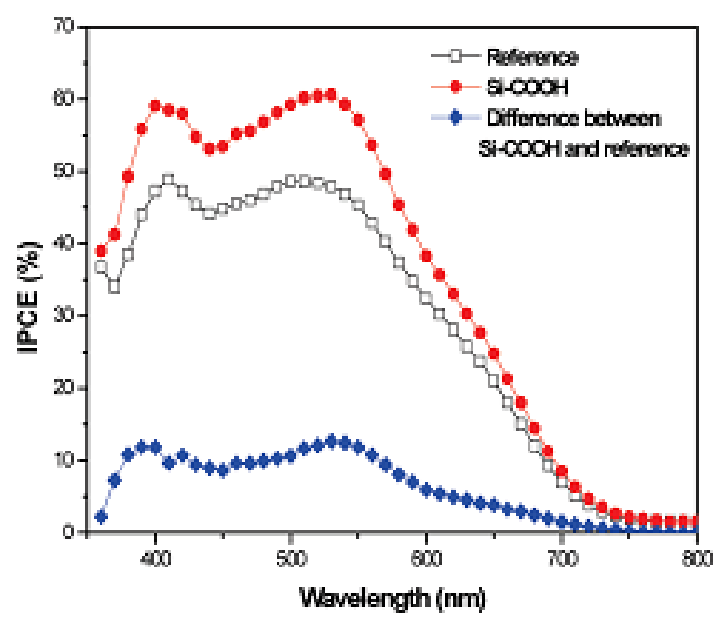

Figure 6.17: IPCE spectra of dye sensitized solar cells treated (solid circles) and untreated (open squares) with the Si nanoparticles and the difference spectrum (solid diamonds) between the two spectra. Reproduction from Kim et al. ${ }^{25}$

The IPCE spectrum obtained by Kim et al (figure 6.17), for their dye-sensitized $\mathrm{TiO}_{2}$ solar cell incorporating silicon nanoparticles show an increased quantum efficiency over the entire range of wavelength of visible light. ${ }^{25}$ This finding is consistent with our theory in this section that silicon quantum dots have mostly a role as light absorption enhancers.

\subsection{Discussion and summary}

\section{SiQDs attached to ZnO nanowires}

The growth of $\mathrm{ZnO}$ nanowires was successful, but it has been found challenging to produce large areas of monodisperse nanowires. ${ }^{37,38}$ The loading of particles by immersion of $\mathrm{ZnO}$ nanowires arrays into a silicon quantum dots solution is a simple method for decorating $\mathrm{ZnO}$ nanowires with SiQDs, ${ }^{11,17}$ but the efficient loading is difficult to control. Spin-coating of concentrated silicon quantum dots solution would be a more reliable method to get rid of the dependence of time and concentration. ${ }^{62} \mathrm{~A}$ surface modification of the $\mathrm{ZnO}$ nanowiress could be envisaged to facilitate the attachment of silicon quantum dots, possibly through hydrogen bonding. ${ }^{25}$ 
The absorption of the composite ZnO NWs/SiQDs show a slight increase over the UV region compared to the neat $\mathrm{ZnO} N W$ s. This increase is located in the same wavelength domain as silicon quantum dots in solution, so it is consistent with the attachment of silicon nanocrystals to the surface of $\mathrm{ZnO}$ nanowires.

The photoluminescence of the composite $\mathrm{ZnO}$ NWs/SiQDs present an increase of the $368 \mathrm{~nm}$ peak ( for $\lambda$ exc=320 nm) upon soaking time into the silicon quantum dots solution, up to 6 hours. As the $368 \mathrm{~nm}$ emission is characteristic for silicon quantum dots (see chapter 3), the increased emission is associated to an augmentation of the concentration of silicon quantum dots anchored to ZnO NWs. After 6 hours, the photoluminescence intensity decreases. A reasonable explanation is that as $\mathrm{ZnO} N W s$ are sensitive to $\mathrm{pH}$, a prolonged bath in a silicon QDs solution, which is slightly acidic, could eventually dissolve ZnO NWs. Therefore, the most appropriate procedure would be soaking for a maximum of 6 hours.

\section{SiQDs blended with block copolymers}

An easy processing without high temperature during formation combined to conserved optical properties of the silicon quantum dots, BCP/SiQDs composite could develop a great interest in optical devices applications.

Watkins et al first reported the controlled structure of block copolymers (BCP) using silicon quantum dots to enhance the separation of the two amphiphillic polymers. ${ }^{13}$ In this research, the experimental protocol used to create films of BCP/SiQDs composite is expected to form a cylindrical geometry.

Using BCP permits an easy loading of the nanoparticles as they are blended in with the copolymer before heating treatment. Films are formed by simply drop casting the polymer solution onto a substrate and the temperature required to enhance the BCP separation is only $100^{\circ} \mathrm{C}$.

Visually, Si QDs still present good optical properties loaded in the block copolymer and the blue luminescence is easily observable under UV light. The increase in light absorption is difficult to evaluate accurately as the PS-PEO block copolymer used in this 
study has an absorption feature similar to silicon quantum dots. Nevertheless, an increase in the absorption edge attests to the contribution of silicon quantum dots to the optical absorption of the composite. No quenching of the photoluminescence of silicon quantum dots is observed while blended in PS-PEO. The PL wavelength of the silicon quantum dots does not vary in the block copolymer composite. As polymers are really big molecules, the interaction with the surface of our silicon quantum dots is limited, therefore no electronic interaction between the two polymers and the silicon quantum dots can be seen in the fluorescence measurements.

\section{SiQDs as an added layer onto a silicon solar cell}

Silicon quantum dots solution were drop cast onto a 'spin-on-dopant' silicon solar cell. Three different silicon nanocrystals samples were used in this study: monodisperse-1hexene, monodisperse-allylamine and polydisperse-allylamine. The contribution brought by silicon quantum dots to the efficiency of the solar cell was assessed by measuring the current density - voltage characteristic. All the silicon quantum dots layers increase the short circuit current density compared to the untreated cell, while the open circuit voltage remains almost constant. The monodisperse 1-henene capped SiQDs coating lead to a significant decreased efficiency and fill factor, resulting from a damage caused to the cell by the chloroform used as a solvent for those silicon nanoparticles. The most effective silicon quantum dots coating was the polydisperse-allylamine SiQDs coating, raising the efficiency of the untreated cell by $9 \%$. Polydisperse nanoparticles seem to create the highest increase in efficiency, most likely due to the uneven SiQDs film surface. Even if the open circuit voltage for all the silicon quantum dots coatings was higher than the one obtained for the commercial anti-reflective coating, the general efficiency of the cell was higher for the cell coated with the titanium silica anti-reflective layer. The general efficiency of the solar cell is increased upon adding a layer of allylamine-terminated silicon nanocrystals. This increase is mostly related to the optical absorption enhancement and the decrease of the reflection of the incident light. In this chapter was demonstrated the influence of silicon quantum dots laid on the surface of a planar silicon solar cell. Further investigation was conducted by Senthuran Sivasubramaniam. Thanks to his expertise in textured surfaces, it was demonstrated that 
the short circuit current enhancement in inverted nanopyramid silicon solar cells combined to an enhanced Jsc by about $20 \%$. The nanoscale texturing combined to a silicon quantum dots layer show a promising approach for the next generation of cheaper and highly efficient solar cells. ${ }^{63}$

\section{Overall conclusion}

All three methods described in this chapter show promising results for the use of silicon quantum dots in photovoltaic devices. To my opinion, the SiQDs blended in block copolymer show the most potential due to the ease of processing and the conservation of the SiQDs optical properties. Even if BCP solar cells show relatively low efficiency so $\mathrm{far}^{52}$ the addition of quantum dots could dramatically improve the photon to electron conversion, in this research is shown that the optical properties of the silicon quantum dots are conserved in a Quantum dot-Block copolymer hybrid composite. The processing can be easily applied to a roll-to-roll fabrication on flexible substrate for flexible transparent devices. 


\subsection{References}

1_Resch-Genger, U.; Grabolle, M.; Cavaliere-Jaricot, S.; Nitschke, R.; Nann, T., Quantum dots versus organic dyes as fluorescent labels. Nat Meth 2008, 5 (9), 763-775.

2_Hod, I.; González-Pedro, V.; Tachan, Z.; Fabregat-Santiago, F.; Mora-Seró, I.; Bisquert, J.; Zaban, A., Dye versus Quantum Dots in Sensitized Solar Cells: Participation of Quantum Dot Absorber in the Recombination Process. The Journal of Physical Chemistry Letters 2011, 2 (24), 3032-3035.

3_ Kamat, P. V., Quantum Dot Solar Cells. The Next Big Thing in Photovoltaics. The Journal of Physical Chemistry Letters 2013, 4 (6), 908-918.

4_ Nozik, A. J., Quantum dot solar cells. Physica E: Low-dimensional Systems and Nanostructures 2002, 14 (1-2), 115-120.

5_ Robel, I.; Subramanian, V.; Kuno, M.; Kamat, P. V., Quantum Dot Solar Cells. Harvesting Light Energy with CdSe Nanocrystals Molecularly Linked to Mesoscopic TiO2 Films. Journal of the American Chemical Society 2006, 128 (7), 2385-2393.

6_ Yu, X.-Y.; Liao, J.-Y.; Qiu, K.-Q.; Kuang, D.-B.; Su, C.-Y., Dynamic Study of Highly Efficient CdS/CdSe Quantum Dot-Sensitized Solar Cells Fabricated by Electrodeposition. ACS Nano 2011, 5 (12), 9494-9500.

7_ Derfus, A. M.; Chan, W. C. W.; Bhatia, S. N., Probing the Cytotoxicity of Semiconductor Quantum Dots. Nano Letters 2003, 4 (1), 11-18.

8_Plank, N. O. V.; Howard, I.; Rao, A.; Wilson, M. W. B.; Ducati, C.; Mane, R. S.; Bendall, J. S.; Louca, R. R. M.; Greenham, N. C.; Miura, H.; Friend, R. H.; Snaith, H. J.; Welland, M. E., Efficient ZnO Nanowire Solid-State Dye-Sensitized Solar Cells Using Organic Dyes and Core-shell Nanostructures. The Journal of Physical Chemistry C 2009, 113 (43), 1851518522. 
9_Divitini, G.; Plank, N. O. V.; Snaith, H. J.; Welland, M. E.; Ducati, C., High-resolution TEM characterization of $\mathrm{ZnO}$ core-shell nanowires for dye-sensitized solar cells. Journal of Physics: Conference Series 2010, 241 (1), 012031.

10_Pradhan, B.; Batabyal, S. K.; Pal, A. J., Vertically aligned ZnO nanowire arrays in Rose Bengal-based dye-sensitized solar cells. Solar Energy Materials and Solar Cells 2007, 91 (9), 769-773.

11_ Pingrong Yu, ¥ Kai Zhu, Andrew G. Norman, Suzanne Ferrere, Arthur J. Frank, and; Nozik, A. J., Nanocrystalline TiO2 Solar Cells Sensitized with InAs Quantum Dots. J. Phys. Chem. 2006, 110, 25451-25454.

12_Lee, Y.-L.; Lo, Y.-S., Highly Efficient Quantum-Dot-Sensitized Solar Cell Based on CoSensitization of CdS/CdSe. Advanced Functional Materials 2009, 19 (4), 604-609.

13_ Lin, Y.; Daga, V. K.; Anderson, E. R.; Gido, S. P.; Watkins, J. J., Nanoparticle-Driven Assembly of Block Copolymers: A Simple Route to Ordered Hybrid Materials. Journal of the American Chemical Society 2011, 133 (17), 6513-6516.

14_Shockley, W.; Queisser, H. J., Detailed Balance Limit of Efficiency of p-n Junction Solar Cells. Journal of Applied Physics 1961, 32 (3), 510-519.

15_http://sharp-world.com/corporate/news/130614.html

16_Yamaguchi, M., Multi-junction solar cells and novel structures for solar cell applications. Physica E: Low-dimensional Systems and Nanostructures 2002, 14 (1-2), 84-90.

17_ Yamaguchi, M.; Takamoto, T.; Araki, K., Super high-efficiency multi-junction and concentrator solar cells. Solar Energy Materials and Solar Cells 2006, 90 (18-19), 30683077. 
18_King, R. R.; Law, D. C.; Edmondson, K. M.; Fetzer, C. M.; Kinsey, G. S.; Yoon, H.; Sherif, R. A.; Karam, N. H., 40\% efficient metamorphic GalnP/GalnAs/Ge multijunction solar cells. Applied Physics Letters 2007, 90 (18), 183516-3.

19_Yu, G.; Gao, J.; Hummelen, J. C.; Wudl, F.; Heeger, A. J., Polymer Photovoltaic Cells: Enhanced Efficiencies via a Network of Internal Donor-Acceptor Heterojunctions. Science 1995, 270 (5243), 1789-1791.

20_Scharber, M. C.; Sariciftci, N. S., Efficiency of bulk-heterojunction organic solar cells. Progress in Polymer Science (0).

21_Pritesh, H.; Husnu Emrah, U.; Gehan, A. J. A., Nanowires for energy generation. Nanotechnology 2012, 23 (19), 194002.

22_Baxter, J. B.; Aydil, E. S., Nanowire-based dye-sensitized solar cells. Applied Physics Letters 2005, 86 (5), 053114-3.

23_Law, M.; Greene, L. E.; Johnson, J. C.; Saykally, R.; Yang, P., Nanowire dye-sensitized solar cells. Nat Mater 2005, 4 (6), 455-459.

24_Greene, L. E.; Law, M.; Tan, D. H.; Montano, M.; Goldberger, J.; Somorjai, G.; Yang, P., General route to vertical $\mathrm{ZnO}$ nanowire arrays using textured $\mathrm{ZnO}$ seeds. Nano Lett 2005, 5 (7), 1231-6.

25_Conibeer, G.; Green, M.; Corkish, R.; Cho, Y.; Cho, E.-C.; Jiang, C.-W.; Fangsuwannarak, T.; Pink, E.; Huang, Y.; Puzzer, T.; Trupke, T.; Richards, B.; Shalav, A.; Lin, K.-I., Silicon nanostructures for third generation photovoltaic solar cells. Thin Solid Films 2006, 511-512 (0), 654-662.

26_Beard, M. C.; Knutsen, K. P.; Yu, P.; Luther, J. M.; Song, Q.; Metzger, W. K.; Ellingson, R. J.; Nozik, A. J., Multiple Exciton Generation in Colloidal Silicon Nanocrystals. Nano Letters 2007, 7 (8), 2506-2512. 
27_Timmerman,D; Izeddin,I; Stallinga,P; Yassievich, I. N.; GregorkiewiczT, Spaceseparated quantum cutting with silicon nanocrystals for photovoltaic applications. Nat Photon 2008, 2 (2), 105-109.

28_Beard, M. C.; Midgett, A. G.; Hanna, M. C.; Luther, J. M.; Hughes, B. K.; Nozik, A. J., Comparing Multiple Exciton Generation in Quantum Dots To Impact Ionization in Bulk Semiconductors: Implications for Enhancement of Solar Energy Conversion. Nano Letters 2010, 10 (8), 3019-3027.

29_Uchida, G.; Sato, M.; Seo, H.; Kamataki, K.; Itagaki, N.; Koga, K.; Shiratani, M., Characteristics of photocurrent generation in the near-ultraviolet region in Si quantumdot sensitized solar cells. Thin Solid Films 2013, 544 (0), 93-98.

30_Conibeer, G.; Green, M.; Cho, E.-C.; König, D.; Cho, Y.-H.; Fangsuwannarak, T.; Scardera, G.; Pink, E.; Huang, Y.; Puzzer, T.; Huang, S.; Song, D.; Flynn, C.; Park, S.; Hao, X.; Mansfield, D., Silicon quantum dot nanostructures for tandem photovoltaic cells. Thin Solid Films 2008, 516 (20), 6748-6756.

31_Huang, S.; Conibeer, G., Sputter-grown Si quantum dot nanostructures for tandem solar cells. Journal of Physics D: Applied Physics 2013, 46 (2), 024003.

32_Liu, C.-Y.; Holman, Z. C.; Kortshagen, U. R., Hybrid Solar Cells from P3HT and Silicon Nanocrystals. Nano Letters 2008, 9 (1), 449-452.

33_Kim, Y.; Kim, C.-H.; Lee, Y.; Kim, K.-J., Enhanced Performance of Dye-Sensitized TiO2

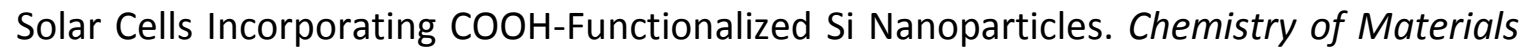
2009, 22 (1), 207-211.

34_Liu, B.; Aydil, E. S., Growth of Oriented Single-Crystalline Rutile TiO2 Nanorods on Transparent Conducting Substrates for Dye-Sensitized Solar Cells. Journal of the American Chemical Society 2009, 131 (11), 3985-3990. 
35_Leschkies, K. S.; Jacobs, A. G.; Norris, D. J.; Aydil, E. S., Nanowire-quantum-dot solar cells and the influence of nanowire length on the charge collection efficiency. Applied Physics Letters 2009, 95 (19), 193103-193103-3.

36_ Vayssieres, L., Growth of Arrayed Nanorods and Nanowires of ZnO from Aqueous Solutions. advanced materials 2003, 15 (5), 464-466.

37_Plank, N. O. V.; et al., A simple low temperature synthesis route for $\mathrm{ZnO}-\mathrm{MgO}$ coreshell nanowires. Nanotechnology 2008, 19 (46), 465603.

38_ Guijarro, N. s.; Lana-Villarreal, T.; Mora-Seró, I. n.; Bisquert, J.; Gómez, R., CdSe Quantum Dot-Sensitized TiO2 Electrodes: Effect of Quantum Dot Coverage and Mode of Attachment. The Journal of Physical Chemistry C 2009, 113 (10), 4208-4214.

39_Leschkies, K. S.; Divakar, R.; Basu, J.; Enache-Pommer, E.; Boercker, J. E.; Carter, C. B.; Kortshagen, U. R.; Norris, D. J.; Aydil, E. S., Photosensitization of ZnO Nanowires with CdSe Quantum Dots for Photovoltaic Devices. Nano Letters 2007, 7 (6), 1793-1798.

40_Zaban, A.; Mićić, O. I.; Gregg, B. A.; Nozik, A. J., Photosensitization of Nanoporous TiO2 Electrodes with InP Quantum Dots. Langmuir 1998, 14 (12), 3153-3156..

41_Warner, J. H.; Hoshino, A.; Yamamoto, K.; Tilley, R. D., Water-Soluble Photoluminescent Silicon Quantum Dots. Angewandte Chemie International Edition 2005, 44 (29), 4550-4554.

42_ Zhou, Z.; Brus, L.; Friesner, R., Electronic Structure and Luminescence of 1.1- and 1.4-nm Silicon Nanocrystals: Oxide Shell versus Hydrogen Passivation. Nano Letters 2003, 3 (2), 163-167.

43_Greenwood, Norman N.; Earnshaw, Alan (1997). Chemistry of the Elements (2nd ed.). Butterworth-Heinemann 
44_Spero, J. M.; Devito, B.; Theodore, L. (2000). Regulatory chemical handbook. CRC Press.

45_Chang, W.-C.; Lee, C.-H.; Yu, W.-C.; Lin, C.-M., Optimization of dye adsorption time and film thickness for efficient $\mathrm{ZnO}$ dye-sensitized solar cells with high at-rest stability. Nanoscale Research Letters 2012, 7 (1), 1-10.

46_Brabec, C. J.; Sariciftci, N. S.; Hummelen, J. C., Plastic Solar Cells. Advanced Functional Materials 2001, 11 (1), 15-26.

47_Westenhoff, S.; Howard, I. A.; Hodgkiss, J. M.; Kirov, K. R.; Bronstein, H. A.; Williams, C. K.; Greenham, N. C.; Friend, R. H., Charge Recombination in Organic Photovoltaic Devices with High Open-Circuit Voltages. Journal of the American Chemical Society 2008, 130 (41), 13653-13658.

48_http://optics.org/news/4/1/36

49_Heo, J. H.; Im, S. H.; Noh, J. H.; Mandal, T. N.; Lim, C.-S.; Chang, J. A.; Lee, Y. H.; Kim, H.-j.; Sarkar, A.; NazeeruddinMd, K.; Gratzel, M.; Seok, S. I., Efficient inorganic-organic hybrid heterojunction solar cells containing perovskite compound and polymeric hole conductors. Nat Photon 2013, 7 (6), 486-491.

50_Ren, S.; Chang, L.-Y.; Lim, S.-K.; Zhao, J.; Smith, M.; Zhao, N.; Bulović, V.; Bawendi, M.; Gradečak, S., Inorganic-Organic Hybrid Solar Cell: Bridging Quantum Dots to Conjugated Polymer Nanowires. Nano Letters 2011, 11 (9), 3998-4002.

51_Sun, S.-S.; Zhang, C.; Ledbetter, A.; Choi, S.; Seo, K.; Bonner, C. E.; Drees, M.; Sariciftci, N. S., Photovoltaic enhancement of organic solar cells by a bridged donoracceptor block copolymer approach. Applied Physics Letters 2007, 90 (4), 043117043117-3. 
52_. Guo, C.; Lin, Y.-H.; Witman, M. D.; Smith, K. A.; Wang, C.; Hexemer, A.; Strzalka, J.; Gomez, E. D.; Verduzco, R., Conjugated Block Copolymer Photovoltaics with near 3\% Efficiency through Microphase Separation. Nano Letters 2013, 13 (6), 2957-2963.

53_Alexandridis P, Lindman B. (2000) "Amphiphilic block copolymers: self assembly and applications". Amsterdam: Elsevier

54_Mattoussi, H.; Radzilowski, L. H.; Dabbousi, B. O.; Fogg, D. E.; Schrock, R. R.; Thomas, E. L.; Rubner, M. F.; Bawendi, M. G., Composite thin films of CdSe nanocrystals and a surface passivating/electron transporting block copolymer: Correlations between film microstructure by transmission electron microscopy and electroluminescence. Journal of Applied Physics 1999, 86 (8), 4390-4399.

55_Zorn, M.; Bae, W. K.; Kwak, J.; Lee, H.; Lee, C.; Zentel, R.; Char, K., Quantum Dot-Block Copolymer Hybrids with Improved Properties and Their Application to Quantum Dot Light-Emitting Devices. ACS Nano 2009, 3 (5), 1063-1068.

56_Wenham, S. R.; Green, M. A., Silicon solar cells. Progress in Photovoltaics: Research and Applications 1996, 4 (1), 3-33.

57_Fitting Kourkoutis, L.; Hao, X.; Huang, S.; Puthen-Veettil, B.; Conibeer, G.; Green, M.; Perez-Wurfl, I., Three-dimensional imaging for precise structural control of Si quantum dot networks for all-Si solar cells. Nanoscale 2013.

58_Yi-Yu, L., Wen-Jeng, H., Jhih-Kai, S., Quan-Ru, L; Cheng-Ming, Y, 17.9\% Efficiency Silicon Solar Cells by Using Spin-on Films Processes PIERS Proceedings, 2011

59_Senthuran Sivasubramaniam personnal design [senthuran.sivasubramaniam@pg.canterbury.ac.nz]

60_http://www.emulsitone.com/tisif.html 
61_http://abet-technologies.com/solar-simulators/sun-3000-class-aaa

62_Jean, J.; Chang, S.; Brown, P. R.; Cheng, J. J.; Rekemeyer, P. H.; Bawendi, M. G.; Gradečak, S.; Bulović, V., ZnO Nanowire Arrays for Enhanced Photocurrent in PbS Quantum Dot Solar Cells. advanced materials 2013, 25 (20), 2790-2796.

63_ Sivasubramaniam, S.; Faramus, A.; Tilley, R. D.; Alkaisi, M. M., Performance enhancement in silicon solar cell by inverted nanopyramid texturing and silicon quantum dots coating. Journal of Renewable and Sustainable Energy 2014, 6 (1), - 



\section{Conclusion}

Quantum dot solar cells have considerable potential as a new generation of photovoltaic devices. From the results in this thesis, some overarching conclusions about semiconductor nanoparticles can be made in this context. This thesis focuses on the synthesis and the characterization of semiconductor nanocrystals with the possibility of using them as photosensitizers in solar cells. Four types of nanoparticles were investigated: silicon and germanium quantum dots, CZTS nanocrystals and finally tin and tin/germanium alloy nanoparticles.

\subsection{Micro-emulsion synthesis and effect of reducing agent}

The synthesis via chemical reduction in reverse micelles at room temperature seems to produce smaller particles than high temperature decomposition methods, regardless of the materials used.

In chemical reduction using a micro-emulsion synthetic process, the hydride reducing agent plays an important role in the control of the growth of the nanoparticles. Using different strength of reducing agents leads to a variation of the size distribution of the nanocrystals. For silicon nanocrystals, the size of the nanocrystals is decreased from 3.7 $\mathrm{nm}$ to $2.3 \mathrm{~nm}$ and further to $1.5 \mathrm{~nm}$ by switching from sodium borohydride to lithium borohydride to lithium aluminium hydride as reducing agents, respectively. This size variation is in good agreement with the results of Prabakar et al for germanium quantum dots and Shiohara et al for silicon quantum dots, respectively. ${ }^{1,2}$

In the case of CZTS nanocrystals, the chemical reduction led to the production of multiple phases instead of pure CZTS phase due to a difference of reactivity of the four precursors. Nevertheless, the particles presented a much smaller size than CZTS nanocrystals synthesized by high temperature decomposition. $\mathrm{SnS}, \mathrm{ZnS}$ and $\mathrm{Cu}_{2} \mathrm{~S}$ were synthesized via the same chemical reduction process, which, so far, has never been reported for those materials. All three metal sulphides synthesized via microemulsion presented a small size distribution. 
The chemical reduction of germanium tetrachloride using hydride reducing agents yields smaller particles than other synthetic techniques reported in the literature. ${ }^{3,4}$ Also, the reverse micelle technique allows a better size control and consequently a much narrower size distribution than other solution-phase techniques. To conserve the optical properties of germanium quantum dots, a good size control is essential as the bulk germanium form does not present any fluorescence.

Finally, metallic tin nanoparticles have been synthesized via the micro-emulsion technique and were found to be $4.3 \mathrm{~nm}$ on average, which is much smaller than tin nanoparticles obtained by reported methods, when synthesized at room temperature. ${ }^{5,6}$ The chemical reduction of tin nanoparticles in a solvent media cooled down to $0^{\circ} \mathrm{C}$ produced a much larger size than the tin nanoparticles produced via the same process at room temperature. At room temperature, using a strong reducing agent creates a "burst" nucleation, meaning that monomers are created almost simultaneously and thus, according to the LaMer model, it results in a better size control and a small size distribution. ${ }^{7}$ At $0^{\circ} \mathrm{C}$, the reducing agent takes longer to react, leading to a longer nucleation process and by all means a larger size distribution. At high temperature, reducing agents provoke violent reactions as described in chapter 5 for CZTS nanocrystals and are to be avoided.

\subsection{Optical properties and surface effects}

Silicon and germanium quantum dots are excellent luminescent emitters. Germanium nanocrystals exhibit a luminescent quantum yield as high as $16 \%$. Also, the high ratio between surface area and volume for nanoparticles tends to enhance the surface effects on the UV-Visible absorption and the fluorescence.

In the case of silicon quantum dots, as the size distribution decreases, the UV-Visible absorption edge wavelength gets longer. The blue shift of the absorption edge is consistent with the direct band gap theory and the increase of band gap energy value can be associated to quantum confinement or a statistically reduced localization possibility of electrons and holes due to the size proximity to the Bohr radius. Also, in chapter 3 is studied the influence on the optical properties of silicon quantum dots 
capped with three different organic ligands. No absorption or emission ligand dependence was evidenced, leading to the conclusion than the optical properties of silicon quantum dots is not affected by the functional group of the ligand molecule. Nevertheless, the fluorescence originates in both the direct band gap transition due to quantum confinement and an electron transfer between the silicon surface atoms and their immediate carbon neighbours via a covalent bond.

Interestingly, a similar experiment performed on germanium quantum dots showed that the emission is a complex phenomenon, driven by at least four different emission processes. It is highly influenced by the surface termination, mostly in photoluminescence intensity at a given wavelength. The multi transition emission process cannot be explained by the direct band gap theory alone and surface trap states are probably also at the origin of the photoluminescence of germanium quantum dots.

\subsection{Band gap properties for solar cell applications}

Shockley Queisser determined that the optimum bandgap for the light absorbers in a single junction solar cell is $1.4 \mathrm{eV}^{8}{ }^{8}$ With a bulk band gap of $1.4-1.6 \mathrm{eV}, \mathrm{CZTS}$ is an ideal candidate. Using high temperature decomposition techniques, the CZTS nanocrystals were found to present a bulk band gap. ${ }^{9,10}$ Khare et al in 2011 reported the only case of quantum confined CZTS nanocrystals. ${ }^{11}$ Microemulsion synthesis was expected to bring a better size control of the CZTS nanocrystals down to a quantum confined state with a band gap increase with a decrease of diameter. Further work will be required to achieve a pure CZTS phase through this room temperature process.

In its semiconducting form, bulk tin has a small bandgap of $0.08 \mathrm{eV}$. There is a great potential for quantum confined $\alpha$-tin to present a bandgap in the optimum range for solar light absorption with quantum confinement. However, the synthesis of $\alpha$-tin in nanoparticle spatial confinement proved very difficult and has not been achieved yet via a solution process due probably to the $\alpha$-tin phase instability under ambient conditions.

Silicon quantum dots synthesized via the microemulsion process present a bandgap energy of $3.4 \mathrm{eV}$, which is significantly larger than the $1.1 \mathrm{eV}$ band gap of bulk silicon. 
This increased bandgap is one of the explanations for the bright blue luminescence observed for silicon quantum dots along surface effects. A similar trend is observed for germanium quantum dots, which band gap increases up to $3.5 \mathrm{eV}$ in the quantum confinement when synthesized via the microemulsion process. As germanium has a bandgap of $0.67 \mathrm{eV}$ in its bulk form, the increase of energy gap can explain the fluorescence observed for those nanocrystals.

\subsection{Photovoltaic applications}

Silicon and germanium quantum dots are not commonly found in QDSSCs research. Si and Ge QDs have band gaps too high for the optimum light absorption in a single junction solar cell. Nevertheless, they attracted attention as "hot carrier" absorbers, collecting photons with high energies and limiting the solar cells losses due to thermalization. ${ }^{12-14}$ In this research (chapter 4), we report the use of silicon quantum dots used in solar cells. Using silicon quantum dots as an added layer on top of a doped silicon solar cell generates an increase the overall efficiency by up to $9 \%$, most likely due to an optical absorption enhancement and the decrease of the reflection of the incident sunlight.

As they have complete compatibility with flexible plastic substrate, ZnO nanowires and block copolymers are two different approaches to the construction of solar cells that could easily be applied to quantum dots solar cells systems. ${ }^{15-17}$ With silicon quantum dots, a ZnO nanowire platform suffers from a lack of control of the loading of the nanoparticles into the structure. Block copolymers can easily be blended with quantum dots prior to the heat treatment that give them the desired architecture. Also, when using silicon quantum dots, the quantum dots / block copolymer composite preserves the full optical properties of the colloidal quantum dots.

\subsection{Future work and overall conclusion}

Microemulsion synthesis has proven efficient to produce size controlled nanoparticles of single or binary semiconductors. Ternary and quaternary particles have not yet 
successfully been synthesized via this technique due to a variation of decomposition times between the various precursors. A solution could be found in the research towards either finding the right precursors to reduce the reduction time discrepancy between precursors, or a single precursor source containing the three or four desired elements that would produce simultaneously the three or four different monomers. This approach could remedy the multi phase formation that has been observed in the research with CZTS.

The microemulsion synthesis of silicon quantum dots is hard to scale up and at this point it is difficult to envisage large scale industrial production. Veinot's synthesis technique using the thermolysis of hydrogen silsesquioxane seems a better candidate for large yields of silicon quantum dots. ${ }^{18}$ The downside of this technique is the etching step, using hydrofluoric acid, a highly hazardous chemical.

The origin of the surface effects on the optical properties of silicon and germanium quantum dots could be identified using more advanced spectroscopy techniques, such as ultrafast laser spectroscopy, to pinpoint the origin of each type of electron transition occurring in such small nanocrystals. ${ }^{19}$

Silicon and germanium alloy in a nanocrystal form is of significant interest. Alloying both elements is expected to combine both optical properties. Gradually varying the composition of the binary alloy system $\mathrm{Si}_{x} \mathrm{Ge}_{1-\mathrm{x}}$ will provide a series of nanoparticles with a continuous series of electrical and optical properties. So far, SiGe alloy nanoparticles have been synthesized only via physical synthetic processes. ${ }^{20,21}$ The reverse micelle synthesis of SiGe alloy nanocrystals would be an advancement towards optically tunable and environmentally friendly quantum dots.

The highest recorded efficiency for a photovoltaic device is so far $44.7 \% .^{22}$ This high performance has been obtained for a multi junction solar cell, combining several III-V semiconducting materials with various bandgap to optimize the light absorption range. The same principle can be applied to quantum dots solar cells. Silicon and germanium quantum dots can be combined to CZTS nanocrystals or metal sulphide nanoparticles in a multi layer architecture to absorb most of the wavelengths of sunlight. 
Inorganic blue emitters with high efficiencies are rare and difficult to produce due to the intrinsically wide band gaps and they are a prerequisite for applications as lighting sources and flat-panel displays. ${ }^{23,24}$ The bright blue fluorescence of silicon and germanium quantum dots can be utilized in optoelectronic devices, such as light emitting diodes. The high quantum yield of $16 \%$ for germanium nanocrystals combined to a great resistance to photobleaching makes germanium quantum dots a better emitter material than organic dyes.

The microemulsion synthesis in reverse micelle can be applied to a variety of semiconducting or metallic materials, mostly for primary or binary materials. It presents promises for other interesting materials for solar cells, such as ternary or quaternary nanocrystals. Silicon and germanium quantum dots are efficient blue light emitters and even though the origin of their optical properties is still not completely understood, they have potential for applications in optoelectronic devices. 


\section{7. References}

1_Prabakar, S.; Shiohara, A.; Hanada, S.; Fujioka, K.; Yamamoto, K.; Tilley, R. D., Size Controlled Synthesis of Germanium Nanocrystals by Hydride Reducing Agents and Their Biological Applications. Chemistry of Materials 2009, 22 (2), 482-486.

2_Shiohara, A.; Prabakar, S.; Faramus, A.; Hsu, C.-Y.; Lai, P.-S.; Northcote, P. T.; Tilley, R. D., Sized controlled synthesis, purification, and cell studies with silicon quantum dots. Nanoscale 2011, 3 (8), 3364-3370.

3_Vaughn li, D. D.; Schaak, R. E., Synthesis, properties and applications of colloidal germanium and germanium-based nanomaterials. Chemical Society Reviews 2013, 42 (7), 2861-2879.

4_Stoldt, C. R.; Haag, M. A.; Larsen, B. A., Preparation of freestanding germanium nanocrystals by ultrasonic aerosol pyrolysis. Applied Physics Letters 2008, 93 (4), 043125-3.

5_Li, Z.; Tao, X.; Cheng, Y.; Wu, Z.; Zhang, Z.; Dang, H., A facile way for preparing tin nanoparticles from bulk tin via ultrasound dispersion. Ultrasonics Sonochemistry 2007, $14(1), 89-92$.

6_Jo, Y. H.; et al., Synthesis and characterization of low temperature Sn nanoparticles for the fabrication of highly conductive ink. Nanotechnology 2011, 22 (22), 225701.

7_ LaMer, V. K.; Dinegar, R. H., Theory, Production and Mechanism of Formation of Monodispersed Hydrosols. Journal of the American Chemical Society 1950, 72 (11), 4847-4854.

8_Shockley, W.; Queisser, H. J., Detailed Balance Limit of Efficiency of p-n Junction Solar Cells. Journal of Applied Physics 1961, 32 (3), 510-519. 
9_ Steinhagen, C.; Panthani, M. G.; Akhavan, V.; Goodfellow, B.; Koo, B.; Korgel, B. A., Synthesis of Cu2ZnSnS4 Nanocrystals for Use in Low-Cost Photovoltaics. Journal of the American Chemical Society 2009, 131 (35), 12554-12555.

10_Riha, S. C.; Parkinson, B. A.; Prieto, A. L., Solution-Based Synthesis and Characterization of Cu2ZnSnS4 Nanocrystals. Journal of the American Chemical Society 2009, 131 (34), 12054-12055.

11_Khare, A.; Wills, A. W.; Ammerman, L. M.; Norris, D. J.; Aydil, E. S., Size control and quantum confinement in Cu2ZnSnS4 nanocrystals. Chemical Communications 2011, 47 (42), 11721-11723.

12_Conibeer, G. J.; Jiang, C. W.; König, D.; Shrestha, S.; Walsh, T.; Green, M. A., Selective energy contacts for hot carrier solar cells. Thin Solid Films 2008, 516 (20), 6968-6973.

13_Tayagaki, T.; Usami, N.; Pan, W.; Hoshi, Y.; Ooi, K.; Kanemitsu, Y., Enhanced carrier extraction from Ge quantum dots in Si solar cells under strong photoexcitation. Applied Physics Letters 2012, 101 (13), 133905-4.

14_Nozik, A. J.; Beard, M. C.; Luther, J. M.; Law, M.; Ellingson, R. J.; Johnson, J. C., Semiconductor Quantum Dots and Quantum Dot Arrays and Applications of Multiple Exciton Generation to Third-Generation Photovoltaic Solar Cells. Chemical Reviews 2010, $110(11), 6873-6890$.

15_Jean, J.; Chang, S.; Brown, P. R.; Cheng, J. J.; Rekemeyer, P. H.; Bawendi, M. G.; Gradečak, S.; Bulović, V., ZnO Nanowire Arrays for Enhanced Photocurrent in PbS Quantum Dot Solar Cells. advanced materials 2013, 25 (20), 2790-2796.

16_Boucharef, M.; Di Bin, C.; Boumaza, M. S.; Colas, M.; Snaith, H. J.; Ratier, B.; Boucle, J., Solid-state dye-sensitized solar cells based on ZnO nanocrystals. Nanotechnology 2010, 21 (20), 205203 (12 pp.)-205203 (12 pp.). 
17_Lin, Y.; Daga, V. K.; Anderson, E. R.; Gido, S. P.; Watkins, J. J., Nanoparticle-Driven Assembly of Block Copolymers: A Simple Route to Ordered Hybrid Materials. Journal of the American Chemical Society 2011, 133 (17), 6513-6516.

18_ Hessel, C. M.; Henderson, E. J.; Veinot, J. G. C., Hydrogen Silsesquioxane: A Molecular Precursor for Nanocrystalline Si-SiO2 Composites and Freestanding HydrideSurface-Terminated Silicon Nanoparticles. Chemistry of Materials 2006, 18 (26), 61396146.

19_Chiu, C.-C.; Hung, C.-C.; Chen, C.-L.; Cheng, P.-Y., Ultrafast Time-Resolved Broadband Fluorescence Studies of the Benzene-Tetracyanoethylene Complex: Solvation, Vibrational Relaxation, and Charge Recombination Dynamics. The Journal of Physical Chemistry B 2013, 117 (33), 9734-9756.

20_ Yasar-Inceoglu, O.; Mangolini, L., Characterization of Si-Ge alloy nanocrystals produced in a non-thermal plasma reactor. Materials Letters 2013, 101 (0), 76-79.

21_Derin, H.; Kantarlı, K.; Yıldız, M.; Dost, S., Optical properties of SixGe1-x single crystals grown by liquid phase diffusion. Materials Science in Semiconductor Processing 2009, $12(4-5), 146-150$.

22_http://phys.org/news/2013-09-world-solar-cell-efficiency.html

23_Zhu, M.; Yang, C., Blue fluorescent emitters: design tactics and applications in organic light-emitting diodes. Chemical Society Reviews 2013, 42 (12), 4963-4976.

24_Oner, I., Stathatos, E.; Varlikli, C.; White Light Electroluminescence by OrganicInorganic Heterostructures with CdSe Quantum Dots as Red Light Emitters. Advances in Optical Technologies 2011, 2011 



\section{Appendix 1}

Comparison of the UV-Visible absorption spectrum of tryptophan to the absorption of germanium quantum dots with four different capping

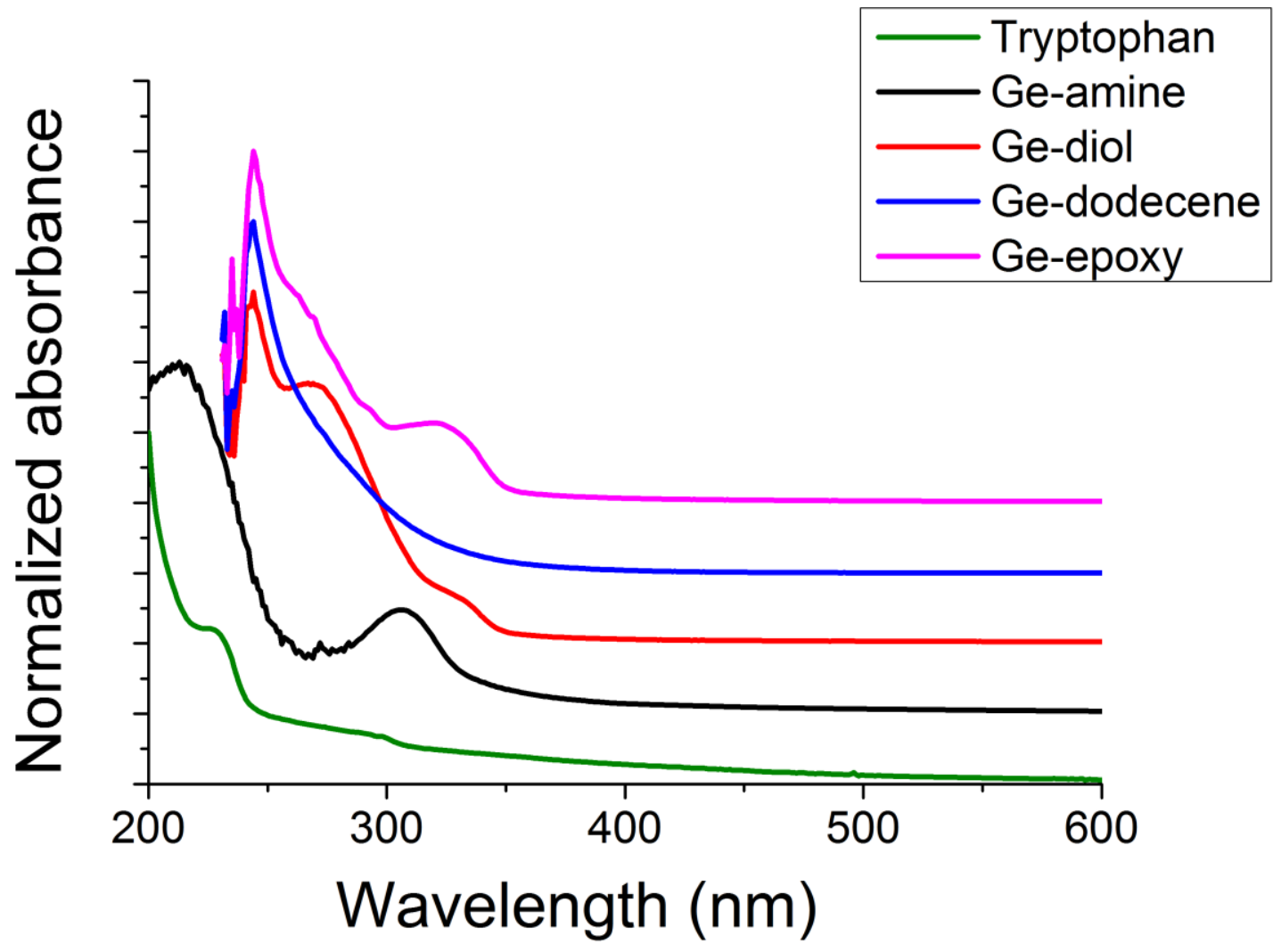





\section{List of publications}

1_Shiohara, A.; Prabakar, S.; Faramus, A.; Hsu, C.-Y.; Lai, P.-S.; Northcote, P. T.; Tilley, R. D., Sized controlled synthesis, purification, and cell studies with silicon quantum dots. Nanoscale 2011, 3 (8), 3364-3370.

2_Dasog, M.; Yang, Z.; Regli, S.; Atkins, T. M.; Faramus, A.; Singh, M. P.; Muthuswamy, E.; Kauzlarich, S. M.; Tilley, R. D.; Veinot, J. G. C., Chemical Insight into the Origin of Red and Blue Photoluminescence Arising from Freestanding Silicon Nanocrystals. ACS Nano 2013, 7 (3), 2676-2685.

3_ Sivasubramaniam, S.; Faramus, A.; Tilley, R. D.; Alkaisi, M. M., Performance enhancement in silicon solar cell by inverted nanopyramid texturing and silicon quantum dots coating. Journal of Renewable and Sustainable Energy 2014, 6 (1), -. 
